\title{
A Study of $B$ Meson Decays Involving a $J / \psi$ Meson
}

by

George Serafim Sganos

A thesis submitted in conformity with the requirements for the degree of Doctor of Philosophy

Graduate Department of Physics

University of Toronto

(C) Copyright by George Serafim Sganos (1996) 
A Study of $B$ Meson Decays Involving a $J / \psi$ Meson

George Serafim Sganos

Doctor of Philosophy, 1996

Graduate Department of Physics

University of Toronto

\begin{abstract}
Three flavors of bottom mesons are observed with the use of the Collider Detector at Fermilab (CDF). The bottom mesons are observed in five fully reconstructed channels containing a $J / \psi$ meson. The five channels (including the charge-conjugate modes) are $B^{+} \rightarrow J / \psi K^{+}, B^{+} \rightarrow J / \psi K^{*}(892)^{+}, B^{0} \rightarrow J / \psi K^{0}, B^{0} \rightarrow J / \psi K^{*}(892)^{0}$ and $B_{s}^{0} \rightarrow J / \psi \phi(1020)$. The $J / \psi$ mesons are observed in the $\mu^{+} \mu^{-}$channel.

The decay rate for each channel is related to the rates of the other decays through a ratio of branching fractions times fragmentation fractions of the $b$ quark into the specific bottom mesons. The measurements of the ratios of branching fractions times fragmentation fractions are presented as a five-by-five matrix.

The ratios of branching fractions are extracted with certain assumptions for the ratios of fragmentation fractions. Branching fractions are then calculated by using world average branching fraction values for the other decays.

Comparisons of the ratios of branching fractions for the $B^{+}$and $B^{0}$ mesons with various phenomenological models show good agreement. The ratios of fragmentation fractions are then calculated using a phenomenological model for the ratios of branching fractions for the bottom mesons. With an additional assumption about the fraction of $b$ quarks that hadronize into $b$ baryons, the fragmentation fractions for $B^{+}, B^{0}$ and $B_{s}^{0}$ mesons are also determined to be
\end{abstract}

$$
\begin{aligned}
& f_{u}=0.39 \pm 0.04(\text { stat }) \pm 0.04(\text { syst }) \\
& f_{d}=0.38 \pm 0.04(\text { stat }) \pm 0.04(\text { syst }) \\
& f_{s}=0.13 \pm 0.04(\text { stat }) \pm 0.01(\text { syst })
\end{aligned}
$$




\section{Résumé}

Trois genres de bottom mesons sont observés avec le Collider Detector au Fermilab (CDF). Les bottom mesons sont observés dans cinq channels completement reconstruits et contenant un $J / \psi$ meson. Les cinq channels (incluant les channels chargés-conjugués) sont $B^{+} \rightarrow J / \psi K^{+}, B^{+} \rightarrow J / \psi K^{*}(892)^{+}, B^{0} \rightarrow J / \psi K^{0}$, $B^{0} \rightarrow J / \psi K^{*}(892)^{0}$ et $B_{s}^{0} \rightarrow J / \psi \phi(1020)$. Les $J / \psi$ mesons sont observés dans le channel $\mu^{+} \mu^{-}$.

Le taux de decay pour chaque channel est relié aux autres decays par un rapport de branching fractions fois les fractions de fragmentation de $b$ quark dans le bottom meson en particulier. Les mesures des rapports de branching fractions fois les fractions de fragmentation sont presentées sous forme d'une matrice cinq par cinq.

Les rapports de branching fractions sont obtenu avec quelques suppositions pour les rapports de fractions de fragmentation. Les branching fractions sont calculées en utilisant la moyenne mondiale des valeurs de branching fraction pour les autres decays.

La comparision des rapport de branching fractions pour le $B^{+}$et $B^{0}$ mesons avec quelques models phenomenologiques montre un bon accord. En utilisant un model phenomenologique pour les rapports de branching fractions pour les bottom mesons, les rapports de fraction de fragmentation sont aussi calculées. En ajoutant une supposition pour la fraction de $b$ quarks que sont hadronisées dans $b$ baryons, les fractions de fragmentation pour les $B^{+}, B^{0}$ et $B_{s}^{0}$ mesons sont déterminés d'être

$$
\begin{aligned}
& f_{u}=0.39 \pm 0.04 \text { (stat) } \pm 0.04 \text { (syst) } \\
& f_{d}=0.38 \pm 0.04 \text { (stat) } \pm 0.04 \text { (syst) } \\
& f_{s}=0.13 \pm 0.03 \text { (stat) } \pm 0.01 \text { (syst). }
\end{aligned}
$$




\section{Acknowledgments}

The results obtained with the CDF detector would not have been possible without the hard work of many people. It is to all these people who were involved in the design, construction and operation of the CDF detector that I would first like to thank. In particular, I would like to thank the members of the IPP group with which I have worked with during the last five years. Special thanks goes to my supervisor Pekka Sinervo for his enthusiasm, his patience and his continuous support. Other members that I would like to acknowledge for their support are Ken Ragan, Kurt Biery, Andreas Hölscher, Klaus Strahl, Wendy Taylor and Bjoern Hinrichsen. I would also like to thank Kostas Kordas for making many of my trips to Fermilab and Chicago worthwhile. I especially appreciated our many Greek discussions. Last but not least, I would like to thank my officemates Hyunsoo Kim and Andreas Warburton not only for the many fruitful discussions on a wide range of topics including fish, strawberries and watermelons as well as the many errands taken care of over the years, but also for helping me keep my sanity through the difficult times.

Financial support received from various levels of government, the University of Toronto and the department of physics is gratefully acknowledged.

I would also like thank my fellow graduate students for making may stay at

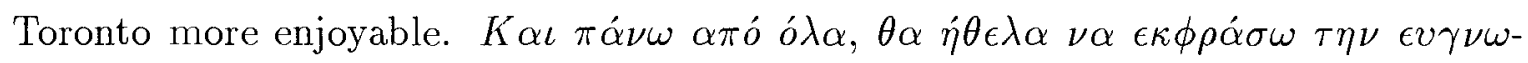

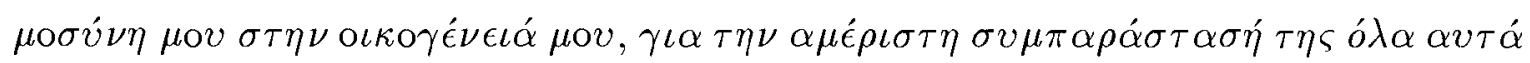
$\tau \alpha \chi \rho o ́ v \iota \alpha$. 


\section{Contents}

$\begin{array}{lll}\text { Abstract } & \text { ii }\end{array}$

Résumé

Acknowledgments

1 Introduction $\quad 1$

1.1 Historical Overview . . . . . . . . . . . . . . . 2

1.2 The Standard Model . . . . . . . . . . . . . . . . . 2

1.3 Production of Bottom Quarks . . . . . . . . . . . . . 5

1.4 Hadronization of Bottom Quarks and Decay of Bottom Hadrons . . . 11

1.5 Non-Leptonic decays of Bottom Mesons . . . . . . . . . . . . . 17

2 The Experimental Apparatus $\quad \mathbf{2 2}$

2.1 The Tevatron Accelerator . . . . . . . . . . . . . . . . 22

2.2 The Collider Detector at Fermilab . . . . . . . . . . . . . 25

2.3 The CDF Trigger System . . . . . . . . . . . . . . . . . 34

2.3 .1 Level 1. . . . . . . . . . . . . . . . . . 35

2.3 .2 Level $2 \ldots \ldots \ldots \ldots \ldots$

$2.3 .3 \quad$ Level $3 \ldots \ldots \ldots \ldots \ldots \ldots$

3 Event Selection $\quad 39$

3.1 Track Quality Requirements . . . . . . . . . . . . . . . . . 40

3.2 Muon and $J / \psi$ Requirements . . . . . . . . . . . . . . 43

3.3 Strange Meson Requirements . . . . . . . . . . . . . 50

3.4 Bottom Meson Requirements . . . . . . . . . . . . . 51

3.5 Primary Vertex Considerations . . . . . . . . . . . . 61 
3.6 Reconstruction of Bottom Mesons . . . . . . . . . . . . . . . 62

3.6.1 Number of Observed Decays . . . . . . . . . . . . . . 63

4 Calculation of Efficiencies $\quad 75$

4.1 Kinematic and Geometric Efficiencies . . . . . . . . . . . . . 75

4.2 Proper Decay Length Cut Efficiencies . . . . . . . . . . . . . . . 81

4.3 CTC Pattern Finding Efficiencies . . . . . . . . . . . . . . . 84

4.4 Confidence Level Requirement Efficiencies . . . . . . . . . . . . 87

5 Discussion of Systematic Uncertainties $\quad 91$

5.1 Muon and $J / \psi$ Requirements . . . . . . . . . . . . . . 91

5.2 Proper Decay Length Requirements . . . . . . . . . . . . . . . 92

5.3 Helicity Distribution . . . . . . . . . . . . . . . . . 93

5.4 Confidence Level Requirements . . . . . . . . . . . . . . . 93

5.5 Charged Kaon Decay in Flight. . . . . . . . . . . . . . . . . 94

5.6 Differential Bottom Quark Production Cross Section . . . . . . . 95

5.6 .1 Shape Effects . . . . . . . . . . . . . . 95

5.6 .2 Effects of Higher Spin States . . . . . . . . . . . 95

6 Ratios of Branching Fractions Times Ratios of $b$ Quark Fragmenta$\begin{array}{ll}\text { tion Fractions } & 98\end{array}$

6.1 Calculation of Matrix Elements . . . . . . . . . . . . . . . . 98

6.2 Calculation of Branching Fractions . . . . . . . . . . . 105

6.3 Calculation of Ratios of Fragmentation Fractions . . . . . . . . . 107

6.4 Estimate of Fragmentation Fractions . . . . . . . . . . . . . 110

7 Summary of Results and Conclusions $\quad 114$

7.1 Measurements of Branching Fractions . . . . . . . . . . . . 115

7.2 Ratios of Fragmentation Fractions . . . . . . . . . . . . 116

7.3 Future Prospects . . . . . . . . . . . . . . . . . . . 118

$\begin{array}{ll}\text { A Effect of Various Vertex Considerations } & 119\end{array}$

B Matrix Elements of Ratio of Events and Ratios of Detection Effi$\begin{array}{ll}\text { ciencies } & 128\end{array}$

$\begin{array}{lr}\text { C The CDF Collaboration } & 147\end{array}$ 


\section{List of Figures}

1.1 The lowest order Feynman diagrams for quark production in hadron

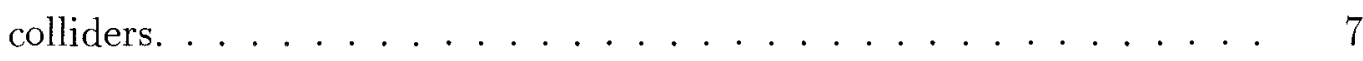

1.2 Some Feynman diagrams that contribute in order of $\alpha_{s}^{3}$ in the parton

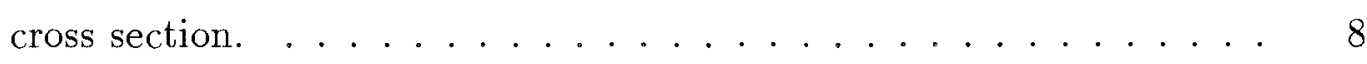

1.3 The $b$ quark production cross section from $\bar{p} p$ collisions at a center-ofmass energy of $1.8 \mathrm{TeV} \ldots \ldots \ldots \ldots \ldots$

1.4 The lowest order Feynman diagram for a two body $B$ meson decay into states involving a $J / \psi$ meson. . . . . . . . . . . . . .

2.1 Schematic of the Linac, Booster, Main Ring, Debuncher/Accumulator

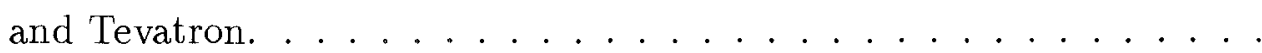

2.2 Schematic of the Linac, Booster, Main Ring, Debuncher, Accumulator

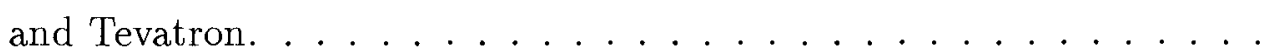

2.3 Isometric display of the CDF detector with a cut-a-way view of one

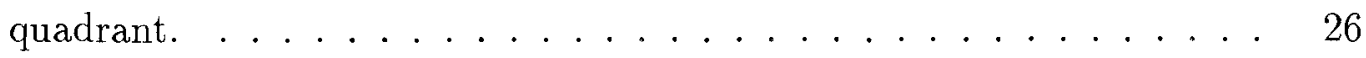

2.4 Longitudinal profile of one quadrant of the CDF detector. . . . . . 27

2.5 Beam line view of the central tracking chamber. . . . . . . . . . 28

2.6 Event display of a candidate $B_{s}^{0}$ decay. . . . . . . . . . . . . . 29

2.7 Layout of the central muon chambers within a central calorimeter wedge. 31

2.8 Layout of the central muon drift cells within a central muon chamber. 33

3.1 Decay chain of the reconstructed $B^{+}$meson into a $J / \psi K^{*}(892)^{+}$pair. 40

3.2 Invariant mass distribution of $\mu^{+} \mu^{-}$pairs before and after the muon segment CTC matching requirements are applied. . . . . . . .

3.3 Invariant mass distribution of $\mu^{+} \mu^{-}$pairs before and after the confidence level requirement is applied when the dimuons are vertex constrained. . . . . . . . . . . . . . . . . . 
3.4 Normalized invariant mass distribution of $\mu^{+} \mu^{-}$pairs before and after the confidence level requirement is applied when the dimuons are vertex constrained. . . . . . . . . . . . . . . .

3.5 Invariant mass distribution of $\mu^{+} \mu^{-}$and $\mu^{ \pm} \mu^{ \pm}$pairs before and after the confidence level requirement is applied when the dimuons are vertex constrained.

3.6 Normalized invariant mass distribution of $\mu^{+} \mu^{-}$and $\mu^{ \pm} \mu^{ \pm}$pairs before and after the confidence level requirement is applied when the dimuons are vertex constrained. . . . . . . . . . . . . . . 46

3.7 The confidence level distribution of the dimuon vertex fit. . . . . . . 47

3.8 The confidence level distribution of the dimuon mass and vertex fit. . 48

3.9 Level 1 and level 2 low $P_{T}$ central muon trigger efficiency curves . . . 49

3.10 Invariant mass distribution of $K_{s}^{0} \pi^{+}$and $K^{+} \pi^{-}$pairs. . . . . . 51

3.11 Invariant mass distribution of $K^{+} K^{-}$pairs. . . . . . . . 52

3.12 Invariant mass distribution of $\pi^{+} \pi^{-}$pairs before and after the confidence level requirement is applied when the dipions are vertex constrained. . . . . . . . . . . . . . . .

3.13 Normalized invariant mass distribution of $\pi^{+} \pi^{-}$pairs before and after the confidence level requirement is applied when the dipions are vertex

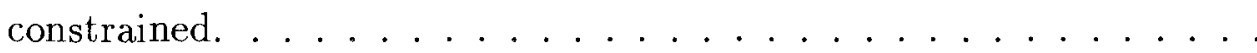

3.14 Invariant mass distribution of $\pi^{+} \pi^{-}$and $\pi^{ \pm} \pi^{ \pm}$pairs before and after the confidence level requirement is applied when the dipions are vertex constrained. . . . . . . . . . . . . . .

3.15 Normalized invariant mass distribution of $\pi^{+} \pi^{-}$and $\pi^{ \pm} \pi^{ \pm}$pairs before and after the confidence level requirement is applied when the dipions are vertex constrained. . . . . . . . . . . . . 56

3.16 The confidence level distribution of the dipion vertex fit. . . . . 56

3.17 The confidence level distribution of the dipion mass and vertex fit. . . 57

3.18 The lifetime distribution of the dipion events that have been vertex

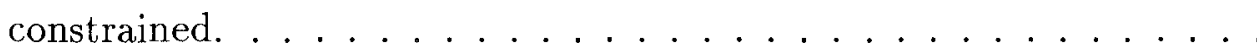

3.19 The lifetime distribution of the dipion events that have been vertex and mass constrained. . . . . . . . . . . . . 
3.20 Invariant mass distribution of $K^{+} K^{-}$pairs before and after the confidence level requirement is applied when the dikaons are vertex constrained. . . . . . . . . . . . . . . .

3.21 Invariant mass distribution of $K^{ \pm} K^{ \pm}$pairs before and after the confidence level requirement is applied when the dikaons are vertex constrained. . . . . . . . . . . . . . . . . . 6 60

3.22 The confidence level distribution of the dikaon vertex fit. . . . . . . 60

3.23 Invariant mass distribution for $B^{+} \rightarrow J / \psi K^{+}$candidate events. . . 65

3.24 Invariant mass distribution for $B^{+} \rightarrow J / \psi K^{*}(892)^{+}$candidate events. $\quad 65$

3.25 Invariant mass distribution for $B^{0} \rightarrow J / \psi K_{s}^{0}$ candidate events. . . . 66

3.26 Invariant mass distribution for $B^{0} \rightarrow J / \psi K^{*}(892)^{0}$ candidate events. $\quad 66$

3.27 Invariant mass distribution for $B_{s}^{0} \rightarrow J / \psi \phi(1020)$ candidate events. . 67

3.28 Invariant mass distribution for $K^{*}(892)^{+}$candidate events taken from

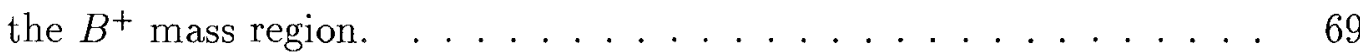

3.29 Invariant mass distribution for $K^{*}(892)^{0}$ candidate events taken from

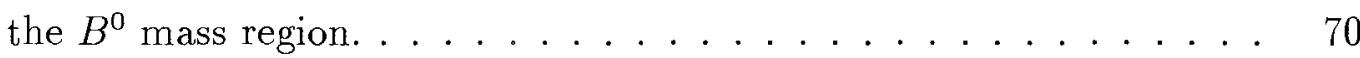

3.30 Invariant mass distribution for $\phi(1020)$ candidate events taken from

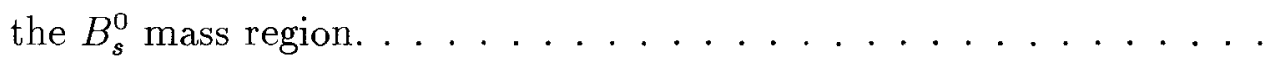

3.31 Invariant mass distribution for $K^{*}(892)^{+}$candidate events taken from

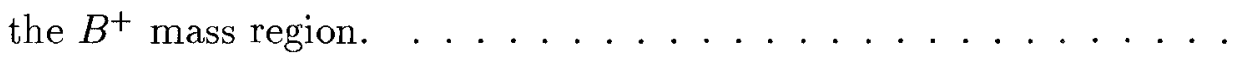

3.32 Invariant mass distribution for $K^{*}(892)^{0}$ candidate events taken from the $B^{0}$ mass region. . . . . . . . . . . . . . .

3.33 Invariant mass distribution for $\phi(1020)$ candidate events taken from

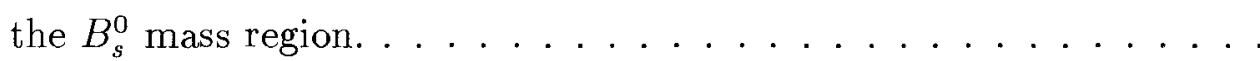

4.1 Track multiplicity distribution for data and Monte Carlo events. . . .

4.2 Transverse momentum distribution of tracks in data and Monte Carlo

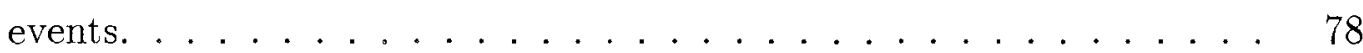

4.3 Total momentum distribution of tracks in data and Monte Carlo events. 78

4.4 Primary $z$ vertex distribution for data and Monte Carlo events. . . . 80

4.5 Primary $z$ vertex uncertainty distribution for data and Monte Carlo

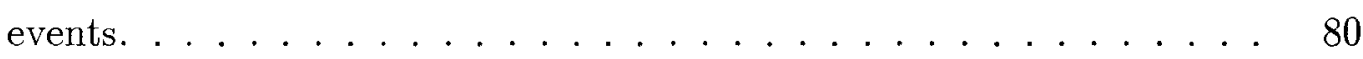

4.6 Primary vertex transverse profile distribution for data and Monte Carlo events. . . . . . . . . . . . . . . . . 
4.7 The confidence level distributions for the $B^{+} \rightarrow J / \psi K^{+}$mass and vertex fit. . . . . . . . . . . . . . . . 88

4.8 The confidence level distributions for the $B^{+} \rightarrow J / \psi K^{*}(892)^{+}$mass and vertex fit. . . . . . . . . . . . . . . . 89

4.9 The confidence level distributions for the $B^{0} \rightarrow J / \psi K_{s}^{0}$ mass and vertex fit. ........................ 89

4.10 The confidence level distribution for the $B^{0} \rightarrow J / \psi K^{*}(892)^{0}$ mass and vertex fit. ..................... . . . 90

4.11 The confidence level distributions for the $B_{s}^{0} \rightarrow J / \psi \phi(1020)$ mass and vertex fit. ............................ 90

6.1 Comparison of ratio of branching fractions and two theoretical predictions for the ratios of branching fractions. . . . . . . . . . . 104 


\section{List of Tables}

1.1 Properties of the known forces. . . . . . . . . . . . . 3

1.2 Masses and charges of the standard model leptons. . . . . . . . . . 4

1.3 Masses and charges of the standard model quarks. . . . . . . . . . 4

1.4 The $b$ quark production cross sections and ratios of collisions that contain $b$ quarks at various accelerators. . . . . . . . . 9

1.5 Branching fraction predictions based on the factorization ansatz. . . 21

2.1 Summary of the geometric coverage and energy resolution of the various CDF calorimeters. . . . . . . . . . . . 30

3.1 Variations of some fitted parameters on the inclusive $J / \psi$ invariant mass spectrum for different SVX requirements. . . . . . . . . .

3.2 Variations of some fitted parameters on the inclusive $K_{s}^{0}$ invariant mass spectrum for different SVX requirements. . . . . . . . . . .

3.3 Optimum transverse momentum cut values for the strange and bottom mesons based on different criteria. . . . . . . . . . . . . . .

3.4 Number of reconstructed $B$ meson decays using different momentum cuts. . . . . . . . . . . . . . . . . .

3.5 Number of reconstructed $B$ mesons decays using similar momentum

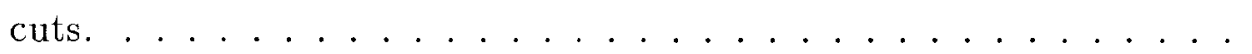

3.6 Definition of the signal and sideband regions for bottom decays with strange mesons that strongly decay. . . . . . . . . . . . . 68

3.7 Efficiencies for the mass window cuts. . . . . . . . . . . . . 69

3.8 Number of reconstructed $B$ meson including non-resonant decays using different momentum cuts. . . . . . . . . . . .

3.9 Number of reconstructed $B$ meson including non-resonant decays using the similar momentum cuts. . . . . . . . . . . . 
4.1 Amount of longitudinal polarization used in the CLEO Monte Carlo calculations. . . . . . . . . . . . . . . . 79

4.2 Branching fractions for strange meson decays. . . . . . . . . . 81

4.3 Kinematic and geometrical acceptances for the decays with different momentum requirements on the bottom and strange mesons. . . . . .

4.4 Kinematic and geometrical acceptances for the decays with similar momentum requirements on the bottom and strange mesons. . . . . . . .

$4.5 c \tau$ standard deviations of sideband events with various number of tracks with SVX hits. . . . . . . . . . . . . .

$4.6 c \tau$ cut efficiency values for the various decays using the central values and one standard deviation of the $c \tau$ values taken from the PDG. . . 83

$4.7 c \tau$ cut efficiency values for $K_{s}^{0}$ mesons using various methods. . . . . 84

4.8 Relative ratio of found events with all tracks exiting the outer CTC can and all found events between Monte Carlo and data. . . . . . 86

4.9 Track finding efficiencies for the different strange mesons. . . . . . . . 86

4.10 Summary of efficiencies for confidence level requirements using the inclusive modes. . . . . . . . . . . . . . . . . . . . .

5.1 Relative percentage change in acceptance with the use of various trigger

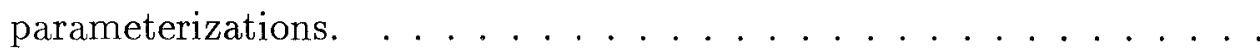

5.2 Efficiencies and systematic uncertainties for the proper decay length requirements. . . . . . . . . . . . . .

5.3 Relative percentage change in acceptance with the use of the various amounts of longitudinal polarization. . . . . . . . . . . . .

5.4 Relative percentage changes of the acceptance with the sets of bottom quark differential production cross sections with different momentum requirements on the bottom and strange mesons. . . . . . . . .

5.5 Relative percentage changes of the acceptance with two sets of bottom quark differential production cross sections with similar momentum requirements on the bottom and strange mesons. . . . . . . . . .

5.6 Invariant masses of the various bottom mesons that were used in the PYTHIA Monte Carlo calculation of detection efficiencies. . . . . . . 96

5.7 Relative percentage changes in geometrical acceptance with the inclusion of excited bottom hadrons. . . . . . . . . . . . . . . . . . 
6.1 Numbers that go into the calculation of the matrix elements $(1,2)$ and $(2,1)$ with different momentum cuts on the bottom and strange mesons. 99

6.2 Various relations for experimental $\gamma$ values. . . . . . . . . . . . 102

6.3 Measured branching fractions values for states involving $J / \psi$ mesons. 106

6.4 World average branching fractions values for states involving $J / \psi$ mesons. 107

6.5 Branching fraction values for the decay $B_{s}^{0} \rightarrow J / \psi \phi(1020)$ as calculated with various $\gamma_{s} / \gamma_{u, d}$ values. The third uncertainty in the branching fraction values represents the uncertainty from the value of $\gamma_{s} / \gamma_{u, d} . \quad .107$

6.6 Measured ratios of light quark production. . . . . . . . . . . 108

6.7 The ratio of strange quark to up or down quark production. . . . . . 109

6.8 Production and decay rates for various decays involving $\Lambda_{b}^{0}$ baryons. . 110

6.9 Branching fractions that are used in the calculation of the $\Lambda_{b}^{0}$ fragmentation fraction. ....................... 111

6.10 Probability to produce $\Lambda_{b}^{0}$ baryons using the LEP semileptonic $\Lambda_{b}^{0}$ decay rates.

6.11 Probabilities to produce the various bottom hadrons species with $f_{u}=$

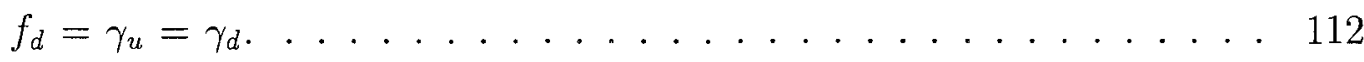

6.12 Probabilities to produce the various bottom hadrons species. . . . . 113

A.1 Number of candidate $B$ meson events using store-averaged beam spot information with $P_{T}^{K}>1.5 \mathrm{GeV} / c$ and $P_{T}^{B}>6.0 \mathrm{GeV} / c \ldots \ldots$

A.2 Number of candidate $B$ meson events using store-averaged beam spot information with $P_{T}^{K}>2.0 \mathrm{GeV} / c$ and $P_{T}^{B}>6.0 \mathrm{GeV} / c \ldots \ldots$

A.3 Number of candidate $B$ meson events using store-averaged beam spot information with $P_{T}^{K}>2.0 \mathrm{GeV} / c$ and $P_{T}^{B}>8.0 \mathrm{GeV} / c \ldots \ldots 120$

A.4 Number of candidate $B$ meson events using event dependent beam spot information with $P_{T}^{K}>1.5 \mathrm{GeV} / c$ and $P_{T}^{B}>6.0 \mathrm{GeV} / c \ldots \ldots 121$

A.5 Number of candidate $B$ meson events using event dependent beam spot information with $P_{T}^{K}>2.0 \mathrm{GeV} / c$ and $P_{T}^{B}>6.0 \mathrm{GeV} / c \ldots \ldots 121$

A.6 Number of candidate $B$ meson events using event dependent beam spot information with $P_{T}^{K}>2.0 \mathrm{GeV} / c$ and $P_{T}^{B}>8.0 \mathrm{GeV} / c \ldots \ldots$

A.7 Gaussian width of candidate $B$ meson signal region using store-average beam spot information with $P_{T}^{K}>1.5 \mathrm{GeV} / c$ and $P_{T}^{\mathbb{B}}>6.0 \mathrm{GeV} / c . .122$

A.8 Gaussian width of candidate $B$ meson signal region using store-average beam spot information with $P_{T}^{K}>2.0 \mathrm{GeV} / c$ and $P_{T}^{B}>6.0 \mathrm{GeV} / c$. . 
A.9 Gaussian width of candidate $B$ meson signal region using store-average beam spot information with $P_{T}^{K}>2.0 \mathrm{GeV} / c$ and $P_{T}^{B}>8.0 \mathrm{GeV} / c$. .

A.10 Gaussian width of candidate $B$ meson signal region using event dependent beam spot information with $P_{T}^{K}>1.5 \mathrm{GeV} / c$ and $P_{T}^{B}>6.0$ $\mathrm{GeV} / c \ldots \ldots \ldots \ldots \ldots \ldots \ldots \ldots$

A.11 Gaussian width of candidate $B$ meson signal region using event dependent beam spot information with $P_{T}^{K}>2.0 \mathrm{GeV} / c$ and $P_{T}^{B}>6.0$ $\mathrm{GeV} / c \ldots \ldots \ldots \ldots \ldots \ldots \ldots \ldots$

A.12 Gaussian width of candidate $B$ meson signal region using event dependent beam spot information with $P_{T}^{K}>2.0 \mathrm{GeV} / c$ and $P_{T}^{B}>8.0$

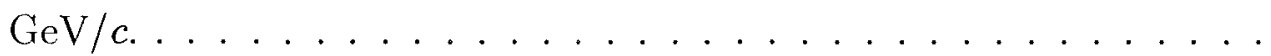

A.13 Ratio of candidate $B$ meson signal to background using store-averaged beam spot information with $P_{T}^{K}>1.5 \mathrm{GeV} / c$ and $P_{T}^{B}>6.0 \mathrm{GeV} / c$. .

A.14 Ratio of candidate $B$ meson signal to background using store-averaged beam spot information with $P_{T}^{K}>2.0 \mathrm{GeV} / c$ and $P_{T}^{B}>6.0 \mathrm{GeV} / c$. .

A.15 Ratio of candidate $B$ meson signal to background using store-averaged beam spot information with $P_{T}^{K}>2.0 \mathrm{GeV} / c$ and $P_{T}^{B}>8.0 \mathrm{GeV} / c$. . 124

A.16 Ratio of candidate $B$ meson signal to background using event dependent beam spot information with $P_{T}^{K}>1.5 \mathrm{GeV} / c$ and $P_{T}^{B}>6.0 \mathrm{GeV} / c .125$

A.17 Ratio of candidate $B$ meson signal to background using event dependent beam spot information with $P_{T}^{K}>2.0 \mathrm{GeV} / c$ and $P_{T}^{B}>6.0 \mathrm{GeV} / c .125$

A.18 Ratio of candidate $B$ meson signal to background using event dependent beam spot information with $P_{T}^{K}>2.0 \mathrm{GeV} / c$ and $P_{T}^{B}>8.0 \mathrm{GeV} / c .125$

A.19 Significance of candidate $B$ meson signal using store-averaged beam spot information with $P_{T}^{K}>1.5 \mathrm{GeV} / c$ and $P_{T}^{B}>6.0 \mathrm{GeV} / c . \ldots 126$

A.20 Significance of candidate $B$ meson signal using store-averaged beam spot information with $P_{T}^{K}>2.0 \mathrm{GeV} / c$ and $P_{T}^{B}>6.0 \mathrm{GeV} / c . \ldots 126$

A.21 Significance of candidate $B$ meson signal using store-averaged beam spot information with $P_{T}^{K}>2.0 \mathrm{GeV} / c$ and $P_{T}^{B}>8.0 \mathrm{GeV} / c . \ldots 126$

A.22 Significance of candidate $B$ meson signal using event dependent beam spot information with $P_{T}^{K}>1.5 \mathrm{GeV} / c$ and $P_{T}^{B}>6.0 \mathrm{GeV} / c$. . .

A.23 Significance of candidate $B$ meson signal using event dependent beam spot information with $P_{T}^{K}>2.0 \mathrm{GeV} / c$ and $P_{T}^{B}>6.0 \mathrm{GeV} / c$. . . 
A.24 Significance of candidate $B$ meson signal using event dependent beam spot information with $P_{T}^{K}>2.0 \mathrm{GeV} / c$ and $P_{T}^{B}>8.0 \mathrm{GeV} / c . \cdots 127$

B.1 Numbers that go into the calculation of the matrix elements $(1,2)$ and $(2,1)$ with different momentum cuts on the bottom and strange mesons. 129

B.2 Numbers that go into the calculation of the matrix elements $(1,2)$ and $(2,1)$ with same momentum cuts on the bottom and strange mesons. .

B.3 Numbers that go into the calculation of the matrix elements $(1,3)$ and $(3,1)$ with different momentum cuts on the bottom and strange mesons. 131

B.4 Numbers that go into the calculation of the matrix elements $(1,3)$ and $(3,1)$ with same momentum cuts on the bottom and strange mesons. . 132

B.5 Numbers that go into the calculation of the matrix elements $(1,4)$ and $(4,1)$ with different momentum cuts on the bottom and strange mesons. 133

B.6 Numbers that go into the calculation of the matrix elements $(1,4)$ and $(4,1)$ with same momentum cuts on the bottom and strange mesons. . 133

B.7 Numbers that go into the calculation of the matrix elements $(1,5)$ and $(5,1)$ with different momentum cuts on the bottom and strange mesons. 134

B.8 Numbers that go into the calculation of the matrix elements $(1,5)$ and $(5,1)$ with same momentum cuts on the bottom and strange mesons. . 135

B.9 Numbers that go into the calculation of the matrix elements $(2,3)$ and $(3,2)$ with different momentum cuts on the bottom and strange mesons. 135 B.10 Numbers that go into the calculation of the matrix elements $(2,3)$ and $(3,2)$ with same momentum cuts on the bottom and strange mesons. . 136

B.11 Numbers that go into the calculation of the matrix elements $(2,4)$ and $(4,2)$ with different momentum cuts on the bottom and strange mesons. 137 B.12 Numbers that go into the calculation of the matrix elements $(2,4)$ and $(4,2)$ with same momentum cuts on the bottom and strange mesons. . 138 B.13 Numbers that go into the calculation of the matrix elements $(2,5)$ and $(5,2)$ with different momentum cuts on the bottom and strange mesons. 139 B.14 Numbers that go into the calculation of the matrix elements $(2,5)$ and $(5,2)$ with same momentum cuts on the bottom and strange mesons. . 140 B.15 Numbers that go into the calculation of the matrix elements $(3,4)$ and $(4,3)$ with different momentum cuts on the bottom and strange mesons. 141 B.16 Numbers that go into the calculation of the matrix elements $(3,4)$ and $(4,3)$ with same momentum cuts on the bottom and strange mesons. . 142 
B.17 Numbers that go into the calculation of the matrix elements $(3,5)$ and $(5,3)$ with different momentum cuts on the bottom and strange mesons. 143 B.18 Numbers that go into the calculation of the matrix elements $(3,5)$ and $(5,3)$ with same momentum cuts on the bottom and strange mesons. . 144 B.19 Numbers that go into the calculation of the matrix elements $(4,5)$ and $(5,4)$ with different momentum cuts on the bottom and strange mesons. 145 B.20 Numbers that go into the calculation of the matrix elements $(4,5)$ and $(5,4)$ with same momentum cuts on the bottom and strange mesons. . 146 


\section{Chapter 1}

\section{Introduction}

This thesis involves the study of three types of bottom $(B)$, or beauty mesons. In particular, two aspects of the three different types of bottom mesons were investigated. The first of these was the determination of the branching fractions $(\mathcal{B})$ of the $B^{+}, B^{0}$ and $B_{s}^{0}$ mesons (as well as the conjugate states) into states involving a $J / \psi$ meson. The second involved a measurement of the relative production rates of the three different types of bottom mesons.

For this study, the bottom mesons were produced by colliding protons and antiprotons at a center-of-mass energy of $1.8 \mathrm{TeV}$ with the use of the Tevatron Collider and detected with the Collider Detector at Fermilab (CDF). The theory that describes the production and decay of bottom mesons consists of Quantum Chromodynamics (QCD) [1], the standard model of electroweak interactions $[2,3,4]$ and the quark model $[5,6]$. Together, these models, developed over the last thirty years, form the basis for the theory of elementary particle physics. By studying the production and decay properties of bottom mesons, one can test various aspects of these theories.

The theoretical aspects of the production and decay of bottom mesons are discussed in the later sections of this chapter. The Tevatron collider and the CDF detector are described in Chapter 2. The data reconstruction and selection procedures are discussed in Chapter 3. The acceptance and efficiency corrections for the various bottom meson decays are described in Chapter 4. A discussion of systematic uncertainties is given in Chapter 5. The results are presented in Chapter 6 and concluding remarks are given in Chapter 7 . 


\section{$1.1 \quad$ Historical Overview}

The field of elementary particle physics deals with the study of the fundamental constituents of matter and how they interact with each other. At the beginning of the twentieth century atoms were thought to be the fundamental building blocks of matter. Over the years many different types of atoms were discovered. At last count there were 112 known atomic elements. The proliferation of elements suggested that the atomic elements were not fundamental. This was confirmed by Ernest Rutherford in 1911 with the discovery of the nucleus. Eight years later, Rutherford succeeded in breaking up a nitrogen nucleus with the emission of a hydrogen nucleus that he called the proton. At that time, protons and electrons (discovered by J. J. Thomson in 1897), were thought to be the fundamental building blocks of matter.

By the mid sixties there was a deluge of fundamental particles including many different types of leptons (electrons, muons and neutrinos), hadrons (protons, neutrons, pions and kaons) and bosons (photons). As before, the proliferation of these particle types hinted that they consisted of even more fundamental particles. In 1964, M. Gell-Mann [5] and G. Zweig [6] independently proposed that hadrons were made of smaller fundamental particles called quarks. Their quark model proposed that combinations of two and three quarks combine to form the observed hadrons. At that time, three quark flavors (with three antiquark flavors) were needed to accommodate all of the known hadrons. Since then, the number of known quarks has increased to 6 (with 6 antiquarks). None of these exist as free particles but in combinations of two or three quarks. The leptons and bosons are still considered fundamental particles, but there are currently 6 leptons (with 6 antileptons) and 12 bosons, eight of which are called gluons. All these are believed to be fundamental particles, that is, they do not have any substructure. It is these 36 particles (including antiparticles) that form the building blocks of the standard model of fundamental particles and interactions.

\subsection{The Standard Model}

In the standard model, the quarks and leptons are spin $1 / 2$ fermions that obey FermiDirac statistics. These particles interact with the exchange of spin 1 bosons (photons, $W^{ \pm}, Z^{0}$ and gluons) that obey Bose-Einstein statistics. The bosons act as the mediators of the various forces. Photons $(\gamma)$ are the mediators of the electromagnetic 


\begin{tabular}{|c|c|c|c|c|}
\hline Property/Force & Strong & Electromagnetic & Weak & Gravitation \\
\hline \hline Mediating Particle & Eight Gluons & Photon & $W^{ \pm}$and $Z^{0}$ & Graviton \\
\hline $\begin{array}{c}\text { Particles } \\
\text { Experiencing } \\
\text { Force }\end{array}$ & $\begin{array}{c}\text { Quarks } \\
\text { and } \\
\text { Gluons }\end{array}$ & $\begin{array}{c}\text { Charged } \\
\text { Particles }\end{array}$ & $\begin{array}{c}\text { Quarks } \\
\text { and } \\
\text { Leptons }\end{array}$ & $\begin{array}{c}\text { All } \\
\text { Particles }\end{array}$ \\
\hline Relative Strength & 25 & 1 & 0.8 & $10^{-41}$ \\
\hline Mediator Mass & 0 & 0 & $80.22,91.19$ & 0 \\
\hline Range & $\leq 10^{-15}$ & $\infty$ & $10^{-18}$ & $\infty$ \\
\hline \hline
\end{tabular}

Table 1.1: Properties of the known forces taken from Ref $[7,8]$. Masses are in $\mathrm{GeV} / c^{2}$ and ranges are in meters. Gravity is not part of the standard model but is included for the sake of comparison. Gravitons have yet to be discovered.

force, the $W^{ \pm}$and $Z^{0}$ particles are the mediators of the weak force, and the eight gluons $(\mathrm{g})$ are the mediators of the strong force. Some properties of the known forces are summarized in Table 1.1.

The leptons appear to be point particles that carry integral electric charge. Half of the leptons are electrically neutral. These neutral leptons are called neutrinos and have very small or zero mass. The other leptons have electric charges of $\pm e$. The negatively charged particles are the electrons, muons and tau leptons. The antiparticles have the opposite charge and are called positrons $\left(e^{+}\right)$, antimuons $\left(\mu^{+}\right)$and antitaus $\left(\tau^{+}\right)$. The mass and charge of the leptons are summarized in Table 1.2. The antiparticles are not listed in Table 1.2, but have the same mass and opposite charge. For this analysis, the only relevant leptons are the muons and their antiparticles.

As in the case of the leptons, quarks are considered point particles. But unlike the leptons, they are not free. The quarks are confined within mesons or baryons, which are collectively known as hadrons. There are six types (flavors) of quarks with the various charges and masses listed in Table 1.3.

In the quark model, baryons are three quark $(q q q$ or $\overline{q q q})$ states. The quarks within a baryon can be of any flavor and the baryon wavefunction has the following form

$$
\left|q q q>_{A}=\right| \text { color }>_{\mathrm{A}} \times \mid \text { Space, Spin, Flavor }>_{\mathrm{S}} \text {, }
$$

where the subscripts $\mathrm{A}$ and $\mathrm{S}$ indicate antisymmetric and symmetric states, respec- 


\begin{tabular}{|c|c|c|}
\hline \hline Particle & Mass $\left(\mathrm{MeV} / \mathrm{c}^{2}\right)$ & Charge \\
\hline \hline$\nu_{e}$ (electron neutrino) & $<7.2 \times 10^{-6}$ & 0 \\
\hline$e^{-}$(electron) & 0.511 & -1 \\
\hline \hline$\nu_{\mu}$ (muon neutrino) & $<0.27$ & 0 \\
\hline$\mu^{-}$(muon) & 105.7 & -1 \\
\hline \hline$\nu_{\tau}$ (tau neutrino) & $<24$ & 0 \\
\hline$\tau^{-}$(tau) & 1777.1 & -1 \\
\hline \hline
\end{tabular}

Table 1.2: Masses and charges of the standard model leptons. The limits on the electron and tau neutrino mass are given at the 95\% confidence level and the limit on the muon neutrino is given at the $90 \%$ confidence level. The tau neutrino limit is taken from Ref [9]; the other values are taken from Ref [7]. The lepton charges are given in units of the absolute electron charge.

\begin{tabular}{|c|c|c|}
\hline \hline Quark Flavor & Mass $\left(\mathrm{MeV} / \mathrm{c}^{2}\right)$ & Charge \\
\hline \hline$u$ (up) & 2 to 8 & $+\frac{2}{3}$ \\
\hline$d$ (down) & 5 to 15 & $-\frac{1}{3}$ \\
\hline \hline$c$ (charm) & 1000 to 1600 & $+\frac{2}{3}$ \\
\hline$s$ (strange) & 100 to 300 & $-\frac{1}{3}$ \\
\hline \hline$t$ (top) & $(1.76 \pm 0.08$ (stat) \pm 0.10 (syst) $) \times 10^{5}$ & $+\frac{2}{3}$ \\
\hline$b$ (bottom) & 4100 to 4500 & $-\frac{1}{3}$ \\
\hline \hline
\end{tabular}

Table 1.3: Masses and charges of the standard model quarks. The listed mass ranges are taken from Particle Data Group [7] and the top mass is taken from Ref [10]. The antiparticles are denoted with a bar on top and have the opposite charge. 
tively.

Color is a quantum number that was introduced to make the wavefunction of the $\Delta^{++}$(uuu) baryon antisymmetric under the interchange of any two identical quarks as required by the Pauli exclusion principle. Quarks can carry one of three colors and the antiquarks can carry one of the three anticolors. Since particles with non-zero color have not been observed, the baryon wavefunctions are required to be colorless. In other words, all three colors must be present in the baryon wavefunction. This color quantum number is the basis of an $\mathrm{SU}(3)$ gauge theory for the strong quark interactions called Quantum Chromodynamics.

In QCD, the interquark interactions are assumed to be invariant under color interchange. The particles mediating the quark-quark interactions are called gluons and are postulated to belong to an octet representation of the symmetry group $\mathrm{SU}(3)$.

Mesons are bound states of a quark and an antiquark ( $q \bar{q}$ states). As in the case of baryons, the quarks within a meson can be of any flavor. The requirement that the mesons be colorless constrains the color wavefunction to consist of color and anticolor quark pairs and to be symmetric.

The parity of a meson is given by $(-1)^{L+1}$, where $L$ is the orbital angular momentum between the two quarks. In addition to different orbital angular momentum states, there can be radial excited states. Thus, the mesons can be categorized into $N^{2 S+1} L_{J}$ states, where $N$ is the radial excitation level, $S$ is the total spin and $J$ is the total angular momentum.

All of the bottom meson states investigated in this analysis have $S=0, L=0$, $J=0$ and $N=0$ but have different quark compositions. $B^{+}$mesons consist of $u \bar{b}$ quark pairs, $B^{0}$ mesons consist of $d \bar{b}$ quark pairs, and $B_{s}^{0}$ mesons consist of $s \bar{b}$ quark pairs.

\subsection{Production of Bottom Quarks}

The production of bottom quarks in $\bar{p} p$ collisions can be described by QCD as the scattering of partons (quarks and/or gluons) within the protons and antiprotons. The parton model formula for the inclusive production of a heavy quark $(Q)$ of momentum $\mathrm{p}$ and of energy $E$ in a hadron-hadron collision $H_{A}\left(P_{A}\right)+H_{B}\left(P_{B}\right) \rightarrow Q(\mathrm{p})+X$ is given by 


$$
\frac{E d^{3} \sigma}{d^{3} \mathrm{p}}=\sum_{i j} \int d x_{1} d x_{2}\left(\frac{E d^{3} \hat{\sigma}_{i j}\left(x_{1} P_{A}, x_{2} P_{B}, \mathrm{p} ; m_{\mathrm{Q}}, \mu, \Lambda\right)}{d^{3} \mathrm{p}}\right) F_{i}^{A}\left(x_{1}, \mu\right) F_{j}^{B}\left(x_{2}, \mu\right),
$$

where $x_{1} P_{A}$, and $x_{2} P_{B}$ are the momenta of the incoming partons, $P_{A}$ and $P_{B}$ are the momenta of the colliding hadrons $H_{A}$ and $H_{B}, \hat{\sigma}_{i j}$ is the parton-level cross section, $m_{\mathrm{Q}}$ is the mass of the heavy quark produced, $\mu$ is a parameter related to the energy scale of the reaction, and $\Lambda$ determines the coupling strength $\alpha_{s}$. The sum is over all the partons in the two colliding hadrons. The structure function $F_{i}^{A}\left(x_{1}, Q\right)\left(F_{j}^{B}\left(x_{2}, Q\right)\right)$ gives the probability density for a parton of type $i(j)$ and momentum fraction $x_{1}\left(x_{2}\right)$ to be found in the hadron $H_{A}\left(H_{B}\right)$ when it is probed at a four momentum transfer $Q$. The variable $Q$ is usually taken to be equal to $\mu$. The relation between $\alpha_{s}, \mu$ and $\Lambda$ is given by

$$
\alpha_{s}(\mu)=\frac{1}{b_{f} \ln \left(\mu^{2} / \Lambda_{f}^{2}\right)}\left(1-\frac{b_{f}^{\prime} \ln \left[\ln \left(\mu^{2} / \Lambda_{f}^{2}\right)\right]}{b_{f} \ln \left(\mu^{2} / \Lambda_{f}^{2}\right)}\right)+O\left(\frac{\ln ^{2}\left[\ln \left(\mu^{2} / \Lambda_{f}^{2}\right)\right]}{\ln ^{3}\left(\mu^{2} / \Lambda_{f}^{2}\right)}\right)
$$

with

$$
b_{f}=\frac{33-2 f}{12 \pi}, \quad b_{f}^{\prime}=\frac{153-19 f}{2 \pi(33-2 f)},
$$

and $\Lambda=\Lambda_{f}$, where $f$ is the number of quark flavors that are active. The variables $m_{\mathrm{Q}}, \mu$ and $\Lambda$ are parameters of the theory and are determined from experimental data. In principle, the $\mu$ in $\hat{\sigma}_{i j}$ and the $\mu$ in $F_{i}^{A}$ and $F_{j}^{B}$ can be different, but are usually taken to be equal. An important thing to note about the scale parameter $\mu$ is that its most appropriate value is not known a priori. It is generally taken to be equal to the "physical" scale (e.g., the mass of the produced quark $m_{\mathrm{Q}}$ or the transverse mass $\sqrt{m_{\mathrm{Q}}^{2}+p_{T}^{2}}$ ) in order to reduce the sensitivity of the calculation to any truncations in the perturbation expansion. The uncertainty in the value of $\mu$ is a major source of uncertainty in the prediction of the cross section for heavy quark production.

The parton-level cross sections $\left(\hat{\sigma}_{i j}\right)$ have been calculated by Nason, Dawson and Ellis (NDE) to order $\alpha_{s}^{3}[11,12]$. Their calculations include virtual and real radiative corrections to the $\mathrm{O}\left(\alpha_{s}^{3}\right)$ matrix elements. Some Feynman diagrams that contribute to $O\left(\alpha_{s}^{2}\right)$ and $O\left(\alpha_{s}^{3}\right)$ are shown in Figures 1.1 and 1.2, respectively. An important result of the higher order calculation is that the processes 

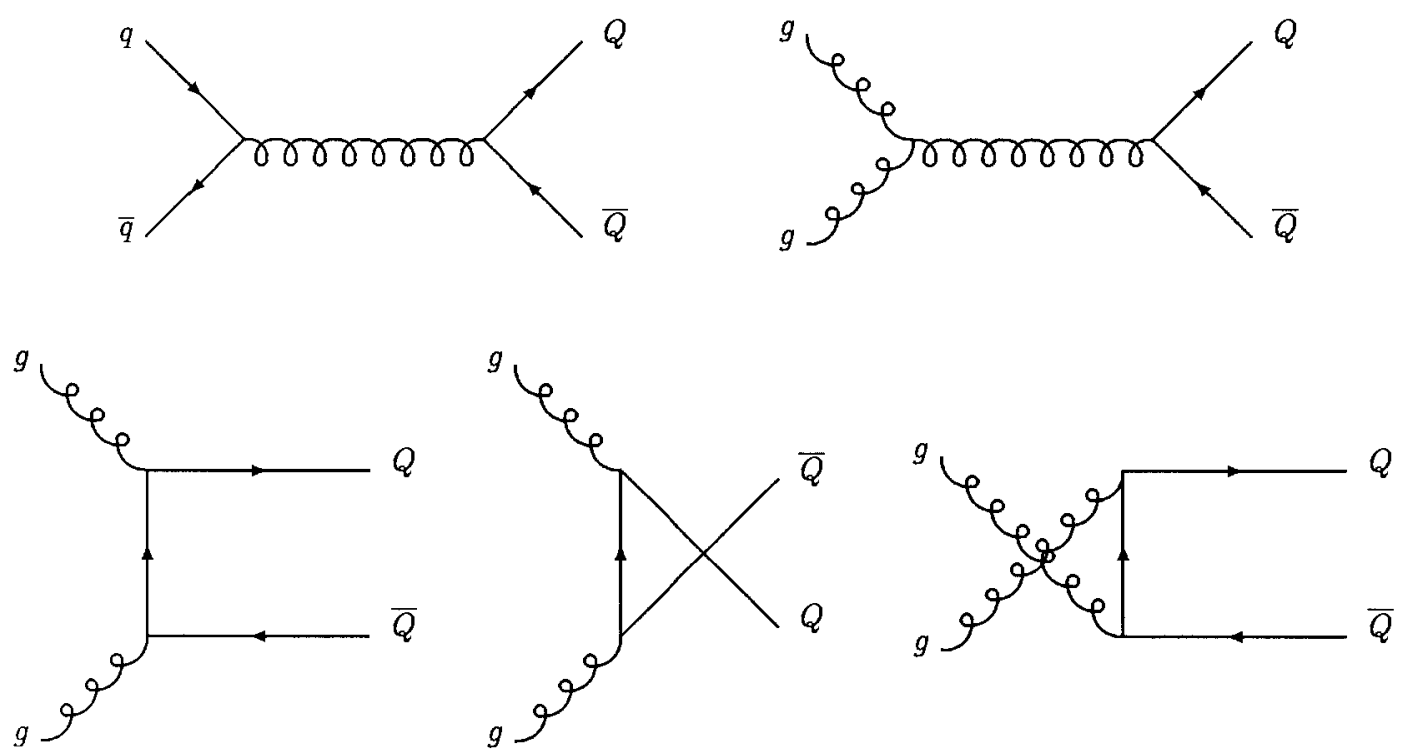

Figure 1.1: The lowest order Feynman diagrams for quark production in hadron colliders.

$$
g g \rightarrow Q \bar{Q} g, \quad g q \rightarrow Q \bar{Q} q, \quad g \bar{q} \rightarrow Q \bar{Q} \bar{q},
$$

which contribute to $O\left(\alpha_{s}^{3}\right)$, dominate at large values of partonic center-of-mass energy $(\hat{s})$. As a result of this, the production cross section will increase at large values of $\hat{s}$ when going from calculations of order $O\left(\alpha_{s}^{2}\right)$ to $O\left(\alpha_{s}^{3}\right)$. This increase can be explained by the following calculation. For the process $(g+g \rightarrow g+Q+\bar{Q})$, the cross section $\hat{\sigma}(g g \rightarrow g Q \bar{Q})$ can be approximated by $\hat{\sigma}(g g \rightarrow g g) P\left(\hat{s}, m_{\mathrm{Q}}\right)$ where $P\left(\hat{s}, m_{\mathrm{Q}}\right)$ is the probability for the branching process $g \rightarrow Q \bar{Q}$. Then the ratio of cross sections for the $O\left(\alpha_{s}^{3}\right)$ and $O\left(\alpha_{s}^{2}\right)$ terms is approximately given by

$$
\frac{\hat{\sigma}(g g \rightarrow g Q \bar{Q})}{\hat{\sigma}(g g \rightarrow Q \bar{Q})} \approx \frac{\hat{\sigma}(g g \rightarrow g g)}{\hat{\sigma}(g g \rightarrow Q \bar{Q})} P\left(\hat{s}, m_{\mathrm{Q}}\right) .
$$

For $p_{T} \gg m_{\mathrm{Q}}$ the right-hand cross section ratio in Equation 1.6 is $\sim 100$ [13] and the branching probability $P\left(\hat{s}, m_{\mathrm{Q}}\right)$ is $\sim \alpha_{s}$, so

$$
\frac{\hat{\sigma}(g g \rightarrow g Q \bar{Q})}{\hat{\sigma}(g g \rightarrow Q \bar{Q})} \sim 100 \times \alpha_{s} \sim 10 .
$$

Therefore, the effects of $O\left(\alpha_{s}^{3}\right)$ terms in the calculation of the production cross sections for heavy quarks are non-negligible. 

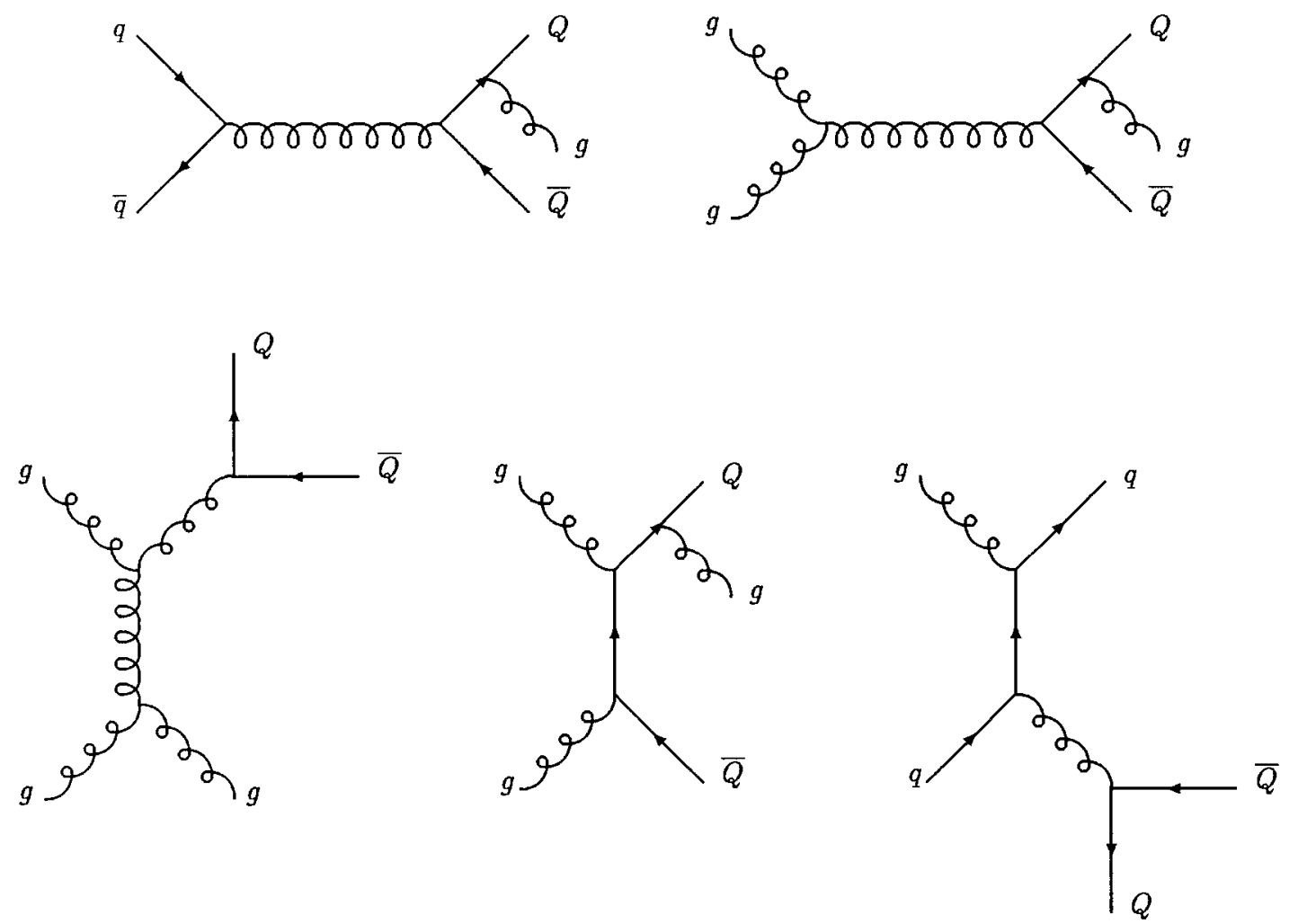

Figure 1.2: Some Feynman diagrams that contribute in order of $\alpha_{s}^{3}$ in the parton cross section. 


\begin{tabular}{|l|c|c|}
\hline \hline Accelerator & $\sigma_{\bar{b} b}^{\max }(\mu \mathrm{b})$ & $\sigma_{\text {tot }} / \sigma_{\bar{b} b}$ \\
\hline \hline CESR & $\sim 1.1 \times 10^{-3}$ & $3-4$ \\
\hline LEP & $\sim 9 \times 10^{-3}$ & $\sim 5$ \\
\hline Tevatron & $20-40$ & 2500 \\
\hline LHC & $\sim 200$ & 500 \\
\hline \hline
\end{tabular}

Table 1.4: The $b$ quark production cross sections and ratios of collisions that contain $b$ quarks at various accelerators. The results for the future LHC accelerator are based on Monte Carlo calculations [19].

The resulting $b$ quark production cross section from $\bar{p} p$ collisions at $\sqrt{s}=1.8 \mathrm{TeV}$ as a function of minimum transverse momentum of the produced quark is shown in Figure 1.3. The parton distribution functions were taken from Ref [14] and the calculation was done for the case in which the absolute rapidity of the produced quark was less than one. The rapidity $(y)$ of the quark is given by

$$
y=\frac{1}{2} \ln \left(\frac{E+p_{Z}}{E-p_{Z}}\right),
$$

where $E$ is the energy of the quark and $p_{Z}$ is the momentum of the quark along the axis of the proton-antiproton collision. As Figure 1.3 illustrates, the shape of the predicted $b$ quark production cross section at $\sqrt{s}=1.8 \mathrm{TeV}$ agrees with the data but it underestimates the production cross section by a factor of between two and four [15]. An interesting thing to note is the size of the production cross section. The bottom quark production cross section at the Tevatron is more than three orders of magnitude higher than at other current accelerators that produce bottom hadrons. However, this advantage is offset by the even larger cross section to produce light quarks and gluons. The relative cross sections for some of the current and future accelerators that are used to study various aspects of bottom physics are given in Table 1.4. The problem of having only a small fraction of the $\bar{p} p$ collisions produce bottom quarks is overcome by "triggering" on those events that have properties consistent with those of the bottom hadrons that one is interested in studying. For this analysis, the requirement of two muons with an invariant mass close to the $J / \psi$ mass rejects a large amount of background but is highly efficient in selecting decays of bottom mesons to states involving $J / \psi$ mesons. 


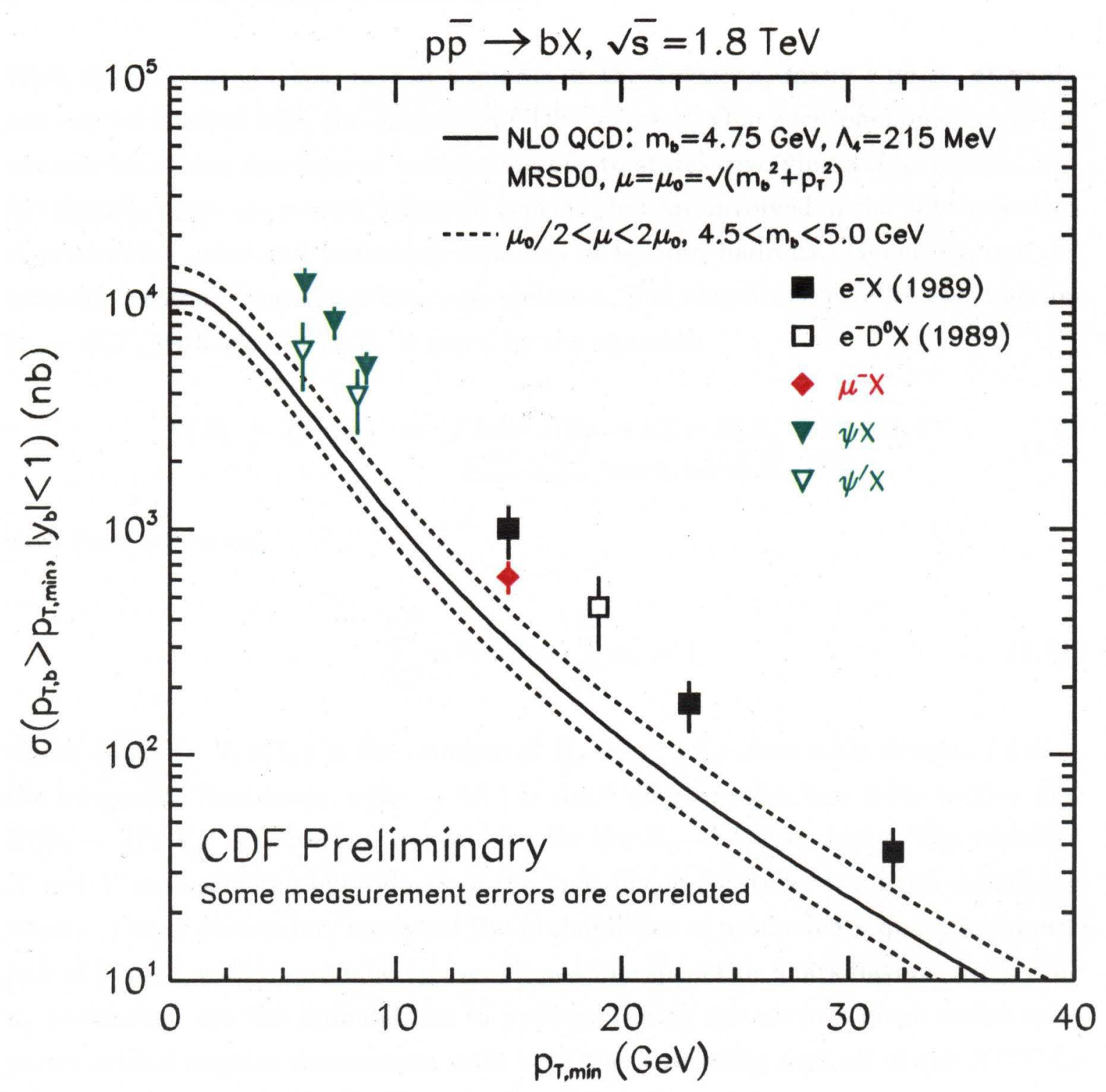

Figure 1.3: The $b$ quark production cross section from $\bar{p} p$ collisions at a center-of-mass energy of $1.8 \mathrm{TeV}$ for a transverse momentum above $p_{T, \min }$ and absolute rapidity less than 1 . The solid line is the next to leading order (NLO) NDE prediction $[11,12,16]$ using the $\mathrm{MRSD}_{0}$ parton distribution function [14] and the Peterson parameterization for $b$ quark fragmentation $[17,18]$. The dotted curves are estimates of the theoretical systematic uncertainties obtained by varying the choice of the scale $\mu$ and the mass of the $b$ quark. The data were obtained at CDF with the assumption that $75 \%$ of the $b$ quarks fragment equally into $B^{+}$and $B^{0}$ mesons. 


\subsection{Hadronization of Bottom Quarks and Decay of Bottom Hadrons}

With the large production rate of $b$ quarks at the Tevatron, many $b$ quark phenomena can be studied with the use of the CDF detector. One such phenomenon is the relative branching fractions of bottom mesons to states involving a $J / \psi$ meson. Unfortunately, there are several unknown aspects that are involved in the determination of production rates and branching fractions of bottom hadrons. Generally, one detects the hadrons from the primary $\bar{p} p$ collision. The observable rate for the reaction $\bar{p} p \rightarrow B_{q} Y$, with $B_{q} \rightarrow J / \psi X_{q}$ is given by the equation

$$
\begin{aligned}
N\left(B_{q} \rightarrow J / \psi X_{q}\right)= & \int L d t \cdot \sigma(\bar{p} p \rightarrow b X) \cdot \mathcal{B}\left(B_{q} \rightarrow J / \psi X_{q}\right) \\
& \cdot \sum_{i=1}^{S . F} \sum_{j=1}^{S . P} \gamma_{i} \alpha_{j} \epsilon_{i j}\left(J / \psi, X_{q}, \mathcal{B}^{i j}\right)
\end{aligned}
$$

with the constraints

$$
\sum_{i=1}^{S . F} \gamma_{i}=1 \text { and } \sum_{j=1}^{S . P} \alpha_{j}=1
$$

where $N\left(B_{q} \rightarrow J / \psi X_{q}\right)$ is the number of $B_{q} \rightarrow J / \psi X_{q}$ observable decays, $\int L d t$ is the integrated luminosity, $\sigma(\bar{p} p \rightarrow b X)$ is the $b$ quark production cross section and $\mathcal{B}\left(B_{q} \rightarrow J / \psi X_{q}\right)$ is the branching fraction for the $B_{q} \rightarrow J / \psi X_{q}$ decay. The variables $X$ and $Y$ represent the byproducts of the $\bar{p} p$ collision (particles from the underlying event). The $\gamma_{i}$ parameters represent the probabilities of producing a quark antiquark pair of flavor $i$, with the sum being over all possible spectator quark flavors $(S . F)$. The $\alpha_{j}$ parameters are the probabilities to produce the $\bar{q} q$ system in a given radial-spinparity-orbital angular momentum state with the sum being over all of the $N^{2 S+1} L_{J}$ states $(S . P)$ and $\epsilon_{i j}\left(J / \psi, X_{q}, \mathcal{B}^{i j}\right)$ are the efficiencies to detect the hadron in a given radial-spin-parity-orbital angular momentum state (including all of the branching fractions involved in the decay except for the one representing the decay that is being studied). It should be noted that $\gamma_{i}$ and $\alpha_{j}$ could depend on the center-of-mass energy and the type of beam particles used to create the hadrons. It is assumed that $\gamma_{i}$ and $\alpha_{j}$ are independent and factorizable.

Sometimes Equation 1.9 is written with $f_{i}$ 's (fragmentation fractions) instead of the $\gamma_{i}$ 's and $\alpha_{i}$ 's, with the two being related by the equation 


$$
f_{i} \cdot \epsilon_{i}\left(J / \psi, X_{q}, \mathcal{B}^{i}\right) \sim \gamma_{i} \cdot \sum_{j=1}^{S . P} \alpha_{j} \cdot \epsilon_{i j}\left(J / \psi, X_{q}, \mathcal{B}^{i j}\right),
$$

where the $f_{i}$ 's represent the probabilities to directly produce a bottom hadron containing a spectator quark with a flavor $i$, or to produce a bottom hadron containing a spectator quark with flavor $i$ through the decay of a bottom hadron with a spectator quark of any flavor. The two sides in Equation 1.11 are not equal in principle, because the efficiencies are not necessarily the same.

Many of the quantities introduced above are not known or are poorly measured. The quantities that are known are the number of observed decays, integrated luminosity and detection efficiencies. The efficiency values depend on the partially known $b$ quark differential production cross section, but the uncertainty this creates in $\epsilon_{i j}$ can be reduced, as well as removing the $\sigma(\bar{p} p \rightarrow b X)$ term, by taking ratios of Equation 1.9 for different hadronic decays. The efficiency is then sensitive to only the shape of the $b$ quark differential production cross section. Taking the ratios of Equation 1.9 also has the advantage that the integrated luminosity term cancels out.

The $\gamma_{i}$ values are important since they come into play in almost all aspects of strange, charm and bottom physics. Generally, $\gamma_{u}$ is taken to be equal to $\gamma_{d}$ because the $u$ and $d$ quark masses are almost identical. This is not true for strange and charm quarks because their masses are very different from the other quark masses. One must either measure the $\gamma_{i}$ values or make reasonable assumptions. A method to determine the $\gamma_{i}$ values will be discussed later. For now a reasonable calculation following the methods shown in references $[20,21,22]$ will suffice. Given a constant vector electric field in space and time, the rate of production per unit volume per unit time of a pair of electrons according to the Dirac equation is proportional to

$$
(F / 2 \pi) \exp \left(-\pi\left(m^{2}+k_{\perp}^{2}\right) / F\right) d^{2} k_{\perp} /(2 \pi)^{2},
$$

where $F$ is the force field ( $F=e E, E$ is the electric field and $e$ is the charge of the particle), $m$ is the mass of the electron, and $k_{\perp}$ is the momentum of the electrons transverse to the direction of the field. In an analogous way, this model can be extended to the production of quark pairs by replacing the mass of the electrons with the mass of the quarks and replacing the electric force field with the color force field between quarks. The color force field between two quarks is approximately $1 \mathrm{GeV} / \mathrm{fm}$ or $0.2 \mathrm{GeV}^{2}$ [8]. Taking the bare quark masses from the PDG [7] and the spectator 
baryon quark composite mass to be $\left(\left|M\left(\Lambda_{b}^{0}\right)-M\left(B^{+}\right)\right|\right)$, one finds the following relative $\gamma$ values for the lower, average and higher PDG quark masses:

$$
\begin{aligned}
& B^{+}: B^{0}: B_{s}^{0}: B_{c}^{+}: \text {Baryons }=0.335: 0.335: 0.286: 5.05 \times 10^{-8}: 0.044 \\
& B^{+}: B^{0}: B_{s}^{0}: B_{c}^{+}: \text {Baryons }=0.375: 0.375: 0.200: 1.11 \times 10^{-12}: 0.050 \\
& B^{+}: B^{0}: B_{s}^{0}: B_{c}^{+}: \text {Baryons }=0.422: 0.422: 0.103: 1.45 \times 10^{-18}: 0.055
\end{aligned}
$$

It should be noted that for measurements that depend on the rate of $B^{0}$ and $B_{s}^{0}$ production, the Particle Data Group [7] uses 0.391 and 0.117 for the respective fragmentation fractions.

Treating the spectator diquarks in a baryon as one quark is a very rough approximation. Other models that predict simple quark "popping" of equal probability for quarks and antiquarks give predictions for the ratio of meson production to baryon production of between 2 [23] and $6[24,25]$.

An interesting feature of the relative fractions to produce a quark from the vacuum is that it is sensitive to the quark masses. One can therefore use measured values of $\gamma_{s} / \gamma_{u}$ and $\gamma_{s} / \gamma_{d}$ to determine the mass of the strange quark relative to the lighter quarks $[26]$.

The $\alpha_{j}$ values in Equation 1.9 have typically been ignored in most studies, in which all the $\alpha_{j}$ values are taken to be zero with the exception of the lowest lying mesons, the ${ }^{1}{ }^{1} S_{0}$ states. When this is done, Equation 1.9 is greatly simplified and the only remaining unknowns in Equation 1.9 are the branching fractions of the hadrons and the $\gamma_{i}$ values. Thus, the ratio of branching fractions of hadrons can be measured in a way that only depends on the ratio of $\gamma_{i}$ values. With the additional requirement that the hadrons decay to states with the same valence quarks, one can rewrite a ratio of Equation 1.9 as

$$
\frac{\mathcal{B}\left(B_{1} \rightarrow J / \psi X_{1}\right) \cdot \gamma_{1}}{\mathcal{B}\left(B_{2} \rightarrow J / \psi X_{2}\right) \cdot \gamma_{2}}=\frac{N\left(B_{1} \rightarrow J / \psi X_{1}\right) \cdot \epsilon_{2}\left(J / \psi, X_{2}, \mathcal{B}_{2}\right)}{N\left(B_{2} \rightarrow J / \psi X_{2}\right) \cdot \epsilon_{1}\left(J / \psi, X_{1}, \mathcal{B}_{1}\right)}
$$

It is with Equation 1.14 that the ratios of branching fractions times fragmentation fractions are measured. For this analysis, Equation 1.14 is used in conjunction with the five decays that are listed in Equation 1.15 (including the charge conjugate decays that are always implied): 


$$
\begin{array}{ll}
1 & B^{+} \rightarrow J / \psi K^{+} \\
2 & B^{+} \rightarrow J / \psi K^{*}(892)^{+} \\
3 & B^{0} \rightarrow J / \psi K^{0} \\
4 & B^{0} \rightarrow J / \psi K^{*}(892)^{0} \\
5 & B_{s}^{0} \rightarrow J / \psi \phi(1020)
\end{array}
$$

With these decays, Equation 1.14 can be rewritten and presented as a $5 \times 5$ matrix, which is shown below with the indices corresponding to the order of the decays given above:

$$
\begin{array}{lllll}
\frac{\mathcal{B}(1)}{\mathcal{B}(1)} \frac{\gamma_{1}}{\gamma_{1}} & \frac{\mathcal{B}(1)}{\mathcal{B}(2)} \frac{\gamma_{1}}{\gamma_{2}} & \frac{\mathcal{B}(1)}{\mathcal{B}(3)} \frac{\gamma_{1}}{\gamma_{3}} & \frac{\mathcal{B}(1)}{\mathcal{B}(4)} \frac{\gamma_{1}}{\gamma_{4}} & \frac{\mathcal{B}(1)}{\mathcal{B}(5)} \frac{\gamma_{1}}{\gamma_{5}} \\
\frac{\mathcal{B}(2)}{\mathcal{B}(1)} \frac{\gamma_{2}}{\gamma_{1}} & \frac{\mathcal{B}(2)}{\mathcal{B}(2)} \frac{\gamma_{2}}{\gamma_{2}} & \frac{\mathcal{B}(2)}{\mathcal{B}(3)} \frac{\gamma_{2}}{\gamma_{3}} & \frac{\mathcal{B}(2)}{\mathcal{B}(4)} \frac{\gamma_{2}}{\gamma_{4}} & \frac{\mathcal{B}(2)}{\mathcal{B}(5)} \frac{\gamma_{2}}{\gamma_{5}} \\
\frac{\mathcal{B}(3)}{\mathcal{B}(1)} \frac{\gamma_{3}}{\gamma_{1}} & \frac{\mathcal{B}(3)}{\mathcal{B}(2)} \frac{\gamma_{3}}{\gamma_{2}} & \frac{\mathcal{B}(3)}{\mathcal{B}(3)} \frac{\gamma_{3}}{\gamma_{3}} & \frac{\mathcal{B}(3)}{\mathcal{B}(4)} \frac{\gamma_{3}}{\gamma_{4}} & \frac{\mathcal{B}(3)}{\mathcal{B}(5)} \frac{\gamma_{3}}{\gamma_{5}} \\
\frac{\mathcal{B}(4)}{\mathcal{B}(1)} \frac{\gamma_{4}}{\gamma_{1}} & \frac{\mathcal{B}(4)}{\mathcal{B}(2) \frac{\gamma_{4}}{\gamma_{2}}} & \frac{\mathcal{B}(4)}{\mathcal{B}(3)} \frac{\gamma_{4}}{\gamma_{3}} & \frac{\mathcal{B}(4)}{\mathcal{B}(4)} \frac{\gamma_{4}}{\gamma_{4}} & \frac{\mathcal{B}(4)}{\mathcal{B}(5)} \frac{\gamma_{4}}{\gamma_{5}} \\
\frac{\mathcal{B}(5)}{\mathcal{B}(1)} \frac{\gamma_{5}}{\gamma_{1}} & \frac{\mathcal{B}(5)}{\mathcal{B}(2)} \frac{\gamma_{5}}{\gamma_{2}} & \frac{\mathcal{B}(5)}{\mathcal{B}(3)} \gamma_{5} & \frac{\mathcal{B}(5)}{\gamma_{3}} \gamma_{5} & \frac{\mathcal{B}(5)}{\mathcal{B}(4)} \gamma_{5} \\
\gamma_{4} & \mathcal{B}(5) \gamma_{5}
\end{array}
$$

Given the matrix elements in Equation 1.16, one can then determine the ratios of branching fractions for decays of the same bottom meson since the $\gamma_{i}$ values will cancel out. Alternatively, one can determine the ratio of branching fractions of different hadrons by making assumptions about the ratio of the $\gamma_{i}$ values.

An added benefit of using the matrix given by Equation 1.16 to calculate branching fractions is that many efficiencies and systematic uncertainties cancel out in the ratios because the five decays are topologically similar in that all five bottom mesons have very similar masses and all of the five decays involve a $J / \psi$ meson and a meson that contains a strange quark. This is best illustrated by the lowest order Feynman diagrams, which are identical with the exception of the spectator quarks. The lowest order Feynman diagram for the generic $B_{q} \rightarrow J / \psi X_{q}$ decay is shown in Figure 1.4.

One can also determine the ratio of the $\gamma_{i}$ values by using phenomenological predictions for the ratios of branching fractions. Since the non-spectator part of the Feynman diagram is the same for the five decays listed above, many unknown parameters used in theoretical models will also cancel out when taking ratios of branching 


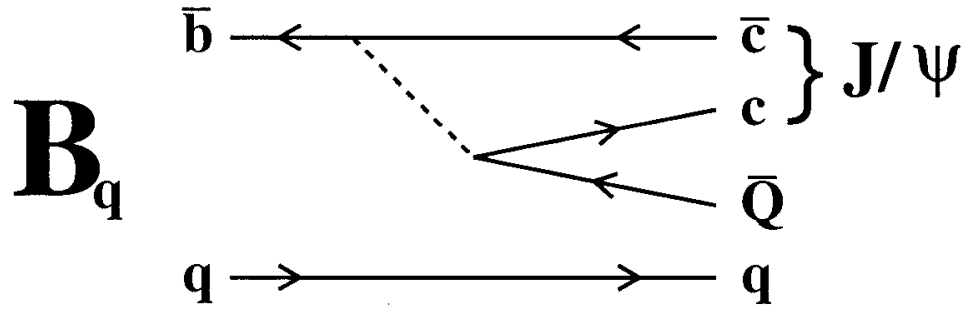

Figure 1.4: The lowest order Feynman diagram for a two body $B$ meson decay into states involving a $J / \psi$ meson.

fractions. One can then use the theoretical models to obtain the ratios of fragmentation fractions for $b$ quarks to hadronize to the various $B$ mesons. Some commonly used theoretical models will be discussed in the next section.

Although the matrix elements given in Equation 1.16 can be used to determine either the ratios of branching fractions or the ratios of fragmentation fractions, one should keep in mind all the assumptions that one has to make to be able to obtain the relationship given in Equation 1.14 from that given in Equation 1.9. The assumptions are summarized below:

1. The correct shape of the $\bar{p} p \rightarrow b X$ production cross section is known.

2. The $\alpha_{j}$ values (probability to produce mesons in a given radial-spin-parityorbital angular momentum state) are assumed to be zero except for the lowest lying states.

3. Bottom hadrons do not decay to other bottom hadrons in which the spectator quarks are changed.

The first assumption results in a relatively small uncertainty when taking ratios, because the theoretical model for the bottom quark production cross section from proton-antiproton collisions at $\sqrt{s}=1.8 \mathrm{TeV}$ has approximately the correct shape as that of the data [15], which is illustrated in Figure 1.3. The theoretical model consists of the next-to-leading order QCD calculation $[11,12,16]$ with the renormalization scale $\mu_{0}=\sqrt{m_{b}^{2}+P_{T}^{2}}$, where the $b$ quark mass, $m_{b}$, is set to $4.75 \mathrm{GeV} / c^{2}$. The model also uses the $\mathrm{MRSD}_{0}$ parton distribution function [14] and the Peterson parameterization $[17,18]$ for the fragmentation functions. The Peterson fragmentation parameter 
$(\epsilon)$ is set to 0.006 . A systematic uncertainty in the ratios of branching fractions times fragmentation fractions due to the shape is included to account for these effects.

The third assumption is valid if the second assumption is correct (only $B^{+}, B^{0}$, $B_{s}^{0}$ and $B_{c}^{+}$mesons are produced). Because the mass difference between the $B^{+}, B^{0}$ and $B_{s}^{0}$ is less than the pion mass, one bottom meson state cannot decay to another one with different flavored quarks. This is not true for the $B_{c}^{+}$meson, which can decay to lower mass bottom meson states. However, because the $B_{c}^{+}$production rate is predicted to be very small as given in Equation 1.13, it will not significantly contribute to the observed decays.

The second assumption is the weakest. In principle, the production rate of the higher $N^{2 S+1} L_{J}$ states could be large, though states with $N>1$ should be harder to produce. For example, the $\psi^{\prime}\left(2^{3} S_{1}\right)$ direct production rate is around two orders of magnitude smaller than the $J / \psi\left(1^{3} S_{1}\right)$ production rate at $\sqrt{s}=1.8 \mathrm{TeV}$ [27], but the production rates for the different radial $\Upsilon$ states do not vary as much [28]. Although the production rates of the $N>1$ states are expected to be relatively small, the production rates for states with $N=1, L>0, S>0$ and $J>0$ are not. For example, the quark model $1^{1} S_{0}$ and $1^{3} S_{1}$ states relative production rates can be either 1:1 or 1:3 depending on whether state counting or spin counting is applicable (for spin counting, the production rate is proportional to $2 J+1$ ). Thus the production rate of the $B^{*+}$ mesons can be, in principle, three times that of the $B^{+}$meson. However, because the mass differences between the $1^{1} S_{0}$ and the $1^{3} S_{1}$ bottom meson states are around $45 \mathrm{MeV} / c^{2}$, the $1^{3} S_{1}$ states can only decay with the emission of a soft photon to the same flavored $1^{1} S_{0}$ bottom meson states. Because of the small mass difference, the differential production cross sections for the $1^{1} S_{0}$ and $1^{3} S_{1}$ states will also be very similar [29]. This implies that the detection efficiencies for the two states will be practically the same and so this violation of the second assumption has little practical effect.

The second assumption is expected to fail when one considers orbitally excited hadrons because of the larger expected mass differences. Orbitally excited hadrons $(L>0)$ can decay strongly to hadrons with different flavored spectator quarks. This will cause an imbalance in the number of flavored bottom hadrons detected, with the lighter flavored hadrons being more abundant. In addition, these hadrons could have a differential production cross section that differs from theoretical expectations. This imbalance of hadron production has already been noticed in the charm system 
where the orbitally excited $c \bar{s}$ states have been found to strongly decay to $c \bar{u}$ and $c \bar{d}$ states but not vice versa [7]. The production rate of orbitally excited hadronic states have also been shown to be noticeable in bottom quark fragmentation. The relative production rates of the orbitally excited $B^{+}$and $B^{0}$ mesons to the ground states have already been measured to be around $30 \%$ at the $\mathrm{Z}$ mass region $[30,31]$. In order to obtain the $\gamma_{i}$ values one must therefore take into account this effect.

If the efficiencies to detect the ground state bottom hadrons that result from decays of excited states are approximately equal to the efficiencies to detect the same bottom hadrons that were produced directly, one can then approximate $\gamma_{i} \sim f_{i}$. Nevertheless, it should be noted that the assumption still fails if there is an imbalance of produced hadrons through decays of exited states with different flavored spectator quarks.

Given the observed ratios of detected decays with the assumptions mentioned above, the ratios of branching fractions times fragmentation fractions can be calculated. These calculations are presented in Chapter 6.

With the knowledge of the ratios of branching fractions given in Equation 1.16 one can then factor out the ratios of branching fractions and compare the remaining $\gamma$ ratios with the theoretical predictions given in Equation 1.13.

\subsection{Non-Leptonic decays of Bottom Mesons}

As mentioned in the previous section, one can measure the relative probabilities to produce quarks and antiquarks out of the vacuum if one knows the ratios of branching fractions for the bottom meson states being studied. The branching fractions of two body non-leptonic decays of bottom mesons are given by the partial widths for each decay mode times the lifetimes of the bottom mesons.

The partial width of a two body non-leptonic decay of a hadron cannot be unambiguously calculated in the standard model due to the complex interplay between the weak and strong forces at various distances. Fortunately, if one makes a few approximations and assumptions one can reduce the problem to one that can be solved with the currently available theoretical tools.

One begins with the assumption that the nonleptonic decays are governed by the standard model of electroweak interactions $[2,3,4]$ in which the Hamiltonian is of the type given by 


$$
H(x)=\frac{g}{2 \sqrt{2}} J_{\mu}^{+}(x) \cdot W_{\mu}^{-}(x)+h . c .,
$$

where $x$ is the momentum transfer, $g$ is the weak coupling constant, $W_{\mu}^{-}$is the charged $W$ boson field, and $J_{\mu}^{+}$is the charged weak current given by

$$
J_{\mu}^{+}(x)=(\bar{u}(x), \bar{c}(x), \bar{t}(x)) \gamma_{\mu}\left(1-\gamma^{5}\right)\left(\begin{array}{c}
d^{\prime} \\
s^{\prime} \\
b^{\prime}
\end{array}\right) .
$$

The primes on the quark fields arise because the quark mass eigenstates are not the same as the weak eigenstates but are related by the transformation

$$
\left(\begin{array}{c}
d^{\prime} \\
s^{\prime} \\
b^{\prime}
\end{array}\right)=\left(\begin{array}{lll}
V_{u d} & V_{u s} & V_{u b} \\
V_{c d} & V_{c s} & V_{c b} \\
V_{t d} & V_{t s} & V_{t b}
\end{array}\right)\left(\begin{array}{c}
d \\
s \\
b
\end{array}\right)
$$

The transformation matrix is known as the Cabibbo-Kobayashi-Maskawa (CKM) mixing matrix [32,33], and the current limits [7] on the magnitude of the matrix elements are

$$
\left(\begin{array}{ccc}
0.9747-0.9759 & 0.218-0.224 & 0.002-0.005 \\
0.218-0.224 & 0.9738-0.9752 & 0.032-0.048 \\
0.004-0.015 & 0.030-0.048 & 0.9988-0.9995
\end{array}\right),
$$

where the numbers quoted are limits at the $90 \%$ confidence level and are based on unitarity and the assumption that there are only three quark generations. The transformation for the charge $2 / 3$ quarks is taken to be the identity matrix by convention.

Ignoring strong interactions, the lowest-order weak current-current interaction at zero momentum transfer is given by an effective Hamiltonian

$$
H_{e f f}(0)=\frac{G_{F}}{\sqrt{2}} J_{\mu}^{+}(0) J_{\mu}^{-}(0)+h . c .,
$$

were $G_{F}$ is the Fermi coupling constant.

Strong interaction effects are taken into account by making additional assumptions. The first is that all long distance effects, which include soft gluon radiation, final state interactions and the creation of quark-antiquark pairs from the vacuum, can be absorbed into the initial and final state wavefunctions. The short distance effects, which are dominated by the exchange of hard gluons, can be calculated within QCD. Following references [34, 35], the effective Hamiltonian for bottom meson decays with $O\left(\alpha_{s}\right)$ terms can be rewritten as 


$$
\begin{aligned}
H_{e f f}= & \frac{G_{F}}{\sqrt{2}} V_{c b}\left[C_{1}(\mu)\left((\bar{c} b)\left(\bar{d}^{\prime} u\right)+(\bar{c} b)\left(\bar{s}^{\prime} c\right)\right)+C_{2}(\mu)\left(\left(\bar{d}^{\prime} b\right)(\bar{c} u)+\left(\bar{s}^{\prime} b\right)(\bar{c} c)\right)\right]+ \\
& \frac{G_{F}}{\sqrt{2}} V_{u b}\left[C_{1}(\mu)\left((\bar{u} b)\left(\bar{d}^{\prime} u\right)+(\bar{u} b)\left(\bar{s}^{\prime} c\right)\right)+C_{2}(\mu)\left(\left(\bar{d}^{\prime} b\right)(\bar{u} u)+\left(\bar{s}^{\prime} b\right)(\bar{u} c)\right)\right]+h . c .
\end{aligned}
$$

were $C_{1}$ and $C_{2}$ are Wilson coefficients that are calculated from QCD at the scale $\mu$, which is usually taken to be the mass of the heavy quark. The current terms $\left(\bar{q}_{1} q_{2}\right)$ in Equation 1.22 are given by

$$
\left(\bar{q}_{1} q_{2}\right)=\sum_{i} \bar{q}_{1}^{i} \gamma_{\mu}\left(1-\gamma^{5}\right) q_{2}^{i}
$$

with the summation index $i$ running over the three colors.

With the above assumptions, the partial width $(\Gamma)$ of a given decay is proportional to the square of the matrix element with the Hamiltonian taken from Equation 1.22. For example, the partial width of the generic $B_{q} \rightarrow J / \psi X_{q}$ decay is given by

$$
d \Gamma\left(B_{q} \rightarrow J / \psi X_{q}\right) \quad \propto \quad\left|<J / \psi, X_{q}\right| H_{e f f}\left|B_{q}>\right|^{2} .
$$

The last ansatz that is needed to evaluate the above partial width is the factorization ansatz. In the popular Bauer, Stech and Wirbel $[36,37]$ factorization ansatz, it is assumed that the currents in Equation 1.23 are hadronic currents and that the two hadronic currents can be separated out from the matrix element between the initial and final states. In this scheme, the Wilson coefficients $\left(C_{1}, C_{2}\right)$ are replaced with free parameters $\left(a_{1}, a_{2}\right)$ that can be determined from fits to data. The new parameters are related to the Wilson coefficients through the relationship

$$
\begin{aligned}
& a_{1}(\mu)=C_{1}(\mu)+\xi C_{2}(\mu) \\
& a_{2}(\mu)=C_{2}(\mu)+\xi C_{1}(\mu)
\end{aligned}
$$

where $\xi \sim 1 / N_{\text {color }}$, with $N_{\text {color }}$ being the number of colors. The parameter $a_{1}$ is assigned to the effective neutral hadronic current and $a_{2}$ is assigned to the effective charged hadronic current.

For the decay $B_{q} \rightarrow J / \psi X_{q}$ with $B_{q} \equiv \bar{q} b$ and $X_{q} \equiv \bar{q} s$, the partial width from Equation 1.24 becomes

$$
\begin{aligned}
d \Gamma\left(B_{q} \rightarrow J / \psi X_{q}\right) & \propto \frac{G_{F}^{2}}{2} \cdot a_{2}^{2}\left(m_{b}\right) \cdot\left|V_{c b} V_{c s}^{*}\right|^{2}\left|<J / \psi, X_{q}\right|(\bar{s} b)(\bar{c} c)\left|B_{q}>\right|^{2} \\
& \propto \frac{G_{F}^{2}}{2} \cdot a_{2}^{2}\left(m_{b}\right) \cdot\left|V_{c b} V_{c s}^{*}\right|^{2}|<J / \psi|(\bar{c} c)|0>|^{2}\left|<X_{q}\right|(\bar{s} b)\left|B_{q}>\right|^{2} .
\end{aligned}
$$


The one particle matrix element $\langle J / \psi|(\bar{c} c)| 0\rangle$ is given by the momentum and the decay constant of the particle. Thus, the problem of determining the partial width reduces to the problem of determining the matrix element $\left\langle X_{q}|(\bar{s} b)| B_{q}>\right.$. There are many different types of phenomenological models that predict the values for $\left\langle X_{q}|(\bar{s} b)| B_{q}>\right.$. Two models that predict the branching fractions of $B^{+}, B^{0}$ and $B_{s}^{0}$ mesons are given in Table 1.5. The two models differ in many details, such as the magnitude and shape of the form factors for $B$ meson decays and the experimental constraints employed in the calculations.

The model that gives the predictions shown in the first column of Table 1.5 uses form factors that are normalized to $D$ meson semileptonic decay data and are assumed to be consistent with simple pole dominance. In addition, the parameters $a_{1}$ and $a_{2}$ are treated as free parameters.

The model that gives the predictions shown in the second column of Table 1.5 uses form factors calculated with relativistic quark wavefunctions and are assumed to be consistent with simple pole dominance. The wavefunctions are taken to be solutions of a harmonic oscillator potential and the QCD parameters are not treated as free parameters.

One important thing to note from Equation 1.26 is that many theoretical parameters will cancel when one calculates ratios of branching fractions for decays that are described by the generic $B_{q} \rightarrow J / \psi X_{q}$ decay. Thus one can use the theoretical ratios of branching fractions in conjunction with the experimentally measured ratios of branching fractions times fragmentation fractions to obtain the ratios of fragmentation fractions of the $b$ quarks to the various bottom hadrons with only a few assumptions.

Alternatively, one can test the factorization ansatz as well as the different theoretical predictions for the matrix element $<X_{q}|(\bar{s} b)| B_{q}>$ through comparisons with measured ratios of branching fractions. Since what is measured is a product of fragmentation fractions times branching fractions, one is limited to a comparison of ratios of branching fractions of same flavored mesons. However, comparisons with theoretical predictions can also be made for ratios that involved different flavored mesons if one knows the corresponding ratios of fragmentation fractions. 


\begin{tabular}{|l|c|c|}
\hline \hline Decay & Branching Fraction (\%) & Branching Fraction (\%) \\
\hline \hline$B^{+} \rightarrow J / \psi K^{+}$ & $0.11 \pm 0.06$ & \\
\hline$B^{+} \rightarrow J / \psi K^{*}(892)^{+}$ & $0.16 \pm 0.05$ & 0.242 \\
\hline$B^{0} \rightarrow J / \psi K^{0}$ & $0.11 \pm 0.06$ & \\
\hline$B^{0} \rightarrow J / \psi K^{*}(892)^{0}$ & $0.16 \pm 0.05$ & 0.241 \\
\hline$B_{s}^{0} \rightarrow J / \psi \phi(1020)$ & $0.14 \pm 0.04$ & $0.273-0.313$ \\
\hline \hline
\end{tabular}

Table 1.5: Branching fraction predictions based on the factorization ansatz. The first column contains the predictions from $[38,39]$ with all of the bottom mesons having the same lifetime of 1.4 ps. The second column contains the predictions from [40,41], with all of the bottom mesons having the same lifetime of $1.3 \mathrm{ps}$. 


\section{Chapter 2}

\section{The Experimental Apparatus}

The experimental apparatus that was used for this analysis is situated at the Fermi National Accelerator Laboratory located at Batavia, Illinois, U.S.A.

The experimental apparatus can be broken down into two logical groups that consist of the accelerator and the detector. The accelerator, which is commonly called the Tevatron, is used to accelerate protons and antiprotons up to energies of $900 \mathrm{GeV}$. The protons and antiprotons then collide to produce new particle species such as the various bottom mesons that are examined in this analysis.

The detector, which is called the Collider Detector at Fermilab (CDF), is used to measure various properties of some of the particles originating from the protonantiproton collision. For a typical proton-antiproton collision, the CDF detector collects approximately 165 thousand bytes of information.

\subsection{The Tevatron Accelerator}

The protons and antiprotons are accelerated to the maximum achievable energy (900 $\mathrm{GeV}$ stable beam, $950 \mathrm{GeV}$ unstable beam) in various stages. In total, six accelerators are used for the antiproton acceleration, four of which are also used for the proton acceleration.

The proton beam starts as a stream of hydrogen gas (an ion source $\left(\mathrm{H}^{-}\right)$in an intense electric field). The ions are then directed into a $30 \mathrm{~cm}$ accelerating (highvoltage) column which is maintained at 0.75 MegaVolts by a Cockcroft-Walton power supply. The emerging ions from this preaccelerator have an energy of $0.75 \mathrm{MeV}$ and the typical current at this stage is $50 \mathrm{~mA}$ [42]. The resulting beam is then received by a 175 meter linear accelerator (Linac). The Linac accelerates the ions in pulses with 


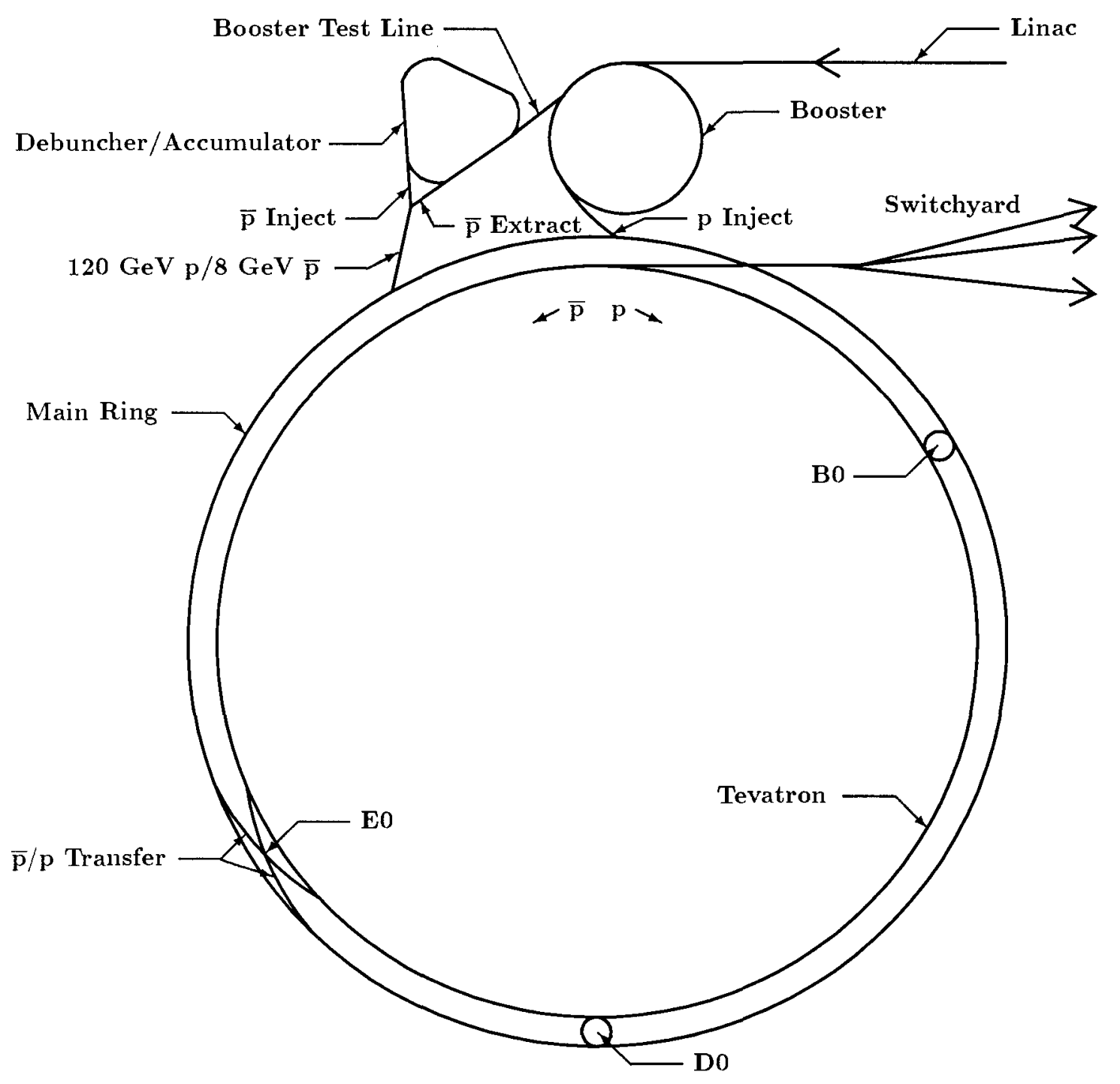

Figure 2.1: Schematic of the Linac, Booster, Main Ring, Debuncher/Accumulator and Tevatron (not drawn to scale). 


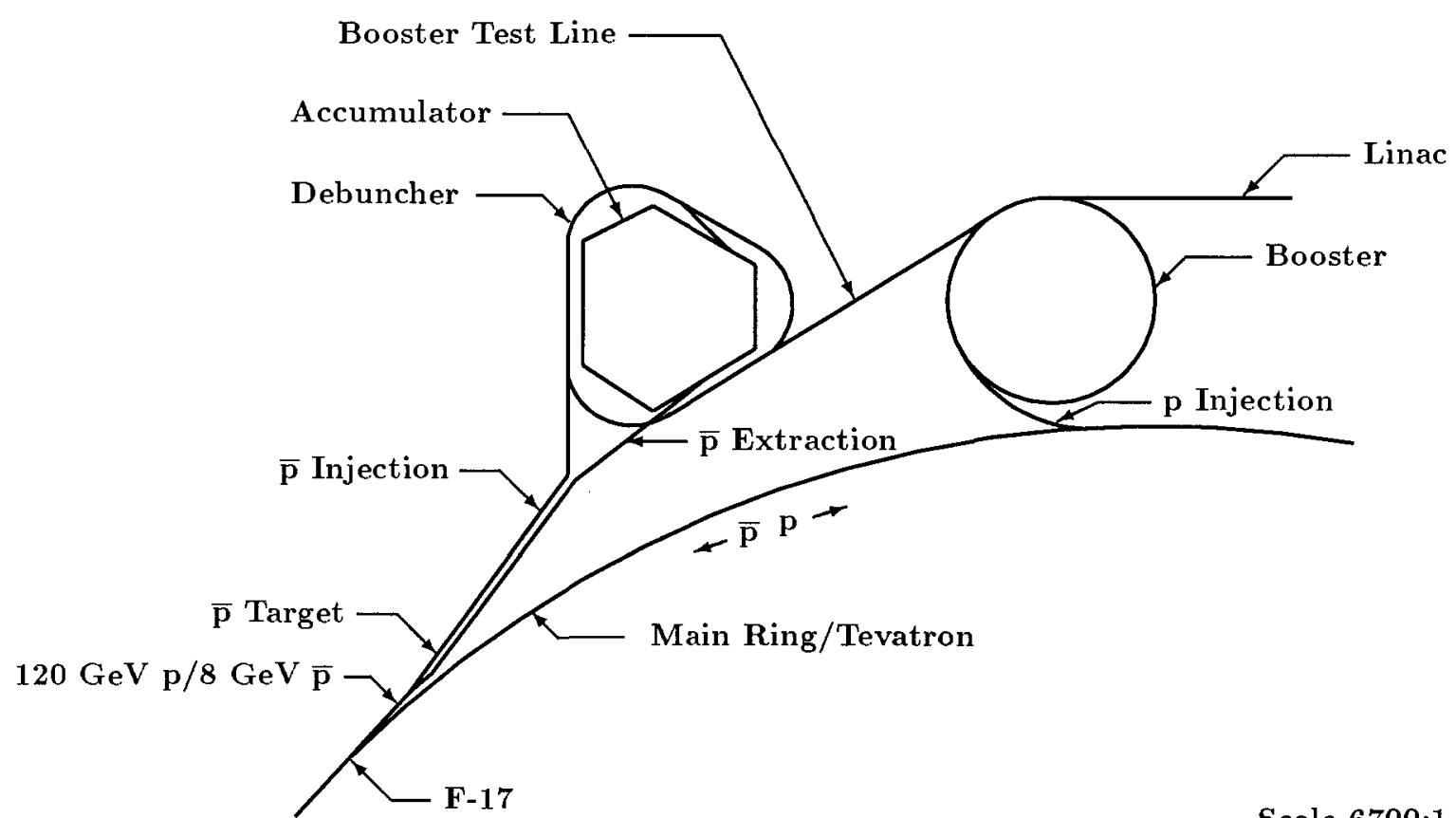

Scale 6700:1

Figure 2.2: Schematic of the Linac, Booster, Main Ring, Debuncher, Accumulator and Tevatron (drawn to scale).

a $15 \mathrm{~Hz}$ pulsing frequency (each pulse lasts about $30 \mu \mathrm{s}$ ). The resulting ion energy after the Linac stage is $200 \mathrm{MeV}$ (this stage was recently upgraded [43]). The ions are then targeted on a thin carbon foil that is used to strip the two electrons from the hydrogen ion. The next stage in the acceleration involves a series of 9610 -foot long magnets (Booster) arranged in a circle with a 75 meter radius. The series of magnets causes the protons to follow a circular orbit by increasing the magnetic field strength as the proton energy increases. The magnetic fields vary from $0.05 \mathrm{~T}$ at injection to $0.67 \mathrm{~T}$ at extraction. The protons circle 16 thousand times in $33 \mu$ s before they reach the maximum energy of $8 \mathrm{GeV}$ [44].

For antiproton production, protons are injected into the main ring accelerator at point A0 (p Inject in Figure 2.1). The main ring accelerator consists of more than a thousand magnets attached together in a circular pattern with a $6.28 \mathrm{~km}$ circumference. There are 77220 -ft long bending dipole magnets, 1927 -ft long quadrupole focusing magnets and 48 -ft quadrupoles for beam adjustment. The main ring accelerates the protons to $120 \mathrm{GeV}$. The protons are then extracted at point F-17 in Figure 2.2 and directed towards a $5 \mathrm{~cm}$ tungsten target where they collide with the 
atoms in the tungsten target and produce a cornucopia of particles. All of the negatively charged particles with a momentum of $8.9 \pm 0.3 \mathrm{GeV} / c$ are collected and transported to the debuncher synchrotron[45]. After a few cycles in the debuncher (a few milliseconds) only the antiprotons survive. In addition to removing the other particles species, the debuncher also stochastically cools the beam (reduces the fractional momentum spread of the antiprotons) [46]. After a couple of seconds of cooling, the antiprotons are injected into the accumulator where the antiprotons are stored (stacked) and further cooled for later injection into the Tevatron via the main ring accelerator. This cycle continues until there is a sufficient number of antiprotons available in the stack (accumulator) for high luminosity collisions.

When the amount of antiprotons in the stack reaches a suitable value (typically $5 \times 10^{11}$ antiprotons), the protons are no longer directed towards the tungsten target. Instead, a bunch of protons from the booster is accelerated to $150 \mathrm{GeV}$ and then injected into the Tevatron. This is done six times and results in six bunches circulating in the Tevatron at the same time. The Tevatron consists of over a thousand superconducting magnets arranged in a similar pattern to that of the main ring magnets (actually located below the main ring magnets). The Tevatron is made up of 772 dipole bending magnets, 224 focusing quadrupole magnets and 720 small correction and adjustment magnets. The maximum magnetic field strength is 4.4 Tesla at a temperature of 4.7 degrees Kelvin [47].

When all of the six proton bunches are injected into the Tevatron, the booster is turned off, and the antiprotons with a typical energy of $8 \mathrm{GeV}$ are extracted from the accumulator and injected into the main ring in the opposite direction to the protons. The antiproton bunch is then accelerated to $150 \mathrm{GeV}$ and inserted into the Tevatron in the opposite direction to the protons. This is repeated five more times resulting in six proton and six antiproton bunches circulating inside the Tevatron at the same time. Finally, the 12 bunches are accelerated to $900 \mathrm{GeV}$, resulting in $\bar{p} p$ collisions at a center-of-mass energy $(\sqrt{s})$ of $1.8 \mathrm{TeV}$. Once colliding beams are in place, the booster and main ring are turned on again for antiproton production and stacking.

\subsection{The Collider Detector at Fermilab}

The Collider Detector at Fermilab (CDF) is a magnetic detector used to study $\bar{p} p$ collisions at the B0 interaction point at the Tevatron accelerator. The detector consists 


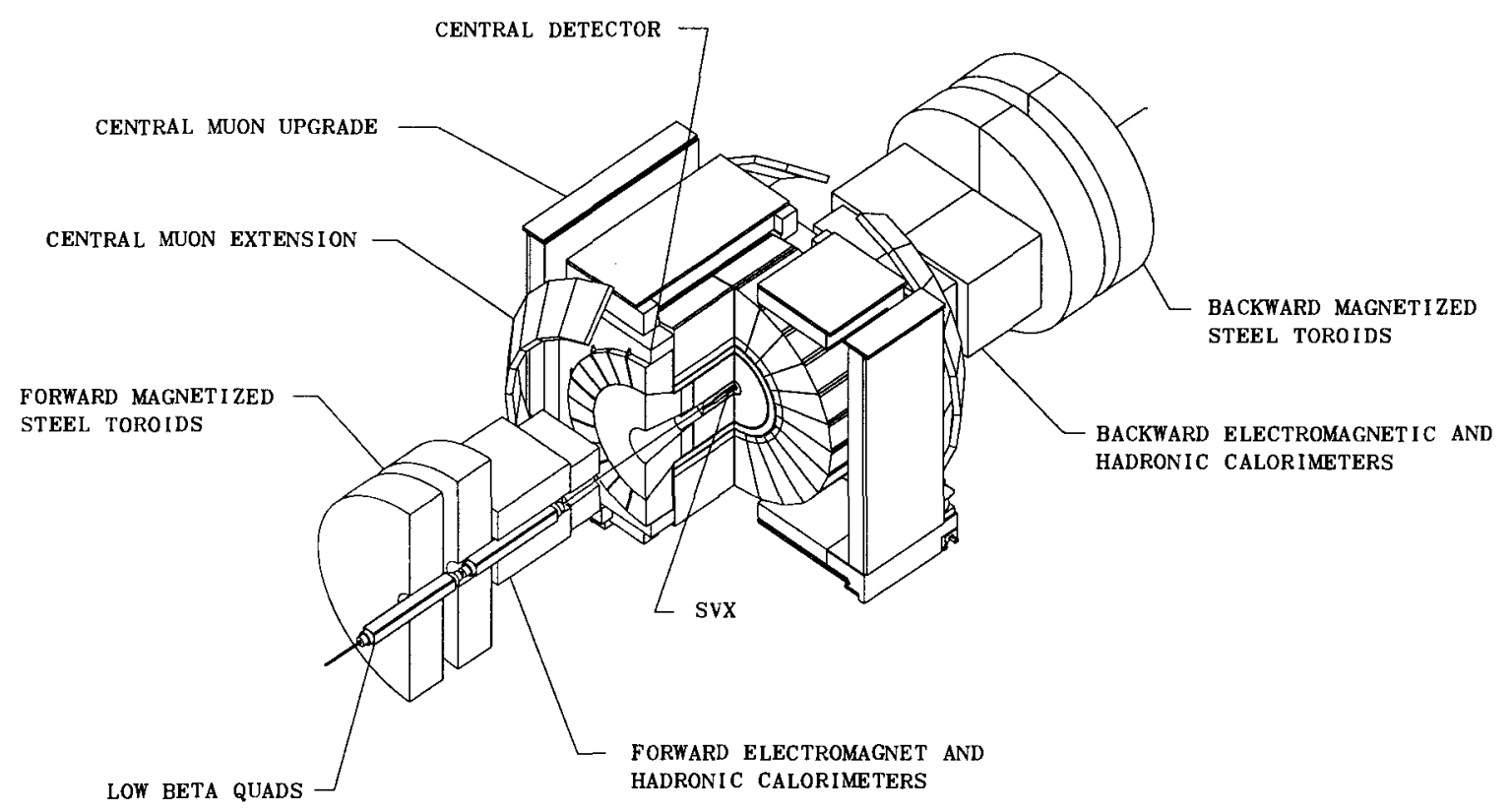

Figure 2.3: Isometric display of the CDF detector with a cut-a-way view of one quadrant. The central detector (region) consists of the material between the central muon extension chambers.

of three regions that are both azimuthal and forward-backward symmetric. One of the three regions is the central detector, which consists of tracking systems, electromagnetic shower counters, electromagnetic and hadronic calorimeters, muon detection chambers and a solenoid magnet. The forward-backward detector regions consists of time-of-flight counters, electromagnetic shower counters, hadron calorimeters and muon toroidal spectrometers. The three regions can be seen in Figure 2.3.

The central detector consists of 14 subdetectors occupying a volume of $12 \mathrm{~m} \times 12$ $\mathrm{m} \times 12 \mathrm{~m}$. Starting from the $\bar{p} p$ collision point, there is a 1.5 in diameter, 20 mil thick beryllium pipe. Outside the beryllium pipe lies a silicon microstrip vertex detector. The SVX detector consists of two cylindrical modules each with four layers of silicon, which are located at distances from $3.0 \mathrm{~cm}$ to $7.9 \mathrm{~cm}$ away from the beam line and $\pm 25.55 \mathrm{~cm}$ along the beam line starting at $z= \pm 0.5 \mathrm{~cm}$. Given that the standard deviation of the distribution of $\bar{p} p$ collisions along the beam line is approximately 30 $\mathrm{cm}, 60 \%$ of the collisions occur within the fiducial volume of the SVX detector. The primary purpose of the SVX is to provide precision track reconstruction in the plane 


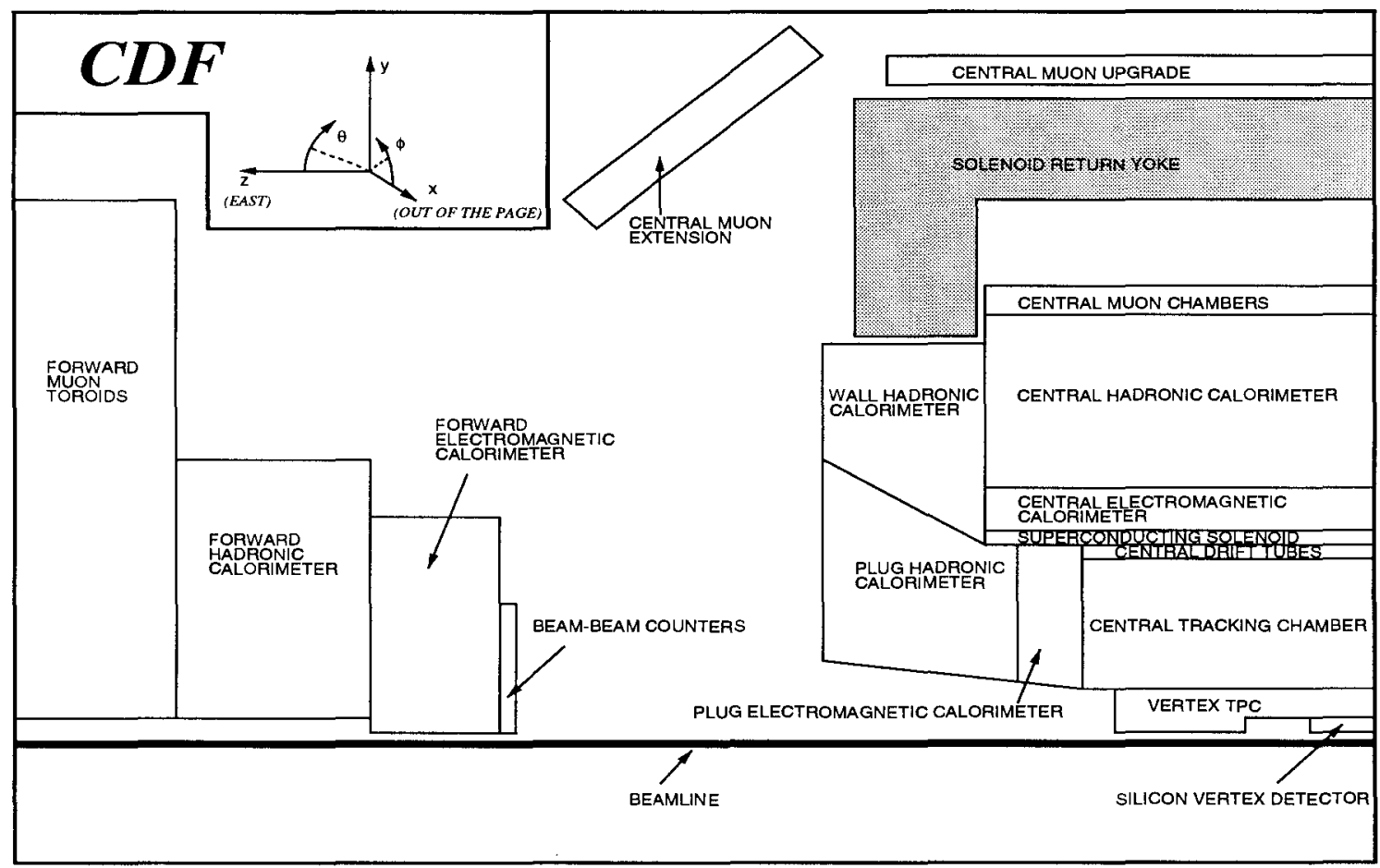

Figure 2.4: Longitudinal profile of one quadrant of the CDF detector. All of the chambers except the CMX chambers span the entire region in $\phi$. The CMX quadrant spans the $\phi$ interval between $-45 /+135$ degrees to $+75 /+255$ degrees.

transverse to the beam. The impact parameter (distance of closest approach to the beam line) resolution for a high momentum track measured by the SVX detector is $17 \mu \mathrm{m}$.

The Vertex (VTX) Drift Chamber surrounds the SVX detector, as shown in Figure 2.4. The VTX detector provides tracking information in the $r-z$ plane up to a distance of $132 \mathrm{~cm}$ along the beam line in either direction away from the center and up to a radius of $22 \mathrm{~cm}$. The reconstructed tracks from the VTX chamber are also used to calculate the longitudinal position of the $\bar{p} p$ collision point with a resolution of approximately $1 \mathrm{~mm}$.

Both the SVX and VTX chambers are mounted inside the Central Tracking Chamber (CTC), which is within a $1.4 \mathrm{~T}$ axial magnetic field produced by a superconducting solenoid. The CTC is a $3.2 \mathrm{~m}$ long multi-wire drift chamber with inner and outer radii of $31 \mathrm{~cm}$ and $132 \mathrm{~cm}$. It consists of 6156 sense wires arranged in 84 concentric cylindrical layers organized into 9 superlayers as shown in Figure 2.5. Sixty layers have wires that are parallel to the beam direction (axial wires). These wires provide 


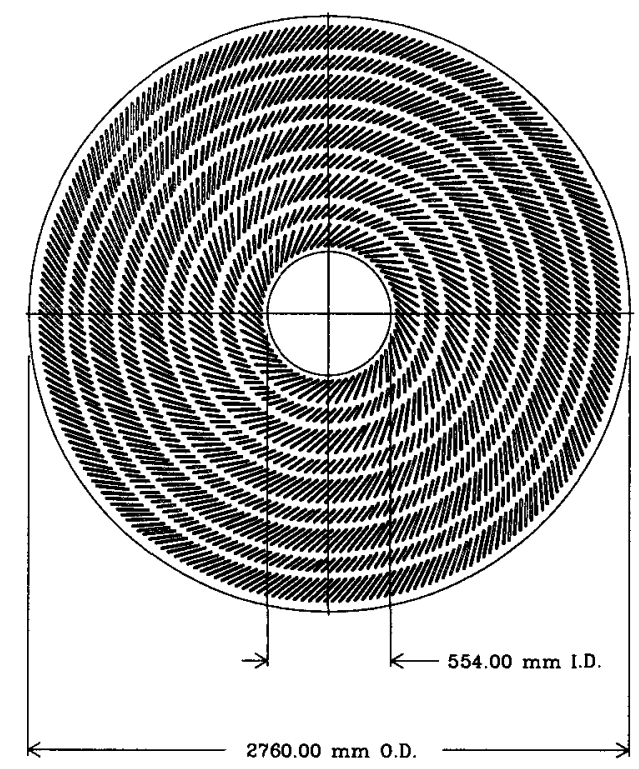

Figure 2.5: Beam line view of the central tracking chamber. Each box represents a grouping of axial or stereo wires. Five rings of the larger boxes (axial superlayers) consists of axial wires with 12 radial sense wires per box. Four rings of the smaller boxes (stereo superlayers) consists of stereo wires with 6 radial sense wires per box.

tracking in the $r-\phi$ plane. The remaining layers are tilted by 3 degrees with respect to the beam line, which together with the other sixty layers provide tracking in the $r-z$ plane (stereo layers). Particle positions measured by the CTC, VTX and if available, SVX are fit to the helical trajectory that one expects for a charged track traversing an axial magnetic field. The helical trajectory can be fully described by the following five parameters: radius of curvature, initial theta angle $(\theta)$ or $\eta$ (pseudorapidity $\eta=-\ln [\tan (\theta / 2)])$, initial $\phi$ angle, initial $z$ value and the transverse distance of closest approach to the beam line (impact parameter). These five parameters are then used to determine the initial momentum vector and vertex position of the particle that traversed the chambers. An example of fitted charged particle trajectories are illustrated in Figure 2.6.

The transverse momentum (component perpendicular to the beam line) resolution with the combined information from the three tracking chambers is $\delta P_{T}=$ $\left[\left(0.009 P_{T}\right)^{2}+(0.0066)^{2}\right]^{\frac{1}{2}}$, where $P_{T}$ is in units of $\mathrm{GeV} / c$. The $z$ vertex resolution is approximately $1 \mathrm{~mm}$, while the impact parameter resolution is approximately $50 \mu \mathrm{m}$ for particles with a transverse momentum of $1 \mathrm{GeV} / c$. The typical $\phi$ angle resolution for particles with a transverse momentum of $1 \mathrm{GeV} / c$ is approximately 0.025 degrees. 


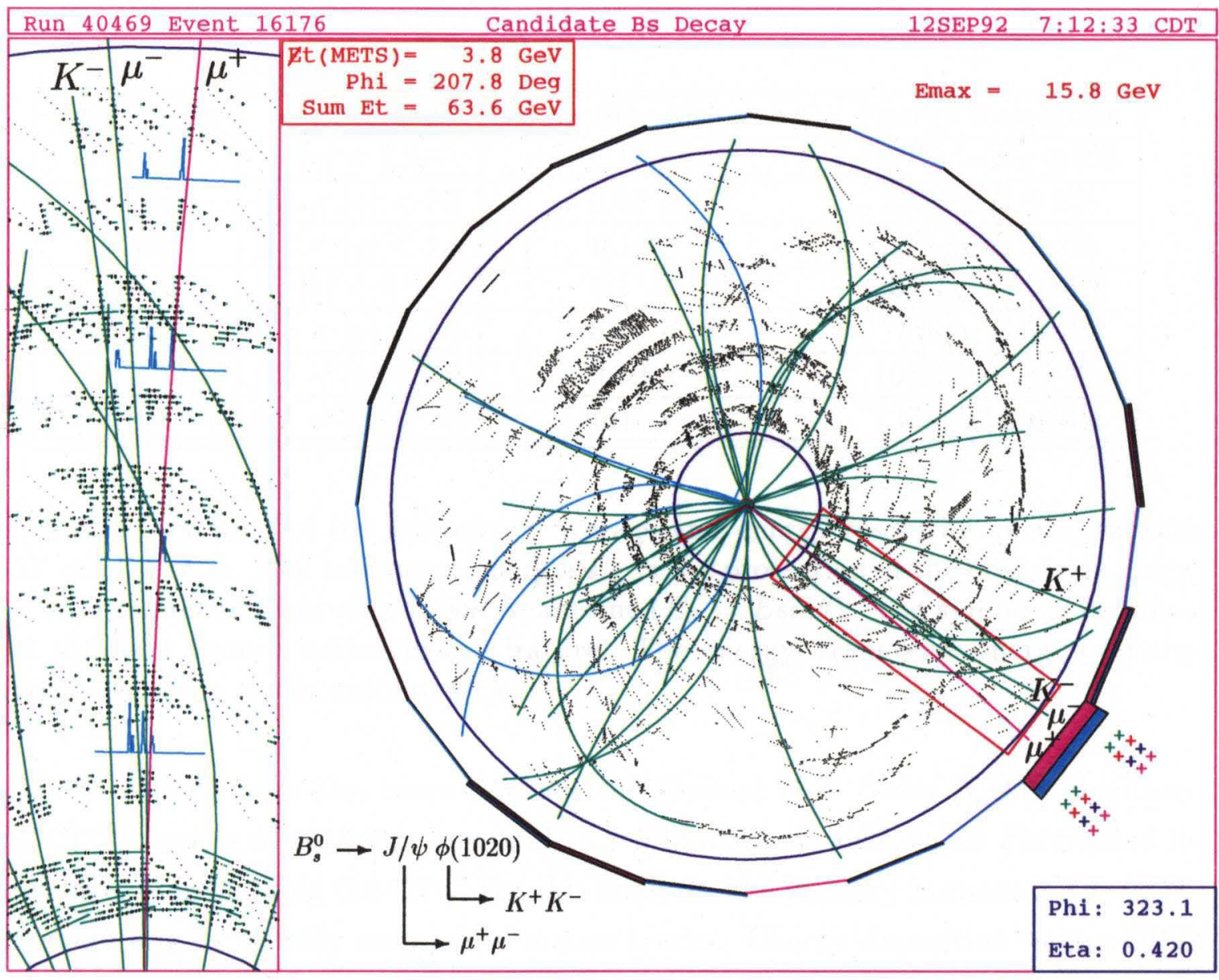

Figure 2.6: Event display of a candidate $B_{s}^{0}$ decay. The main display on the right shows the sense wire hits in black, and the transverse projection of the trajectory of the charged particles in the CTC chamber (magenta $=$ track with highest transverse momentum, green $=$ good fit, cyan $=$ unattached track). The inner blue circle represents the VTX boundary. The outer blue circle represents the solenoid. The ring of boxes around the solenoid represents the sum of transverse (magenta = electromagnetic, cyan $=$ hadronic) energy detected in each 15 degree wedge of the central calorimeters. The height of the boxes are scaled to the maximum energy shown in the upper right corner. The red arrow represents the transverse missing energy vector with the information shown in the upper left red box. The + marks represent hits in the central muon chambers with different colors for the different layers. The smaller display on the left is a close up of the red boxed region in the main display. The $\phi-\eta$ coordinate of the track with the highest transverse momentum is shown in the lower right blue box. The blue dots in the side window represent the position of the sense wires. The green dots represent the projected position of the charged track that produces a signal on the neighboring sense wires. The cyan spikes represent the CFT timing and position information. The header contains the run and event number as well as the date and time when the event was recorded. The distances between different detector components are not drawn to scale. 


\begin{tabular}{|c|c|c|c|}
\hline \hline Calorimeter & $\eta$ Coverage & Tower Size $(\Delta \eta \times \Delta \phi)$ & Energy Resolution \\
\hline CEM & $|\eta|<1.1$ & $0.1 \times 15^{\circ}$ & $13.7 \% / \sqrt{E_{T}} \oplus 2 \%$ \\
\hline PEM & $1.1<|\eta|<2.4$ & $0.09 \times 5^{\circ}$ & $22 \% / \sqrt{E} \oplus 2 \%$ \\
\hline FEM & $2.2<|\eta|<4.2$ & $0.1 \times 5^{\circ}$ & $26 \% / \sqrt{E} \oplus 2 \%$ \\
\hline CHA & $|\eta|<0.9$ & $0.1 \times 15^{\circ}$ & $50 \% / \sqrt{E_{T}} \oplus 3 \%$ \\
\hline WHA & $0.7<|\eta|<1.3$ & $0.1 \times 15^{\circ}$ & $75 \% / \sqrt{E} \oplus 4 \%$ \\
\hline PHA & $1.3<|\eta|<2.4$ & $0.09 \times 5^{\circ}$ & $106 \% / \sqrt{E} \oplus 6 \%$ \\
\hline FHA & $2.4<|\eta|<4.2$ & $0.1 \times 5^{\circ}$ & $137 \% / \sqrt{E} \oplus 3 \%$ \\
\hline
\end{tabular}

Table 2.1: Summary of the geometric coverage and energy resolution of the various CDF calorimeters. The transverse energy, $E_{T}$ is the projection of the observed energy $(E)$ onto the plane transverse to the beam line $\left(E_{T}=E \sin \theta\right)$. The symbol $\oplus$ signifies that the next term is added in quadrature. The energy, transverse energy and the energy resolution are in units of $\mathrm{GeV}$.

Further away from the beam line lies the Central Drift Tube (CDT) chamber. The CDT consists of $20133 \mathrm{~m}$ long and $12.7 \mathrm{~mm}$ diameter drift tubes distributed in three layers surrounding the CTC. For this analysis, the primary purpose of the CDT chambers was to identify cosmic ray muons tracks. Charge deposition in the CDT chambers at opposite ends in $\phi$ within a given time window signaled the presence of a cosmic ray muon. The muons were then used to determine the relative positions of the SVX, VTX and CTC chambers by comparing the fitted trajectories from the different chambers.

All of the above mentioned tracking chambers are contained within a superconducting solenoid magnetic coil. The solenoid magnetic coil is $5 \mathrm{~m}$ long and has a 3 $\mathrm{m}$ diameter and consists of 1164 turns of a $\mathrm{NbTi} / \mathrm{Cu}$ superconductor. The resulting field is nearly uniform and the strength is approximately 1.4 Tesla.

Around the solenoid and in front of the tracking chambers are numerous sampling calorimeters that are used to measure the electromagnetic energy of electrons and photons, and the hadronic energy of hadrons in jets (cluster of particles). The calorimeters surround the beam line and cover the pseudorapidity $\eta$ range from -4.2 to 4.2 (with cracks at $\eta=0,1.1$ and 2.4) in three regions, as can be seen in Figure 2.3. There are seven calorimeters, which are as follows: central electromagnetic (CEM), plug electromagnetic (PEM), forward electromagnetic (FEM), central hadronic (CHA), wall hadronic (WHA), plug hadronic (PHA) and forward hadronic 


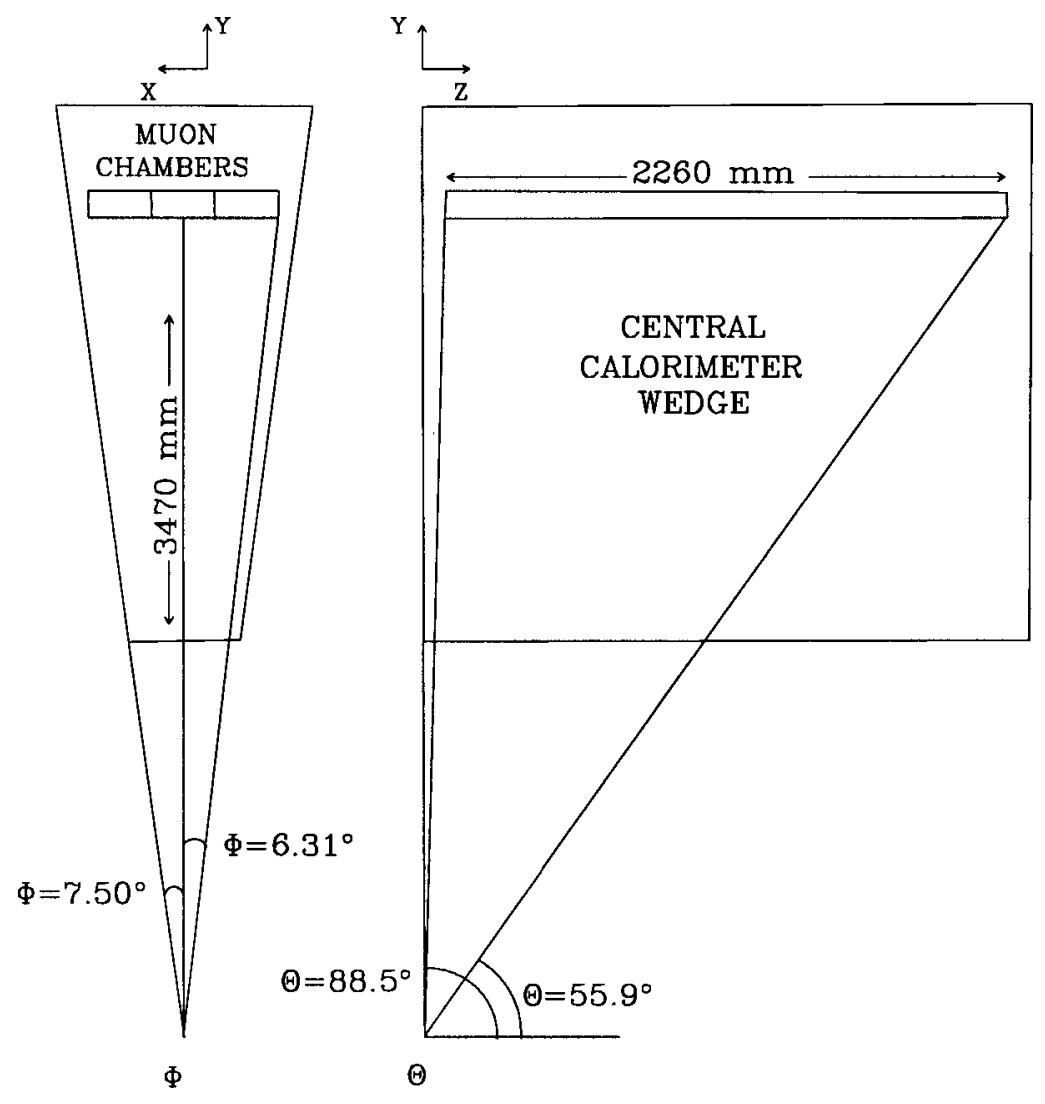

Figure 2.7: Layout of the central muon chambers within a central calorimeter wedge.

(FHA). All of the calorimeters are segmented in azimuth and pseudorapidity to form a projective tower geometry that has each tower pointing to the center of the detector. The pseudorapidity range, tower size and energy resolution for the seven calorimeters are summarized in Table 2.1. Located inside and in front of the CEM calorimeter are two shower position measurement chambers. The one in front of the CEM chamber and behind the solenoid is a proportional wire chamber (Central-Preradiator Chamber or CPR) that samples the $r$ - $\phi$ development of showers produced in the solenoid. The central electromagnetic strip (CES) detector is a proportional wire chamber with segmented cathode readout and is located inside the CEM chamber at the approximate position of maximum electromagnetic shower development. The CES detector provides $z$ and $r$ - $\phi$ electromagnetic shower position measurements.

The muon chambers are located behind the calorimeters. The material in the calorimeters filters out the various hadrons and electrons. There are three muon 
chambers in the central region. The chambers closest to the beam line are referred to as the Central Muon (CMU) drift chambers. The CMU drift chambers are attached to the outside of the CHA chambers at a radial distance of $347 \mathrm{~cm}$ and are segmented in $12.6^{\circ}$ wedges for each of the $15^{\circ}$ calorimeter wedges. Each chamber has three modules in $\phi$ as is shown in Figure 2.7. Each module is further segmented into four layers of four drift cells as can be seen in Figure 2.8. Two adjacent cells share the same sense wire (attached at $\eta=0$ ). The dimensions of each drift cell is $63.5 \mathrm{~mm}$ (wide) $\times 26.8$ $\mathrm{mm}$ (width) $\times 2261 \mathrm{~mm}$ (long) and covers the region given by $|\eta|<0.6$. For two of the four layers, the wires lie on a radial line that goes through the center of the CDF detector. The other wires lie on a radial line that is offset by $2 \mathrm{~mm}$. The alternate wires are offset so as to remove the ambiguity as to which side of the sense wires the track passes. Twice as many wires as needed were added to obtain a coarse but rapid angle determination of the muon candidates with the use of the time of arrival information of the pulses from the sense wires. This angle is then proportional to the total bending angle of the track, which is a function of the transverse momentum of the charged track. The $z$ position of the muon candidates can also be determined by measuring the difference of the charge deposited at the ends of the sense wire (at $\left.|\theta|=55.9^{\circ}\right)$.

A few years after the construction of the central muon chambers, two additional muon chambers were constructed. The first of these are the central muon upgrade (CMP) chambers, which consists of four layers of muon chambers located on top of and underneath the solenoid return yoke and on either side of the central detector. They form a box around the beam line, as shown in Figures 2.3 and 2.4. The CMP chambers are similar to the CMU chambers with the exception that each drift cell is longer than the CMU drift cell and the spacing between the muon chambers is not the same. The CMP drift cells are $320 \mathrm{~cm}$ along the beam line and the crack at $\theta= \pm 90^{\circ}$ is removed. The CMP chambers also cover some of the CMU $\phi$ cracks. In all, the CMU $\phi$ coverage is $84 \%$, the CMP $\phi$ coverage is $63 \%$ and $53 \%$ of the azimuth is covered by both.

The second additional muon chambers are the central muon extension (CMX) chambers. The CMX chambers have a conical arc shape, as shown in Figure 2.3. Each arc has four layers of drift cells with the same cross section as the CMU and CMP drift cells, but are considerably shorter. The addition of the CMX chambers increases the muon detection $\eta$ coverage from 0.6 to 1.0 , but the CMX chambers do 


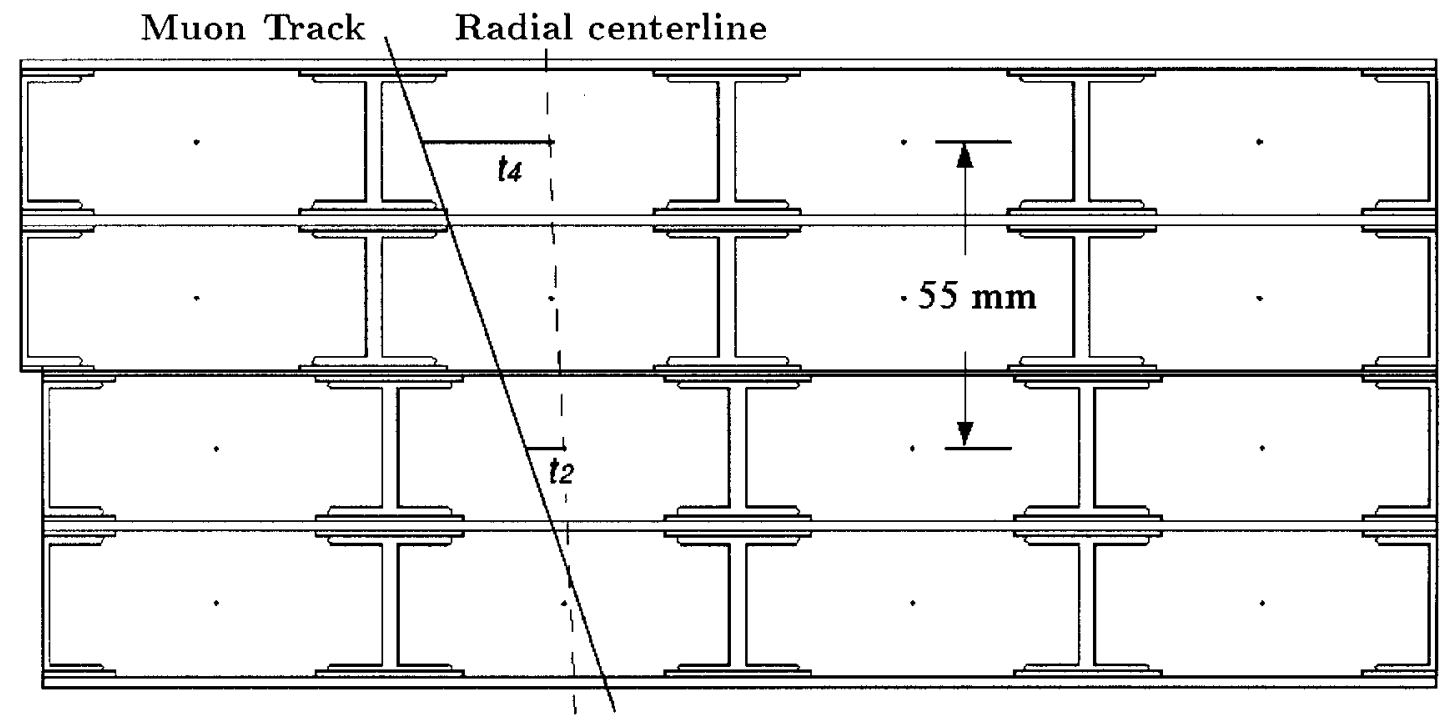

To $\bar{p} p$ interaction vertex

Figure 2.8: Layout of the central muon drift cells within a central muon chamber. The variables $t_{2}$ and $t_{4}$ are the time of arrival of the drift electrons for the second and fourth layers, which are aligned to the radial center line. Adjacent cells within the same layer share a sense wire. 
not provide full azimuthal coverage. The CMX arcs only span the $\phi$ interval between $-45 /+135$ degrees to $+75 /+255$ degrees or $71 \%$ of $2 \pi$.

At the far end of the forward-backward region lie the forward muon (FMU) detection system. The FMU system consists of a pair of magnetized iron toroids instrumented with three sets of drift chambers and two planes of scintillation counters. The rapidity coverage of the FMU is $2.0<|\eta|<3.6$. Because the $\eta$ coverage of the FMU is outside the range of the CTC and the intrinsic momentum resolution of the FMU system is poor, the FMU was not used in this analysis.

The forward-backward region also contains the beam-beam counters $(\mathrm{BBC})$, which consist of two planes of scintillation counters covering the angular range $3.24<|\eta|<$ 5.88. The BBC have two primary functions. They provide a minimum-bias trigger that accepts events if there are hits in both the forward and backward BBC in coincidence with the $\bar{p} p$ bunch crossings. The BBC also serves as a luminosity monitor. The instantaneous luminosity is obtained by dividing the minimum bias trigger rate by the measured BBC cross section. More complete descriptions of the BBC counters and the other components of the CDF detector can be found in References $[48,49,50,51]$ and the references within.

\subsection{The CDF Trigger System}

With proton and antiproton bunches passing each other every $3.5 \mu$ s at the B0 interaction point at a typical instantaneous luminosity of $5 \times 10^{30} \mathrm{~cm}^{-2} \mathrm{~s}^{-1}$, the rate of collisions is very high. There are approximately 0.8 collisions per beam crossing given the total $\bar{p} p$ cross section at $\sqrt{s}=1.8 \mathrm{TeV}$ of $(80.03 \pm 2.24) \times 10^{-27} \mathrm{~cm}^{2}[52]$. At that rate, it is difficult and costly to store the 165 thousand bytes of information per collision that the CDF detector produces on storage media that can be later used for data analysis. As a result, a multi-level trigger is used to select only the most interesting collisions, such as $b$ quark decays with transverse momentum greater than $8 \mathrm{GeV} / c$ and $|\eta|<1.0$, which on the average are produced with a frequency of $40 \mathrm{~Hz}$.

The general requirements of this trigger system are i) to reduce the event acceptance rate from the initial rate of $286 \mathrm{kHz}$ down to a rate under $8 \mathrm{~Hz}$, the maximum feasible data writing speed to a storage medium and ii) to accept as many interesting events as possible without incurring large amounts of dead time (the time in which the detector is unable to consider more events). Reading out all the data consumes 
over $1 \mathrm{~ms}$, which would cause at least $99.65 \%$ of the beam crossings to not be analyzed if there was no trigger. To increase the number of beam crossings that are analyzed, a three level trigger system is used, with each successive level making more detailed requirements based on more information and using more processing time. The decision at each of the three levels is a logical OR of many requirements that are designed to select different physics processes. Some of the requirements are programmable so that they can be changed for different running conditions. For the first two levels, the trigger information is obtained from the various sub-detectors through dedicated signal lines.

\subsubsection{Level 1}

The level 1 trigger is designed to be very fast. The decision on whether the event should be considered for the next level is made within the $3.5 \mu$ s beam crossing time. The level 1 requirements can be broken down into three groups.

The first is the requirement that the forward and backward BBC counters have hits in coincidence with the $\bar{p} p$ crossings. For the first half of the data run this requirement was always used in conjunction with the other two types of physics requirements. This requirement was not used in the second half of the data run because the instantaneous luminosity was high enough to cause this criteria to be satisfied virtually all of the time.

The second group of requirements are the decisions made on calorimetry measurements. These calorimetry measurements are based on the energy sum from "trigger towers", set of calorimetry towers encompassing the area given by $(\Delta \eta \times \Delta \phi=$ $\left.0.2 \times 15^{0}\right)$. If the sum of electromagnetic or hadronic energy in the trigger towers is above a certain programmable energy value (which can differ for the different calorimeter modules) the event passes the level 1 trigger.

The third group of requirements is based on the identification of hits in the muon

chambers. For this analysis only hits in the CMU, CMP and CMX chambers are considered. Since all of the decays used in this analysis involved two muons from the $J / \psi \rightarrow \mu^{+} \mu^{-}$decay, a coincidence of charge deposition on sense wires from two different muon "towers" are required. A muon tower is defined to be four layers of two columns of muon drift tubes. The layout of the muon chambers shown in Figure 2.8 contains two muon trigger towers. Two adjacent muon drift cells in each layer share the same sense wire. For each muon trigger tower, two sense wires lie along the 
radial center line and two sense wires are displaced from the radial center wire by $2 \mathrm{~mm}$. The two types of wire alternate between layers. The muon trigger requires that there be charge deposition on at least both radial wires or both shifted wires. In addition, the difference between the time of arrival of the pulses from either type of sense wires are required to be less than a preset value for each muon candidate. This requirement is an effective minimum transverse momentum cut of $1 \mathrm{GeV} / c$. Because charged tracks bend in the presence of an axial magnetic field, the muon tracks will traverse the muon chambers at an angle with respect to the radial line. This angle, which is shown in Figure 2.8, can be calculated by taking the difference in the time of arrival of the pulses from the sense wires $\left(\left|t_{4}-t_{2}\right|\right.$ or $\left.\left|t_{3}-t_{1}\right|\right)$. The angle is itself inversely proportional to the transverse momentum of the particle. For the CMX chambers, a coincidence of hits in slabs of plastic scintillators before and after the chambers is also required. For hits in the CMP chambers that overlap the fiducial area of the CMU cells, a coincidence of hits in both the CMU and CMP cells is also required.

Overall, the combined level 1 acceptance rate is approximately $1 \mathrm{kHz}$ at a typical luminosity of $5 \times 10^{30} \mathrm{~cm}^{-2} \mathrm{~s}^{-1}$. When any of the level 1 trigger requirements are satisfied, a subset of the data is checked to see if they satisfy any of the second level trigger requirements. During this time, no other interaction is considered.

\subsubsection{Level 2}

The level 2 trigger is designed to identify physics "objects" such as electrons, photons, taus, neutrinos using missing transverse energy, muons and jets. The decision on whether the event should be further considered is made within approximately $20 \mu \mathrm{sec}$, which is the dead time associated with the level two trigger. The level 2 requirements are based on information that is calculated at the level 1 stage as well as additional information described below.

At the level 2 stage, a CTC fast track (CFT) pattern recognition algorithm is used to measure the approximate $\phi$ and transverse momentum of the charge tracks that traverse the CTC chamber. The CFT is a hardware track processor that uses fast timing information from the axial CTC super-layers to determine the drift time of the electrons that are stripped from the gas within the CTC chamber. Because the drift time is proportional to the location at which the ionization occurred, one can determine the trajectory of the particles given the timing information. Two sets of 
timing information relative to the beam crossing times are obtained from the wires in each axial super-layer. Given the timing information, the transverse momentum is then digitized into eight $P_{T}$ bins with nominal central values of $3.3,4.0,5.0,6.5$, $10.0,15.0,20.0$, and $30.0 \mathrm{GeV} / c$. The transverse momentum resolution at this stage is $\delta P_{T} / P_{T}^{2} \sim 3.5 \%$, where $P_{T}$ has units of $\mathrm{GeV} / c$.

Calorimeter energy clustering is also performed at the level 2 stage. An algorithm checks for calorimeter trigger towers that have some energy deposition above two sets of energy thresholds ("seed" and "shoulder"). A calorimeter cluster is formed when any trigger tower passes the seed threshold. Any of the four neighboring trigger towers in the $(\eta, \phi)$ plane are added to the cluster if their respective tower energy is greater than the shoulder threshold. Additional neighboring towers can be further added to the cluster, if the towers surpass the shoulder threshold and the towers do not belong to a different cluster. Once the clustering is performed, the $E_{T}$, average $\phi$ and $\eta$ are calculated for each cluster. Global sums of calorimetric energy such as total energy, total transverse energy, total missing transverse energy and total clustered energy are also calculated. Electromagnetic and hadronic energy clustering can be used separately for triggering of jets or for photon identification. Electron candidates can be identified with the requirement that a CFT track points to an electromagnetic cluster. Neutrino candidates can be identified with the use of missing transverse energy information.

To further reduce the rate of triggers due to the level 1 central muon trigger accepts ( $\sim 400 \mathrm{~Hz}$ at level 1$)$, the central muon hits are required to be matched to CFT tracks at the level 2 stage. The matching requirements are made in the transverse plane. The muon hits and the extrapolated CFT track were required to be within $15^{\circ}$ in $\phi$. At the level 2 stage, $J / \psi \rightarrow \mu^{+} \mu^{-}$candidate events are triggered with the requirement that at least one of the muons is matched with a CFT track. The allowed combinations are:

- Two CMU muons, with either CMU track matching a CFT track.

- One CMU muon and one CMX muon, with the CMU track matching a CFT track.

- One CMU muon and one CMX muon, with the CMX track matching a CFT track. 
- Two CMX muons, with either CMX track matching a CFT track.

The last two requirements were only added during the second half of the data run.

Overall, the combined level 2 acceptance rate is approximately $12 \mathrm{~Hz}$ at a typical luminosity of $5 \times 10^{30} \mathrm{~cm}^{-2} \mathrm{~s}^{-1}$. When any of the level 2 trigger requirements are satisfied all of the detector channels are digitized and the information is transferred to the level 3 trigger. The digitization takes over $3 \mathrm{~ms}$. Once the digitization is complete, the detector is free to examine more $\bar{p} p$ collisions starting with another level 1 trigger.

\subsubsection{Level 3}

The level 3 trigger system is designed to handle multiple events at the same time and to reduce the trigger rate to below $8 \mathrm{~Hz}$. The system consists of 48 commercial processors (from Silicon Graphics Inc. running with the IRIX operating system). The combined processing power is approximately 1.1 billion instructions per second. The system also contains 96 memory buffers with a combined capacity of approximately 0.5 Gigabytes. There are twice as many memory buffers as there are processors, so as to be able to process simultaneously 48 events while writing (reading) 48 independent events into (from) memory.

At the level 3 stage, full CTC track reconstruction is performed (all of the five track parameters are calculated). At this stage, two additional requirements are applied to the $J / \psi \rightarrow \mu \mu$ candidate events. The first is the requirement that the hits in the muon chambers and an extrapolated CTC track match in the $r$ - $\phi$ and $z$ plane within four standard deviations, taking into account uncertainties due to multiple scattering and measurement resolutions. Both muon candidates are required to satisfy this condition. The second requirement is that the invariant mass of the two muons lie between $2.8 \mathrm{GeV} / c^{2}$ and $3.4 \mathrm{GeV} / c^{2}$.

Overall, the combined level 3 acceptance rate is approximately $5 \mathrm{~Hz}$ at a typical luminosity of $5 \times 10^{30} \mathrm{~cm}^{-2} \mathrm{~s}^{-1}$. When any of the level 3 trigger requirements are satisfied all of the detector information and the trigger information are written to one of four $8 \mathrm{~mm}$ tape drives. The typical raw data event size consists of approximately 165 kilobytes of information. For a subsample of low frequency triggers, such as the $J / \psi \rightarrow \mu^{+} \mu^{-}$trigger, the events were also written directly to a disk drive for fast access and analysis. 


\section{Chapter 3}

\section{Event Selection}

The data used for this analysis were collected during an eight month period with the Tevatron collider operating at $\sqrt{s}=1.8 \mathrm{TeV}$. The run started on August 26, 1992 and ended on May 30, 1993. The integrated luminosity of this run corresponds to $19.6 \mathrm{pb}^{-1}$.

Of all of the events that were collected and stored on $8 \mathrm{~mm}$ tape, only those events that passed the dimuon third level trigger were analyzed for this study.

Because of the large amounts of backgrounds that are inherent in hadron-hadron collisions, further requirements were applied to reduce the backgrounds while retaining a large percentage of the desired events. These requirements or "cuts" are discussed in the following sections.

The code that was used to select all of the decays used in this analysis was written in FORTRAN and was run on a similar computer architecture to the one that was used for the third level of the trigger. The algorithm encoded to select all the events used in this analysis can reconstruct decays of the general form

$$
D_{i+2} \rightarrow D_{i+1}+D_{i}
$$

where $D_{i}$ can be any charged particle or any neutral particle that eventually decays to charged daughters. The benefit of using only one algorithm is that one is assured that each decay is treated equally. A second important feature of the algorithm is its recursive structure. The output of one sequence could be fed into another and thus one can easily reconstruct complicated decay such as the $B^{+} \rightarrow J / \psi K^{*}(892)^{+}$decay that is shown in Figure 3.1. 


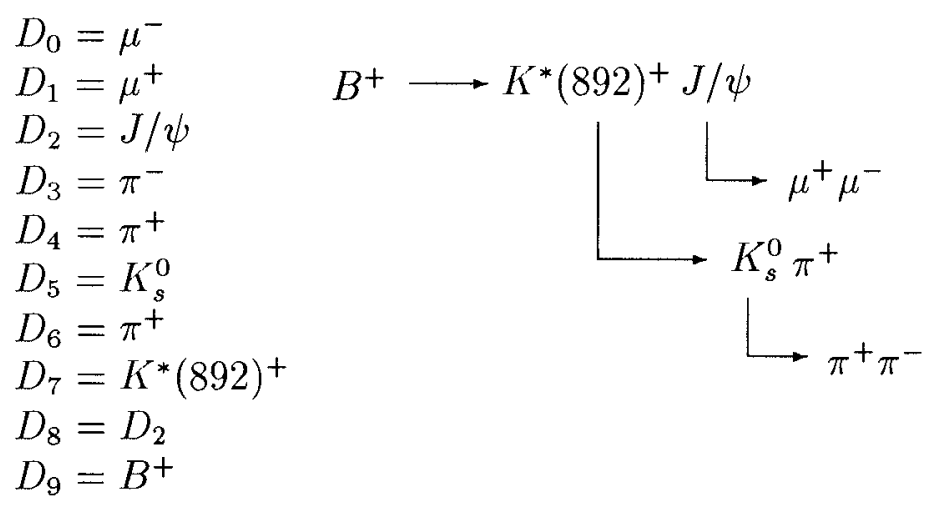

Figure 3.1: Decay chain of the reconstructed $B^{+}$meson into a $J / \psi K^{*}(892)^{+}$pair.

\subsection{Track Quality Requirements}

As mentioned earlier, additional requirements were applied to reduce backgrounds. Some of the backgrounds present are due to poorly measured tracks. In order to ensure that the track parameters were well measured, a specific set of track quality requirements were made.

All of the charged tracks were required to have a transverse momentum $\left(P_{T}\right)$ larger than $400 \mathrm{MeV} / c$. This cut was applied to eliminate tracks that did not traverse the entire CTC chamber. Tracks with momentum less than $280 \mathrm{MeV} / c$ will curl inside the CTC and will not reach the outer CTC can. Such tracks will not be well measured since the number of hits available in the outer layers will be reduced. It should be noted that this requirement was made before any multiple scattering or $d E / d x$ corrections were applied. Multiple scattering and $d E / d x$ corrections were applied at a later stage and perturb the track momentum by a few $\mathrm{MeV} / \mathrm{c}$.

Tracks were required to have at least 4 hits in at least $2 \mathrm{CTC}$ axial super-layers and at least 2 hits in at least 2 CTC stereo super-layers. In addition, tracks that exited through the CTC endplates were not used for this analysis. These requirements were applied to eliminate poorly measured tracks.

With the addition of the SVX tracking chamber, there are two sets of tracking information that one can use. In the first set, the tracking parameters are obtained solely with the use of the CTC chamber. The second set of tracking parameters are obtained from a combined fit of information from both the SVX and CTC chambers. 
Ideally, the combined SVX-CTC information is best, but there are occasions when the SVX track information is poor enough that it is better to only use the CTC information. Various selection criteria were analyzed to determine at what point it becomes beneficial to used the combined SVX-CTC fit information. The various criteria that were studied are listed below:

1. Use only CTC fit information.

2. Use SVX-CTC fit information if SVX information is available.

3. Use SVX-CTC fit information if $\chi^{2}$ (SVX fit) $<20.0$.

4. Use SVX-CTC fit information if $\chi^{2} / \mathrm{Ndof}(\mathrm{SVX}$ fit $)<6.0$.

5. Use SVX-CTC fit if C.L.(SVX fit) $>0.001$.

The quantity $\chi^{2}$ (SVX fit) is defined to be the increase in the track fit $\chi^{2}$ when the SVX hits are included in the CTC track fit. Only one SVX track is allowed to be associated with a CTC track. In addition, at least three SVX hits for each SVX fit was required.

To determine the best selection requirement, three different quantities were examined from two different decays. The quantities include the number of fitted events, the standard deviations of two Gaussian line shapes representing the signal, and the ratio of signal events to background events for $J / \psi \rightarrow \mu^{+} \mu^{-}$and $K_{s}^{0} \rightarrow \pi^{+} \pi^{-}$decays. The selection requirement that would improve the three quantities for both decays would then be expected to be useful in other decays.

For the $J / \psi$ invariant mass spectra, a double Gaussian with a mass dependent amplitude term was fitted to the signal region. The second Gaussian was needed to obtain a reasonable fit of the mass spectra. The mass dependent amplitude term was added to account for the asymmetry of the mass distribution due to QED radiative effects. The double Gaussian had a common mean and the background was approximated by a linear term. The $K_{s}^{0}$ invariant mass distribution was fitted in the same way except there was no mass dependent amplitude term. The fitted parameters for the two distributions are shown in Tables 3.1 and 3.2 .

The fitted parameters were obtained with the use of the MN_FIT program (version $3.02 / 02$ of the executable was used). The MNFIT program does various fits of histograms with the use of MINUIT [53] minimization algorithm. All the histogram fits 


\begin{tabular}{|c|c|c|c|c|}
\hline \hline Method \# & \# of Events & $\sigma_{1}\left(\mathrm{MeV} / c^{2}\right)$ & $\sigma_{2}\left(\mathrm{MeV} / c^{2}\right)$ & Signal/Background \\
\hline \hline 1 & $78261 \pm 282$ & $17.8 \pm 0.2$ & $37.9 \pm 0.7$ & 4.37 \\
\hline 2 & $77952 \pm 311$ & $14.6 \pm 0.1$ & $33.3 \pm 0.6$ & 4.37 \\
\hline 3 & $78094 \pm 362$ & $14.6 \pm 0.1$ & $33.3 \pm 0.6$ & 4.38 \\
\hline 4 & $78042 \pm 253$ & $14.6 \pm 0.1$ & $33.2 \pm 0.6$ & 4.38 \\
\hline 5 & $78034 \pm 345$ & $14.6 \pm 0.1$ & $33.3 \pm 0.6$ & 4.37 \\
\hline \hline
\end{tabular}

Table 3.1: Variations of some fitted parameters on the inclusive $J / \psi$ invariant mass spectrum for different SVX requirements. The parameters $\sigma_{1}$ and $\sigma_{2}$ are the smaller and larger standard deviations of the double Gaussian line shape of the signal region.

\begin{tabular}{|c|c|c|c|c|}
\hline \hline Method \# & \# of Events & $\sigma_{1}\left(\mathrm{MeV} / c^{2}\right)$ & $\sigma_{2}\left(\mathrm{MeV} / c^{2}\right)$ & Signal/Background \\
\hline \hline 1 & $45515 \pm 895$ & $3.5 \pm 0.2$ & $10.6 \pm 0.6$ & 0.097 \\
\hline 2 & $43432 \pm 856$ & $3.3 \pm 0.2$ & $9.8 \pm 0.6$ & 0.093 \\
\hline 3 & $44147 \pm 888$ & $3.3 \pm 0.2$ & $10.1 \pm 0.6$ & 0.094 \\
\hline 4 & $44051 \pm 845$ & $3.3 \pm 0.2$ & $9.9 \pm 0.6$ & 0.094 \\
\hline 5 & $44062 \pm 874$ & $3.3 \pm 0.2$ & $10.0 \pm 0.6$ & 0.094 \\
\hline \hline
\end{tabular}

Table 3.2: Variations of some fitted parameters on the inclusive $K_{s}^{0}$ invariant mass spectrum for different SVX requirements. The parameters $\sigma_{1}$ and $\sigma_{2}$ are the smaller and larger standard deviations of the double Gaussian line shape of the signal region.

in this analysis were done with the MN_FIT executable. The default fitting method used $\chi^{2}$ minimization for histograms with more than 1000 events and a maximum log-likelihood for histograms containing fewer than 1000 events.

From Table 3.1 one can see that only method 1 is not optimal because of its large standard deviation. There is very little difference between methods 2 through 5 . From Table 3.2 one only notices small changes between the various methods. In short, Table 3.2 shows that long lived particles such as the $K_{s}^{0}$ are mostly dependent on CTC information, so their reconstruction is insensitive to the type of SVX requirements used.

Since method 4 was used in the CDF top quark analysis [54], and from Table 3.1 it seems to be a reasonable requirement, it is used on all the other decays. In addition, the efficiency of this requirement is approximately equal for the two completely different topologies that have been studied. 

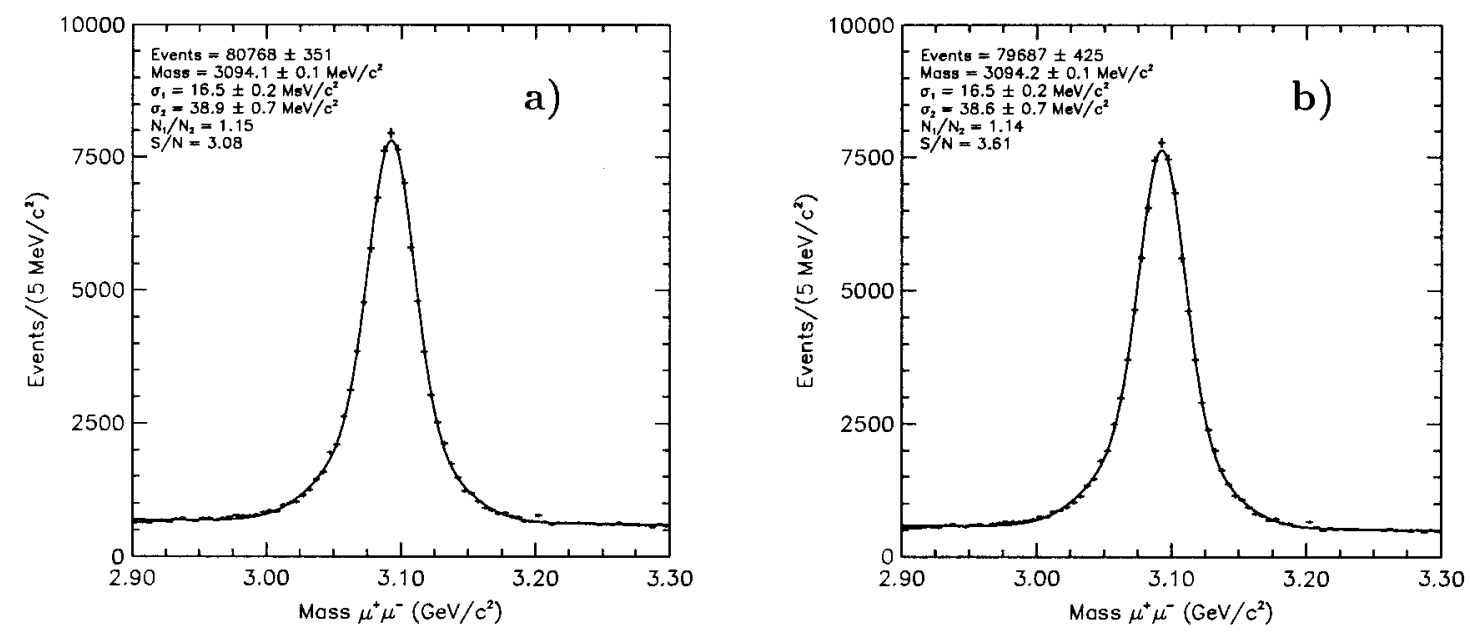

Figure 3.2: Invariant mass distribution of $\mu^{+} \mu^{-}$pairs (a) before and (b) after the muon segment CTC matching requirements are applied. Multiple scattering and $d E / d x$ corrections are not applied. The spectra were fitted to a double Gaussian with a mass dependent amplitude and a linear background term.

\subsection{Muon and $J / \psi$ Requirements}

A number of cuts were applied to the muon and $J / \psi$ candidates to reduce the backgrounds from non- $J / \psi$ decays. First, the two muons were required to have the opposite charge. Each muon was also required to have a minimum momentum of $1.4 \mathrm{GeV} / c$. This requirement was made because only muons with at least that momentum are likely to pass through the calorimetry at $z=0$ and $\eta=0$.

Each muon track was also required to match one and only one CTC track. The CTC track, when extrapolated to the muon chambers, was required to match within three standard deviations the muon stub in both the transverse plane $(r-\phi)$ and along the beam axis $(z)$. The extrapolation uncertainties included measurement and multiple scattering effects. For CTC tracks that had stubs in multiple muon chambers, a logical OR of the respective requirements was done. This cut is highly efficient for real muons and rejects a noticeable number of background events. Figure 3.2 shows the effect of the cut on the dimuon invariant mass distribution. The signal to noise ratio between $3.0 \mathrm{GeV} / c^{2}$ and $3.2 \mathrm{GeV} / c^{2}$ goes from 3.08 to 3.61 , while the efficiency for this requirement is $(98.66 \pm 0.04) \%$.

In order to improve the mass resolution of the $J / \psi$ resonance and decrease the 
number of background events in the signal region, the two muons were constrained to originate from the same vertex. The idea behind the constraint is to reduce the measurement errors by applying known physical constraints. This was done by performing a least-squares fit of the two muon candidate tracks, constraining the two tracks to come from a common point (a vertex constraint). Multiple scattering and $d E / d x$ corrections were also included in the fitting procedure. The probability of the fit was required to be greater than $1 \%$ (confidence level (C.L.) greater than 0.01). The power of the vertex constraint can be seen by comparing Figures 3.2 (b) and 3.3 (a). One can see that the vertex requirement is highly efficient while at the same time it improves the mass resolution of the resonance and the signal to noise ratio.

The $J / \psi$ invariant mass resonance plots were fitted to a double Gaussian with a linear mass dependent amplitude term and a common mean. The background was assumed to be linear, or the same as the like-charged muon invariant mass distribution. For the normalized mass distributions (difference between the measured mass and the world average mass divided by the uncertainty on the measured mass), the background was also assumed to be Gaussian or the same as the like-charged muon normalized mass distribution. All the world average masses and lifetimes were taken from the Particle Data Group (PDG) [7].

The mass dependent amplitude was used to account for the noticeable asymmetry of the $J / \psi$ invariant mass distribution. The asymmetry results from internal and external QED radiation. When the effect of radiation is included the reconstructed mean $J / \psi$ mass is between 3095.7 and $3095.9 \mathrm{MeV} / c^{2}$, which agrees with the results from the fits shown in Figures 3.3 and 3.5. Since this analysis is not dependent on mass values, no corrections to account for the $1000 \mathrm{KeV} / \mathrm{c}^{2}$ underestimate of the reconstructed $J / \psi$ mass is applied (the world average $J / \psi$ mass is $3096.88 \mathrm{MeV} / c^{2}$ ).

One can measure the efficiency of the C.L. $\left(\chi^{2}\right)>0.01$ requirement by taking the ratio of fitted events from either invariant mass or normalized mass distributions with and without the requirements. In addition, the efficiency can be determined with the use of the fitted confidence level distribution as shown in Figure 3.7. The efficiency is determined by taking the ratio of the integrated line in the cut region $\left(C . L .\left(\chi^{2}\right)>0.01\right)$ over the entire region.

The distributions that were used to determine the efficiency of the C.L. $\left(\chi^{2}\right)>0.01$ requirement using the various methods described above are given in Figures 3.3 to 3.7. The mean efficiency using the distributions in the five figures is $(97.8 \pm 0.8) \%$ 

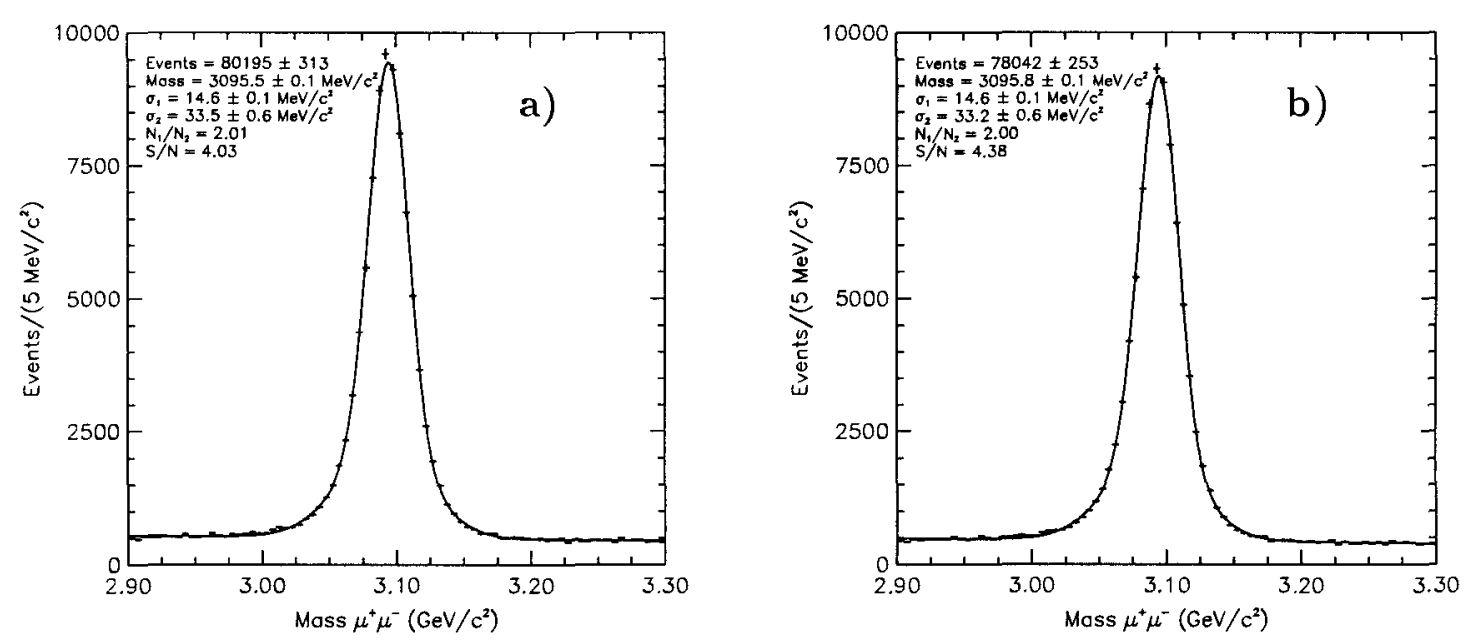

Figure 3.3: Invariant mass distribution of $\mu^{+} \mu^{-}$pairs (a) before and (b) after the confidence level requirement is applied when the dimuons are vertex constrained. The spectra were fitted to a double Gaussian with a mass dependent amplitude with linear background term.
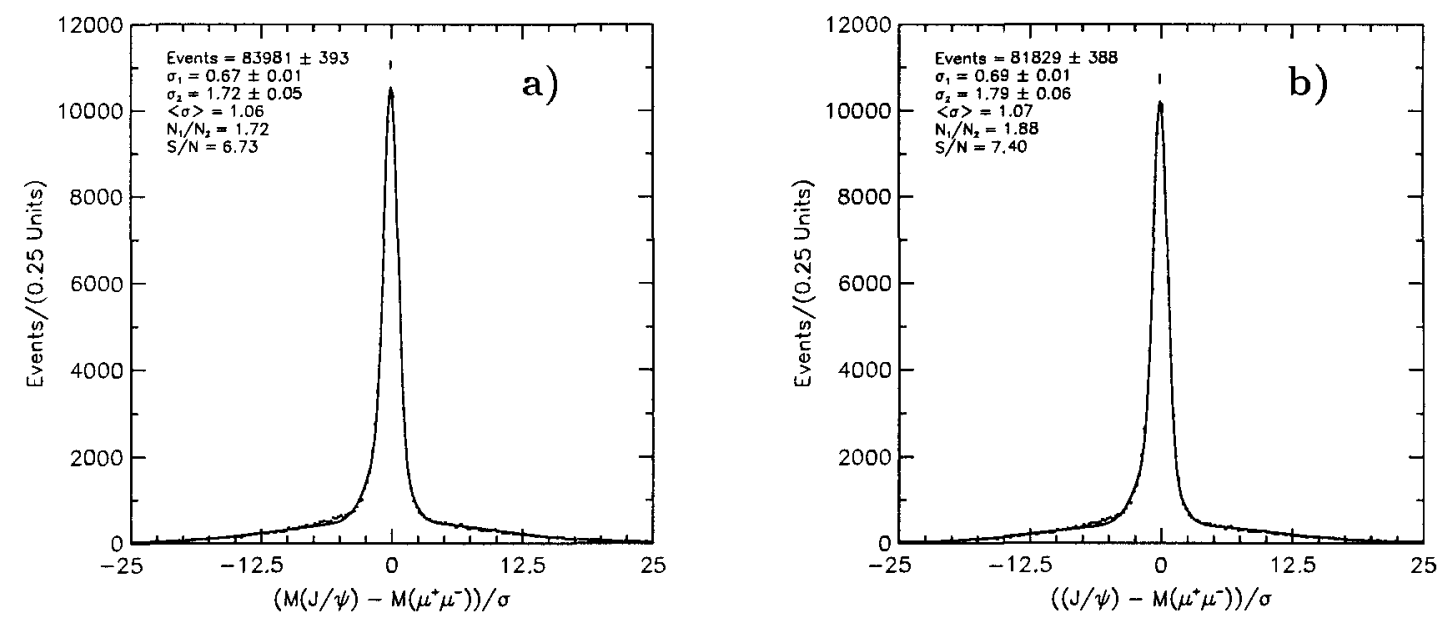

Figure 3.4: Normalized invariant mass distribution of $\mu^{+} \mu^{-}$pairs (a) before and (b) after the confidence level requirement is applied when the dimuons are vertex constrained. The spectra were fitted to a double Gaussian with a mass dependent amplitude and a Gaussian background term. 

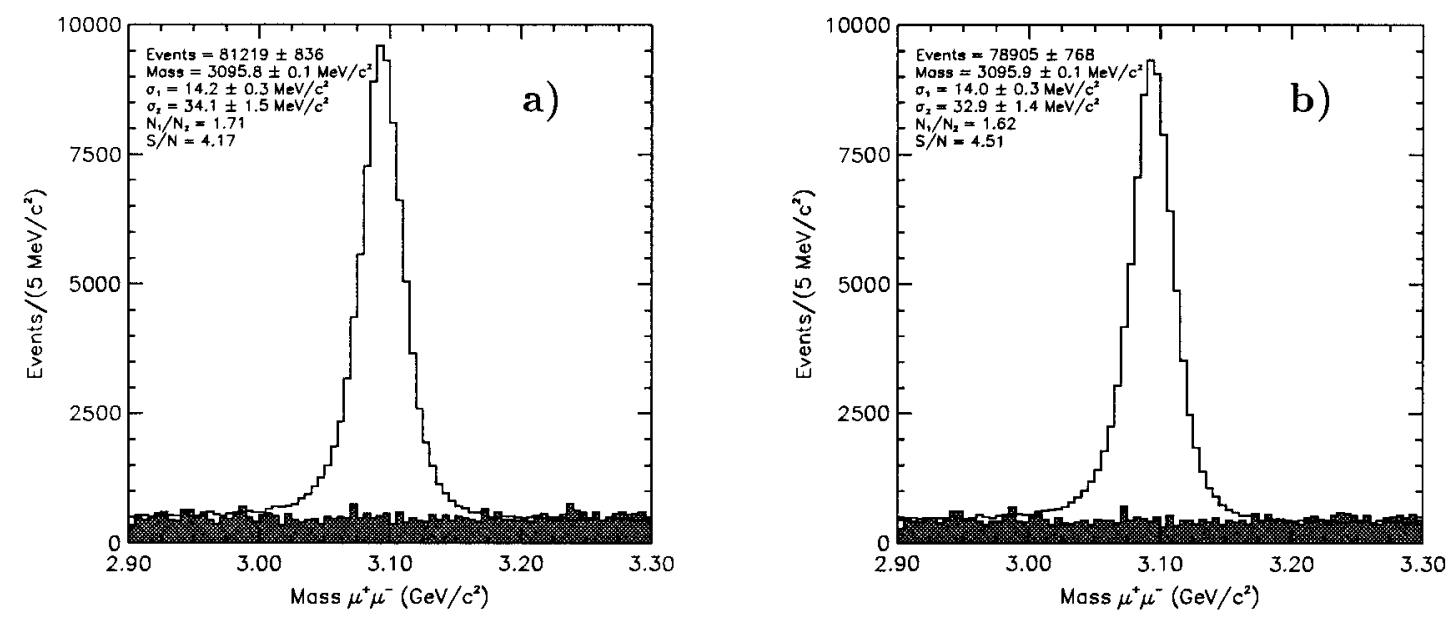

Figure 3.5: Invariant mass distribution of $\mu^{+} \mu^{-}$pairs (a) before and (b) after the confidence level requirement is applied when the dimuons are vertex constrained. The shaded region represents the invariant mass distribution for the same sign muon pairs. The spectra were fitted to a double Gaussian with a mass dependent amplitude and the background was normalized to the $\mu^{ \pm} \mu^{ \pm}$invariant mass distribution.
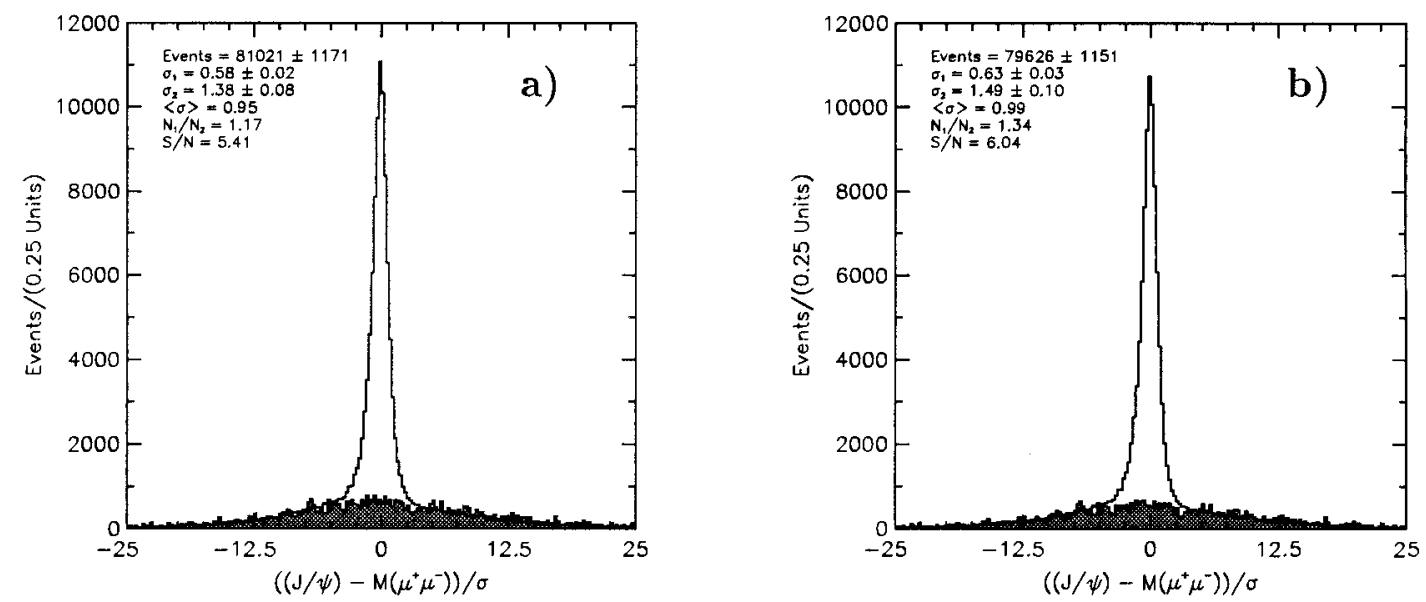

Figure 3.6: Normalized invariant mass distribution of $\mu^{+} \mu^{-}$pairs (a) before and (b) after the confidence level requirement is applied when the dimuons are vertex constrained. The shaded region represents the normalized invariant mass distribution for the same sign muon pairs. The spectra were fitted to a double Gaussian with a mass dependent amplitude and the background was normalized to the $\mu^{ \pm} \mu^{ \pm}$normalized invariant mass distribution. 

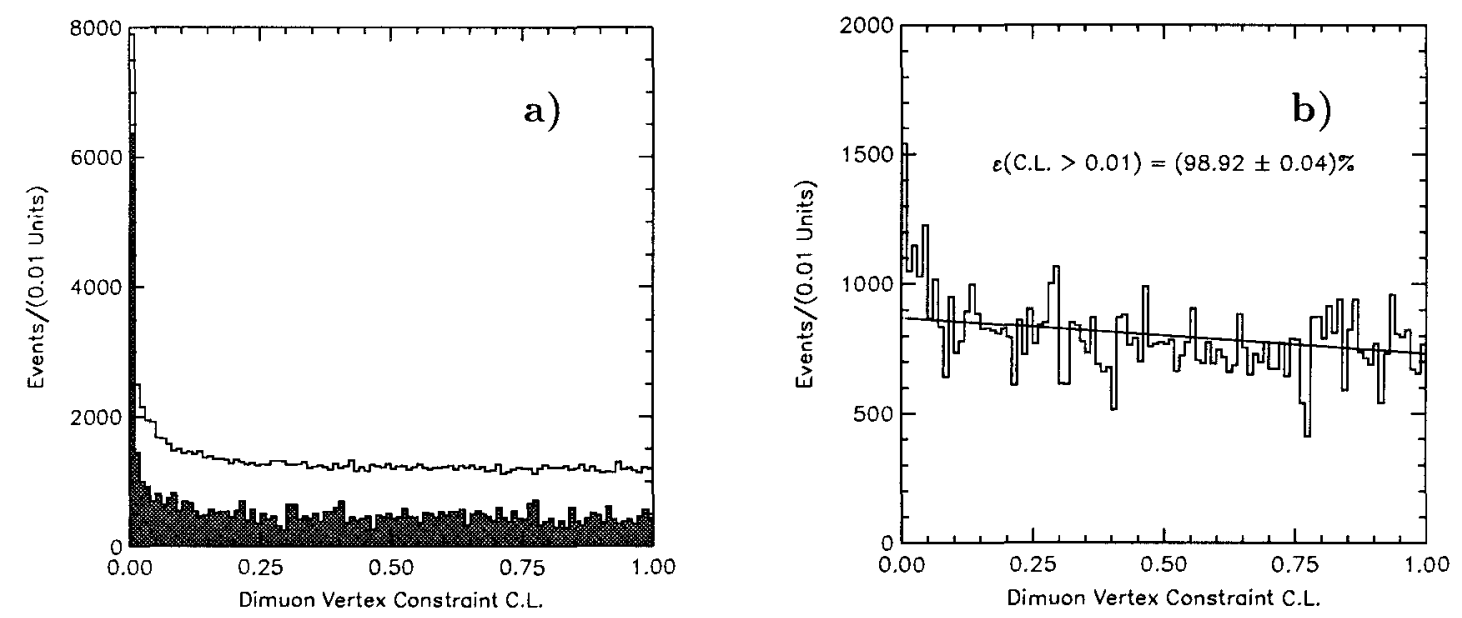

Figure 3.7: The confidence level distribution of the dimuon vertex fit. The shaded region represents the confidence level of the fit for the same charge dimuons. Figures (a) and (b) represent the distributions before and after a background subtraction is made. The efficiency is obtained by taking the ratio of the integrated line between 0.01 and 1.0 and the entire range.

where the error is the standard deviation for the five different values of the efficiency. The mean efficiency is approximately equal to the expected value of $99 \%$. There is however, a $1 \%$ inconsistency in the efficiency of the cut, but it is negligible for this analysis since only the ratio of efficiencies matters. This small inconsistency can be seen in Figure 3.7 (b), where the first bin is about 700 events higher $(0.9 \%$ of the total) than what it is expected to be. The approximate flatness of the confidence level distribution is a good indication that the covariance matrix of the fitted track parameters adequately represents the uncertainties of the track fit.

To further improve the $\mu^{+} \mu^{-}$invariant mass resolution and decrease the number of background events in the signal region, another constrainted fit was performed applying the vertex constraint and the constraint that the dimuon invariant mass be equal to the world average $J / \psi$ mass [7]. As before, the confidence level of the fit was required to be greater than $1 \%$.

The efficiency of the vertex and mass C.L. $\left(\chi^{2}\right)>0.01$ cut is harder to measure because of the asymmetric dimuon mass distribution. Nevertheless, several methods can be used to extract this efficiency and the associated uncertainty. The simplest method involves extrapolating a fitted line from the high end of the confidence level 

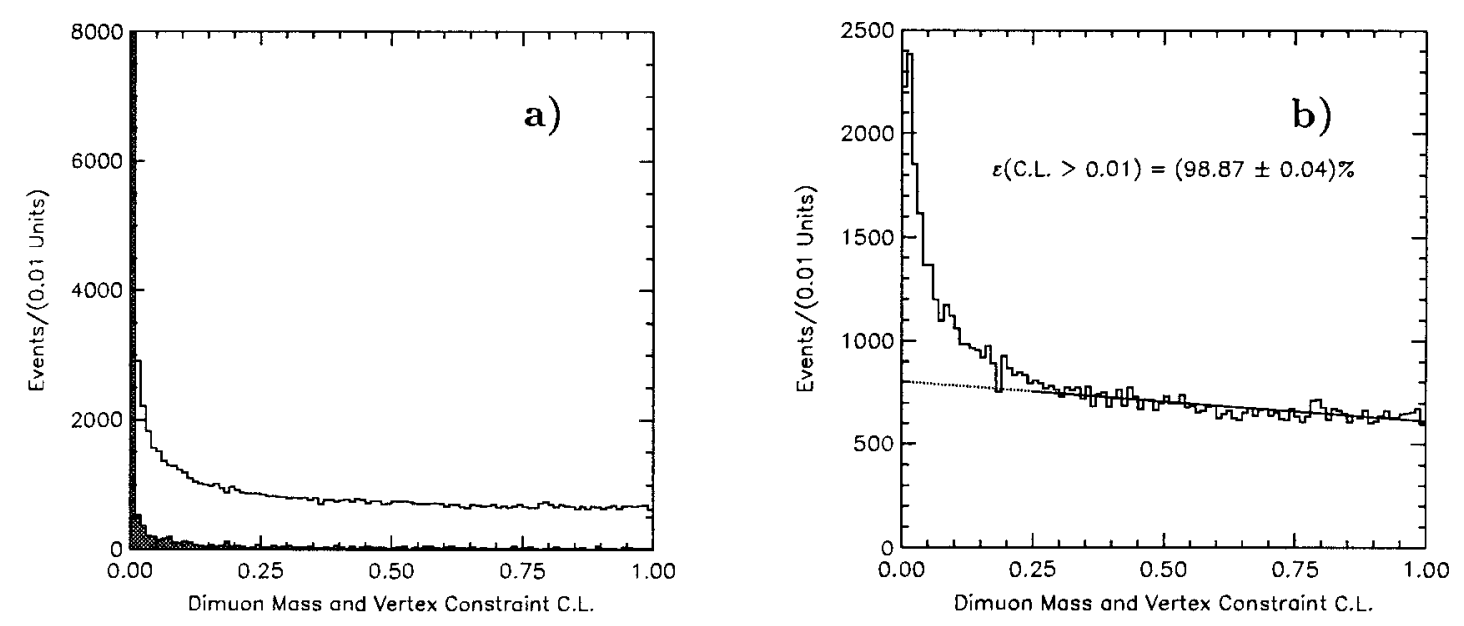

Figure 3.8: The confidence level distribution of the dimuon mass and vertex fit. The shaded region represents the confidence level of the fit for the same charge dimuons. Figures (a) and (b) represent the distributions before and after a background subtraction is made. The efficiency is obtained by taking the ratio of the integrated line between 0.01 and 1.0 and the entire range.

distribution to the low end. The fitted line results in a measured efficiency that agrees with the cut value; the actual efficiency is lower when one considers the asymmetric mass distribution. This asymmetry causes the excess of events in the lower region of the confidence level distribution shown in Figure 3.8 (b). There are approximately 1500 events in the lowest bin above the nominal value which will be removed with the C.L. $\left(\chi^{2}\right)>0.01$ requirement. This cut therefore has an inefficiency of $2 \%$.

The efficiency of the cut can also be extracted from Figures 3.4 (b) and 3.6 (b). A $\left(C . L .\left(\chi^{2}\right)>0.01\right)$ requirement corresponds to the area within 2.6 standard deviations of the normalized mass distributions. Using Figures 3.4 (b) and 3.6 (b), the requirement that the normalized masses be within 2.6 standard deviations of zero gives a mean efficiency of $(97.5 \pm 0.4) \%$, where the uncertainty is taken to be half of the difference in efficiency given by these two techniques.

These studies confirm that the inefficiencies are not large (though the actual value is not important in this analysis) and the background rejection is significant.

The remaining backgrounds under the $J / \psi$ resonance are expected to be dominated by fake muons and punch-through particles (hadrons that manage to pass through the calorimeters). 

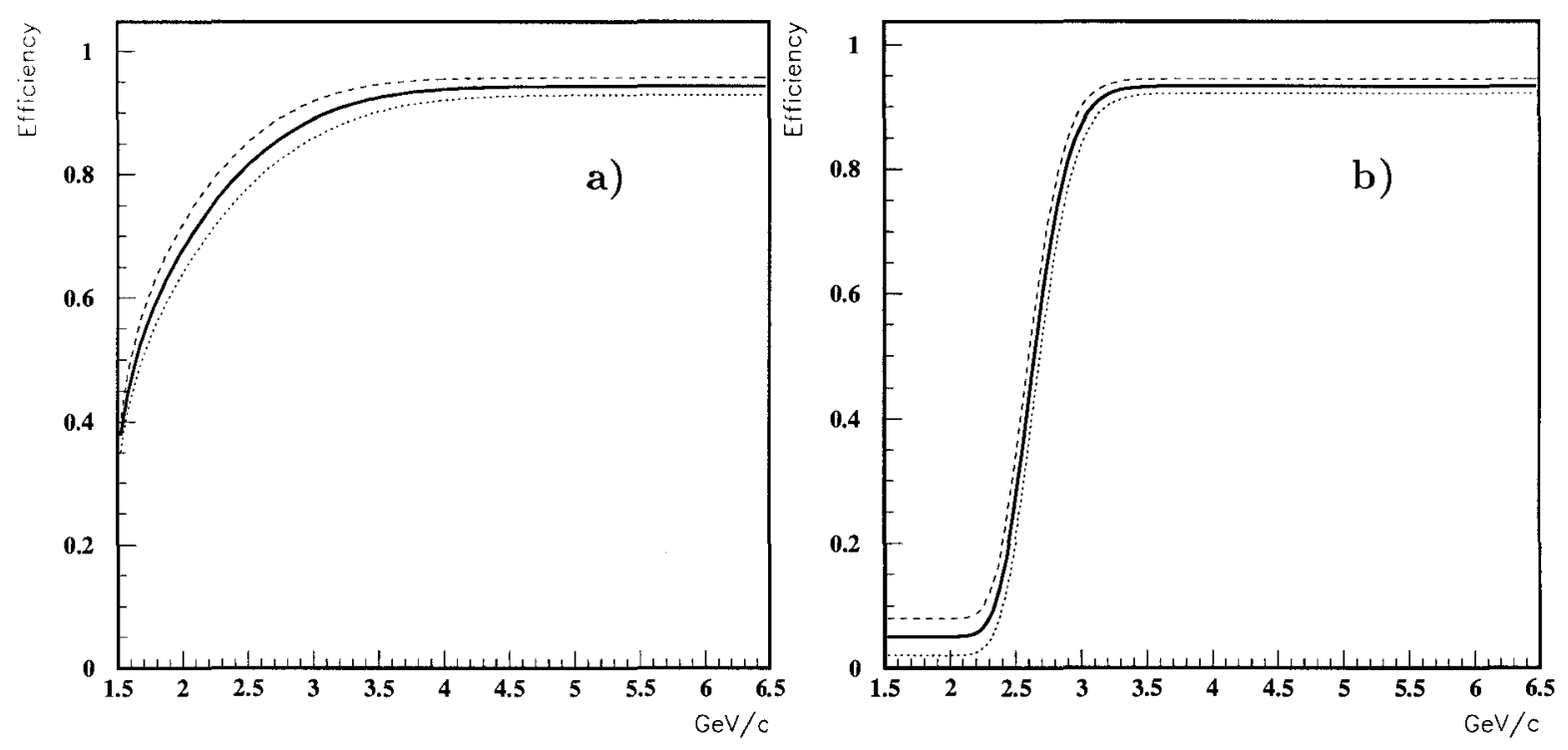

Figure 3.9: The level 1 and level 2 low $P_{T}$ central muon trigger efficiency curves. Figures (a) and (b) represent the distributions for the level 1 and 2 muon efficiency as a function of $P_{T}$. The higher dashed and lower dotted curves are one standard deviations of the trigger parameterizations.

Two additional requirements were made on the muon candidates to remove some of the remaining backgrounds. Of the two muons, the muon with the smaller transverse momentum was required to have a transverse momentum greater than $1.8 \mathrm{GeV} / c$ and the other muon was required to have a transverse momentum greater than $2.5 \mathrm{GeV} / c$. These cuts ensure that the muon candidates are in a regime of high level 1 and level 2 trigger efficiency. The level 1 and level 2 muon efficiencies as a function of muon transverse momentum are shown in Figure 3.9 [55]. The actual chosen cut value somewhat arbitrary. The values of $1.8 \mathrm{GeV} / c$ and $2.5 \mathrm{GeV} / c$ were used because they represent the $60 \%$ and $30 \%$ ( $25 \%$ higher than the respective minimum efficiency) level of the respective level 1 and level 2 trigger curves. The level $3 \mathrm{~J} / \psi$ efficiency is independent of muon momentum, and is $(97 \pm 2) \%$. It should be noted that these two momentum requirements were applied after all the tracks from the different bottom mesons candidates were fitted with the appropriate constraints. This requirement will be discussed in later sections of this chapter. 


\subsection{Strange Meson Requirements}

As was the case for the $J / \psi$ candidates, all of the daughters of the strange meson candidates were required to have the correct charge assignment. Each candidate track was assigned the mass corresponding to the respective decay hypothesis. This was done because kaons and pions cannot be distinguished from each other using the CDF detector. This inability to identify which tracks are kaons or pions leads to large combinatorial backgrounds. In order to reduce these backgrounds, each meson candidate was also required to have a transverse momentum above a certain value.

The strange meson transverse momentum requirement was chosen by examining various $P_{T}$ cuts in intervals of $0.5 \mathrm{GeV} / c$ for all of the five decays of interest. The values were chosen to maximize the significance $(S / \sqrt{S+B})$ of the five decays, where $\mathrm{S}$ is the number of signal events taken from Monte Carlo calculations and $\mathrm{B}$ is the number of background events in the signal region taken from data. A similar requirement was also made for the bottom meson candidates. This requirement will be discussed further in the next section.

For the strange mesons that decay strongly $\left(K^{*}(892)^{+}, K^{*}(892)^{0}\right.$ and $\left.\phi(1020)\right)$, further background rejection was accomplished by requiring that the invariant mass of the strange mesons lie within a specific range. The respective mass ranges of \pm 80 $\mathrm{MeV} / c^{2}, \pm 80 \mathrm{MeV} / c^{2}$ and $\pm 10 \mathrm{MeV} / c^{2}$ for the $K^{*}(892)^{+}, K^{*}(892)^{0}$ and $\phi(1020)$, respectively, are large enough to be highly efficient while significantly reducing the combinatoric background. In principle, a mass window cut around the center of the resonance within two natural widths $(2 \Gamma)$ would be $84.4 \%$ efficient if the resonance has a Breit-Wigner line shape. Taking the PDG [7] values, a two width mass window cut would then be about $\pm 9 \mathrm{MeV} / c^{2}$ and $\pm 100 \mathrm{MeV} / c^{2}$ for the $\phi(1020)$ and $K^{*}(892)$ decays, respectively. $\mathrm{A} \pm 100 \mathrm{MeV} / c^{2}$ cut on the $K^{*}(892)$ invariant mass is not optimal due to the larger combinatorial backgrounds. This can be seen from Figure 3.10 which shows the invariant mass spectra of inclusive $K^{*}(892)^{+}$and $K^{*}(892)^{0}$ decays. To further reduce backgrounds for the $K^{*}(892)$ decays, the $80 \%$ efficiency mark, or $\pm 77 \mathrm{MeV} / \mathrm{c}^{2}$, would be a better choice. Thus, the nearest decade values of $\pm 10 \mathrm{MeV} / \mathrm{c}^{2}$ and $\pm 80 \mathrm{MeV} / c^{2}$ for the $\phi(1020)$ and $K^{*}(892)$ decays were chosen, respectively.

An interesting thing to note is that with enough statistics, one can overcome the problems due to the large $\bar{p} p$ cross section and lack of particle identification and 

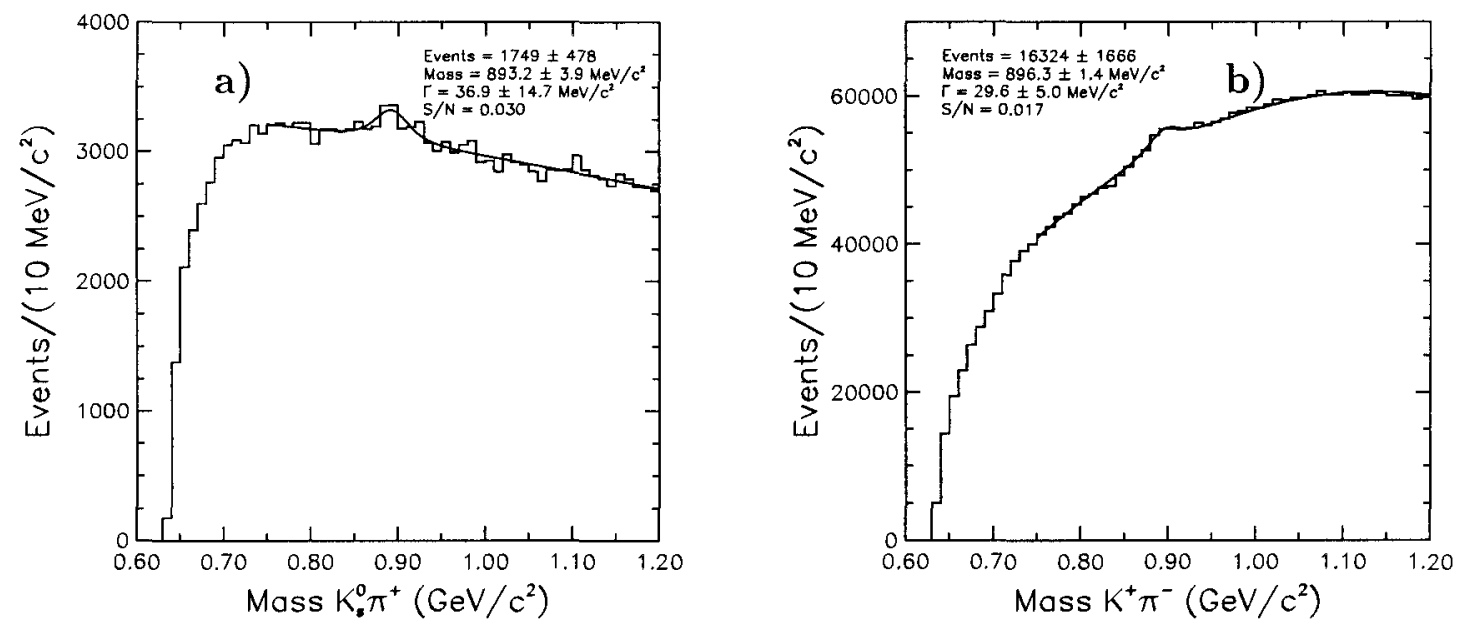

Figure 3.10: Invariant mass distribution of (a) $K_{s}^{0} \pi^{+}$and (b) $K^{+} \pi^{-}$pairs. The resonances were fitted to a Breit-Wigner line shape convoluted with Gaussian errors. The background shape were taken to be a second-order polynomial. The signal to noise value was calculated in the mass window cut region. The neutral strange mesons were vertex constrained and had a minimum transverse momentum requirement of $2 \mathrm{GeV} / c$. The mass of $K_{s}^{0}$ meson from the $K^{*}(892)^{+}$was constrained to the world average value.

observe inclusive particle resonances with large natural widths. For example, the inclusive $K^{*}(892)^{0}, K^{*}(892)^{+}$and $\phi(1020)$ resonances are all clearly observable, as is illustrated in Figures 3.10 and 3.11

\subsection{Bottom Meson Requirements}

The values of the momentum cuts on the strange as well as on the bottom mesons were chosen after examining the effect of the momentum cuts on various quantities of interest using three different methods. One method involved calculating the significance of the bottom meson resonance signal as a function of the momentum cuts on the strange and bottom mesons. The momentum cut values were chosen in intervals of $1 \mathrm{GeV} / c$ units for the bottom mesons and $0.5 \mathrm{GeV} / c$ units for the strange mesons. For this method, the objective was to maximize the significance $(S / \sqrt{\mathrm{S}+\mathrm{B}})$ with $\mathrm{S}$ being the number of events taken from a Monte Carlo calculation, and $\mathrm{B}$ being the number of background events under the signal region taken from data. The signal 


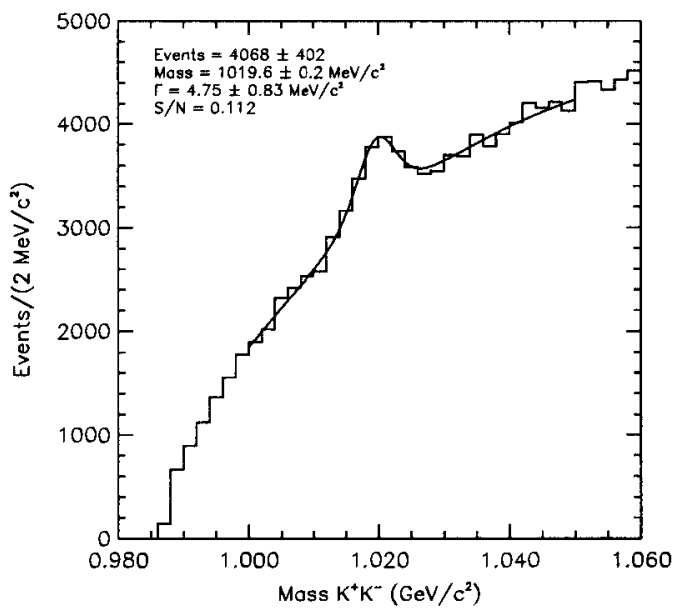

Figure 3.11: Invariant mass distribution of $K^{+} K^{-}$pairs. The resonance was fitted to a Breit-Wigner line shape convoluted with Gaussian errors. The background shape was taken to be a second-order polynomial. The signal to noise value was calculated in the mass window cut region. The $\phi(1020)$ meson was vertex constrained and had a minimum transverse momentum requirement of $2 \mathrm{GeV} / c$.

region was taken to be within three standard deviations of the nominal mass of the bottom meson resonance. The number of Monte Carlo signal events was normalized to give the expected number of decays for the five channels with a minimum transverse momentum of $10 \mathrm{GeV} / c$. The second method is the same as the first with both $\mathrm{S}$ and $\mathrm{B}$ determined from the data events. The third method is the same as the second one with the exception that what is maximized is the sum of the significance of the five decays. The momentum requirements that maximized the significance for the three methods are listed in Table 3.3. The first and second methods give the same results with the exception of the bottom meson transverse momentum requirement for the $B^{+} \rightarrow J / \psi K^{+}$decay. Because the second method is in principle the most sensitive to statistical fluctuations, it is not used. The first and third methods are reasonable, with each having some advantages and disadvantages. As will be shown later, method 1 gives smaller statistical uncertainties while method 3 gives a more unbiased estimate of the number of events and efficiencies. To determine which method is best, both the first (different momentum requirements) and third (same momentum requirements) criteria are considered. The criteria that gives the minimum combined statistical and systematic uncertainties on the ratios of branching fractions is then considered the 


\begin{tabular}{|l|c|c|c|}
\hline \hline Decay & Method 1 & Method 2 & Method 3 \\
\hline \hline$B^{+} \rightarrow J / \psi K^{+}$ & $1.5,8.0$ & $1.5,6.0$ & $2.0,8.0$ \\
\hline$B^{+} \rightarrow J / \psi K^{*}(892)^{+}$ & $2.0,6.0$ & $2.0,6.0$ & $2.0,8.0$ \\
\hline$B^{0} \rightarrow J / \psi K^{0}$ & $1.5,6.0$ & $1.5,6.0$ & $2.0,8.0$ \\
\hline$B^{0} \rightarrow J / \psi K^{*}(892)^{0}$ & $2.0,8.0$ & $2.0,8.0$ & $2.0,8.0$ \\
\hline$B_{s}^{0} \rightarrow J / \psi \phi(1020)$ & $2.0,6.0$ & $2.0,6.0$ & $2.0,8.0$ \\
\hline
\end{tabular}

Table 3.3: Optimum transverse momentum (in $\mathrm{GeV} / c$ ) cut values for the strange and bottom mesons based on different criteria.

best. Thus, both set of requirements are considered in the calculation of the ratios of branching fractions.

As for the $J / \psi$ candidates, the backgrounds to the bottom meson decays were further reduced with additional vertex constraints on the charged tracks from the bottom meson decay. Decays that involved $K_{s}^{0}$ mesons had additional requirements. The dipion candidates were vertex constrained and their invariant mass was constrained to the world average $K^{0}$ mass of $497.672 \mathrm{MeV} / c^{2}$ [7]. The resulting confidence level of the fit was required to be greater than $1 \%$.

The effects of the additional dipion vertex and $K_{s}^{0}$ mass constraints for the $B^{+} \rightarrow$ $J / \psi K^{*}(892)^{+}$and $B^{0} \rightarrow J / \psi K^{0}$ decays were studied using the same methods that were used for the inclusive $J / \psi$ study. The efficiencies for the dipion component of the $B$ meson confidence level requirements involving $K_{s}^{0}$ mesons were then taken to be the same for those determined with the use of the inclusive $K_{s}^{0}$ background subtracted mass, normalized mass and confidence level distributions. As for the inclusive $J / \psi$ efficiencies, half of the efficiencies were obtained by fitting the respective spectra with the background shape taken from same charge events. The ratio of opposite charge to same charge events, in principle should be $\mathrm{N} /(\mathrm{N}-2)$, where $\mathrm{N}$ is the average number of charged tracks per event. The fitted normalization value from Figure 3.14 (a) was 1.10 , which implies that the average number of charged tracks should be 22 for a a typical $K_{s}^{0}$ decay. This is somewhat less than the actual value, which is in the low thirties as will be shown later. One explanation of this feature is that the reconstruction efficiency for two charged tracks curving in the same direction in the CTC is slightly worse.

Using Figures 3.12 to 3.16 , the mean efficiency for the dipion vertex and $K_{s}^{0}$ mass 

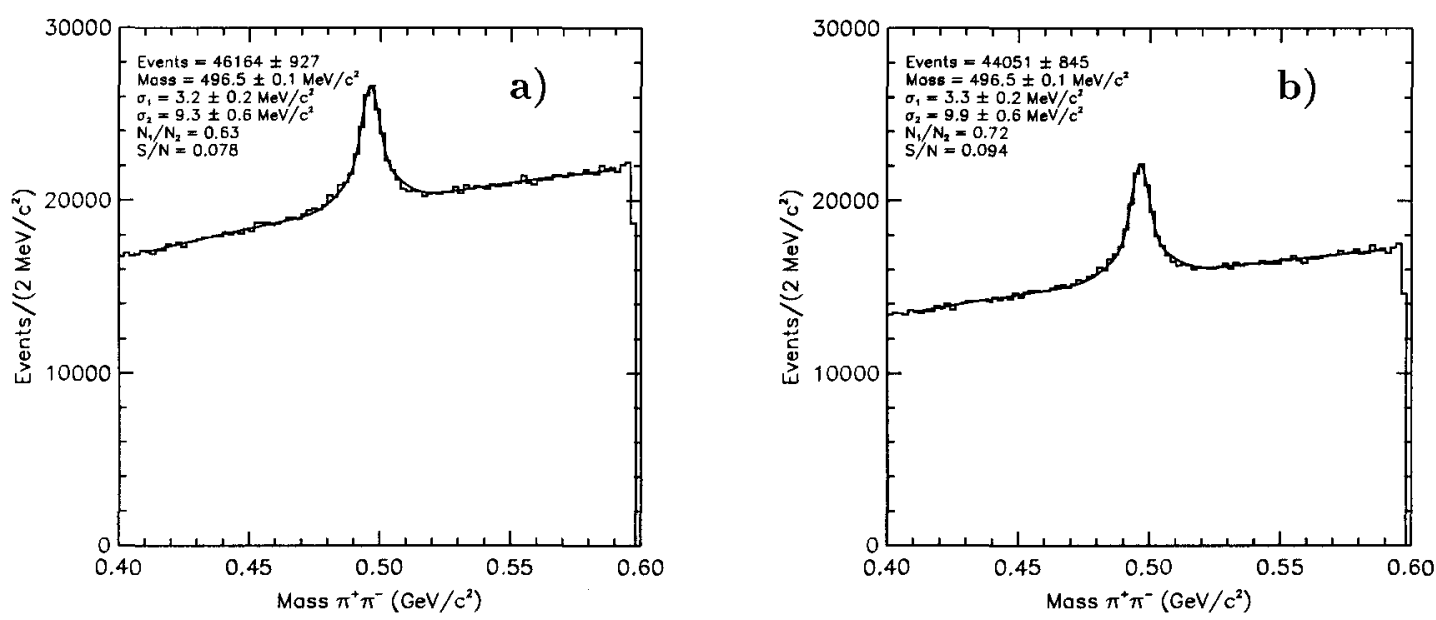

Figure 3.12: Invariant mass distribution of $\pi^{+} \pi^{-}$pairs (a) before and (b) after the confidence level requirement is applied when the dipions are vertex constrained. The spectra were fitted to a double Gaussian while the background was taken to be a second-order polynominal.

constrained confidence level requirement using the five methods was determined to be $(94.3 \pm 3.0) \%$, where the uncertainty is the standard deviation of the five different values of the efficiency. Using only the first four methods, this efficiency becomes $(93.1 \pm 1.7) \%$.

The efficiency of the dipion vertex and $K_{s}^{0}$ mass constraint C.L. $\left(\chi^{2}\right)>0.01$ cut was also obtained in the same manner as the dimuon vertex and $J / \psi$ mass constraint confidence level requirement. The number of events that pass this cut was estimated by integrating the fitted Gaussian distribution that parameterized the normalized invariant mass resonance within \pm 2.6 standard deviations around zero. This \pm 2.6 standard deviation cut on the resonances from Figures 3.13 and 3.15 resulted in a mean efficiency of $(97.4 \pm 0.3) \%$, where the uncertainty was taken to be half the difference between the two values.

The efficiency of the dipion vertex and $K_{s}^{0}$ mass constraint C.L. $\left(\chi^{2}\right)>0.01$ cut was also obtained by fitting the lifetime distributions of the dipions before and after the requirement was made. Using the number of events from the lifetime distributions shown in Figures 3.18 and 3.19 gives a mean efficiency of $(97.9 \pm 0.1) \%$, where the uncertainty is statistical. The actual efficiency is probably somewhere in between the two calculated values $(97.7 \pm 0.3) \%$. The uncertainty is taken to be half the 

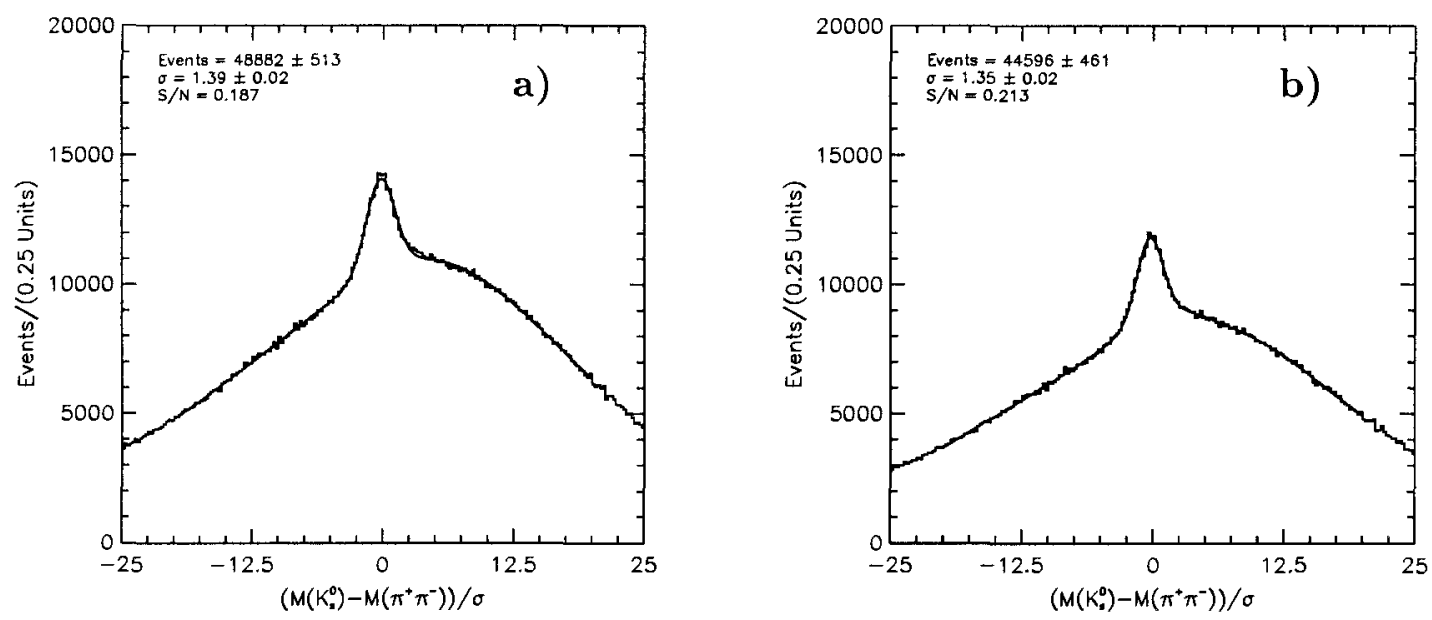

Figure 3.13: Normalized invariant mass distribution of $\pi^{+} \pi^{-}$pairs (a) before and (b) after the confidence level requirement is applied when the dipions are vertex constrained. The spectra were fitted to a Gaussian while the background was taken to be a Gaussian on the high end and a second-order polynomial on the low end. The asymmetry of the background is a result of phase space effects.
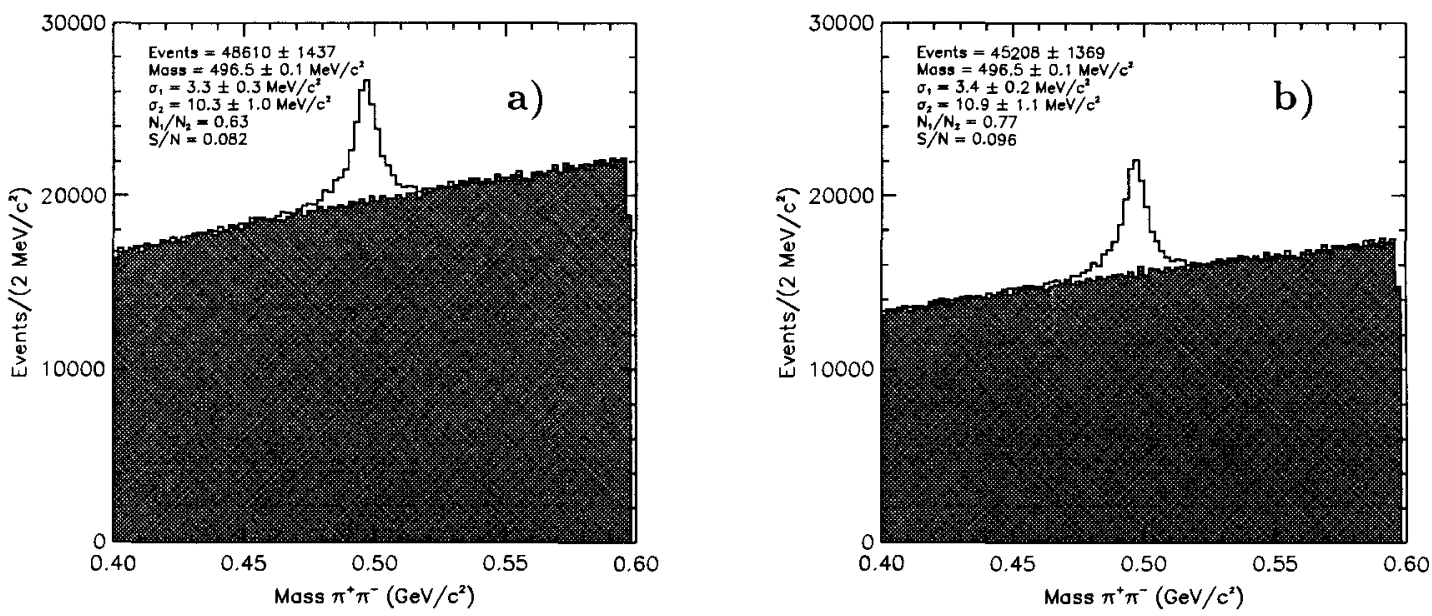

Figure 3.14: Invariant mass distribution of $\pi^{+} \pi^{-}$pairs (a) before and (b) after the confidence level requirement is applied when the dipions are vertex constrained. The shaded region represents the invariant mass distribution for the same charge pion pairs. The spectra were fitted to a double Gaussian and the background was normalized to the $\pi^{ \pm} \pi^{ \pm}$invariant mass distribution. 

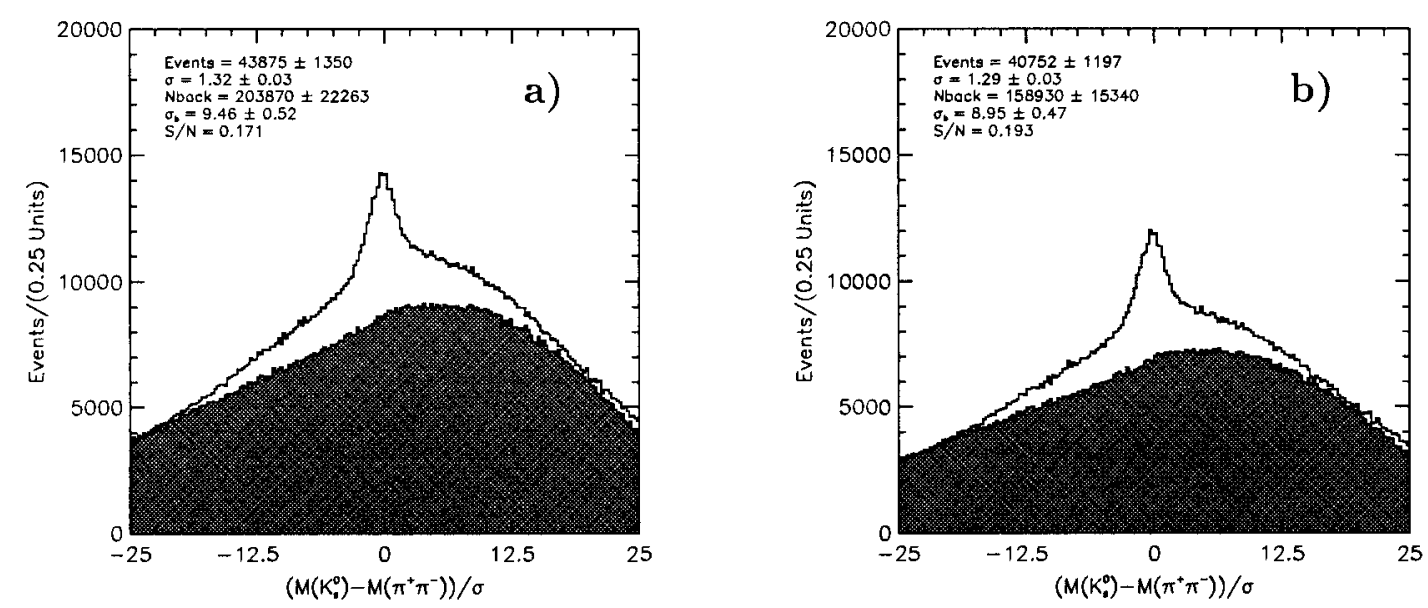

Figure 3.15: Normalized invariant mass distribution of $\pi^{+} \pi^{-}$pairs (a) before and (b) after the confidence level requirement is applied when the dipions are vertex constrained. The shaded region represents the normalized invariant mass distribution for the same charge pion pairs. The spectra were fitted to a double Gaussian and the background was normalized to the $\pi^{ \pm} \pi^{ \pm}$normalized invariant mass distribution with an extra Gaussian term. The asymmetry of the background is a result of phase space effects.
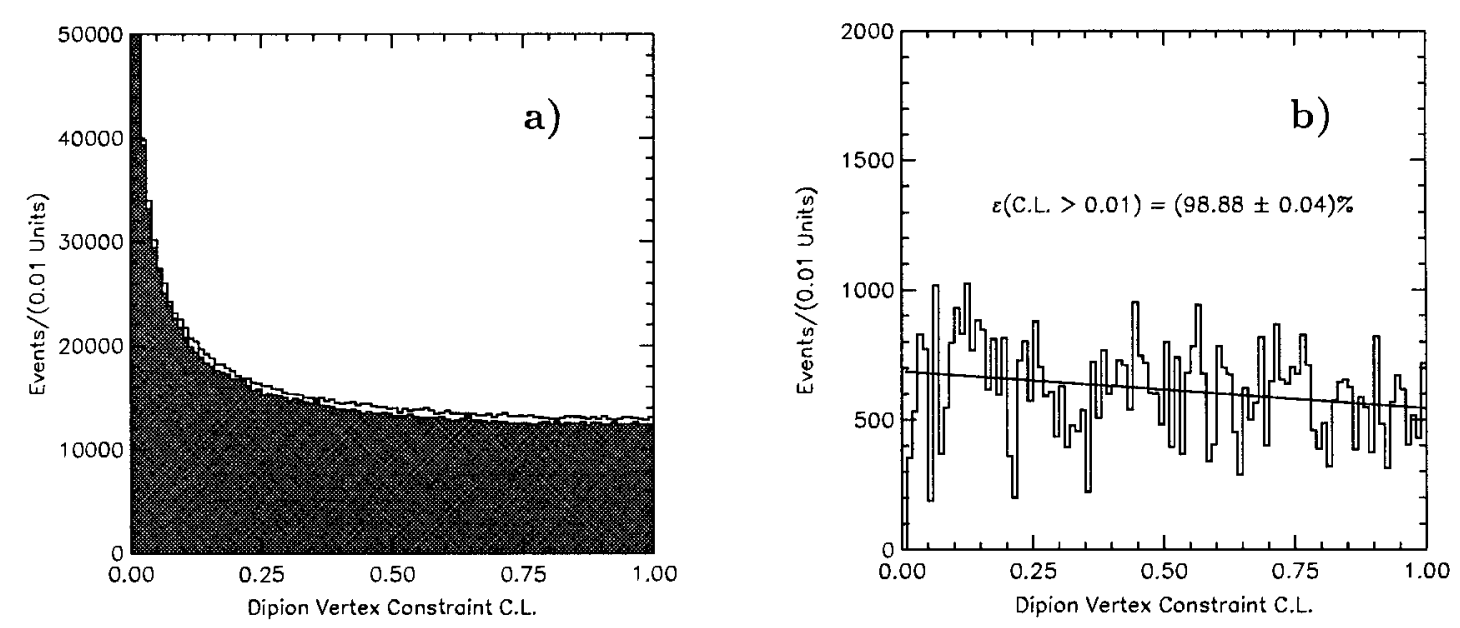

Figure 3.16: The confidence level distribution of the dipion vertex fit. The shaded region represents the confidence level of the fit for the same charge dipions. Figures (a) and (b) represent the distributions before and after a background subtraction is made. 

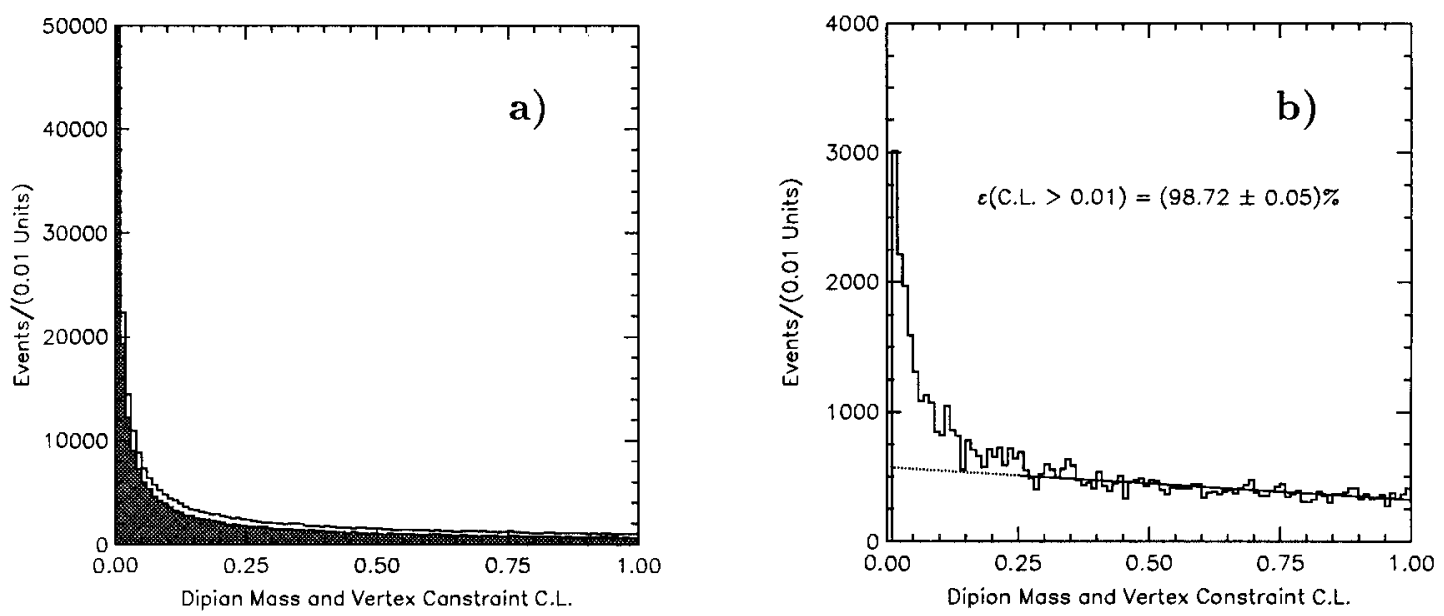

Figure 3.17: The confidence level distribution of the dipion mass and vertex fit. The shaded region represents the confidence level of the fit for the same charge dipions. Figures (a) and (b) represent the distributions before and after a background subtraction is made.

difference.

The dikaon component of the vertex constraint confidence level requirement for the $B_{s}^{0} \rightarrow J / \psi \phi(1020)$ decay was determined with fewer methods. The efficiency was not estimated with the use of the normalized mass spectra because of the noticeable natural width of the $\phi(1020)$ meson. The lifetime spectra were not used because of the very small lifetime of the $\phi(1020)$ meson. However, the efficiency of the dikaon vertex constraint confidence level requirement can be obtained by taking the ratio of fitted events from the mass spectra before and after the cut was applied. Using the inclusive $\phi(1020)$ invariant mass resonance, the efficiency of the C.L. requirement was then determined to be $(96.5 \pm 0.3) \%$.

The same charge dikaon mass distribution, shown in Figure 3.21, has a different shape than the combinatorial background in the opposite charge sample. This is due in part to the proximity of the mass of the $\phi(1020)$ meson to the mass of two kaons. Therefore, the same charge dikaon mass distribution was not used to determine the efficiency of the confidence level requirement.

Since the efficiencies for the individual dimuon, dipion and dikaon confidence level requirements are very high, it is expected that a combined vertex constraint confidence level requirement on the charged tracks from the bottom mesons decays will also be 

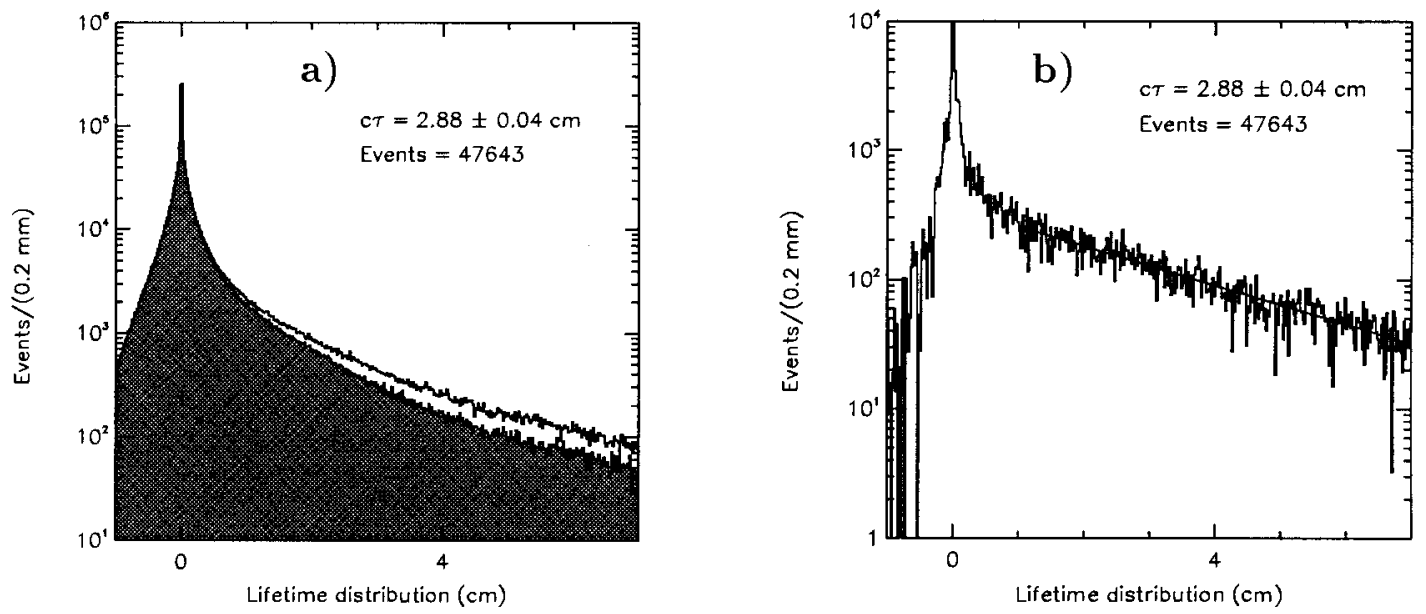

Figure 3.18: The lifetime distribution of the dipion events that have been vertex constrained with the confidence level cut applied. The shaded region represents the lifetime distribution of the fit for the same charge dipions. Figures (a) and (b) represent the distributions before and after a background subtraction is made.
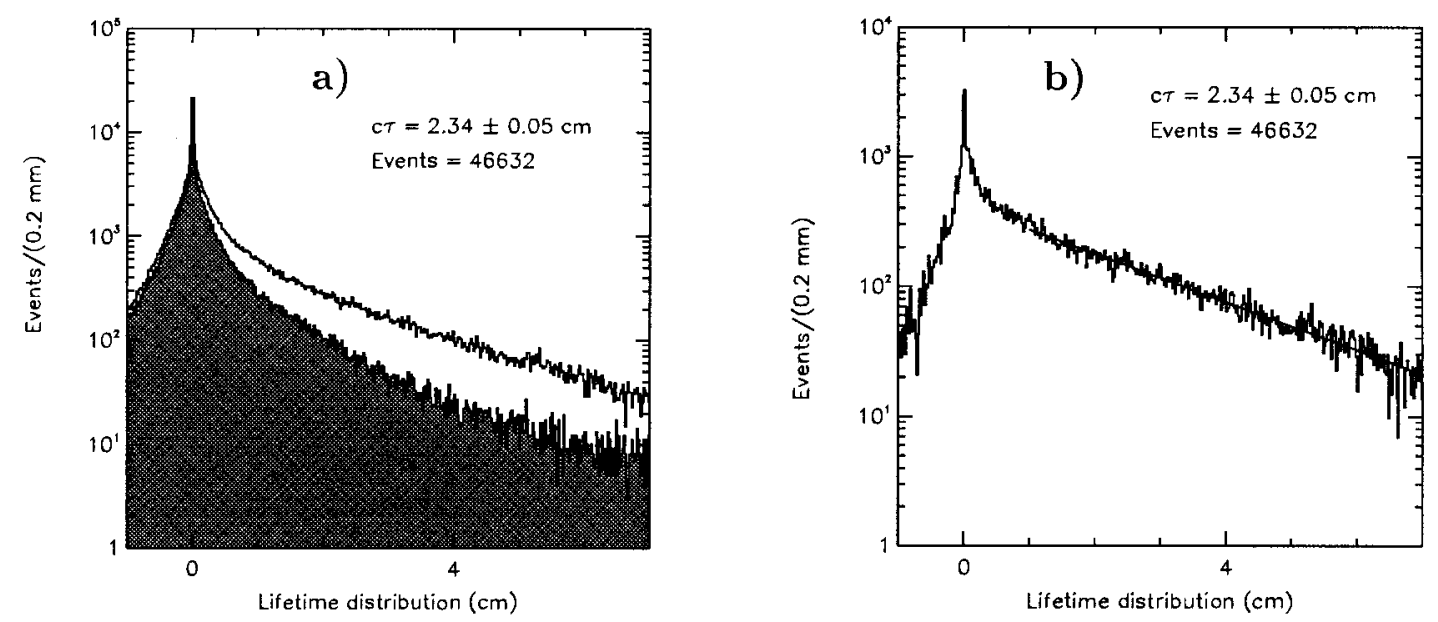

Figure 3.19: The lifetime distribution of the dipion events that have been vertex and mass constrained with the confidence level cut applied. The shaded region represents the lifetime distribution of the fit for the same charge dipions. Figures (a) and (b) represent the distributions before and after a background subtraction is made. 

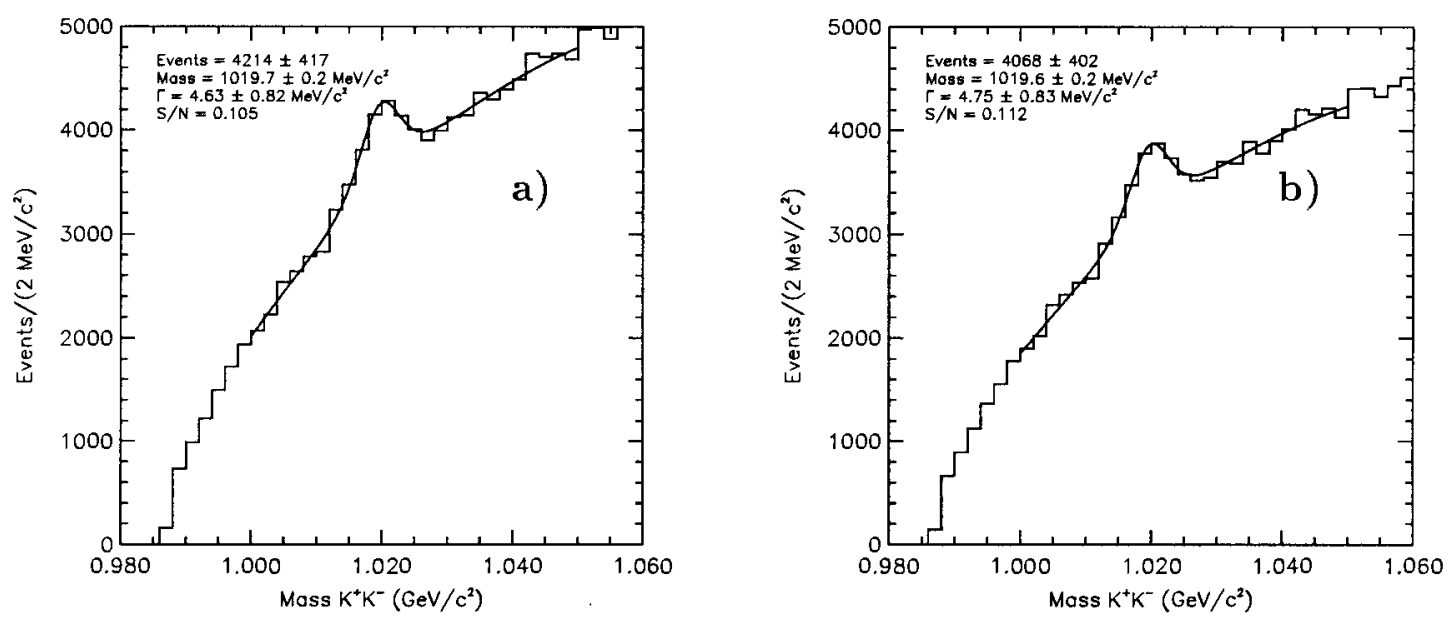

Figure 3.20: Invariant mass distribution of $K^{+} K^{-}$pairs (a) before and (b) after the confidence level requirement is applied when the dikaons are vertex constrained. The spectra were fitted to a Breit-Wigner convoluted with Gaussian errors. The background was taken to be a second-order polynomial.

highly efficient.

Half of the remaining background for reconstructed bottom mesons was removed with the additional requirement that the candidate bottom meson flight path be pointing in the same hemisphere as its momentum vector. This requirement is in effect requiring that the candidate bottom mesons have a positive proper decay length $(c \tau)$ which is defined by

$$
c \tau \equiv \frac{M}{P_{T}^{2}}\left(\overrightarrow{X_{T}} \cdot \overrightarrow{P_{T}}\right)
$$

where $\mathrm{M}$ is the mass of the parent particle, $\overrightarrow{P_{T}}$ is the transverse momentum vector of the parent particle and $\overrightarrow{X_{T}}$ is the transverse vector pointing from the primary vertex to the secondary $(B)$ vertex.

Because the lifetime of $K_{s}^{0}$ mesons is much greater than bottom mesons, a larger proper decay length requirement was applied on the dipion decay vertex with respect to the candidate bottom meson decay vertex. The proper decay length distributions for the $K_{s}^{0}$ candidates are shown in Figures 3.18 and 3.19. A value of $1 \mathrm{~mm}$ was chosen for the $c \tau$ cut to remove the large background from prompt candidates while at the same time being highly efficient. The efficiency of this cut would be $96.3 \%$ if 

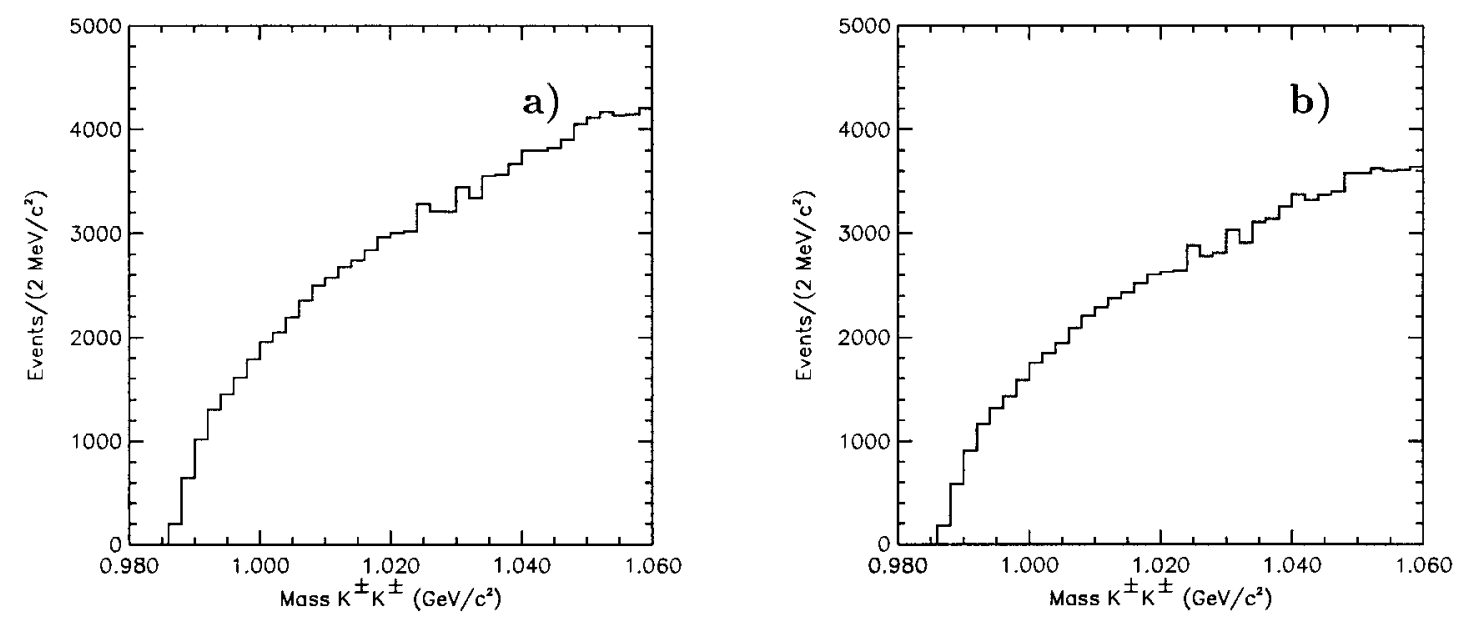

Figure 3.21: Invariant mass distribution of $K^{ \pm} K^{ \pm}$pairs (a) before and (b) after the confidence level requirement is applied when the dikaons are vertex constrained.
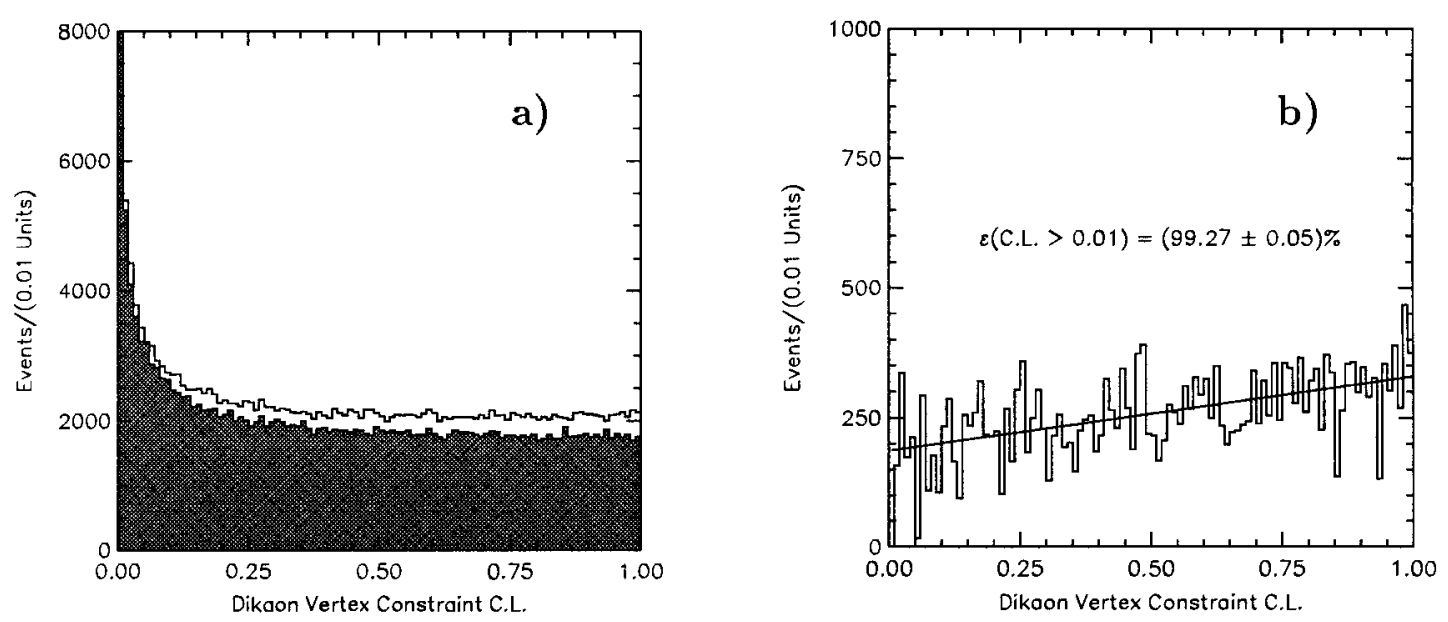

Figure 3.22: The confidence level distribution of the dikaon vertex fit. The shaded region represents the confidence level of the fit for the same charge dikaons. Figures (a) and (b) represent the distributions before and after a background subtraction is made. 
one assumes the world average proper decay length value of $2.676 \mathrm{~cm}$ [7]. However, within the statistical uncertainties, the fitted proper decay length of the $K_{s}^{0}$ mesons is inconsistent with the world averages. This inconsistency is a result of not accounting for non- $K_{s}^{0}$ decays into opposite charged tracks that have large proper decay lengths. Nevertheless, a $1 \mathrm{~mm}$ requirement on the dipions should be very efficient while at the same time removing many of the background events.

\subsection{Primary Vertex Considerations}

In order to make a $c \tau$ cut on the $B$ meson candidates, one must first know the location of the primary $\bar{p} p$ vertex. There are several ways to determine this. One method involves determining the transverse position of the primary vertex and the associated uncertainties on an event to event basis. An alternative method involves using the average transverse vertex position of the beam line determined from many events. The uncertainties on the transverse position of the primary vertex can also be fixed to average values. The longitudinal position of the $\bar{p} p$ vertex is always calculated on an event to event basis. Unlike the transverse position, the longitudinal position changes noticeably from event to event.

In addition, one can constrain the candidate $B$ or $K_{s}^{0}$ meson flight path to be parallel to its momentum vector in either three dimensions (3-D pointing constraint) or in the transverse plane (2-D pointing constraint).

To determine the best method for evaluating the primary $\bar{p} p$ vertex position and the best pointing requirement a study of four quantities with the various requirements was performed. The four quantities that were examined are: number of reconstructed $B$ candidates, standard deviation of the $B$ invariant mass distributions, signal to noise of the $B$ resonance, and significance of the signal (fitted number of events/uncertainty in the fitted number). The best method would then be the method that simultaneously maximizes the number of reconstructed $B$ candidates, the signal to noise of the $B$ resonance, and the significance of the signal while minimizing the standard deviation of the $B$ invariant mass distributions. The four quantities are summarized in the tables given in Appendix A with similar cuts that have been discussed up to this point. From the various tables in Appendix A, one can see that there is no one set of requirements that can be applied to satisfy the best method criteria for all the five decays. In addition, several one standard deviation variations are seen in the four 
variables, so making conclusive statements is difficult.

Since the ratios of branching fractions are obtained in part from the ratios of numbers of reconstructed bottom mesons, the most important quantity to examine is the significance of the signals. One observation concerning the significance for the decays that involve long lived particles such as a $K_{s}^{0}$ meson is that it is best to make only a 3-D pointing requirement. This can be seen when one compares rows 2 and 3 for columns 1 to 3 of Tables A.19 to A.24. Going to an additional pointing requirement (columns 4 to 5 ) does not seem to offer significant improvement. So for decays involving a long lived particle $\left(K_{s}^{0}\right)$, only a 3-D pointing requirement was used. For decays that do not have a long lived particle (rows 1, 4, and 5), a pointing requirement seems to be on average a better choice. Since the uncertainty on the longitudinal position of the primary vertex is large, the 2-D pointing requirement was used instead of the 3-D pointing requirement.

Since the sum of the significance for the five decays with the intermediate $P_{T}$ values and 3-D constraints was larger for the run-averaged beam position, it was chosen as the default.

In summary, one pointing requirement was made on all five decays of interest. For decays involving a $K_{s}^{0}$ meson, a $3-\mathrm{D}$ pointing requirement was made to the $B$ vertex. For the other decays, a $2-\mathrm{D}$ pointing requirement was made to the primary vertex. The transverse primary vertex was taken from a run averaged measurement of the $\bar{p} p$ collision point. The uncertainties on the transverse distances were fixed at $40 \mu \mathrm{m}$, which is the average standard deviation of the transverse position of the primary vertex.

\subsection{Reconstruction of Bottom Mesons}

With the use of the requirements described in the previous sections one is able to observe clear resonances in all of the five decays. A summary of the requirements that were used is listed below.

- Track quality requirements

1. $P_{T}>400 \mathrm{MeV} / c$.

2. Tracks must have at least 4 hits in at least 2 CTC axial super layers.

3. Tracks must have at least 2 hits in at least 2 CTC stereo super layers. 
4. Tracks must traverse the entire radius of the CTC tracking volume.

5. SVX-CTC fit is used if $\chi^{2}(S V X) / N d o f<6.0$ and the track has at least 3 SVX hits.

- $J / \psi$ and Muon Cuts

1. Correct muon charge assignment.

2. Minimum muon $P_{T}>1.4 \mathrm{GeV} / c$.

3. CTC - muon $(r-\phi)$ and $z$ matching.

4. Dimuon vertex and mass constrained C.L. $\left(\chi^{2}\right)>0.01$.

5. $\operatorname{Min}\left(P_{T}^{\mu^{+}}, P_{T}^{\mu^{-}}\right)>1.8 \mathrm{GeV} / c$ after all constraints.

6. $\operatorname{Max}\left(P_{T}^{\mu^{+}}, P_{T}^{\mu^{-}}\right)>2.5 \mathrm{GeV} / c$ after all constraints.

- Strange Meson Requirements

1. Correct track charge assignment.

2. $P_{T}>1.5$ or $2 \mathrm{GeV} / c$.

3. $\left|M\left(K^{+} K^{-}\right)-M(\phi(1020))_{P D G}\right|<10 \mathrm{MeV} / c^{2}$.

4. $\left|M(K \pi)-M\left(K^{*}(892)\right)_{P D G}\right|<80 \mathrm{MeV} / c^{2}$.

- Bottom Meson Requirements

1. $P_{T}>6$ or $8 \mathrm{GeV} / c$.

2. Vertex and $J / \psi$ mass constrained (and $K_{s}^{0}$ vertex and mass constrained) C.L. $\left(\chi_{B}^{2}\right)>0.01$.

3. $c \tau(B)>0,\left(c \tau\left(K_{s}^{0}\right)>1 \mathrm{~mm}\right)$.

The candidate $B$ meson invariant mass spectra after these cuts were applied are shown in Figures 3.23 to 3.27 .

\subsubsection{Number of Observed Decays}

The number of observed decays can be obtained by fitting the spectra shown in Figures 3.23 to 3.27 with the expected line shapes. For four of the decays this amounts to fitting the spectra with one Gaussian representing the signal region and a linear term 


\begin{tabular}{|l|c|}
\hline \hline Decay & Number of Events \\
\hline \hline$B^{+} \rightarrow J / \psi K^{+}$ & $153.5 \pm 18.9$ \\
\hline$B^{+} \rightarrow J / \psi K^{*}(892)^{+}$ & $12.9 \pm 4.3$ \\
\hline$B^{0} \rightarrow J / \psi K_{s}^{0}$ & $36.9 \pm 7.3$ \\
\hline$B^{0} \rightarrow J / \psi K^{*}(892)^{0}$ & $95.5 \pm 14.3$ \\
\hline$B_{s}^{0} \rightarrow J / \psi \phi(1020)$ & $29.4 \pm 6.2$ \\
\hline \hline
\end{tabular}

Table 3.4: Number of reconstructed $B$ meson decays using different momentum cuts.

\begin{tabular}{|l|c|}
\hline \hline Decay & Number of Events \\
\hline \hline$B^{+} \rightarrow J / \psi K^{+}$ & $123.3 \pm 15.4$ \\
\hline$B^{+} \rightarrow J / \psi K^{*}(892)^{+}$ & $12.6 \pm 4.2$ \\
\hline$B^{0} \rightarrow J / \psi K_{s}^{0}$ & $25.5 \pm 5.7$ \\
\hline$B^{0} \rightarrow J / \psi K^{*}(892)^{0}$ & $95.5 \pm 14.3$ \\
\hline$B_{s}^{0} \rightarrow J / \psi \phi(1020)$ & $25.8 \pm 5.7$ \\
\hline \hline
\end{tabular}

Table 3.5: Number of reconstructed $B$ mesons decays using similar momentum cuts.

representing the background. For the $B^{0} \rightarrow J / \psi K^{*}(892)^{0}$ decay, the signal is parameterized by two Gaussian line shapes. This decay has to be treated differently since both mass combinations of the $K^{*}(892)^{0}$ daughters can contribute to the resonance. This problem is due to the inability to determine which track was the kaon or pion. The amplitude and standard deviation of the second Gaussian distribution was scaled to the amplitude and standard deviation of the first Gaussian representing the signal. These scale factors were obtained via a Monte Carlo calculation: the amplitude scale factor is 0.08 and the standard deviation scale factor is 3.3. The center of the second Gaussian was also shifted lower by $2.3 \mathrm{MeV} / c^{2}$. The resulting fitted number of events for each decay using the two groups of transverse momentum cuts are summarized in Tables 3.4 and 3.5 .

The number of events that are shown in Tables 3.4 and 3.5 is based on certain assumptions. For three of the five decays that involve strongly decaying strange mesons, the number of fitted events can also include contributions from non-resonant decays, as well as decays from other states that have masses close to the various daughter masses due to the large mass window cuts. To obtain the number of events for a 

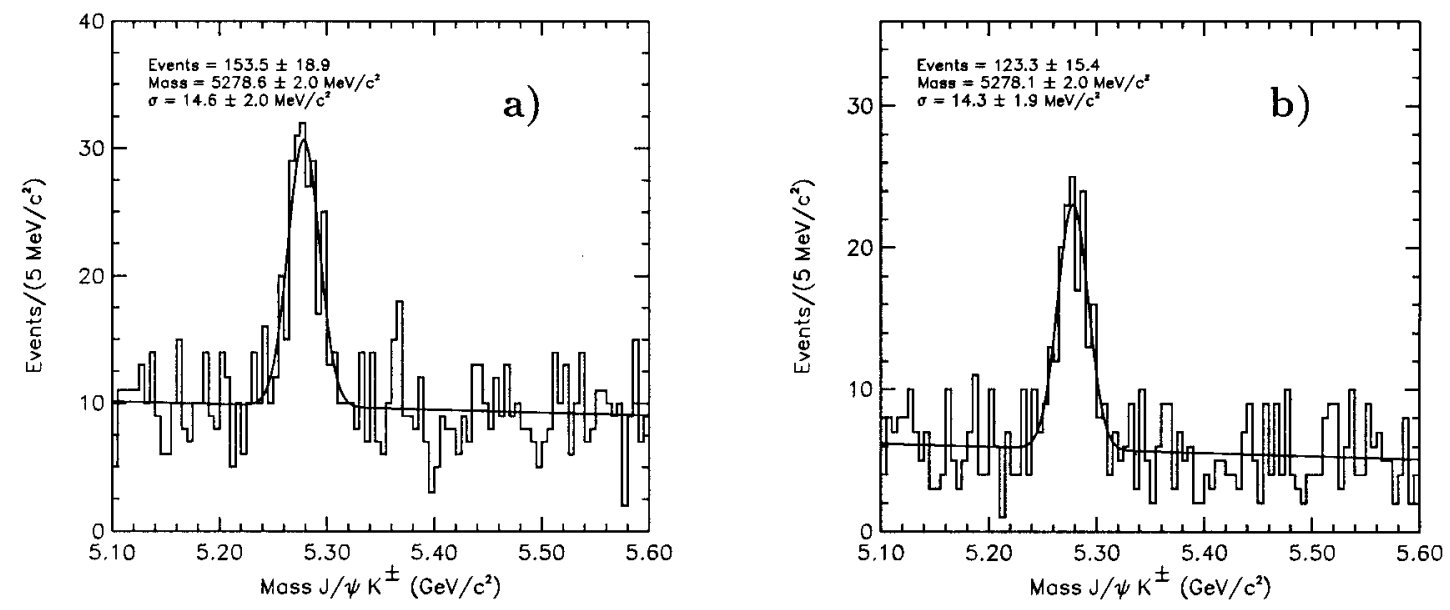

Figure 3.23: Invariant mass distribution for $B^{+} \rightarrow J / \psi K^{+}$candidate events. The momentum cuts on the strange and bottom mesons were 1.5 and $8.0 \mathrm{GeV} / c$ for (a) and 2.0 and $8.0 \mathrm{GeV} / \mathrm{c}$ for (b). The spectra were fitted with a Gaussian distribution and a linear background term.
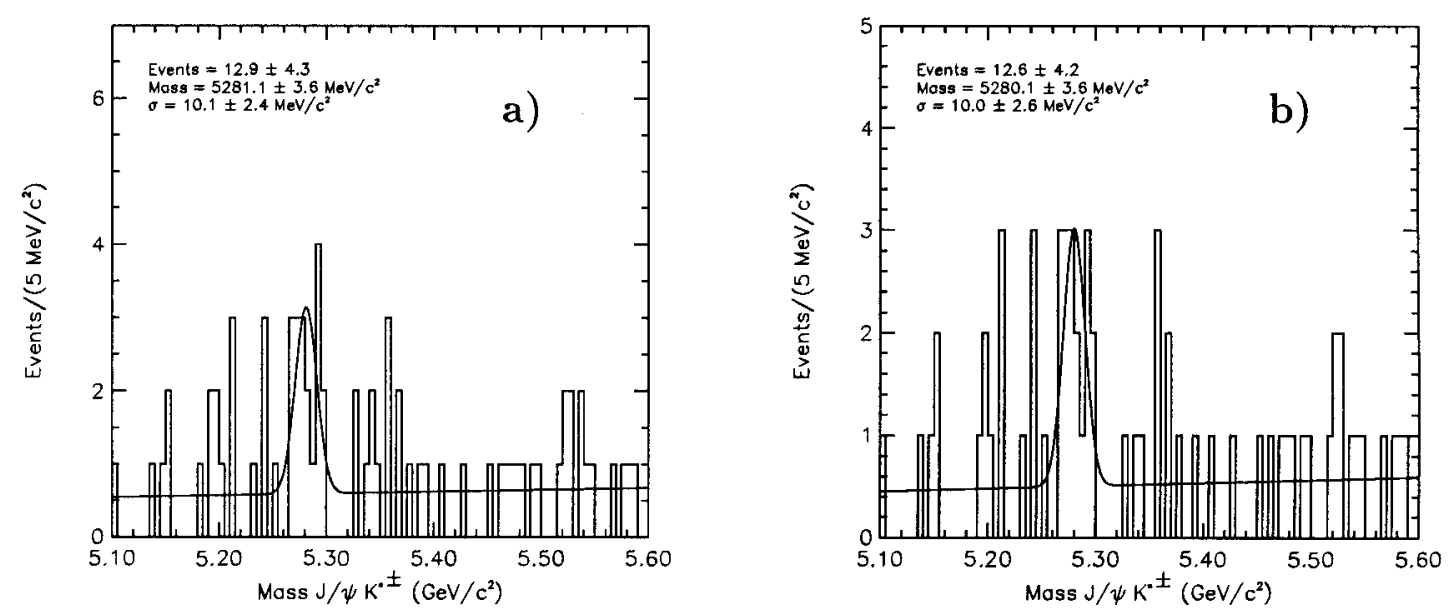

Figure 3.24: Invariant mass distribution for $B^{+} \rightarrow J / \psi K^{*}(892)^{+}$candidate events. The momentum cuts on the strange and bottom mesons were 2.0 and $6.0 \mathrm{GeV} / c$ for (a) and 2.0 and $8.0 \mathrm{GeV} / c$ for (b). The spectra were fitted with a Gaussian distribution and a linear background term. 

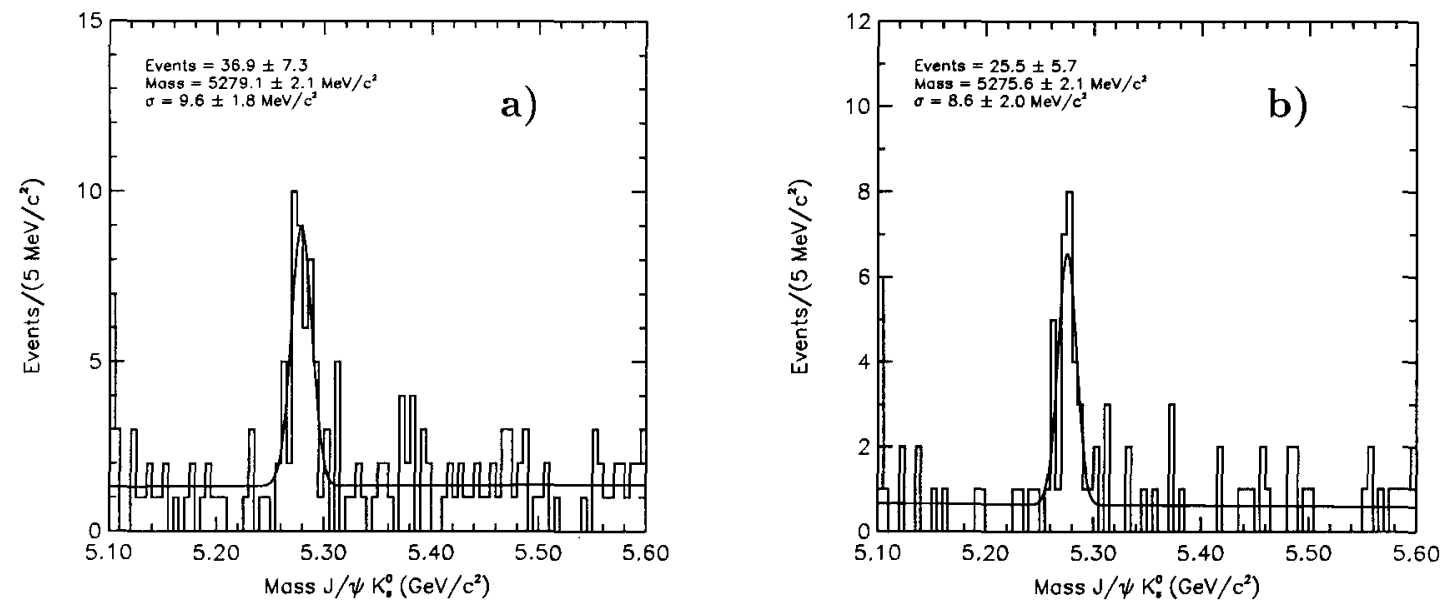

Figure 3.25: Invariant mass distribution for $B^{0} \rightarrow J / \psi K_{s}^{0}$ candidate events. The momentum cuts on the strange and bottom meson were 1.5 and $6.0 \mathrm{GeV} / c$ for (a) and 2.0 and $8.0 \mathrm{GeV} / \mathrm{c}$ for $(\mathrm{b})$. The spectra were fitted with a Gaussian distribution and a linear background term.

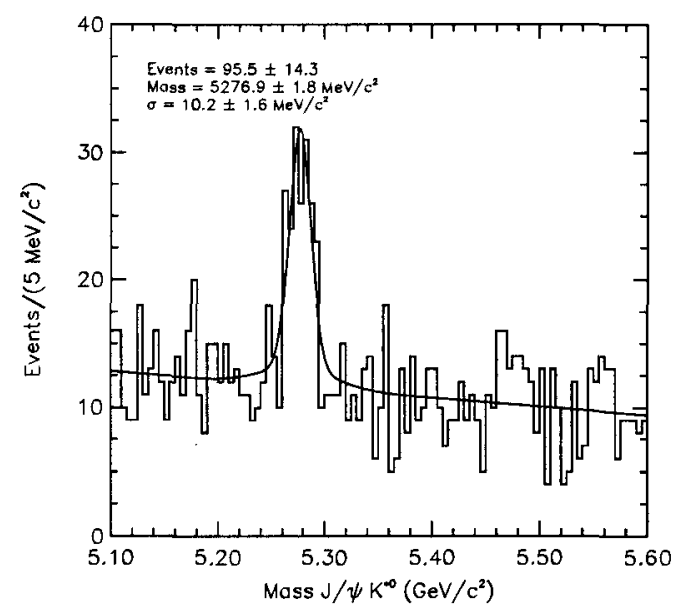

Figure 3.26: Invariant mass distribution for $B^{0} \rightarrow J / \psi K^{*}(892)^{0}$ candidate events. The momentum cuts on the strange and bottom meson were 2.0 and $8.0 \mathrm{GeV} / c$, respectively. The spectrum was fitted with a double Gaussian distribution and a linear background term. The second Gaussian distribution was added to include the cases where the kaon and pion candidates are misassigned. The relative size and width of the two Gaussians distributions was determined by a Monte Carlo calculation. 

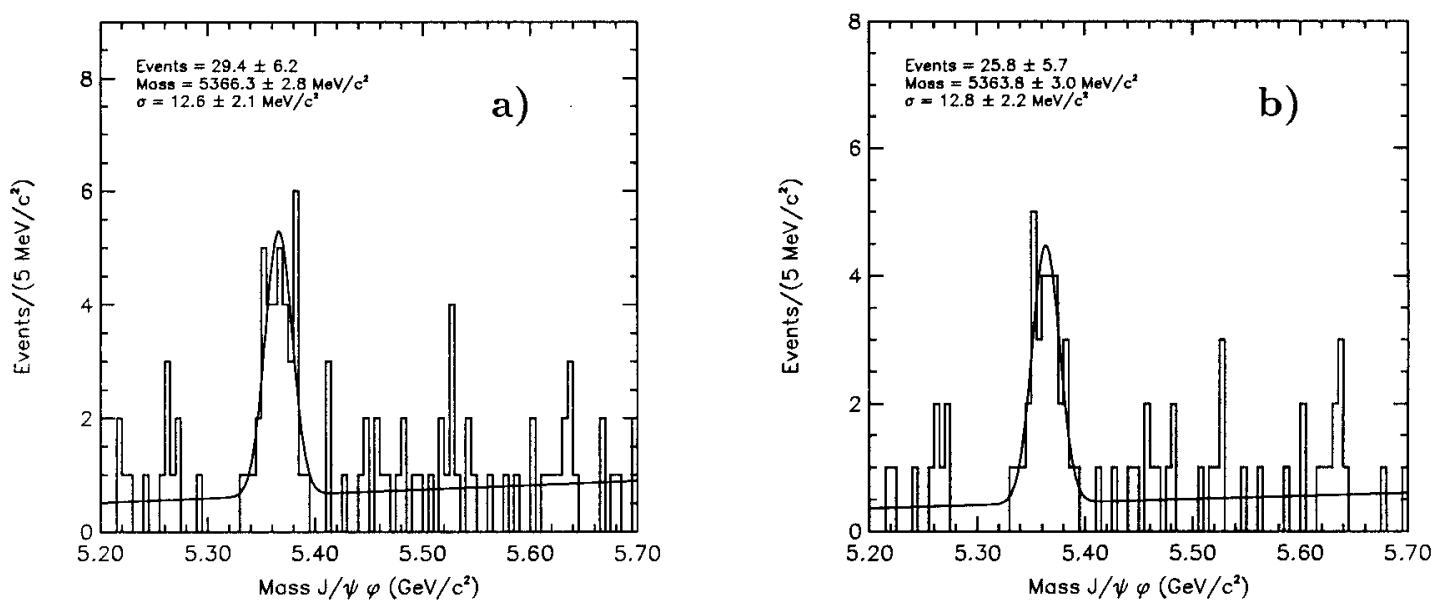

Figure 3.27: Invariant mass distribution for $B_{s}^{0} \rightarrow J / \psi \phi(1020)$ candidate events. The momentum cuts on the strange and bottom meson were 2.0 and $6.0 \mathrm{GeV} / c$ for (a) and 2.0 and $8.0 \mathrm{GeV} / c$ for (b). The spectra were fitted with a Gaussian distribution and a linear background term.

specific resonance decay, the strange daughter invariant mass spectra were examined.

The strange daughter meson invariant mass spectra were obtained by selecting bottom mesons candidates that were in the bottom meson invariant mass signal region. In addition, the invariant mass requirements on the strange mesons were removed. The signal regions for the various decays are listed in Table 3.6. Since the natural widths for the three strong decays are much larger than the detector resolutions, the number of observed decays were then obtained by fitting the respective spectrums to a Breit-Wigner line shape convoluted with a Gaussian distribution. The shapes of the background distributions were taken to be similar to that of the distribution of events taken from the sideband regions of the bottom invariant mass distributions. The strange daughter meson invariant mass spectra are shown in Figures 3.28 to 3.30 and 3.31 to 3.33 and the sideband regions for the various decays are also listed in Table 3.6.

One can also obtain the number of non-resonant decays by subtracting the fitted number of decays from the strange meson invariant mass distributions from the number of events taken from the bottom invariant mass distributions corrected for the efficiency of the mass cuts applied to the strange daughters. Since the resonant and non-resonant decays involve the same particles in the final state, most of the effi- 


\begin{tabular}{|l|c|c|c|}
\hline \hline Decay & $J / \psi K_{s}^{0} \pi^{+}$ & $J / \psi K^{+} \pi^{-}$ & $J / \psi K^{+} K^{-}$ \\
\hline \hline Signal Region $\left(\mathrm{GeV} / c^{2}\right)$ & $5.235-5.325$ & $5.235-5.325$ & $5.320-5.410$ \\
\hline Lower Sideband Region $\left(\mathrm{GeV} / c^{2}\right)$ & $5.000-5.220$ & $5.000-5.180$ & $5.100-5.305$ \\
\hline Higher Sideband Region $\left(\mathrm{GeV} / c^{2}\right)$ & $5.340-5.600$ & $5.380-5.600$ & $5.425-5.700$ \\
\hline \hline
\end{tabular}

Table 3.6: Definition of the signal and sideband regions for bottom decays with strange mesons that strongly decay.

ciencies other than for the mass window cuts should cancel out. For cases where the mass uncertainties are small compared to the natural widths of the strange mesons, the efficiencies for the mass window cut on the strange mesons can be obtained by taking a ratio of the areas parameterized by a Breit-Wigner distribution within the mass window range over the area given by the allowed mass range. These efficiencies are calculated with the use of Equation 3.3.

$\epsilon=\frac{\tan ^{-1}(\Delta M / \Gamma)}{2}\left[\frac{1}{\tan ^{-1}\left(2\left(M_{p}-M_{d 1}-M_{d 2}\right) / \Gamma\right)}+\frac{1}{\tan ^{-1}\left(2\left(M_{B}-M_{J / \psi}-M_{p}\right) / \Gamma\right)}\right]$

In Equation 3.3, $\Delta M$ is the mass window, $M_{p}$ is the mass of the parent particle, $M_{J / \psi}$ is the world average $J / \psi$ mass, $M_{d 1}$ is the mass of the first daughter particle, $M_{d 2}$ is the mass of the second daughter particle and $\Gamma$ is the natural width of the parent particle.

For the case of the $\phi(1020)$ meson, the natural width is small and so the observed width has a significant contribution from the detector resolution. Instead of using Equation 3.3, the efficiency for the $\phi(1020)$ mass window cut can be taken from the inclusive invariant mass resonance. The same can also be done for the $K^{*}(892)^{+}$ resonance. A comparison of the efficiencies obtained from Equation 3.3, Figures 3.10 and 3.11 and a Monte Carlo calculation is shown in Table 3.7.

Using the mass window cut efficiency from the inclusive data for the $\phi(1020)$ meson and the Monte Carlo calculation for the $K^{*}(892)$ mesons, the number of nonresonant $J / \psi K K$ and $J / \psi K \pi$ events can be calculated. It should be noted that the geometric acceptances also depend on the helicity distributions. For simplicity, the helicity distributions for the non-resonant decays are taken to be the same as that for the resonant bottom decays. 


\begin{tabular}{|l|c|c|c|}
\hline \hline Method & $\phi(1020)$ & $K^{*}(892)^{+}$ & $K^{*}(892)^{0}$ \\
\hline \hline Perfect resolution & $88.1 \%$ & $84.0 \%$ & $83.7 \%$ \\
\hline Data & $84.3 \%$ & $85.1 \%$ & \\
\hline MC & $85.5 \%$ & $80.6 \%$ & $80.4 \%$ \\
\hline \hline
\end{tabular}

Table 3.7: Efficiencies for the mass window cuts.
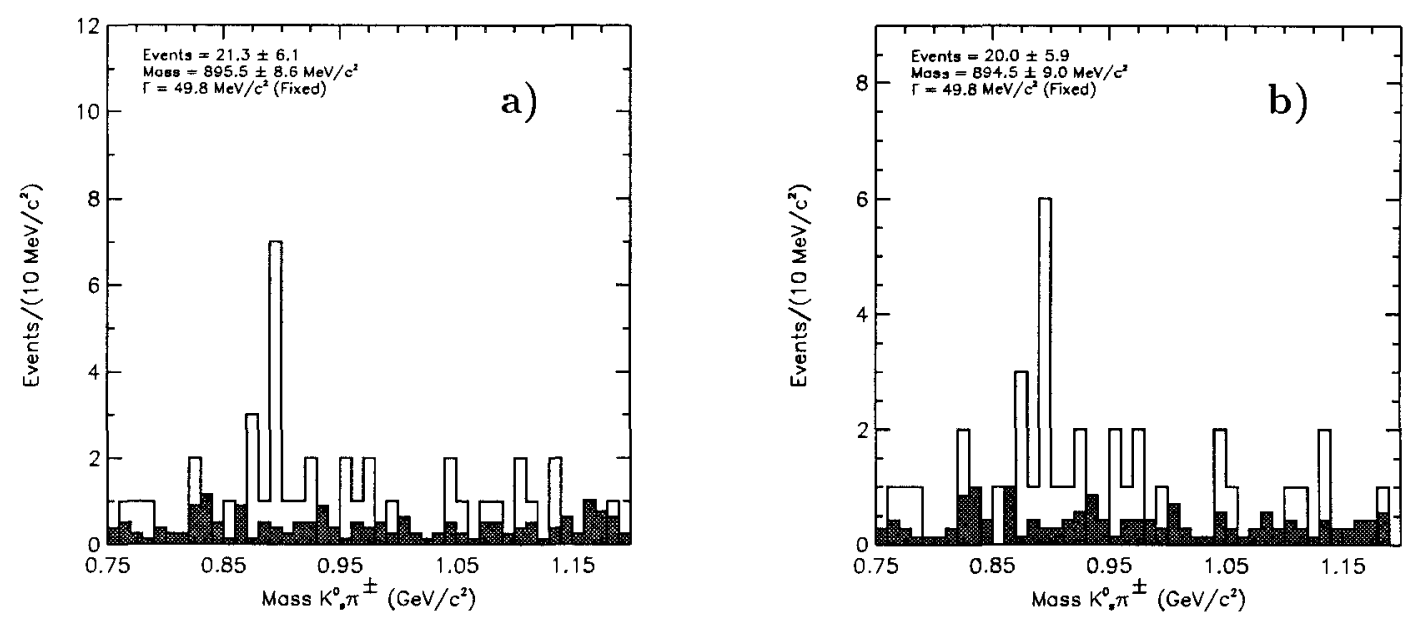

Figure 3.28: Invariant mass distribution for $K^{*}(892)^{+}$candidate events taken from the $B^{+}$mass region. The momentum cuts on the strange and bottom meson were 2.0 and $6.0 \mathrm{GeV} / c$ for (a) and 2.0 and $8.0 \mathrm{GeV} / c$ for (b). The spectra were fitted with a BreitWigner distribution convoluted with a Gaussian distribution. The shaded region represents the non- $B$ background and is normalized to the number of background events under the $B$ resonance. 


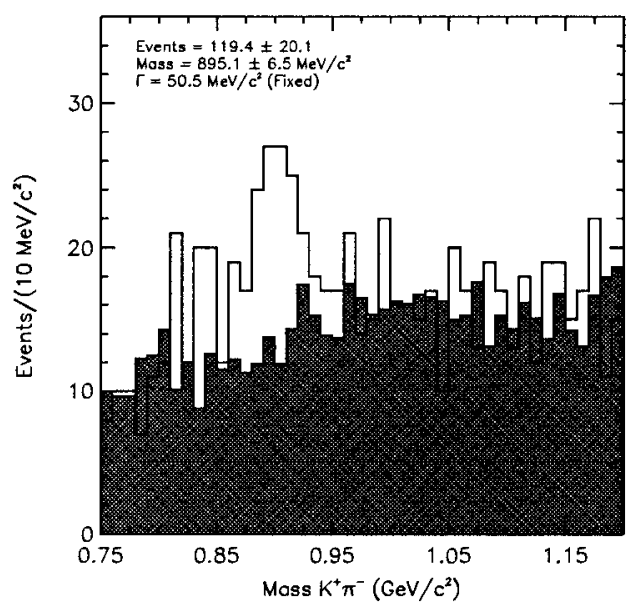

Figure 3.29: Invariant mass distribution for $K^{*}(892)^{0}$ candidate events taken from the $B^{0}$ mass region. The momentum cuts on the strange and bottom meson were 2.0 and 8.0, respectively. The spectrum was fitted with a Breit-Wigner distribution convoluted with a Gaussian distribution. The shaded region represents the background. The shaded region represents the non- $B$ background and is normalized to the number of background events under the $B$ resonance and $K \pi$ flipped events from Monte Carlo. 

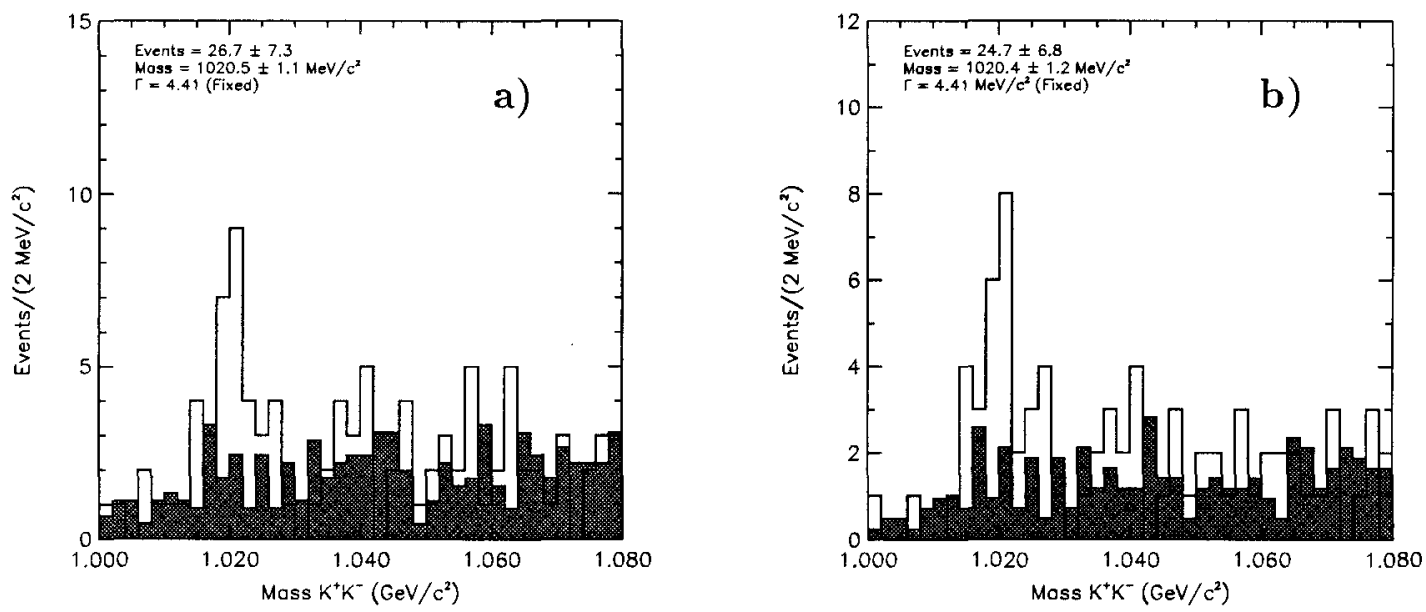

Figure 3.30: Invariant mass distribution for $\phi(1020)$ candidate events taken from the $B_{s}^{0}$ mass region. The momentum cuts on the strange and bottom meson were 2.0 and $6.0 \mathrm{GeV} / c$ for (a) and 2.0 and $8.0 \mathrm{GeV} / c$ for $(\mathrm{b})$. The spectra were fitted with a BreitWigner distribution convoluted with a Gaussian distribution. The shaded region represents the non- $B$ background and is normalized to the number of background events under the $B$ resonance.
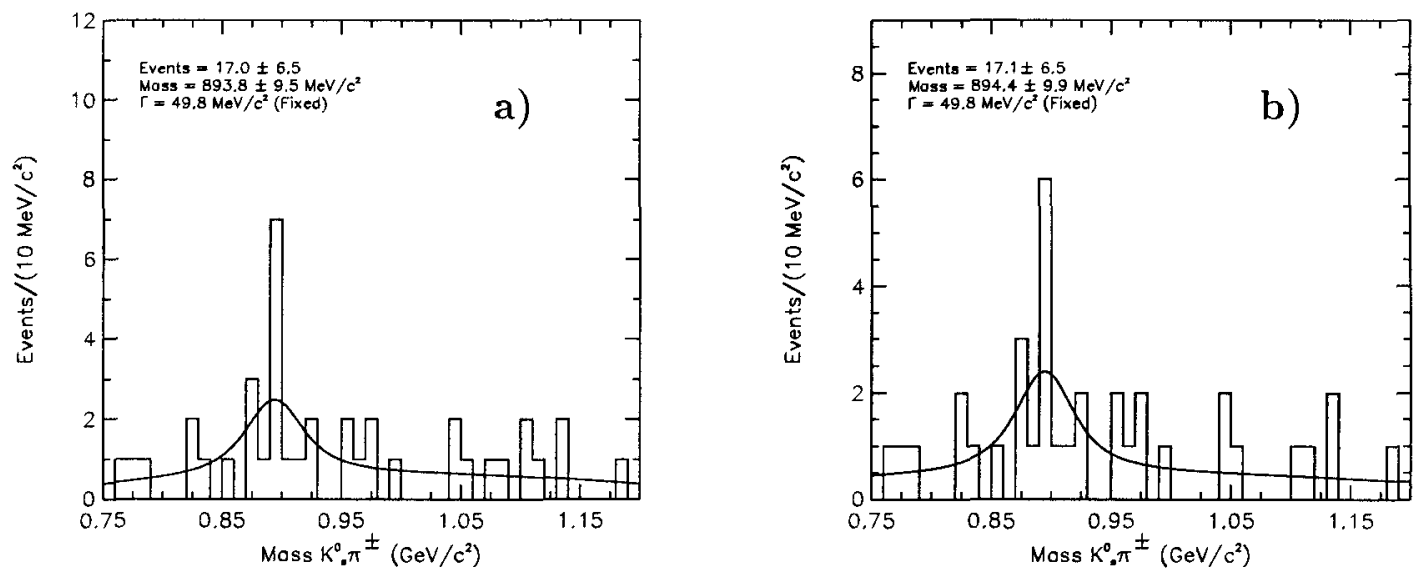

Figure 3.31: Invariant mass distribution for $K^{*}(892)^{+}$candidate events taken from the $B^{+}$mass region. The momentum cuts on the strange and bottom meson were 2.0 and $6.0 \mathrm{GeV} / c$ for $(\mathrm{a})$ and 2.0 and $8.0 \mathrm{GeV} / c$ for $(\mathrm{b})$. The spectra were fitted with a Breit-Wigner distribution convoluted with a Gaussian distribution. The background was taken to be a second-order polynominal. 


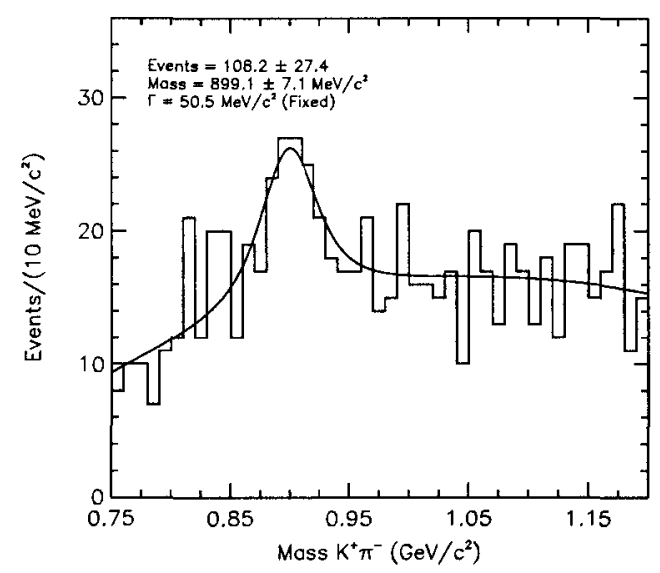

Figure 3.32: Invariant mass distribution for $K^{*}(892)^{0}$ candidate events taken from the $B^{0}$ mass region. The momentum cuts on the strange and bottom meson were 2.0 and $8.0 \mathrm{GeV} / c$, respectively. The spectrum was fitted with a Breit-Wigner distribution convoluted with a Gaussian distribution.
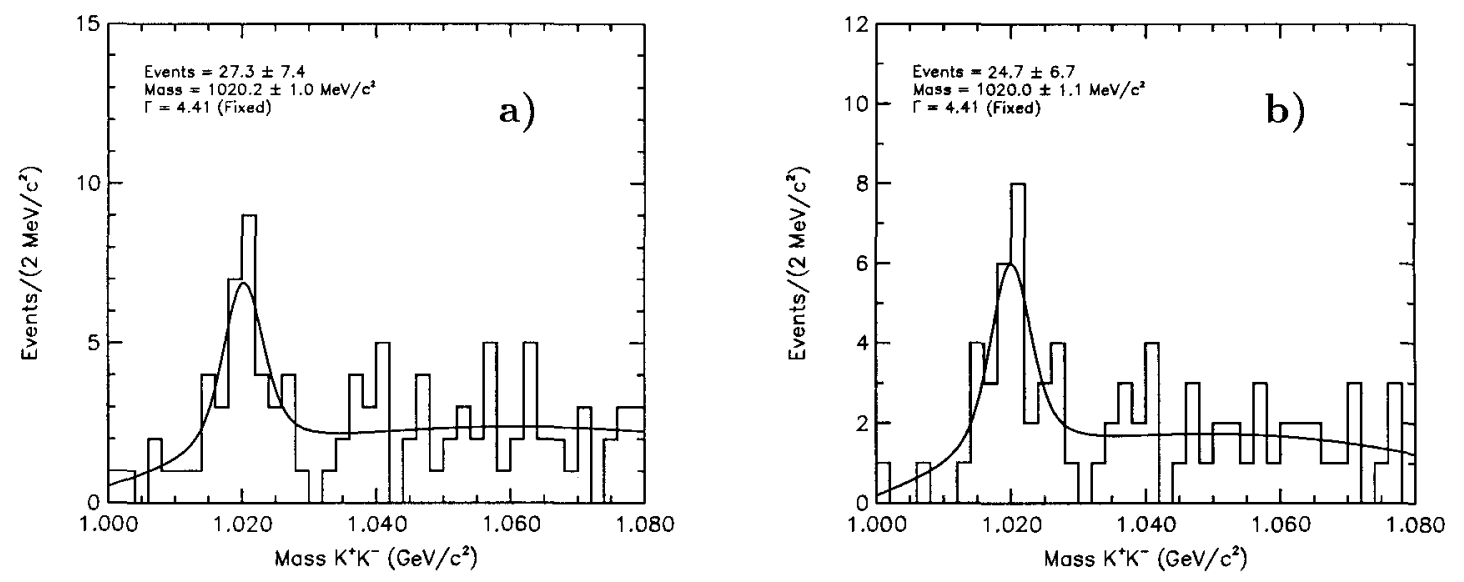

Figure 3.33: Invariant mass distribution for $\phi(1020)$ candidate events taken from the $B_{s}^{0}$ mass region. The momentum cuts on the strange and bottom meson were 2.0 and $6.0 \mathrm{GeV} / c$ for (a) and 2.0 and $8.0 \mathrm{GeV} / c$ for (b). The spectra were fitted with a Breit-Wigner distribution convoluted with a Gaussian distribution. The background was taken to be a second-order polynominal. 


\begin{tabular}{|l|c|}
\hline \hline Decay & Number of Events \\
\hline \hline$B^{+} \rightarrow J / \psi K^{+}$ & $153.5 \pm 18.9$ \\
\hline$B^{+} \rightarrow J / \psi K^{*}(892)^{+}$ & $21.3 \pm 6.1$ \\
\hline$B^{+} \rightarrow J / \psi K_{s}^{0} \pi^{+}$ & $12.9 \pm 4.3$ \\
\hline$B^{+}(N R) \rightarrow J / \psi K_{s}^{0} \pi^{+}$ & $-4.3 \pm 6.5$ \\
\hline$B^{0} \rightarrow J / \psi K_{s}^{0}$ & $36.9 \pm 7.3$ \\
\hline$B^{0} \rightarrow J / \psi K^{*}(892)^{0}$ & $119.4 \pm 20.1$ \\
\hline$B^{0} \rightarrow J / \psi K^{+} \pi^{-}$ & $95.5 \pm 14.3$ \\
\hline$B^{0}(N R) \rightarrow J / \psi K^{+} \pi^{-}$ & $-0.5 \pm 21.6$ \\
\hline$B_{s}^{0} \rightarrow J / \psi \phi(1020)$ & $26.7 \pm 7.3$ \\
\hline$B_{s}^{0} \rightarrow J / \psi K^{+} K^{-}$ & $29.4 \pm 6.2$ \\
\hline$B_{s}^{0}(N R) \rightarrow J / \psi K^{+} K^{-}$ & $6.6 \pm 8.8$ \\
\hline \hline
\end{tabular}

Table 3.8: Number of reconstructed $B$ meson including non-resonant decays using different momentum cuts. The label NR represents the projected number of nonresonant decays.

The number of reconstructed bottom mesons decays including the non-resonant decays are summarized in Tables 3.8 and 3.9. By examining Figures 3.28 to 3.30 and the calculated values in Tables 3.8 and 3.9 , one can conclude that there are no significant non-resonant contributions above the non- $B$ backgrounds.

Because the background subtracted invariant mass distributions for the daughter strange mesons is least biased by potential contributions from small amounts of nonresonant production, they were the distributions that are used to obtain the number of bottom decays. As a cross-check on the fitting procedure, the number of signal events was also obtained by fitting the invariant mass of the strange mesons to a Breit-Wigner convoluted with a Gaussian distribution and the background described by a second-order polynomial function. The resulting fits are shown in Figures 3.31 to 3.33. The number of fitted events were found to be consistent. 


\begin{tabular}{|l|c|}
\hline \hline Decay & Number of Events \\
\hline \hline$B^{+} \rightarrow J / \psi K^{+}$ & $123.3 \pm 15.4$ \\
\hline$B^{+} \rightarrow J / \psi K^{*}(892)^{+}$ & $20.0 \pm 5.9$ \\
\hline$B^{+} \rightarrow J / \psi K_{s}^{0} \pi^{+}$ & $12.6 \pm 4.2$ \\
\hline$B^{+}(N R) \rightarrow J / \psi K_{s}^{0} \pi^{+}$ & $-3.5 \pm 6.3$ \\
\hline$B^{0} \rightarrow J / \psi K_{s}^{0}$ & $25.5 \pm 5.7$ \\
\hline$B^{0} \rightarrow J / \psi K^{*}(892)^{0}$ & $119.4 \pm 20.1$ \\
\hline$B^{0} \rightarrow J / \psi K^{+} \pi^{-}$ & $95.5 \pm 14.3$ \\
\hline$B^{0}(N R) \rightarrow J / \psi K^{+} \pi^{-}$ & $-0.5 \pm 21.6$ \\
\hline$B_{s}^{0} \rightarrow J / \psi \phi(1020)$ & $24.7 \pm 6.8$ \\
\hline$B_{s}^{0} \rightarrow J / \psi K^{+} K^{-}$ & $25.8 \pm 5.7$ \\
\hline$B_{s}^{0}(N R) \rightarrow J / \psi K^{+} K^{-}$ & $4.7 \pm 8.1$ \\
\hline \hline
\end{tabular}

Table 3.9: Number of reconstructed $B$ meson including non-resonant decays using the similar momentum cuts. The label NR represents the projected number of nonresonant decays. 


\section{Chapter 4}

\section{Calculation of Efficiencies}

Since all five bottom decays have similar topologies, the efficiencies as well as the ratios of efficiencies should be similar. Since the $J / \psi$ requirements were the same for all of the channels, the ratio of efficiencies of the muon and $J / \psi$ requirements will cancel out.

However, there are a few ratios of efficiencies that will not cancel out in a ratio. The efficiencies that do not cancel out include the kinematic and geometric efficiencies, lifetime cut efficiencies, CTC pattern finding efficiencies and confidence level cut efficiencies. Other efficiencies that do not cancel in the ratios are the mass window cut efficiencies. Of course, these efficiencies are only relevant to the non-resonance bottom type decays. The ratio of the detection efficiencies that are relevant for the bottom decays are described in the following sections.

\subsection{Kinematic and Geometric Efficiencies}

The most important efficiencies used in this analysis are the kinematic and geometrical acceptances. These efficiencies were determined with the use of Monte Carlo calculations. The Monte Carlo calculations were performed in the steps listed below.

1. Generation of underlying events in $\bar{p} p$ collisions.

2. Generation of bottom quarks and hadronization to the various bottom mesons.

3. Decay of the bottom mesons with the most current decay information available.

4. Simulation of the CDF detector response to the decay fragments. 
5. Reconstruction of the event information.

6. Simulation of the level 1 and level $2 \mathrm{CDF}$ dimuon trigger.

7. Application of the data selection requirements.

Underlying events in $\bar{p} p$ collisions were produced with the ISAJET generator [56]. The inclusion of the underlying events is important for several reasons. The efficiency to reconstruct charged tracks is dependent on the number of CTC wire hits. This in turn is dependent on the charged track multiplicities and instantaneous luminosity. Thus, one must correctly model the underlying event to calculate efficiencies that depend on the track multiplicities in the tracking chambers. It should be noted that the ISAJET generator was modified so as to include the most recent particle masses, natural widths and lifetimes taken from the world averages as calculated by the Particle Data Group [7].

In addition to the track reconstruction efficiency, the calculation of the primary $\bar{p} p$ $z$ vertex position is also dependent on the number of charged tracks available. With only three to five tracks in the various bottom meson decays, the determination of the primary $z$ vertex position is poor and the $B$ vertex is treated as the primary vertex which is incorrect. So once again, it is important to model the number of charged tracks correctly.

The $B$ meson $c \tau$ cut efficiency is also dependent on the number of SVX-CTC tracks found, which depends on the number of recorded hits in the SVX detector (occupancy). The SVX occupancy depends on the number of charged particles and so is dependent on the instantaneous luminosity and the number of charged tracks present in the event.

In order to produce the correct charge multiplicity distribution for the $J / \psi$ events, the ISAJET generator was used to produce dijet events to model the underlying event. The minimum transverse momentum of the partons that produced the jets was set to $15 \mathrm{GeV} / c$ so that the generated charged track distribution was similar to that observed in the data.

With the inclusion of particles from the underlying event, the charge multiplicity distribution from the data and from the Monte Carlo calculation are roughly the same. The two charge multiplicity distributions are shown in Figure 4.1. The respective total momentum and transverse momentum distributions are shown in Figures 4.2 


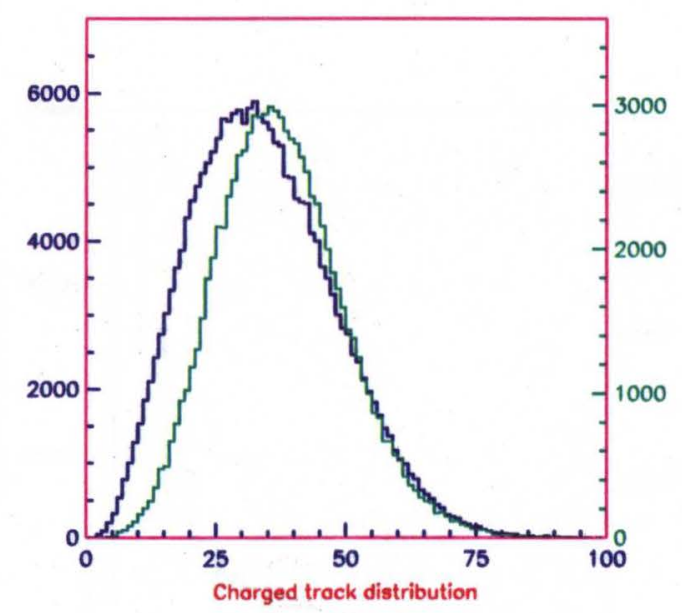

Figure 4.1: Track multiplicity distribution for data (blue histogram) and Monte Carlo events (green histogram).

and 4.3. There is reasonable agreement between the distributions taken from data and the Monte Carlo calculation.

The generation of bottom quarks and hadronization to the various bottom mesons was done within a certain theoretical framework. The theoretical model describing the $b$ quark production properties involves a next-to-leading order QCD calculation [11, $12,16]$, with the renormalization scale $\mu_{0}=\sqrt{m_{b}^{2}+P_{T}^{2}}$, where the $b$ quark mass, $m_{b}$, is set to $4.75 \mathrm{GeV} / c^{2}$. The $b$ quarks were produced with a transverse momentum greater than $5.0 \mathrm{GeV} / c$. The model uses the $\mathrm{MRSD}_{0}$ parton distribution functions [14] and the Peterson parameterization $[17,18]$ for the $b$ quark fragmentation functions. The Peterson fragmentation parameter $(\epsilon)$ was set to 0.006 for $b$ decays.

The CLEO Monte Carlo program [57] was used to decay the bottom mesons with the most recent particle masses, natural widths and lifetimes taken from the world averages as calculated by the PDG [7]. The CLEO Monte Carlo program was also modified to include the most recent helicity distributions for the relevant decays [58]. The amount of longitudinal polarization used for the various decays that involved two vector daughter particles from the parent bottom mesons are listed in Table 4.1.

After the $B$ mesons were generated, the events were processed with a complete simulation of the detector response. The primary vertex position of the generated events in both the transverse and longitudinal plane was distributed approximately in 


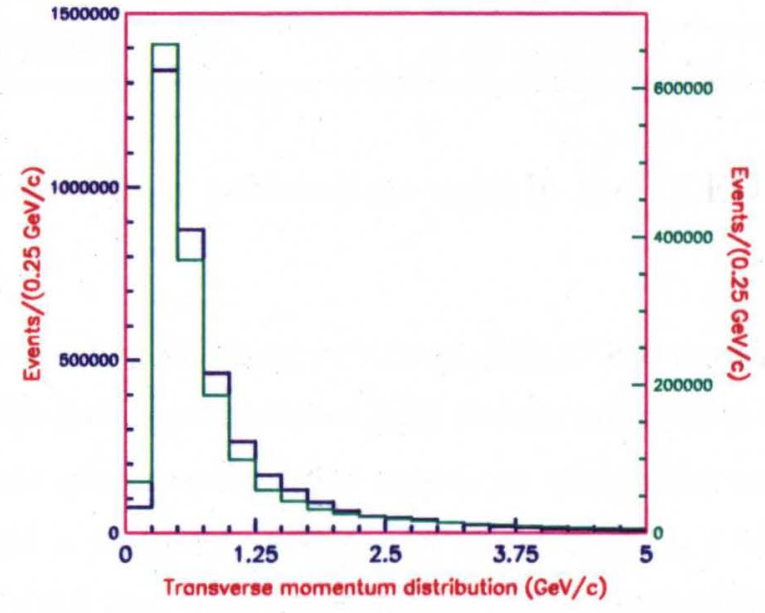

Figure 4.2: Transverse momentum distribution of tracks in data (blue histogram) and Monte Carlo events (green histogram).

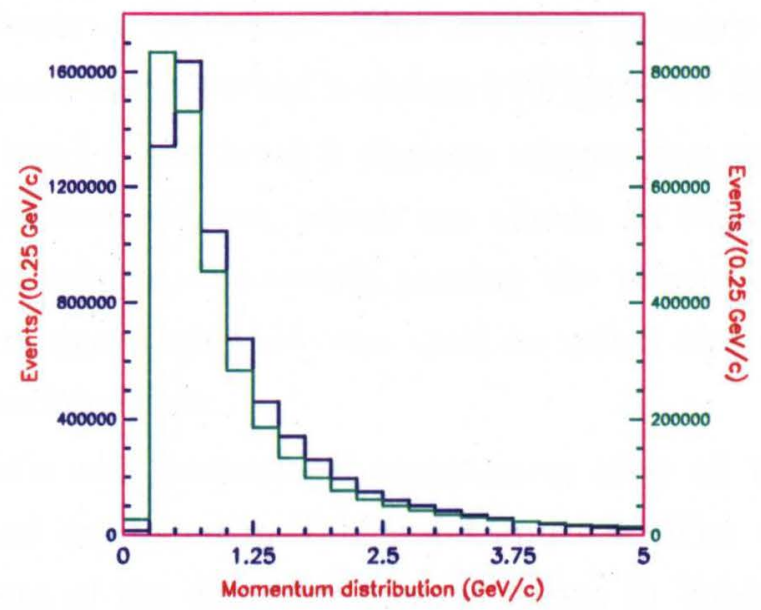

Figure 4.3: Total momentum distribution of tracks in data (blue histogram) and Monte Carlo events (green histogram). 


\begin{tabular}{|l|c|}
\hline \hline Decay & Amount of Longitudinal Polarization \\
\hline \hline$B^{+} \rightarrow J / \psi K^{*}(892)^{+}$ & $0.75 \pm 0.10$ \\
\hline$B^{0} \rightarrow J / \psi K^{*}(892)^{0}$ & $0.75 \pm 0.10$ \\
\hline$B_{s}^{0} \rightarrow J / \psi \phi(1020)$ & $0.50 \pm 0.25$ \\
\hline \hline
\end{tabular}

Table 4.1: Amount of longitudinal polarization used in the CLEO Monte Carlo calculations.

the same manner as the observed primary vertex position. For example, the primary $z$ vertex distributions for the data and Monte Carlo events are shown in Figure 4.4. The spike at $z=0$ for the data plot results from events in which the reconstruction code could not successfully find a primary vertex. For those events, $z$ was set to zero and the uncertainty was set to $0.3 \mathrm{~cm}$. The distribution of the uncertainty on the primary $z$ vertex for the both the data and Monte Carlo events are shown in Figure 4.5. The approximate agreement is adequate since the reconstruction efficiencies are relatively insensitive to the assigned primary vertex uncertainty.

The transverse beam profile is shown in Figure 4.6 (a). The Monte Carlo calculated $(x, y)$ values were approximated by two correlated Gaussian distributions rotated by 45 degrees. The standard deviations, means and respective amplitudes of the two Gaussians were approximated to give roughly the same transverse beam profile as observed in Tevatron collisions. The resulting primary transverse vertex distribution from the Monte Carlo events is shown in Figure 4.6 (b).

A simulation of the level 1 and level 2 dimuon trigger was performed by using the measured trigger efficiency curves, which are shown in Figure 3.9. After the simulation of the dimuon trigger, the events passing the trigger requirements were processed with the same algorithm that was used to select the candidate bottom meson decays from the collider data.

The resulting kinematic and geometrical acceptances after all the event selection requirements were applied are listed in Tables 4.3 and 4.4. The values include the various branching fractions of the daughter mesons shown in Table 4.2. The respective Monte Carlo statistical uncertainties on the efficiencies given in Table 4.4 were $1.3 \%, 2.0 \%, 2.1 \%, 1.3 \%$ and $1.1 \%$. Although more Monte Carlo statistics would have resulted in smaller statistical uncertainties, there is little benefit in reducing this uncertainty. It is unreasonable to assume the accuracy of the simulation is better than 

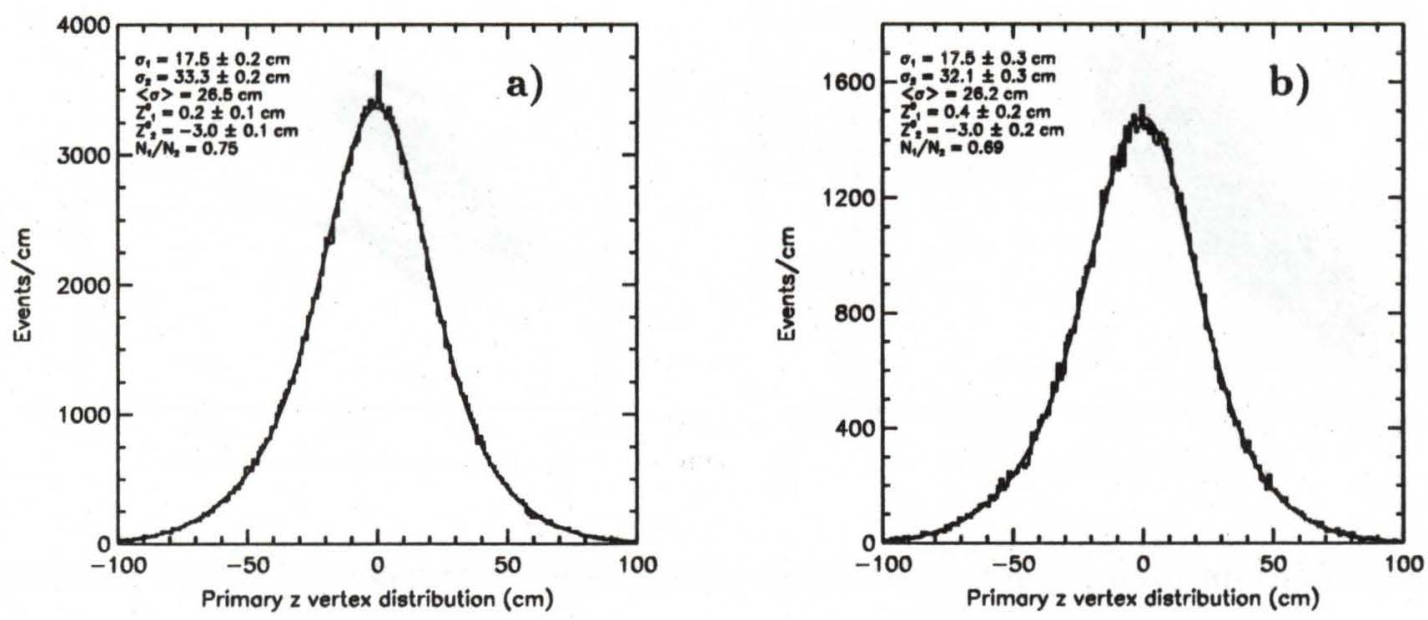

Figure 4.4: Primary $z$ vertex distribution for (a) data and (b) Monte Carlo events.

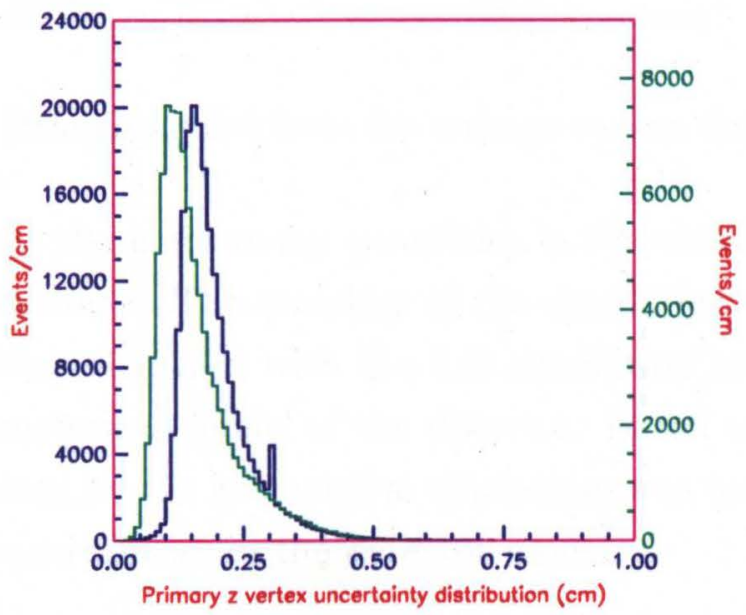

Figure 4.5: Primary $z$ vertex uncertainty distribution for data (blue histogram) and Monte Carlo events (green histogram). 

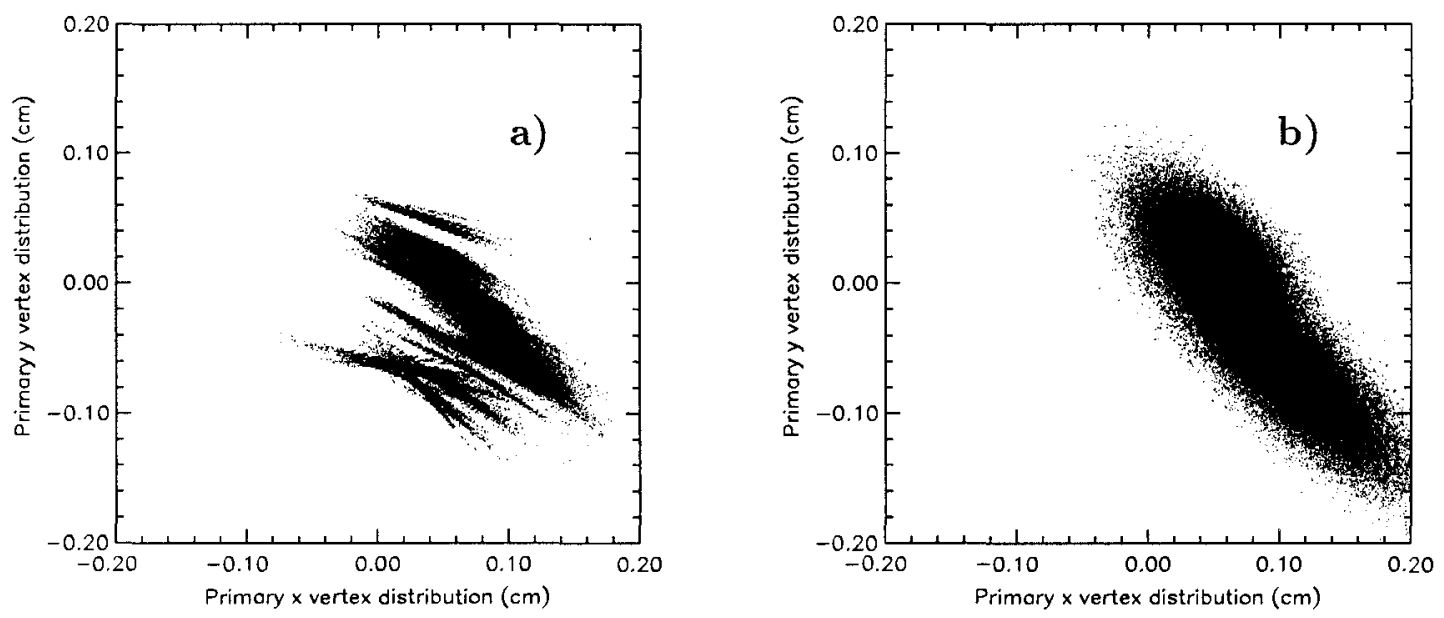

Figure 4.6: Primary vertex transverse profile distribution for (a) data and (b) Monte Carlo events.

\begin{tabular}{|l|c|}
\hline \hline Decay & Branching Fraction \\
\hline \hline$\phi(1020) \rightarrow K^{+} K^{-}$ & $(49.1 \pm 0.9) \%$ \\
\hline$K_{s}^{0} \rightarrow \pi^{+} \pi^{-}$ & $(68.61 \pm 0.28) \%$ \\
\hline$K^{0} \rightarrow K_{s}^{0}$ & $1 / 2$ \\
\hline$K^{*}(892)^{+} \rightarrow K^{0} \pi^{+}$ & $2 / 3$ \\
\hline$K^{*}(892)^{0} \rightarrow K^{+} \pi^{-}$ & $2 / 3$ \\
\hline \hline
\end{tabular}

Table 4.2: Branching fractions for strange meson decays.

of order one percent, since there are many quantities in the simulation that are approximated by reasonable values. The accuracy of the simulation was determined by comparing the acceptances calculated with the full simulation and the acceptances determined using a parameterized model of the detector. Based on this comparison, a $5 \%$ systematic uncertainty on the geometrical efficiencies was assigned to take into account any remaining uncertainties in the detector model.

\subsection{Proper Decay Length Cut Efficiencies}

Since the various $B$ mesons have similar lifetimes, the ratios of efficiencies for the bottom meson $c \tau$ cut should be approximately 1.0. Nevertheless, the actual efficien- 


\begin{tabular}{|l|c|}
\hline \hline Decay & Kinematic and Geometrical Acceptances \\
\hline \hline$B^{+} \rightarrow J / \psi K^{+}$ & $0.663 \%$ \\
\hline$B^{+} \rightarrow J / \psi K^{*}(892)^{+}$ & $0.0619 \%$ \\
\hline$B^{0} \rightarrow J / \psi K^{0}$ & $0.177 \%$ \\
\hline$B^{0} \rightarrow J / \psi K^{*}(892)^{0}$ & $0.334 \%$ \\
\hline$B_{s}^{0} \rightarrow J / \psi \phi(1020)$ & $0.313 \%$ \\
\hline
\end{tabular}

Table 4.3: Kinematic and geometrical acceptances for the decays with different momentum requirements on the bottom and strange mesons.

\begin{tabular}{|l|c|}
\hline \hline Decay & Kinematic and Geometrical Acceptances \\
\hline \hline$B^{+} \rightarrow J / \psi K^{+}$ & $0.584 \%$ \\
\hline$B^{+} \rightarrow J / \psi K^{*}(892)^{+}$ & $0.0588 \%$ \\
\hline$B^{0} \rightarrow J / \psi K^{0}$ & $0.136 \%$ \\
\hline$B^{0} \rightarrow J / \psi K^{*}(892)^{0}$ & $0.334 \%$ \\
\hline$B_{s}^{0} \rightarrow J / \psi \phi(1020)$ & $0.278 \%$ \\
\hline
\end{tabular}

Table 4.4: Kinematic and geometrical acceptances for the decays with similar momentum requirements on the bottom and strange mesons. 


\begin{tabular}{|l|c|c|c|c|c|}
\hline \hline Decay/\# of Tracks with SVX Hits & 0 & 1 & 2 & 3 & 4 \\
\hline \hline$B^{+} \rightarrow J / \psi K^{+}$ & 440 & 114 & 72.5 & 58.4 & \\
\hline$B^{+} \rightarrow J / \psi K^{*}(892)^{+}$ & 487 & 341 & 83.1 & 61.5 & \\
\hline$B^{0} \rightarrow J / \psi K_{s}^{0}$ & 462 & 246 & 67.4 & & \\
\hline$B^{0} \rightarrow J / \psi K^{*}(892)^{0}$ & 490 & 159 & 79.4 & 66.6 & 56.8 \\
\hline$B_{s}^{0} \rightarrow J / \psi \phi(1020)$ & 472 & 126 & 82.0 & 65.7 & 57.6 \\
\hline \hline
\end{tabular}

Table 4.5: $c \tau$ standard deviations in $\mu \mathrm{m}$ of sideband events with various number of tracks with SVX hits.

\begin{tabular}{|l|c|c|c|}
\hline \hline Decay & Central & Low & High \\
\hline \hline$B^{+} \rightarrow J / \psi K^{+}$ & $90.0 \%$ & $89.5 \%$ & $90.5 \%$ \\
\hline$B^{+} \rightarrow J / \psi K^{*}(892)^{+}$ & $88.0 \%$ & $87.4 \%$ & $88.5 \%$ \\
\hline$B^{0} \rightarrow J / \psi K_{s}^{0}$ & $87.6 \%$ & $86.9 \%$ & $88.2 \%$ \\
\hline$B^{0} \rightarrow J / \psi K^{*}(892)^{0}$ & $89.3 \%$ & $88.7 \%$ & $89.8 \%$ \\
\hline$B_{s}^{0} \rightarrow J / \psi \phi(1020)$ & $88.4 \%$ & $86.0 \%$ & $90.0 \%$ \\
\hline \hline
\end{tabular}

Table 4.6: $c \tau$ cut efficiency values for the various decays using the central values and one standard deviation of the $c \tau$ values taken from the PDG.

cies can be determined by examining the standard deviations of the proper decay length distributions of background events. Background events were chosen from the sideband invariant mass regions of the bottom meson candidates. The standard deviations of the proper decay lengths $(c \tau)$ for the various decays of interest as a function of the number of tracks that have SVX hits is shown in Table 4.5.

The expected fraction of tracks that have SVX hits in each decay was determined with the use of the Monte Carlo calculation described in Section 4.1. The distribution of the proper decay length for the Monte Carlo events were then smeared with the appropriate values taken from Table 4.5. The resulting efficiencies for the various bottom meson decays are shown in Table 4.6. The values and standard deviations given in Table 4.6 were obtained by using the different transverse momentum requirements on the bottom and strange mesons. The results do not significantly change if the similar transverse momentum requirements on the strange and bottom mesons are used.

The $K_{s}^{0} c \tau$ cut efficiency can be determined using two methods. The first method 


\begin{tabular}{|l|c|}
\hline \hline Method & $K_{s}^{0}$ Lifetime Cut Efficiency \\
\hline \hline$B^{+}$sideband events & $95.9 \%$ \\
\hline$B^{0}$ sideband events & $94.9 \%$ \\
\hline Fig. 3.18 & $96.6 \%$ \\
\hline Fig. 3.19 & $95.8 \%$ \\
\hline \hline
\end{tabular}

Table 4.7: $c \tau$ cut efficiency values for $K_{s}^{0}$ mesons using various methods.

is similar to that which was described above. The efficiency can also be determined from the inclusive plots shown in Figures 3.18 and 3.19. The various lifetime cut efficiencies using the different methods are shown in Table 4.7. The average of the four values is $(95.8 \pm 0.7) \%$ where the uncertainty is the standard deviation of the four methods.

\subsection{CTC Pattern Finding Efficiencies}

The CTC pattern recognition efficiency is defined as the fraction of charged tracks that traverse the CTC chamber in which the track finding algorithm successfully reconstructs the track. This efficiency is strongly correlated with the number of CTC sense wires that register a hit. For this reason it is expected that the efficiency decreases with increasing instantaneous luminosity as the overlap of hits in the CTC layers with larger track multiplicities reduces the number of acceptable hits for a track. This efficiency was determined by embedding simulated hits on the sense wires in dimuon data events that were used for this analysis.

The embedding and reconstruction procedure was broken down into five steps. For the kaon from the $B^{+} \rightarrow J / \psi K^{+}$decay, the first step was to tag the candidate kaons that passed all of the selection requirements. The second step involved recording the five track parameters for each candidate event. Then, simulated hits based on the same five track parameters with the exception of an opposite signed curvature value was embedded into the data events. The fourth step involved rerunning the track reconstruction. The fifth step involved determining which of the embedded tracks were successfully reconstructed. The embedded tracks were considered to be successfully reconstructed if the difference in the track parameters between the original and embedded track over the uncertainties of the track parameters were within three 
standard deviations. The CTC pattern recognition efficiency is then the ratio of the number of found tracks over the number of embedded tracks. The calculated efficiency using this procedure was determined to be $(98.2 \pm 0.7) \%$, where the uncertainty is due to statistics.

The same method was used for the $\phi(1020)$ candidates. The resulting combined $\phi(1020)$ CTC pattern recognition efficiency was determined to be $(94.1 \pm 2.1) \%$. One might argue that for two tracks with the same sign of curvature, this method may lead to an inaccurate estimate because of possible correlations between the two $\phi(1020)$ daughters. This is not expected to be a large effect because the two tracks would typically be separated in $\eta$ and differ in the magnitude of curvature. Empirically, one notes that the ratio of same charge dikaons to opposite charge dikaons events is in reasonable agreement with the predicted value based on the observed charge multiplicity, supporting the assumption of no efficiency correlations between the tracks.

Because the $K_{s}^{0}$ meson is long lived $(c \tau=2.676 \mathrm{~cm})$, the CTC pattern recognition efficiency for the $K_{s}^{0}$ daughter tracks could be very different from the values determined for the mesons mentioned above. Using the same method as described before, hits for each of the pion candidates (one leg at a time) were added with the opposite signed curvature value into data events. This gave a combined efficiency for both tracks of $(88.7 \pm 2.5) \%$.

The CTC pattern finding efficiency was also determined using a second method. For the kaons from the $B_{s}^{0} \rightarrow J / \psi \phi(1020)$ decay, the CTC pattern finding efficiency was determined by embedding hits described by track parameters that were obtained from a Monte Carlo calculation based on the expected topology of the $B_{s}^{0} \rightarrow J / \psi \phi(1020)$ decay. The hits were then embedded in data events that satisfied the selection requirements of any of the other four bottom mesons decays. The CTC pattern finding efficiencies for the $K_{s}^{0}$ and $K^{*}$ mesons were determined with the use of this second procedure. It should be noted that this procedure is reasonable only if the Monte Carlo calculations accurately model the $B$ production and decay kinematics. To test whether this assumption is valid, the ratio of found events with all the tracks exiting through the outer CTC can and with at least one track exiting through the CTC endplate are compared for the Monte Carlo calculation and data. This ratio is used because the pattern recognition efficiency cancels out. The results for the five decays are shown in Table 4.8. As one can see from Table 4.8, the agreement between the Monte Carlo calculation and data is reasonable, validating the use 


\begin{tabular}{|l|c|}
\hline \hline Decay & Relative Ratio of Found Events \\
\hline \hline$B^{+} \rightarrow J / \psi K^{+}$ & $(102 \pm 2) \%$ \\
\hline$B^{+} \rightarrow J / \psi K^{*}(892)^{+}$ & $(100 \pm 6) \%$ \\
\hline$B^{0} \rightarrow J / \psi K_{s}^{0}$ & $(95 \pm 6) \%$ \\
\hline$B^{0} \rightarrow J / \psi K^{*}(892)^{0}$ & $(100 \pm 3) \%$ \\
\hline$B_{s}^{0} \rightarrow J / \psi \phi(1020)$ & $(96 \pm 6) \%$ \\
\hline \hline
\end{tabular}

Table 4.8: Relative ratio of found events with all tracks exiting the outer CTC can and all found events between Monte Carlo and data.

\begin{tabular}{|l|c|}
\hline \hline Particle & Track Finding Efficiency \\
\hline \hline$K$ or $\pi$ & $(97.9 \pm 0.6 \pm 0.2) \%$ \\
\hline$K_{s}^{0}$ & $(89.5 \pm 0.9 \pm 0.4) \%$ \\
\hline$\phi(1020)$ & $(90.4 \pm 0.9 \pm 1.9) \%$ \\
\hline \hline
\end{tabular}

Table 4.9: Track finding efficiencies for the different strange mesons. The first uncertainties are statistical and the second are systematic.

of the Monte Carlo calculation. Thus the efficiency values as determined with the use of the second method should be reliable.

Using this second procedure, the CTC pattern recognition efficiency was determined to be $(89.5 \pm 0.9) \%$ for both $K_{s}^{0}$ daughter tracks, $(90.4 \pm 0.9) \%$ for both $\phi(1020)$ daughter tracks and $(97.9 \pm 0.6) \%$ for kaon and pion tracks in $K^{*}(892)^{0}$ events. The uncertainties for the efficiencies are statistical.

Since the second method gives the more unbiased estimate of the CTC pattern recognition efficiency, it is the method that is used in the determination of the ratios of branching fractions. The systematic uncertainties associated with the CTC patterning finding efficiencies were taken to be half of the difference of the efficiencies as determined by the two methods. The resulting efficiencies are summarized in Table 4.9. 


\subsection{Confidence Level Requirement Efficiencies}

The efficiency calculations for some of the confidence level requirements have already been described in Sections 3.2 to 3.4 using inclusive samples of events. Although the confidence level distributions were not all uniform as expected, the measured efficiencies for the criteria using the fit results were close to the expected values. Because the statistics for the reconstructed bottom mesons are very poor, the methods that were used to evaluate these efficiencies for the inclusive channels cannot be used for the exclusive bottom meson decays. Instead, the values obtained from the inclusive studies are used. Because of this, it is important to verify that the confidence level distributions are consistent between the inclusive events and bottom meson candidates. The sideband subtracted confidence level distributions for the exclusive decays are shown in Figures 4.7 to 4.11, and compare well to those observed for the inclusive channels. The efficiencies for the inclusive calculations are summarized in Table 4.10.

For the prompt decays, such as the $J / \psi$ and $\phi(1020)$ decays, the efficiencies are similar, so they should cancel out in a ratio. A systematic uncertainty on the ratio of efficiencies for the confidence level requirement of the prompt components of the bottom meson vertex constraint is taken to be half the difference between the values obtain for the inclusive $J / \psi$ and $\phi(1020)$ meson decays. The equivalent $K_{s}^{0}$ values are taken from Table 4.10, which summarizes the efficiencies for the confidence level requirements using the inclusive decays.

To check for any $P_{T}$ dependence, the $K_{s}^{0}$ efficiencies were also calculated for events with a minimum transverse momentum requirement of $2 \mathrm{GeV} / c$ on the dipion system. Both the low $P_{T}$ and high $P_{T}$ values are consistent with each other. Therefore, the average efficiency values of $93.8 \%$ and $98.3 \%$ were used for the vertex only and mass and vertex constraints for the respective $K_{s}^{0}$ requirements. The systematic uncertainty is taken to be half of the difference. 


\begin{tabular}{|l|c|c|}
\hline \hline Decay & Constraint & Efficiency \\
\hline \hline$J / \psi \rightarrow \mu^{+} \mu^{-}$ & Vertex Only & $97.6 \pm 0.5 \%$ \\
\hline$\phi(1020) \rightarrow K^{+} K^{-}$ & Vertex Only & $96.5 \pm 0.3 \%$ \\
\hline$K_{s}^{0} \rightarrow \pi^{+} \pi^{-}$ & Vertex Only & $93.1 \pm 1.7 \%$ \\
\hline$J / \psi \rightarrow \mu^{+} \mu^{-}$ & Mass and Vertex & $97.5 \pm 0.4 \%$ \\
\hline$K_{s}^{0} \rightarrow \pi^{+} \pi^{-}$ & Mass and Vertex & $97.7 \pm 0.3 \%$ \\
\hline$K_{s}^{0} \rightarrow \pi^{+} \pi^{-}$ & Vertex Only (high $\left.P_{T}\right)$ & $94.5 \pm 1.5 \%$ \\
\hline$K_{s}^{0} \rightarrow \pi^{+} \pi^{-}$ & Mass and Vertex (high $\left.P_{T}\right)$ & $98.8 \pm 0.3 \%$ \\
\hline \hline
\end{tabular}

Table 4.10: Summary of efficiencies for confidence level requirements using the inclusive modes.
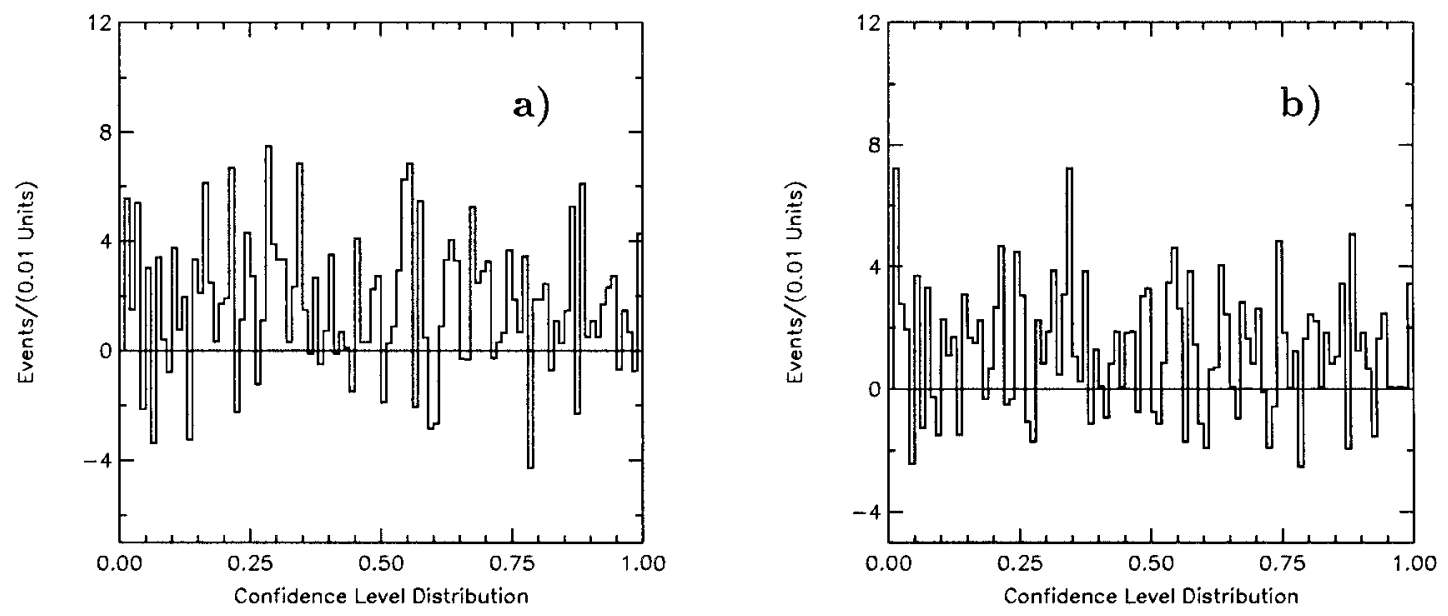

Figure 4.7: The confidence level distributions for the $B^{+} \rightarrow J / \psi K^{+}$mass and vertex fit. The momentum requirements on the strange and bottom mesons were 1.5 and $8.0 \mathrm{GeV} / c$ for (a) and 2.0 and $8.0 \mathrm{GeV} / c$ for (b). Both distributions were sideband subtracted. 

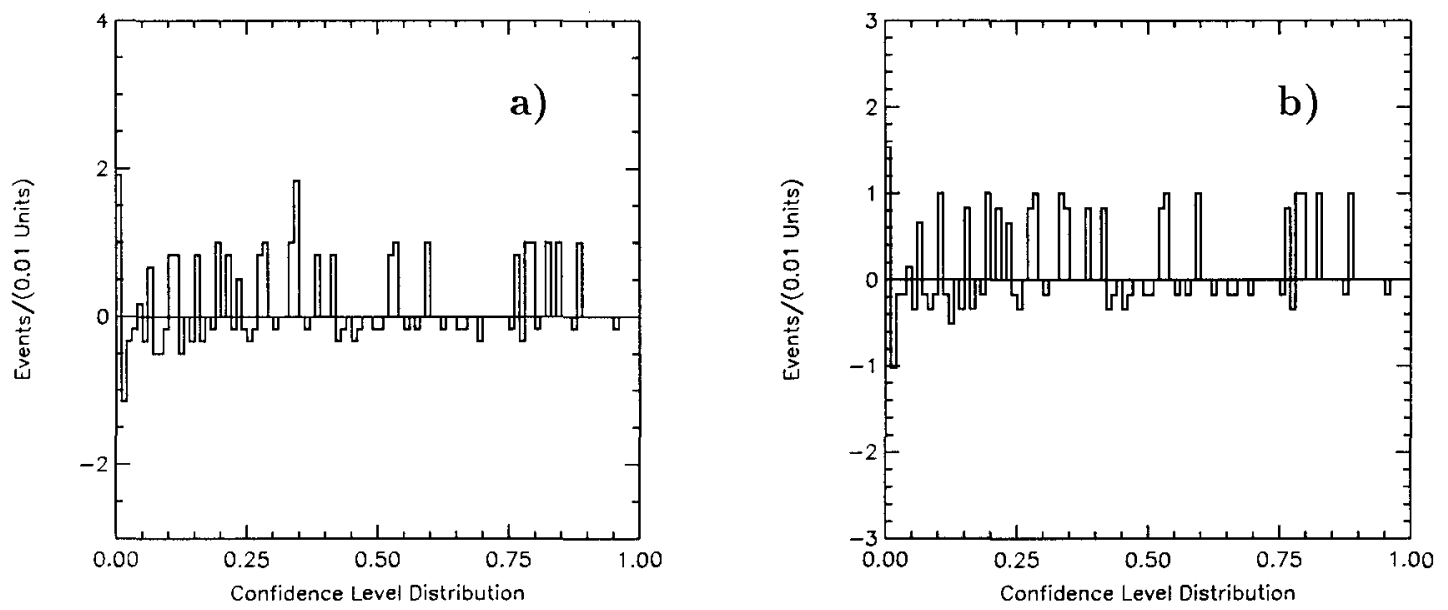

Figure 4.8: The confidence level distributions for the $B^{+} \rightarrow J / \psi K^{*}(892)^{+}$mass and vertex fit. The momentum requirements on the strange and bottom mesons were 2.0 and $6.0 \mathrm{GeV} / c$ for (a) and 2.0 and $8.0 \mathrm{GeV} / c$ for (b). Both distributions were sideband subtracted.
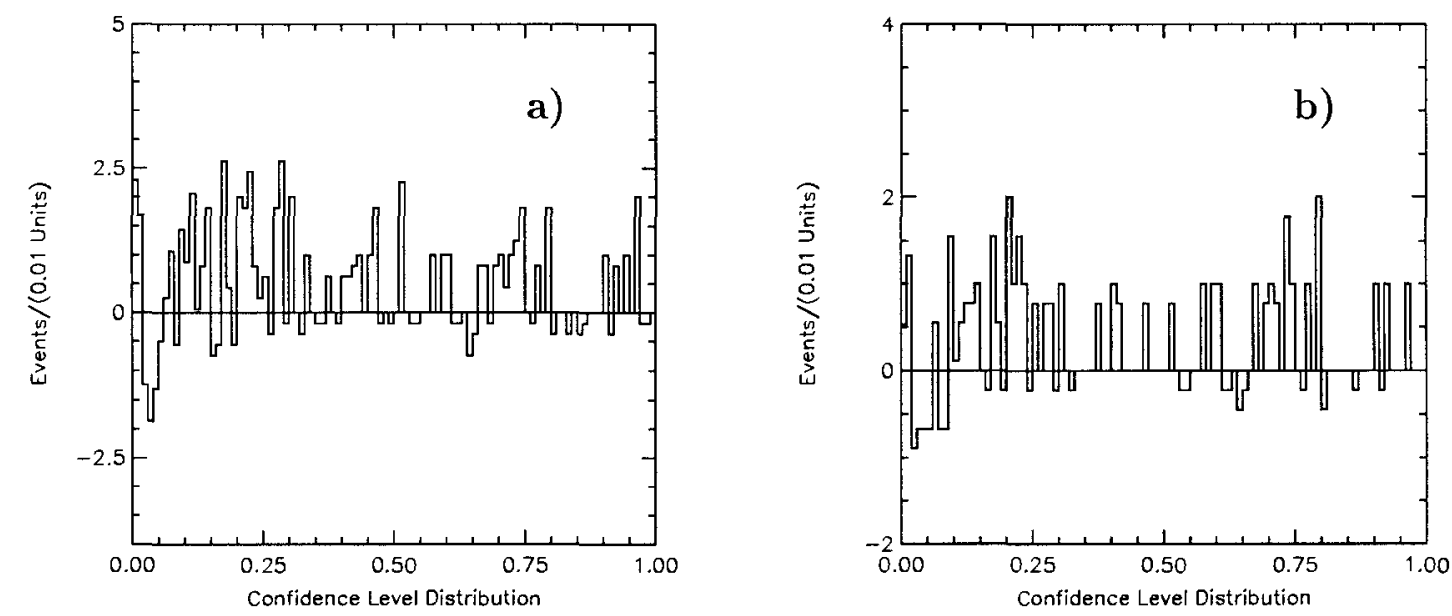

Figure 4.9: The confidence level distributions for the $B^{0} \rightarrow J / \psi K_{s}^{0}$ mass and vertex fit. The momentum requirements on the strange and bottom mesons were 1.5 and $6.0 \mathrm{GeV} / c$ for (a) and 2.0 and $8.0 \mathrm{GeV} / c$ for $(\mathrm{b})$. Both distributions were sideband subtracted. 


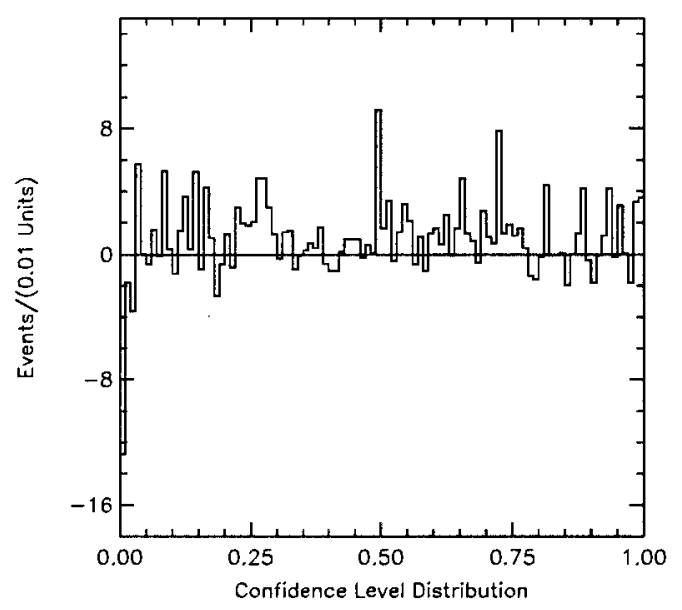

Figure 4.10: The confidence level distribution for the $B^{0} \rightarrow J / \psi K^{*}(892)^{0}$ mass and vertex fit. The momentum requirements on the strange and bottom mesons were 2.0 and $8.0 \mathrm{GeV} / c$. The distribution was sideband subtracted.
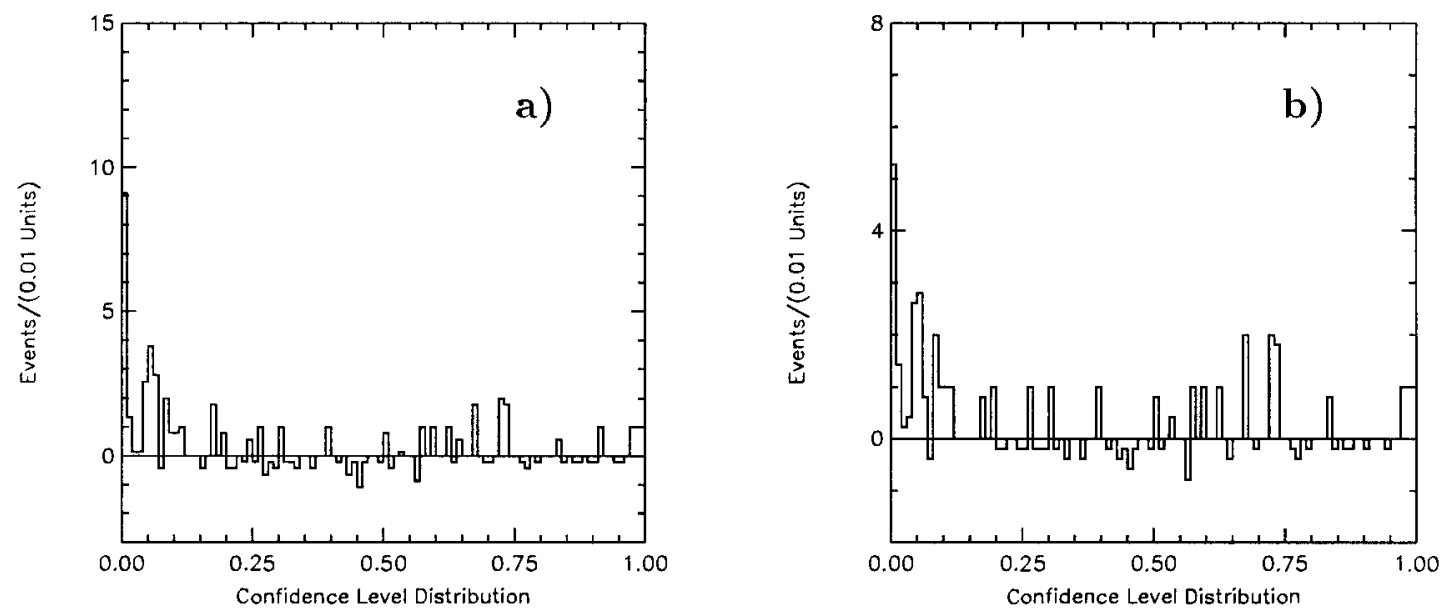

Figure 4.11: The confidence level distributions for the $B_{s}^{0} \rightarrow J / \psi \phi(1020)$ mass and vertex fit. The momentum requirements on the strange and bottom mesons were 1.5 and $8.0 \mathrm{GeV} / c$ for (a) and 2.0 and $8.0 \mathrm{GeV} / c$ for (b). Both distributions were sideband subtracted. 


\section{Chapter 5}

\section{Discussion of Systematic Uncertainties}

The advantage of a calculation of ratios of efficiencies is that some systematic uncertainties will cancel out in a ratio. Cancellations will occur for both theoretical uncertainties and uncertainties on measured quantities that are common in the ratios of efficiencies. Because the statistical uncertainties for all of the five reconstructed bottom mesons decays are greater than $10 \%$, only systematic uncertainties greater than $1 \%$ are considered. Thus, an uncertainty such as that on the world average branching fraction for the $K_{s}^{0} \rightarrow \pi^{+} \pi^{-}$of $0.41 \%$ [7] is not included as a systematic uncertainty on the ratio of branching fractions involving the $K_{s}^{0}$ meson. This is not the case for the $\phi(1020) \rightarrow K^{+} K^{-}$decay as the uncertainty on the world average branching fraction is $1.8 \%$.

\subsection{Muon and $J / \psi$ Requirements}

Since the muon and $J / \psi$ requirements are similar in all of the five bottom mesons decays, the ratio of the corresponding efficiencies should be very close to 1 . The level 1 and level 2 dimuon trigger efficiencies do not necessarily cancel in the ratios as there are different kinematic requirements on the bottom mesons. To verify that the efficiencies for the level 1 and level 2 dimuon triggers for the five decays are equal, the Monte Carlo calculations described in Section 4.1 were redone with two different trigger parameterizations. Both the level 1 and level 2 trigger parameterizations were changed by one standard deviation. The efficiencies associated with the default parameterizations and those corresponding to changes of one standard deviation of 


\begin{tabular}{|l|c|c|c|c|}
\hline \hline Decay & Lower L1 & Higher L1 & Lower L2 & Higher L2 \\
\hline \hline$B^{+} \rightarrow J / \psi K^{+}$ & $94.3 \pm 0.3$ & $104.8 \pm 0.3$ & $99.0 \pm 0.1$ & $100.8 \pm 0.2$ \\
\hline$B^{+} \rightarrow J / \psi K^{*}(892)^{+}$ & $94.7 \pm 0.7$ & $104.6 \pm 0.7$ & $99.7 \pm 0.3$ & $101.4 \pm 0.4$ \\
\hline$B^{0} \rightarrow J / \psi K_{s}^{0}$ & $96.5 \pm 0.5$ & $105.8 \pm 0.9$ & $99.5 \pm 0.4$ & $103.4 \pm 0.5$ \\
\hline$B^{0} \rightarrow J / \psi K^{*}(892)^{0}$ & $96.5 \pm 0.3$ & $105.9 \pm 0.4$ & $99.7 \pm 0.2$ & $103.4 \pm 0.3$ \\
\hline$B_{s}^{0} \rightarrow J / \psi \phi(1020)$ & $97.3 \pm 0.2$ & $105.1 \pm 0.3$ & $99.2 \pm 0.2$ & $101.9 \pm 0.3$ \\
\hline \hline
\end{tabular}

Table 5.1: Relative percentage change in acceptance with the use of various trigger parameterizations. The higher L1 and L2 columns represent higher level 1 and level 2 parameterizations shown in Figure 3.9. The lower L1 and L2 columns represent lower level 1 and level 2 parameterizations shown in Figure 3.9.

the level 1 and level 2 efficiency curves are shown in Figure 3.9.

The effects of the various triggers parameterizations are summarized in Table 5.1. From Table 5.1 one can see that at most there are a few variations of the order $1 \%$ that are within the statistical uncertainties of the measurements. Thus, the ratios of efficiencies for the five bottom mesons decays due to the level 1 and level 2 dimuon trigger is taken to be one with negligible systematic uncertainties.

\subsection{Proper Decay Length Requirements}

The effect of the proper decay length requirement on the bottom mesons and $K_{s}^{0}$ mesons were already discussed in Section 4.2.

For the bottom meson proper decay length requirement, the systematic uncertainties were evaluated by taking the maximum difference of the efficiencies as calculated with the Gaussian-smeared proper decay length distributions using the world average values perturbed by one standard deviation.

The systematic uncertainty on the $K_{s}^{0}$ proper decay length requirement was taken to be the standard deviation of the four measured efficiencies using the two methods that were described in Section 4.2.

In summary, the efficiencies and systematic uncertainties for the proper decay length requirements for the bottom mesons and $K_{s}^{0}$ mesons are listed in Table 5.2. 


\begin{tabular}{|l|c|}
\hline \hline Decay & Efficiency \\
\hline \hline$B^{+} \rightarrow J / \psi K^{+}$ & $(90.0 \pm 0.5) \%$ \\
\hline$B^{+} \rightarrow J / \psi K^{*}(892)^{+}$ & $(88.0 \pm 0.6) \%$ \\
\hline$B^{0} \rightarrow J / \psi K_{s}^{0}$ & $(87.6 \pm 0.7) \%$ \\
\hline$B^{0} \rightarrow J / \psi K^{*}(892)^{0}$ & $(89.3 \pm 0.6) \%$ \\
\hline$B_{s}^{0} \rightarrow J / \psi \phi(1020)$ & $(88.4 \pm 2.0) \%$ \\
\hline$K_{s}^{0} \rightarrow \pi^{+} \pi^{-}$ & $(95.8 \pm 0.7) \%$ \\
\hline \hline
\end{tabular}

Table 5.2: Efficiencies and systematic uncertainties for the proper decay length requirements (positive $c \tau(B), c \tau\left(K_{s}^{0}\right)>1 \mathrm{~mm}$ ).

\begin{tabular}{|l|c|c|}
\hline \hline Decay & Less Long. & More Long. \\
\hline \hline$B^{+} \rightarrow J / \psi K^{*}(892)^{+}$ & $101.2 \pm 0.2 \%$ & $97.3 \pm 0.4 \%$ \\
\hline$B^{0} \rightarrow J / \psi K^{*}(892)^{0}$ & $100.7 \pm 0.2 \%$ & $97.5 \pm 0.4 \%$ \\
\hline$B_{s}^{0} \rightarrow J / \psi \phi(1020)$ & $101.9 \pm 0.2 \%$ & $97.8 \pm 0.4 \%$ \\
\hline \hline
\end{tabular}

Table 5.3: Relative percentage change in acceptance with the use of the various amounts of longitudinal polarization, as given in Table 4.1.

\subsection{Helicity Distribution}

Pseudoscalar to vector-vector decays can have a mixture of polarization amplitudes. As mentioned in Section 4.1, the CLEO Monte Carlo program [57] was used to decay the daughter particles with various amounts of longitudinal polarization, as given in Table 4.1.

For the vector-vector decays, the relative percentage change in acceptance with the use of the various amounts of longitudinal polarization are shown in Table 5.3.

Because the relative percentage changes in acceptance as a function of longitudinal polarization is not symmetric, the average maximum difference of $2.5 \%$ is taken to be the systematic uncertainty on the acceptance values.

\subsection{Confidence Level Requirements}

The confidence level requirements were already shown to be highly efficient for the inclusive $J / \psi, K_{s}^{0}$ and $\phi(1020)$ decays. 
Using the inclusive modes, it was shown that the average efficiency for the vertex constrained confidence level requirements applied to short-lived mesons $(J / \psi$ and $\phi(1020))$ was approximately $97 \%$. It is therefore reasonable to believe that the efficiencies for a vertex constrained confidence level requirements applied to the short-lived particles like bottom mesons would be equal. Nevertheless, a $1 \%$ systematic uncertainty is assigned for the ratio of efficiencies due to the confidence level requirement on the bottom meson vertex constraint fit. This $1 \%$ systematic uncertainty is estimated from the difference in efficiencies for the confidence level requirements for the two inclusive short-lived meson values.

The efficiencies of the confidence level requirements on the long-lived $K_{s}^{0}$ candidates were smaller than the efficiencies on the short-lived mesons but not inconsistent when one considers the uncertainties listed in Table 4.10. The efficiencies and their associated systematic uncertainty were determined to be $(93.8 \pm 1.7) \%$ for the vertex constraint and $(98.3 \pm 0.6) \%$ with the additional mass constraint.

\subsection{Charged Kaon Decay in Flight}

A certain number of charged kaons will decay before they reach the outer CTC can. Some of these kaons are nevertheless successfully reconstructed. The number of kaons that decayed before reaching the outer limit of the CTC chamber that were not successfully reconstructed was determined with the use of the Monte Carlo calculation described in Section 4.1.

For the decay $B^{+} \rightarrow J / \psi K^{+}$, it was found that $4 \%$ of the kaons that were to go through the CTC decayed inside the CTC. Of those kaons that decayed inside the CTC volume, $40 \%$ were found. A track was considered found using the same criteria as mentioned in Section 4.3. For the decay $B^{0} \rightarrow J / \psi K^{*}(892)^{0}, 5.5 \%$ of the kaons decayed inside the CTC, and $46 \%$ of them were found. For the decay, $B_{s}^{0} \rightarrow J / \psi \phi(1020), 6.5 \%$ of the kaons decayed and $55 \%$ were found.

The detector simulation used in the Monte Carlo calculation should accurately estimate the reconstruction efficiency of kaons that decay. A systematic uncertainty equal to the number of unreconstructed tracks is assigned $(3 \%)$. 


\begin{tabular}{|l|c|c|}
\hline \hline Decay & Set 1 & Set 2 \\
\hline \hline$B^{+} \rightarrow J / \psi K^{+}$ & $(113.6 \pm 0.8) \%$ & $(109.2 \pm 0.7) \%$ \\
\hline$B^{+} \rightarrow J / \psi K^{*}(892)^{+}$ & $(104.5 \pm 0.5) \%$ & $(103.5 \pm 0.4) \%$ \\
\hline$B^{0} \rightarrow J / \psi K^{0}$ & $(104.1 \pm 0.4) \%$ & $(103.8 \pm 0.3) \%$ \\
\hline$B^{0} \rightarrow J / \psi K^{*}(892)^{0}$ & $(107.0 \pm 0.6) \%$ & $(106.0 \pm 0.6) \%$ \\
\hline$B_{s}^{0} \rightarrow J / \psi \phi(1020)$ & $(106.9 \pm 0.5) \%$ & $(105.3 \pm 0.5) \%$ \\
\hline \hline
\end{tabular}

Table 5.4: Relative percentage changes of the acceptance with the use of different parameterizations for the bottom quark differential production cross section with different momentum requirements on the bottom and strange mesons. The first set was calculated with $\mu=\mu_{0} / 4$ and $m_{b}=4.5 \mathrm{GeV} / c^{2}$ and the second set was calculated with $\mu=2 \mu_{0}$ and $m_{b}=5.0 \mathrm{GeV} / c^{2}$.

\subsection{Differential Bottom Quark Production Cross Section}

\subsubsection{Shape Effects}

Systematic uncertainties in the shape of the bottom quark differential production cross section as a function of the bottom quark transverse momentum must also be taken into account. The input bottom quark differential production cross section that was used in the default Monte Carlo calculation described in Section 4.1 was varied by changing $\mu$ from $\mu_{0}$ to $\mu_{0} / 4$ and $m_{b}$ to $4.5 \mathrm{GeV} / c^{2}$ and by changing $\mu$ from $\mu_{0}$ to $2 \mu_{0}$ and $m_{b}$ to $5.0 \mathrm{GeV} / c^{2}$. The change in the parameters represent the uncertainty in the theoretical prediction of the bottom quark differential production cross section as given by Reference [12]. The relative percentage changes of the Monte Carlo acceptance calculation with the use of these two sets of parameters are listed in Tables 5.4 and 5.5. The systematic uncertainty on the shape of the bottom quark differential production cross section is taken to be half the maximum difference between the two sets of calculations. For these cases, where half the difference is less than the statistical uncertainty, a $1 \%$ systematic uncertainty is assigned.

\subsubsection{Effects of Higher Spin States}

To determine the effect of the production of excited bottom hadron states on the efficiency to detect the lowest lying bottom hadron states produced through decays of 


\begin{tabular}{|l|c|c|}
\hline \hline Decay & Set 1 & Set 2 \\
\hline \hline$B^{+} \rightarrow J / \psi K^{+}$ & $(114.6 \pm 0.9) \%$ & $(109.5 \pm 0.7) \%$ \\
\hline$B^{+} \rightarrow J / \psi K^{*}(892)+$ & $(106.4 \pm 0.6) \%$ & $(106.0 \pm 0.4) \%$ \\
\hline$B^{0} \rightarrow J / \psi K^{0}$ & $(108.0 \pm 0.6) \%$ & $(107.8 \pm 0.6) \%$ \\
\hline$B^{0} \rightarrow J / \psi K^{*}(892)^{0}$ & $(107.0 \pm 0.6) \%$ & $(106.0 \pm 0.6) \%$ \\
\hline$B_{s}^{0} \rightarrow J / \psi \phi(1020)$ & $(111.2 \pm 0.7) \%$ & $(104.9 \pm 0.5) \%$ \\
\hline \hline
\end{tabular}

Table 5.5: Relative percentage changes of the acceptance with the use of different parameterizations for the bottom quark differential production cross section with similar momentum requirements on the bottom and strange mesons. The first set was calculated with $\mu=\mu_{0} / 4$ and $m_{b}=4.5 \mathrm{GeV} / c^{2}$ and the second set was calculated with $\mu=2 \mu_{0}$ and $m_{b}=5.0 \mathrm{GeV} / c^{2}$.

\begin{tabular}{|l|c|c|c|}
\hline \hline Mesons/Flavor & $\mathrm{U}_{\mathrm{p}}$ & Down & Strange \\
\hline \hline$B$ & 5278.7 & 5279.0 & 5368.0 \\
\hline$B^{*}$ & 5324.8 & 5324.8 & 5414.0 \\
\hline$B_{1}$ & 5730.0 & 5730.0 & 5860.0 \\
\hline$B_{0}^{*}$ & 5680.0 & 5680.0 & 5810.0 \\
\hline$B_{1}^{*}$ & 5780.0 & 5780.0 & 5910.0 \\
\hline$B_{2}^{*}$ & 5830.0 & 5830.0 & 5960.0 \\
\hline \hline
\end{tabular}

Table 5.6: Invariant masses of the various bottom mesons that were used in the PYTHIA Monte Carlo calculation of detection efficiencies. All the masses are in units of $\mathrm{MeV} / \mathrm{c}^{2}$.

excited bottom hadrons, the standard Monte Carlo calculation was repeated with the inclusion of some fraction of excited states. The bottom quark differential production cross section was modeled using the PYTHIA generator [59]. In the PYTHIA calculation, all the known bottom mesons masses were set to the PDG [7] mass values except for the $B_{s}^{0}$ meson mass, which was shifted down by $0.11 \mathrm{GeV} / c^{2}$ to $5.368 \mathrm{GeV} / c^{2}$. The remaining $s \bar{b}$ masses were also shifted down by $0.11 \mathrm{GeV} / c^{2}$. The bottom meson masses that were used in the Monte Carlo calculation are summarized in Table 5.6. In addition, the bottom quark mass was set to $4.75 \mathrm{GeV} / c^{2}$ and the minimum bottom quark transverse momentum was required to be greater than $5.0 \mathrm{GeV} / c$.

The relative production rates of the orbitally excited $(L=1)$ bottom hadron 


\begin{tabular}{|l|c|}
\hline \hline Decay & Change in Acceptance \\
\hline \hline$B^{+} \rightarrow J / \psi K^{+}$ & $2.7 \%$ \\
\hline$B^{+} \rightarrow J / \psi K^{*}(892)^{+}$ & $2.0 \%$ \\
\hline$B^{0} \rightarrow J / \psi K^{0}$ & $1.1 \%$ \\
\hline$B^{0} \rightarrow J / \psi K^{*}(892)^{0}$ & $1.5 \%$ \\
\hline$B_{s}^{0} \rightarrow J / \psi \phi(1020)$ & $3.7 \%$ \\
\hline \hline
\end{tabular}

Table 5.7: Relative percentage changes in geometrical acceptance with the inclusion of excited bottom hadrons.

states was set to $30 \%$ for all of the bottom hadron flavors. This is in accordance with the recent DELPHI and ALEPH measurements [30,31]. The remaining $70 \%$ of the states were produced with zero orbital angular momentum. For a given orbital angular momentum state, the bottom mesons were produced in different spin states with the probability for a given spin state determined by spin counting statistics. These relative production rates have been recently confirmed by several LEP experiments $[31,60,61]$.

The effect of the inclusion of the excited states on the Monte Carlo calculated acceptances is shown in Table 5.7. As one can see from the table, the effect of the presence of excited states on the geometric acceptances is minimal. The observed variations in acceptance between the standard calculation and this one were used as the systematic uncertainties due to the effect of higher spin resonances. 


\section{Chapter 6}

\section{Ratios of Branching Fractions Times Ratios of $b$ Quark Fragmentation Fractions}

\subsection{Calculation of Matrix Elements}

The ratio of branching fractions times fragmentation fractions can be determined by measuring the ratio of observed events divided by the ratio of the corresponding detection efficiencies. For the five decays being studied in this analysis, listed in Equation 1.15, this amounts to determining the matrix elements shown in Equation 1.16. Each matrix element is in turn determined with the use of Equation 1.14. For example, the $(2,1)$ matrix element corresponds to the following equation

$$
\frac{\mathcal{B}\left(B^{+} \rightarrow J / \psi K^{*}(892)^{+}\right)}{\mathcal{B}\left(B^{+} \rightarrow J / \psi K^{+}\right)} \cdot \frac{\gamma_{u}}{\gamma_{u}}=\frac{N\left(B^{+} \rightarrow J / \psi K^{*}(892)^{+}\right)}{N\left(B^{+} \rightarrow J / \psi K^{+}\right)} \cdot \frac{\epsilon\left(J / \psi, K^{+}, B^{+}\right)}{\epsilon\left(J / \psi, K^{*}(892)^{+}, B^{+}\right)} .
$$

The number of reconstructed events for the various decays are listed in Tables 3.8 and 3.9 and the various detection efficiencies are listed in Tables 4.3 through 4.10. Since efficiencies for all the cuts that were applied were individually determined in the same order as the application of the cuts, any correlations between the efficiencies values are expected to be small.

The number of events and the efficiencies that do not cancel out in Equation 6.1 are summarized in Table 6.1. Replacing the total efficiency terms given in Equation 6.1 with the individual efficiency terms gives 


\begin{tabular}{|l|c|}
\hline \hline Variable & Value \\
\hline \hline $\mathrm{N}\left(B^{+} \rightarrow J / \psi K^{+}\right)$ & $153.5 \pm 18.9$ \\
\hline $\mathrm{N}\left(B^{+} \rightarrow J / \psi K^{*}(892)^{+}\right)$ & $21.3 \pm 6.1$ \\
\hline$\epsilon_{M C}\left(B^{+} \rightarrow J / \psi K^{+}\right)$ & $0.663 \%$ \\
\hline$\epsilon_{M C}\left(B^{+} \rightarrow J / \psi K^{*}(892)^{+}\right)$ & $0.0619 \%$ \\
\hline$\epsilon_{c \tau}(B)\left(B^{+} \rightarrow J / \psi K^{+}\right)$ & $(90.0 \pm 0.5) \%$ \\
\hline$\epsilon_{c \tau(B)}\left(B^{+} \rightarrow J / \psi K^{*}(892)^{+}\right)$ & $(88.0 \pm 0.6) \%$ \\
\hline$\epsilon_{c \tau\left(K_{s}^{0}\right)}$ & $(95.8 \pm 0.7) \%$ \\
\hline$\epsilon\left(C . L . K_{s}^{0}\right.$ vertex) & $(93.8 \pm 1.7) \%$ \\
\hline$\epsilon\left(C . L . K_{s}^{0}\right.$ mass and vertex) & $(98.3 \pm 0.6) \%$ \\
\hline$\epsilon\left(K_{s}^{0}\right.$ tracking) & $(89.5 \pm 1.0) \%$ \\
\hline Helicity (syst) & $\pm 2.5 \%$ \\
\hline Monte Carlo (syst) & $\pm 5 \%$ \\
\hline$C . L .(B$ vertex) (syst) & $\pm 1 \%$ \\
\hline Decay of Kaon (syst) & $\pm 3 \%$ \\
\hline$P_{T}$ spectrum (syst) & $\pm 4.6 \%$ \\
\hline Effect of Excited states $\left(B^{+} \rightarrow J / \psi K^{+}\right)($syst) & $\pm 2.7 \%$ \\
\hline Effect of Excited states $\left(B^{+} \rightarrow J / \psi K^{*}(892)^{+}\right)$(syst) & $\pm 2.0 \%$ \\
\hline \hline
\end{tabular}

Table 6.1: Numbers that go into the calculation of the matrix elements $(1,2)$ and $(2,1)$ with different momentum cuts on the bottom and strange mesons. 


$$
\begin{aligned}
\frac{\epsilon\left(J / \psi, K^{+}, B^{+}\right)}{\epsilon\left(J / \psi, K^{*}(892)^{+}, B^{+}\right)}= & \frac{\epsilon_{M C}\left(B^{+} \rightarrow J / \psi K^{+}\right)}{\epsilon_{M C}\left(B^{+} \rightarrow J / \psi K^{*}(892)^{+}\right)} \cdot \frac{\epsilon_{c \tau(B)}\left(B^{+} \rightarrow J / \psi K^{+}\right)}{\epsilon_{c \tau(B)}\left(B^{+} \rightarrow J / \psi K^{*}(892)^{+}\right)} \cdot \frac{1}{\epsilon_{c \tau\left(K_{s}^{0}\right)}} \\
& \cdot \frac{1}{\epsilon\left(K_{s}^{0} \text { tracking }\right)} \cdot \frac{1}{\epsilon\left(C . L . K_{s}^{0} \text { vertex }\right)} \cdot \frac{1}{\epsilon\left(C . L . K_{s}^{0} \text { mass and vertex }\right)} .
\end{aligned}
$$

Using the number of observed events and efficiency values from Table 6.1, the ratio of branching fractions for matrix element $(2,1)$ which is given by Equation 6.1 with different transverse momentum requirements was calculated to be

$$
\frac{\mathcal{B}\left(B^{+} \rightarrow J / \psi K^{*}(892)^{+}\right)}{\mathcal{B}\left(B^{+} \rightarrow J / \psi K^{+}\right)}=1.92 \pm 0.60(\text { stat }) \pm 0.17 \text { (syst) }
$$

The statistical uncertainty was calculated from the number of observed events given in the first two rows of Table 6.1 and the systematic uncertainty was calculated from the values taken from the last 13 rows.

In a similar fashion, all of the matrix elements can be calculated with the number of reconstructed events for the various decays taken from Tables 3.8 and 3.9 and the various efficiencies taken from the Tables 4.3 through 4.10. For the remaining matrix elements, the number of events and the efficiencies that do not cancel out are summarized in Tables B.2 through B.20. The resulting matrices with different and similar strange and bottom meson transverse momentum requirements are shown in Equation 6.4 and Equation 6.5, respectively.

$\begin{array}{ccccc}1 & (0.52 \pm 0.16 \pm 0.05) & (0.87 \pm 0.20 \pm 0.07) & (0.63 \pm 0.13 \pm 0.05) & (2.46 \pm 0.74 \pm 0.23) \\ (1.92 \pm 0.60 \pm 0.17) & 1 & (1.68 \pm 0.58 \pm 0.11) & (1.21 \pm 0.40 \pm 0.10) & (4.73 \pm 1.87 \pm 0.46) \\ (1.15 \pm 0.27 \pm 0.10) & (0.60 \pm 0.21 \pm 0.04) & 1 & (0.72 \pm 0.19 \pm 0.05) & (2.82 \pm 0.95 \pm 0.26) \\ (1.59 \pm 0.33 \pm 0.12) & (0.83 \pm 0.27 \pm 0.07) & (1.39 \pm 0.36 \pm 0.10) & 1 & (3.91 \pm 1.26 \pm 0.35) \\ (0.41 \pm 0.12 \pm 0.04) & (0.21 \pm 0.08 \pm 0.02) & (0.35 \pm 0.12 \pm 0.03) & (0.26 \pm 0.08 \pm 0.02) & 1\end{array}$

$$
\begin{array}{ccccc}
1 & (0.48 \pm 0.15 \pm 0.04) & (0.89 \pm 0.23 \pm 0.07) & (0.57 \pm 0.12 \pm 0.04) & (2.16 \pm 0.65 \pm 0.20) \\
(2.08 \pm 0.67 \pm 0.18) & 1 & (1.84 \pm 0.68 \pm 0.12) & (1.20 \pm 0.41 \pm 0.09) & (4.49 \pm 1.81 \pm 0.45) \\
(1.13 \pm 0.29 \pm 0.09) & (0.54 \pm 0.20 \pm 0.03) & 1 & (0.65 \pm 0.18 \pm 0.05) & (2.44 \pm 0.86 \pm 0.23) \\
(1.74 \pm 0.37 \pm 0.13) & (0.84 \pm 0.28 \pm 0.07) & (1.54 \pm 0.43 \pm 0.11) & 1 & (3.76 \pm 1.21 \pm 0.34) \\
(0.46 \pm 0.14 \pm 0.04) & (0.22 \pm 0.09 \pm 0.02) & (0.41 \pm 0.15 \pm 0.04) & (0.27 \pm 0.09 \pm 0.02) & 1
\end{array}
$$

By comparing the matrix elements in Equations 6.4 and 6.5, one can see that there are no obvious biases using either set of transverse momentum requirements on the bottom and strange mesons. Since the matrix elements using the different transverse momentum requirements on the strange and bottom mesons results in 
smaller uncertainties (statistical and systematic combined in quadrature), it is the preferred matrix to use.

In order to calculate ratios of branching fractions, one must still factor out the ratio of fragmentation fractions. Following the arguments discussed in Section 1.4, the assumption $\gamma_{u}=\gamma_{d}$ is made.

The value of $\gamma_{s}$ with respect to $\gamma_{u}$ and $\gamma_{d}$ has been measured using many methods based on different assumptions. Table 6.2 contains a summary of some of the measured results taken from References [62, 63, 64, 65, 66, 67, 68, 69, 70, 71, 72]. From Table 6.2 , one can see that the general trend indicates that $\gamma_{s} / \gamma_{u, d}$ is between 0.27 and 0.40 .

One reasonable choice for $\gamma_{s} / \gamma_{u, d}$ is the average strangeness suppression value as calculated in Reference [72] of $\gamma_{s} / \gamma_{u, d}=0.29 \pm 0.015$. It should be noted that this value does not include the recent ALEPH [71] values. Because the $\gamma_{s} / \gamma_{u, d}$ ratio might have a slight energy dependence, an alternative choice would be to use the value of $\gamma_{s} / \gamma_{u, d}$ of $(0.40 \pm 0.05)$ measured at $\sqrt{s}=1.8 \mathrm{TeV}$ by the CDF collaboration [69]. It should also be noted that the ratio $\gamma_{s} / \gamma_{u, d}$ is a measurement of the rate of $\bar{s} s$ diquark production over the rate of $\bar{u} u$ or $\bar{d} d$ diquark production. Since the decay of excited bottom hadrons can cause an imbalance of detected hadrons with different spectator quarks, a third and more appropriate value to use is that based on the DELPHI measurement of $f_{s}[70]$ which is the fraction of bottom quarks that hadronize to a $B_{s}^{0}$ meson. Using DELPHI's $f_{s}$ measurement and assuming that $f_{u}=f_{d}$ and $f_{c} \simeq 0$ (given the predictions in Equation 1.13), the ratio of $B_{s}^{0}$ to $B^{+}$or $B^{0}$ production can be calculated as a function of $f_{\text {baryon }}$. Taking the value of $f_{\text {baryon }}=0.08$ which the DELPHI collaboration used in its Monte Carlo efficiency calculations, the ratio of $B_{s}^{0}$ to $B^{+}$or $B^{0}$ production is calculated to be $0.52 \pm 0.35$.

Each of the values described above is based on certain assumptions. Thus, one can use any of the three values described above depending on what assumption one wants to use. Alternatively, one can use the three values as an indication of what the actual value of $\gamma_{s} / \gamma_{u, d}$ or $f_{s} / f_{u, d}$ is by considering the range of the three values. In doing so, the value of 0.40 in the middle of the range $0.27-0.52$ is used with an uncertainty of 0.06 , which is one quarter of the difference between 0.27 and 0.52 .

Starting with the matrix elements given in Equation 6.4 and taking $\gamma_{u}=\gamma_{d}$ and $\gamma_{s} / \gamma_{u, d}=0.40 \pm 0.06$, one obtains matrix elements that only contain ratios of branching fractions. These ratios are given as individual matrix elements in Equation 6.6 


\begin{tabular}{|l|l|l|c|}
\hline \hline Exp. & Description & Relation & Value \\
\hline FNAL-180 & $\sqrt{s}=200 \mathrm{GeV}$ primary mesons only & $\gamma_{s} / \gamma_{u, d}$ & $0.25 \pm 0.03$ \\
\hline FNAL-180 & $\sqrt{s}=200 \mathrm{GeV}$ +nonprimary mesons & $\gamma_{s} / \gamma_{u, d}$ & $0.27 \pm 0.04$ \\
\hline Malhotra & Weighted Average $(1983)$ & $\gamma_{s} / \gamma_{u, d}$ & $0.29 \pm 0.2$ \\
\hline PETRA & $\sqrt{s}=12 \mathrm{GeV}$ & $\gamma_{s} / \gamma_{u, d}$ & $0.37_{-0.21}^{+0.37}$ \\
\hline PETRA & $\sqrt{s}=14 \mathrm{GeV}$ & $\gamma_{s} / \gamma_{u, d}$ & $0.26_{-0.10}^{+0.12}$ \\
\hline PETRA & $\sqrt{s}=22 \mathrm{GeV}$ & $\gamma_{s} / \gamma_{u, d}$ & $0.28_{-0.10}^{+0.12}$ \\
\hline PETRA & $\sqrt{s}=30 \mathrm{GeV}$ & $\gamma_{s} / \gamma_{u, d}$ & $0.29_{-0.11}^{+0.13}$ \\
\hline PETRA & $\sqrt{s}=35 \mathrm{GeV}$ & $\gamma_{s} / \gamma_{u, d}$ & $0.26_{-0.06}^{+0.06}$ \\
\hline PETRA & Average & $\gamma_{s} / \gamma_{u, d}$ & $0.27 \pm 0.03 \pm 0.05$ \\
\hline PEP-4 & $\sqrt{s}=29 \mathrm{GeV}$ from $2 \mathrm{~N}(\phi) / \mathrm{N}\left(K^{* 0}\right)$ & $\gamma_{s} / \gamma_{u, d}$ & $0.37 \pm 0.15 \pm 0.08$ \\
\hline PEP-4 & $\sqrt{s}=29 \mathrm{GeV}$ from N $\left(K^{* 0}\right) / 2 \mathrm{~N}\left(\rho^{0}\right)$ & $\gamma_{s} / \gamma_{u, d}$ & $0.32 \pm 0.09 \pm 0.05$ \\
\hline UA5 $(85)$ & $\sqrt{s}=540 \mathrm{GeV}$ & $\gamma_{s} / \gamma_{u, d}$ & $0.30 \pm 0.03 \pm 0.03$ \\
\hline UA5 $(88)$ & $\sqrt{s}=200 \mathrm{GeV}$ & $\gamma_{s} / \gamma_{u, d}$ & $0.26 \pm 0.03$ \\
\hline UA5 $(88)$ & $\sqrt{s}=546 \mathrm{GeV}$ & $\gamma_{s} / \gamma_{u, d}$ & $0.30 \pm 0.03$ \\
\hline UA5 $(88)$ & $\sqrt{s}=900 \mathrm{GeV}$ & $\gamma_{s} / \gamma_{u, d}$ & $0.29 \pm 0.02$ \\
\hline CLEO & $\sqrt{s}=10.55 \mathrm{GeV}(D$ mesons $)$ & $\gamma_{u}+\gamma_{d}$ & $0.71 \pm 0.06 \pm 0.10$ \\
\hline CDF & $\sqrt{s}=630 \mathrm{GeV}$ & $\gamma_{s} / \gamma_{u, d}$ & $0.4 \pm 0.2$ \\
\hline CDF & $\sqrt{s}=1800 \mathrm{GeV}$ & $\gamma_{s} / \gamma_{u, d}$ & $0.40 \pm 0.05$ \\
\hline UA1 & $\sqrt{s}=630 \mathrm{GeV}$ & $\gamma_{s} / \gamma_{u, d}$ & $0.29 \pm 0.02 \pm 0.01$ \\
\hline UA1 & World Average $(1996)$ & $\gamma_{s} / \gamma_{u, d}$ & $0.29 \pm 0.015$ \\
\hline DELPHI & $\sqrt{s}=91.19 \mathrm{GeV}\left(D_{s}\right.$ rate $)$ & $f_{s}$ & $0.24 \pm 0.08_{-0.09}^{+0.15}$ \\
\hline DELPHI & $\sqrt{s}=91.19 \mathrm{GeV}(\phi-\mu$ events $)$ & $f_{s}$ & $0.18 \pm 0.12 \pm 0.08$ \\
\hline DELPHI & $\sqrt{s}=91.19 \mathrm{GeV}\left(D_{s}-l e p t o n\right.$ events $)$ & $f_{s}$ & $0.17 \pm 0.08 \pm 0.06$ \\
\hline DELPHI & $\sqrt{s}=91.19 \mathrm{GeV}($ Combined $)$ & $f_{s}$ & $0.19 \pm 0.06 \pm 0.08$ \\
\hline ALEPH & $\sqrt{s}=91.19 \mathrm{GeV}$ from $\mathrm{N}\left(K^{* 0}\right) / 2 \mathrm{~N}\left(\rho^{0}\right)$ & $\gamma_{s} / \gamma_{u, d}$ & $0.29 \pm 0.01 \pm 0.05$ \\
\hline ALEPH & $\sqrt{s}=91.19 \mathrm{GeV}$ from $\mathrm{N}\left(K^{* 0}\right) / 2 \mathrm{~N}(\omega)$ & $\gamma_{s} / \gamma_{u, d}$ & $0.39 \pm 0.02 \pm 0.06$ \\
\hline ALEPH & $\sqrt{s}=91.19 \mathrm{GeV}$ from $2 \mathrm{~N}(\phi) / \mathrm{N}\left(K^{* 0}\right)$ & $\gamma_{s} / \gamma_{u, d}$ & $0.29 \pm 0.01 \pm 0.04$ \\
\hline \hline
\end{tabular}

Table 6.2: Various relations for experimental $\gamma$ values. Note that each measurement uses different assumptions. A more complete compilation without the CLEO [62] and LEP [70, 71] measurements can be found in Reference [72]. 
where the first uncertainties are statistical and the second are systematic.

$\begin{array}{ccccc}1 & (0.52 \pm 0.16 \pm 0.05) & (0.87 \pm 0.20 \pm 0.07) & (0.63 \pm 0.13 \pm 0.05) & (0.98 \pm 0.30 \pm 0.11) \\ (1.92 \pm 0.60 \pm 0.17) & 1 & (1.68 \pm 0.58 \pm 0.11) & (1.21 \pm 0.40 \pm 0.10) & (1.89 \pm 0.75 \pm 0.22) \\ (1.15 \pm 0.27 \pm 0.10) & (0.60 \pm 0.21 \pm 0.04) & 1 & (0.72 \pm 0.19 \pm 0.05) & (1.13 \pm 0.38 \pm 0.13) \\ (1.59 \pm 0.33 \pm 0.12) & (0.83 \pm 0.27 \pm 0.07) & (1.39 \pm 0.36 \pm 0.10) & 1 & (1.57 \pm 0.50 \pm 0.17) \\ (1.02 \pm 0.30 \pm 0.11) & (0.53 \pm 0.21 \pm 0.06) & (0.89 \pm 0.30 \pm 0.10) & (0.64 \pm 0.21 \pm 0.07) & 1\end{array}$

A comparison of the ratio of branching fractions can also be made with theoretical predictions for ratios of partial widths $(\Gamma)$ by using the world average lifetimes $(\tau)$ for the bottom mesons [7]. The elements in the matrix shown in Equation 6.6 can be related to the ratio of partial widths through $\frac{\mathcal{B}(i)}{\mathcal{B}(j)}=\frac{\Gamma_{i}}{\Gamma_{j}} \cdot \frac{\tau_{i}}{\tau_{j}}$. A comparison of ratio of branching fractions for two sets of theoretical predictions $[38,39]$ and $[40,41]$ are shown in Figure 6.1.

As can been seen from Figure 6.1 the data points and the theoretical predictions from References [38, 39] are in good agreement. This supports the argument that the factorization ansatz used in the theoretical model for the bottom meson decays was reasonable. It also supports the assumptions that were used in the determination of the form factors which included chiral symmetry, heavy quark symmetries, simple pole dominance of the form factors and the normalization to semileptonic $D$ meson data.

From Figure 6.1 one also sees that the calculations from References [40, 41] do not adequately predict two of the three values for the ratios of branching fractions. However, it should be noted that all of the three data points can be viewed as being in good agreement with the predicted values if $\gamma_{s} / \gamma_{u, d}$ is taken to be between 0.19 and 0.22 . Though this scenerio leds to a good agreement between the ratio of branching fractions, the predictions for the branching fractions for the $B^{+} \rightarrow J / \psi K^{*}(892)^{+}$ and $B^{0} \rightarrow J / \psi K^{*}(892)^{+}$decays still disagree with the world average values [7]. In addition, the measured amount of longitudinal polarization for the $K^{*}(892)^{+}$and $K^{*}(892)^{0}$ mesons in $B^{+} \rightarrow J / \psi K^{*}(892)^{+}$and $B^{0} \rightarrow J / \psi K^{*}(892)^{0}$ decays does not agree with the predicted values $[58,73,74]$.

Though the theoretical predictions for the ratio of branching fractions from References $[38,39]$ are in good agreement with data, no predictions were made for the amount of longitudinal polarization for the various strange mesons. However, their choice of a simple pole dominance for their form factors has been criticized in light of the available polarization data [75]. 


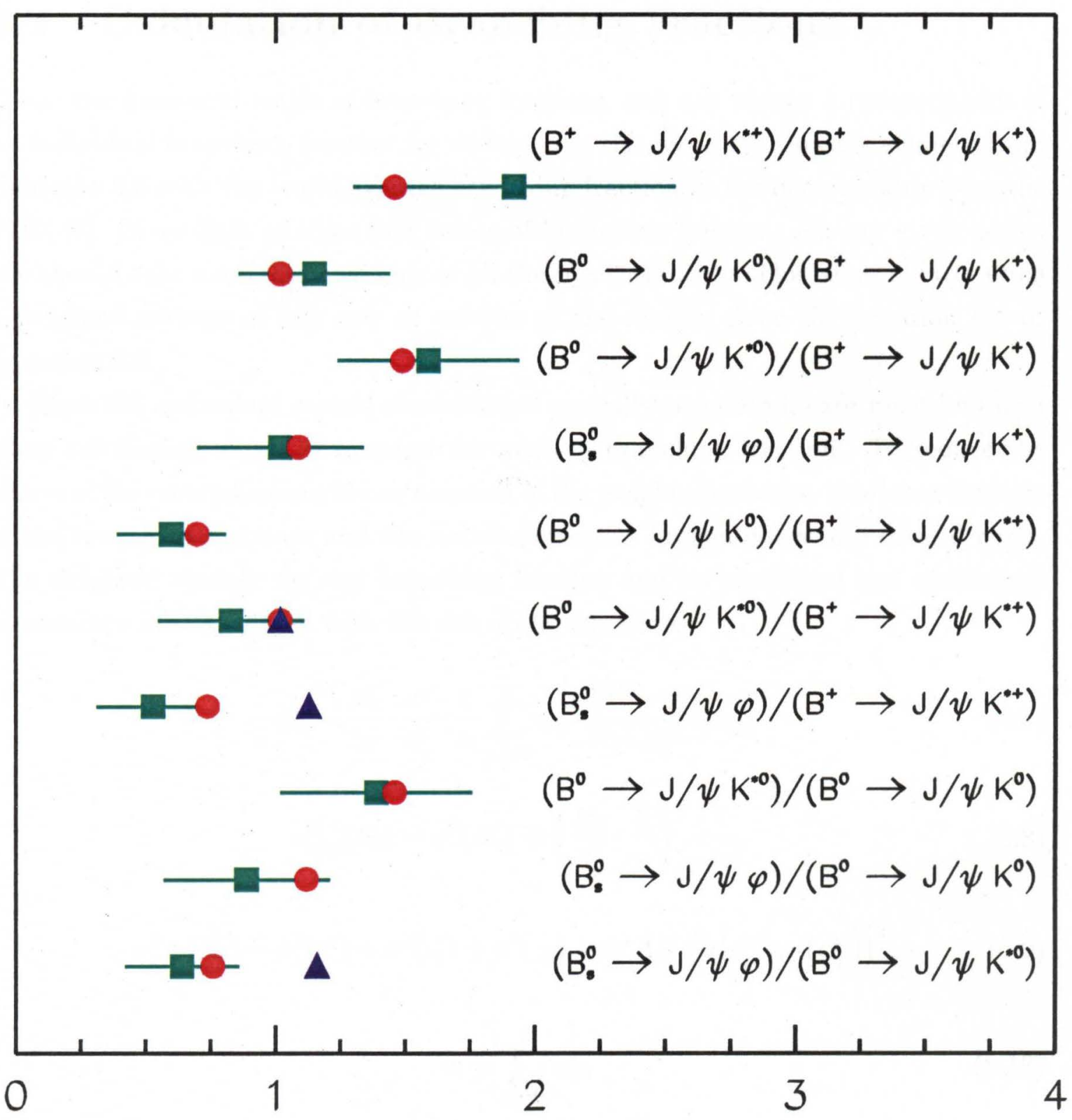

Ratio of branching fractions

Figure 6.1: Comparison of ratio of branching fractions with two sets of theoretical predictions for the ratios of branching fractions. The solid squares are the data points with the statistical and systematic uncertainties added in quadrature. The solid circles are the theoretical predictions taken from [38, 39] scaled with the lifetimes obtained from the PDG. The solid triangles are the theoretical predictions taken from $[40,41]$ scaled with the lifetimes obtained from the PDG. No uncertainties are shown for the theoretical predictions. The assumption that $\gamma_{u}=\gamma_{d}$ was also made in addition to taking $\gamma_{s} / \gamma_{u, d}=0.40 \pm 0.06$. 


\subsection{Calculation of Branching Fractions}

Given the measured ratios of branching fractions, one can obtain a measurement of an individual branching fraction by multiplying the respective matrix element from Equation 6.6 with the world average branching fraction in the denominator from the PDG [7]. Given that one has four ratios of branching fractions for any given decay, one should take a weighted average of all the measurements. This amounts to taking a weighted average of any row or column of the matrix given by Equation 6.4 or Equation 6.6.

Since the individual matrix elements are partially correlated, care must be taken when calculating weighted averages for a given branching fraction. To reduce the effects of the correlations in the calculation of the weighted average, the determination of the branching fractions and the uncertainties are broken down into several parts. The weighted average for any branching fraction and its statistical and systematic uncertainty are calculated with the use of the equations

$$
\begin{gathered}
\mathcal{B}_{i}=\frac{N_{i}}{\gamma_{i}} \cdot \frac{\epsilon^{c}}{\epsilon_{i}^{c}} \cdot \frac{1}{w} \sum_{j, j \neq i}^{5} w_{j} \frac{\mathcal{B}_{j}^{P D G} \cdot \epsilon_{j}^{n c}}{N_{j} \cdot \epsilon_{i j}^{n c}} \cdot \gamma_{j}, \\
\sigma_{\text {stat }}^{2}\left(\mathcal{B}_{i}\right)=\sigma^{2}\left(N_{i}\right)+\left(\frac{N_{i}}{\epsilon_{i}^{c}} \cdot \frac{1}{\gamma_{i}}\right)^{2} \cdot \frac{1}{w}, \\
\sigma_{\text {syst }}^{2}\left(\mathcal{B}_{i}\right)=\sigma^{2}\left(\epsilon^{c}\right)+\sigma^{2}\left(\epsilon_{i}^{c}\right)+\sigma^{2}\left(\gamma_{i}\right)+M A X\left(\sigma\left(\epsilon_{j}^{n c}\right), \sigma\left(\epsilon_{i j}^{n c}\right)\right)^{2}, \\
w=\sum_{j, j \neq i}^{5} w_{j}, \\
w_{j}=\frac{1}{\sigma_{j}^{2}}, \\
\sigma_{j}^{2}=\sigma^{2}\left(\mathcal{B}_{j}^{P D G}\right)+\sigma^{2}\left(N_{j}\right),
\end{gathered}
$$

where the $N_{j}$ 's are the number of observed events for the $j$ 'th decay, the $\mathcal{B}_{j}^{P D G}$ 's are the world average branching fractions, the $\sigma$ 's are the uncertainties, the $\epsilon_{i}^{c}$ 's are the efficiencies that are common to all of the decays, and $\epsilon_{i j}^{n c}$ 's are the efficiencies that are not common to all of the decays. The efficiency uncertainties also include all the 


\begin{tabular}{|l|l|}
\hline \hline Decay & Branching Fraction Value $\left(\times 10^{-3}\right)$ \\
\hline \hline$B^{+} \rightarrow J / \psi K^{+}$ & $(0.82 \pm 0.18($ stat $) \pm 0.07($ syst $))$ \\
\hline$B^{+} \rightarrow J / \psi K^{*}(892)^{+}$ & $(1.73 \pm 0.55($ stat $\pm 0.15($ syst $))$ \\
\hline$B^{0} \rightarrow J / \psi K^{0}$ & $(1.14 \pm 0.27($ stat $) \pm 0.09($ syst $))$ \\
\hline$B^{0} \rightarrow J / \psi K^{*}(892)^{0}$ & $(1.39 \pm 0.32($ stat $) \pm 0.11($ syst $))$ \\
\hline$B_{s}^{0} \rightarrow J / \psi \phi(1020)$ & $(0.37 \pm 0.11($ stat $) \pm 0.04($ syst $)) \cdot\left(\gamma_{u, d} / \gamma_{s}\right)$ \\
\hline$B_{s}^{0} \rightarrow J / \psi \phi(1020)$ & $(0.93 \pm 0.28($ stat $) \pm 0.10($ syst $) \pm 0.14(\gamma))$ \\
\hline \hline
\end{tabular}

Table 6.3: Measured branching fractions values for states involving $J / \psi$ mesons. In calculating the branching fractions it was assumed that $\gamma_{u}=\gamma_{d}$ and $\gamma_{s} / \gamma_{u, d}$ was taken to be $0.40 \pm 0.06$. The third uncertainty in the branching fraction value represents the uncertainty from the value of $\gamma_{s} / \gamma_{u, d}$.

systematic uncertainties. If one assumes that $\gamma_{u}=\gamma_{d}$, the $\gamma_{j}$ value can be taken outside the summation in Equation 6.7 because $\mathcal{B}^{P D G}\left(B_{s}^{0}\right)=0$ (No PDG value) [7].

In determining the weighted average branching fraction for a given decay, all common efficiencies are factored out of the summation in Equation 6.7. The remaining ratios of efficiencies times branching fractions over the number of observed decays are then weighted by $w_{j}$. Since some of the systematic uncertainties are correlated, the weights $w_{j}$ do not include the systematic uncertainties but are taken into account as an uncertainty on the resulting average value. The world average uncertainties on the branching fractions $\mathcal{B}_{j}^{P D G}$ are included in $w_{j}$ because they are dominated by the most recent measurements by the CLEO collaboration [73], which are largely statistical and independent. The systematic uncertainty $\left(\sigma_{\text {syst }}\right)$ for the weighted branching fraction value is then given by a quadrature sum of the systematic uncertainties that are common in all of the decays and the uncommon uncertainties from the decay with the largest quadrature sum of uncommon systematic uncertainties. This sum that is represented by Equation 6.9 is a conservative estimate of the systematic uncertainties because the maximum set of uncommon systematic uncertainties are used. The results from the weighted averages for the five decays are summarized in Table 6.3. The branching fraction measurements for the $B^{+}$and $B^{0}$ mesons are in good agreement with those measured at $e^{+} e^{-}$colliders [73, 76]. The world average branching fractions for the $B^{+}$and $B^{0}$ mesons are shown in Table 6.4. The branching fraction for the $B_{s}^{0} \rightarrow J / \psi \phi(1020)$ decay is the first measurement.

As mentioned in Section 6.1 the choice of $\gamma_{s} / \gamma_{u, d}$ is not unique. One can also 


\begin{tabular}{|l|l|}
\hline \hline Decay & Branching Fraction Value $\left(\times 10^{-3}\right)$ \\
\hline \hline$B^{+} \rightarrow J / \psi K^{+}$ & $(1.02 \pm 0.14)$ \\
\hline$B^{+} \rightarrow J / \psi K^{*}(892)^{+}$ & $(1.7 \pm 0.5)$ \\
\hline$B^{0} \rightarrow J / \psi K^{0}$ & $(0.75 \pm 0.21)$ \\
\hline$B^{0} \rightarrow J / \psi K^{*}(892)^{0}$ & $(1.58 \pm 0.28)$ \\
\hline \hline
\end{tabular}

Table 6.4: World average branching branching fractions values for states involving $J / \psi$ mesons. In calculating the branching fractions it was assumed that $\gamma_{u}=\gamma_{d}=$ $f_{u}=f_{d}$.

\begin{tabular}{|l|l|}
\hline \hline Choice of $\gamma_{s} / \gamma_{u, d}$ & Branching Fraction Value $\left(\times 10^{-3}\right)$ \\
\hline \hline CDF's $(0.40 \pm 0.05)$ & $0.93 \pm 0.28$ (stat) $\pm 0.10($ syst $) \pm 0.12(\gamma)$ \\
\hline UA1's $(0.29 \pm 0.015)$ & $1.28 \pm 0.38$ (stat) $\pm 0.14($ syst $) \pm 0.05(\gamma)$ \\
\hline DELPHI's $(0.52 \pm 0.35)$ & $0.71 \pm 0.21$ (stat) $\pm 0.08($ syst $) \pm 0.48(\gamma)$ \\
\hline \hline
\end{tabular}

Table 6.5: Branching fraction values for the decay $B_{s}^{0} \rightarrow J / \psi \phi(1020)$ as calculated with various $\gamma_{s} / \gamma_{u, d}$ values. The third uncertainty in the branching fraction values represents the uncertainty from the value of $\gamma_{s} / \gamma_{u, d}$.

choose other reasonable values which depend on different assumptions. Three such choices and the resulting branching fraction values for the $B_{s}^{0} \rightarrow J / \psi \phi(1020)$ decay are listed in Table 6.5.

\subsection{Calculation of Ratios of Fragmentation Frac- tions}

In the previous section, the branching fractions were determined by taking $\gamma_{s} / \gamma_{u, d}=$ $0.40 \pm 0.06$ and assuming that $\gamma_{d}=\gamma_{u}$. Alternatively, one can calculate the ratios of the $\gamma$ values with the use of the matrix elements given in Equation 6.4 by making certain assumptions about the ratios of branching fractions. One can then use the same method that was described in Section 6.2, to make a weighted average of the matrix elements with common $\gamma$ values.

With the assumption that $\mathcal{B}\left(B^{+} \rightarrow J / \psi K^{+}\right)=\mathcal{B}\left(B^{0} \rightarrow J / \psi K^{0}\right)$ and $\mathcal{B}\left(B^{+} \rightarrow\right.$ $\left.J / \psi K^{*}(892)^{+}\right)=\mathcal{B}\left(B^{0} \rightarrow J / \psi K^{*}(892)^{0}\right)$ (which is reasonable considering that each 


\begin{tabular}{|l|l|}
\hline \hline$\gamma$ Relation & Value \\
\hline \hline$\gamma_{d} / \gamma_{u}$ & $(0.99 \pm 0.19$ (stat) $\pm 0.08($ syst $))$ \\
\hline$\gamma_{s} / \gamma_{u, d}$ & $(0.24 \pm 0.07$ (stat) $\pm 0.02($ syst $)) \cdot \frac{\mathcal{B}\left(B \rightarrow J / \psi K^{*}\right)}{\mathcal{B}(B s \rightarrow J / \psi \phi)}$ \\
\hline$\gamma_{s} / \gamma_{u, d}$ & $(0.39 \pm 0.11$ (stat) $\pm 0.04($ syst $)) \cdot \frac{\cdot(B(B \rightarrow J / \psi K)}{\mathcal{B}\left(B_{s} \rightarrow J / \psi \phi\right)}$ \\
\hline \hline
\end{tabular}

Table 6.6: Measured ratios of light quark production using various ratios of branching fractions.

pair of decays are similar $[38,39,40,41])$ one can measure the $\gamma_{d} / \gamma_{u}$ ratio by taking a weighted average of elements $(1,3)$ and $(2,4)$ or $(3,1)$ and $(4,2)$ given in Equation 6.4. The result of the weighted average for $\gamma_{d} / \gamma_{u}$ is shown in Table 6.6. It is of no surprise that the ratio is nearly 1 , since the masses of the up and down quarks are much smaller than the other quarks. Thus, the assumption that $\gamma_{u}=\gamma_{d}$ as was made in Section 6.2 seems reasonable. With this additional assumption, one can calculate the $\gamma_{s} / \gamma_{u, d}$ relationship by taking a weighted average of elements $[(1,5),(3,5)]$ or $[(2,5)$, $(4,5)]$. The results of the two additional weighted averages, which are functions of ratios of branching fractions, are also shown in Table 6.6.

Since all of the five decays are roughly similar, the branching fractions should be approximately equal. With this assumption, one can get a rough estimate for the ratio $\gamma_{s} / \gamma_{u, d}$ from Table 6.6. A better estimate would involve the use of the theoretical predictions for the ratio of the branching fractions. As can be seen from Figure 6.1, the predictions from References [38, 39] adequately describe the ratios of branching fractions that do not include the $B_{s}^{0}$ decay. Thus, it is reasonable to use the predictions for the ratio of branching fractions taken from References [38, 39] with the lifetimes scaled to the PDG values to calculate the ratio of strange quark to up or down quark production. The resulting ratio is shown in Table 6.7. As can be seen from the table, the ratio agrees with both the world average strangeness-suppression factor calculated in Reference [72] without the ALEPH [71] measurements as well as with the predicted values given in Equation 1.13. The measured value is also in agreement with the previous CDF strangeness-suppression factor [69].

As mentioned in Section 1.4, the measured ratio of $\gamma_{s} / \gamma_{u, d}$ should be smaller than the actual value due to the imbalance of the different flavor hadrons produced by decays from excited states. So the values in Table 6.7 should be viewed as a systematic underestimate of the actual ratio. Alternatively, one can consider the value to be the 


\begin{tabular}{|l|c|}
\hline \hline Method & Value for $\gamma_{s} / \gamma_{u, d}$ \\
\hline \hline Weighted Sum of 4 Ratios & $0.34 \pm 0.10$ (stat) \pm 0.03 (syst) \\
\hline \hline
\end{tabular}

Table 6.7: The ratio of strange quark to up or down quark production. The predictions for the ratios of branching fractions were taken from References [38, 39] with the lifetimes scaled to the PDG values. No uncertainties associated with the theoretical predictions were assigned.

ratio of the "observable" rate of flavored mesons, or $f_{s} / f_{u, d}$. Nevertheless, the value in Table 6.7 compares quite favorably with those in Table 6.2. Though the ratio $\gamma_{s} / \gamma_{u, d}$ might depend on the center-of-mass energy of the collisions that produced the light flavored quarks, it is hard to conclude this given the current uncertainties and the assumptions that are made. The general trend however does indicate a slight tendency for $\gamma_{s} / \gamma_{u, d}$ to increase slowly with center-of-mass energy [72].

As mentioned in Section 1.4, the fragmentation fractions for the various mesons are functions of the spectator quark masses. Thus, one can use the $\gamma$ ratios to estimate the mass differences between the various spectator quarks. Using the model described in Section 1.4 (with Equation 1.12) and the calculated value of $\gamma_{d} / \gamma_{u}=0.99 \pm$ 0.19 (stat) \pm 0.08 (syst), the squared mass difference between a $d$ quark and a $u$ quark is calculated to be $\left(6.4 \times 10^{-4} \pm 1.3 \times 10^{-2}\right)\left(\mathrm{GeV} / c^{2}\right)^{2}$. Using the calculated value of $\gamma_{s} / \gamma_{u, d}=0.34 \pm 0.10$ (stat) \pm 0.03 (syst) results in a calculated squared mass difference between a $s$ quark and a $u$ quark or a $d$ quark of $\left(6.9 \times 10^{-2} \pm 2.0 \times 10^{-2}\right)\left(\mathrm{GeV} / c^{2}\right)^{2}$. Both of these values are consistent with the range of values given by the PDG [7] which are listed in Table 1.3. It should be noted that these values are only indicative of the real mass differences since the values are based only on a specific model.

Using the same technique one can also calculate the charm quark mass when $B_{c}^{+}$ decays are observed. As can be seen from the values given in Equation 1.13, the value of $f_{c}$ is very sensitive to the $c$ quark mass. A relatively small change in the $c$ quark mass from $1000 \mathrm{MeV}$ to $1600 \mathrm{MeV}$ causes a change in $f_{c}$ of ten orders of magnitude. Alternatively, if the charm quark mass is known, the technique that was used to calculate the ratio of fragmentation fractions can also be used to test the hadronization model discussed in Section 1.4 as well as other hadronization models. For heavy quark and heavy anti-quark states such as $B_{c}^{+}$mesons, the fragmentation fractions have also been calculated using perturbative $\mathrm{QCD}$, which predicts the value 


\begin{tabular}{|l|c|c|}
\hline \hline Exp. & Measurement & Value $\left(\times 10^{-3}\right)$ \\
\hline \hline DELPHI & $f(b \rightarrow b-$ baryon $) \cdot \mathcal{B}\left(b-\right.$ baryon $\left.\rightarrow \Lambda_{c} l \bar{\nu}_{l} X\right)$ & $11.8 \pm 2.6_{-2.1}^{+3.1}$ \\
\hline ALEPH & $f\left(b \rightarrow \Lambda_{b}^{0}\right) \cdot \mathcal{B}\left(\Lambda_{b}^{0} \rightarrow \Lambda_{c}^{+} l^{-} \bar{\nu}_{l} X\right)$ & $15.1 \pm 2.9 \pm 2.3$ \\
\hline OPAL & $f\left(b \rightarrow \Lambda_{b}^{0}\right) \cdot \mathcal{B}\left(\Lambda_{b}^{0} \rightarrow \Lambda l^{-} \bar{\nu}_{l} X\right)$ & $2.91 \pm 0.23 \pm 0.25$ \\
\hline \hline
\end{tabular}

Table 6.8: Production and decay rates for various decays involving $\Lambda_{b}^{0}$ baryons taken from References $[79,80,81]$. The first uncertainties are statistical and the second uncertainties are systematic.

of $f_{c}$ to be around $1.5 \times 10^{-3}[77,78]$. Similar calculations have also been done for $B_{s}^{0}$ mesons, though the strange quark might not be massive enough to be useful in perturbative QCD calculations [29].

\subsection{Estimate of Fragmentation Fractions}

Given the ratios of $f_{s} / f_{u}$ and $f_{s} / f_{d}$, one can calculate the individual diquark "popping" probabilities or hadron fragmentation fractions if the baryon fragmentation fraction is known. Although the fragmentation fraction for bottom baryons is still unknown, but one can make an approximation with the available $\Lambda_{b}^{0}$ data. Currently, only the DELPHI, ALEPH and OPAL collaborations have published $\Lambda_{b}^{0}$ production rates, all of which involve semileptonic decays. Table 6.8 summarizes these $\Lambda_{b}^{0}$ rates.

Given the production rates from Table 6.8, one can calculate the $\Lambda_{b}^{0}$ fragmentation fraction with the use of the branching fractions taken from Table 6.9. The theoretical branching fraction for the decay $\Lambda_{b}^{0} \rightarrow X_{c} l \nu$ is taken to be the central value of the quoted range $(10-13) \%$ given in Reference [82]. The quoted range is based on the assumption that the semileptonic branching fractions for the $\Lambda_{b}^{0}$ baryon decays are the same as the measured semileptonic branching fractions of the $B^{+}$and $B^{0}$ meson decays.

The calculated $\Lambda_{b}^{0}$ fragmentation fractions for the various LEP measurements are listed in Table 6.10. For the weighted average given in Table 6.10, the weight factor for each measurement was taken to be the inverse of the squared statistical and squared systematic uncertainty.

Using the condition that the fragmentation fractions should sum to unity and assuming that the probability for a bottom quark to hadronize into a $B_{c}^{+}$meson is 


\begin{tabular}{|l|c|c|}
\hline \hline Measurement & Decay & Value \\
\hline \hline PDG & $\Lambda_{c}^{+} \rightarrow \Lambda X$ & $(35 \pm 11) \%$ \\
\hline Ref. [82] & $\Lambda_{b}^{0} \rightarrow X_{c} l \nu$ & $(10-13) \%$ \\
\hline \hline
\end{tabular}

Table 6.9: Branching fractions that are used in the calculation of the $\Lambda_{b}^{0}$ fragmentation fraction.

\begin{tabular}{|l|c|}
\hline \hline Method & $\Lambda_{b}^{0}$ production probability \\
\hline \hline OPAL & $(7.2 \pm 0.6 \pm 2.4) \%$ \\
\hline DELPHI & $\left(10.3 \pm 2.3_{-1.8}^{+2.7}\right) \%$ \\
\hline ALEPH & $(13.1 \pm 2.5 \pm 2.0) \%$ \\
\hline LEP Average & $(9.6 \pm 1.7) \%$ \\
\hline
\end{tabular}

Table 6.10: Probability to produce $\Lambda_{b}^{0}$ baryons using the LEP semileptonic $\Lambda_{b}^{0}$ decay rates. The calculated values do not include any uncertainties on the theoretical branching fractions.

very small $\left(f_{c} \ll 1\right)$, one obtains the relationship

$$
f_{u}+f_{d}+f_{s}+f_{\Lambda_{b}^{0}}=1 .
$$

The assumption that $f_{c} \ll 1$ is reasonable considering the values given in Equation 1.13. In addition, preliminary results from the CDF collaboration indicate that $f_{c}$ is at least one order of magnitude smaller than $f_{u}$ [83].

Equation 6.13 can then be rearranged to determine the values for $f_{u}, f_{d}$ and $f_{s}$ using the assumed value of $f_{\Lambda_{b}^{0}}$ and the measured ratios of fragmentation fractions. This gives the relationships

$$
\begin{aligned}
& f_{u}=\frac{1-f_{\Lambda_{b}^{\circ}}}{1+\frac{f_{d}+f_{s}}{f_{u}}}, \\
& f_{d}=\frac{1-f_{\Lambda_{b}^{\circ}}}{1+\frac{f_{u}+f_{s}}{f_{d}}}, \\
& f_{s}=\frac{1-f_{\Lambda_{b}^{\circ}}}{1+\frac{f_{u}+f_{d}}{f_{s}}} .
\end{aligned}
$$




\begin{tabular}{|l|c|c|}
\hline \hline Hadron/Method & LEP Average $f_{\Lambda_{b}^{0}}$ & UA1 $f_{\Lambda_{b}^{0}}$ \\
\hline \hline$B^{+} / B^{0}$ & $0.39 \pm 0.02 \pm 0.01$ & $0.37 \pm 0.02 \pm 0.01$ \\
\hline$B_{s}^{0}$ & $0.13 \pm 0.03 \pm 0.01$ & $0.13 \pm 0.03 \pm 0.01$ \\
\hline Baryon & $0.096 \pm 0.017$ & $0.14 \pm 0.02$ \\
\hline \hline
\end{tabular}

Table 6.11: Probabilities to produce the various bottom hadrons species with $f_{u}=f_{d}$ $=\gamma_{u}=\gamma_{d}$. The first column is based on the central value of the theoretical branching fraction for the decay $\mathcal{B}\left(\Lambda_{b}^{0} \rightarrow X_{c} l \nu\right)$ taken from Reference [82]. The second column is based on the assumption that the ratio of meson to baryon production measured at UA1 [72] is the same as the ratio of bottom meson to bottom baryon production at CDF. It should be noted that it was assumed that $f_{u}=f_{d}=\gamma_{u}=\gamma_{d}$ and the branching fractions for the bottom mesons were taken from References $[38,39]$ with the $B$ lifetimes scaled to the PDG values. The calculated values do not include any uncertainties on the theoretical branching fractions.

The resulting fragmentation fractions using these relationships with the assumption that $f_{u}=f_{d}$ are summarized in Table 6.11.

It should be noted that the calculated fragmentation fractions are based on the assumption that the branching fraction for the decay $\mathcal{B}\left(\Lambda_{b}^{0} \rightarrow X_{c} l \nu\right)$ is $11.5 \%$. As a check on the validity of this assumption, one can estimate the fragmentation fractions of the various bottom hadrons in another way. One can use a recent measurement of the meson to baryon production ratio at the CERN $S p \bar{p} S$ collider of $6.4 \pm 1.1$ to obtain a baryon fragmentation fraction of $13.5 \%$ [72]. One then assumes that the ratio of meson to baryon production measured at the $S p \bar{p} S$ collider is the same as the ratio of bottom meson to bottom baryon production at the Tevatron collider. The resulting calculation of the bottom hadron fragmentation fractions based on this assumption is also shown in Table 6.11.

It should also be noted that the probabilities given in Table 6.11 are also based on the assumption that $f_{u}=f_{d}$ or $\gamma_{u}=\gamma_{d}$. By removing this assumption, both the statistical and systematic uncertainties become larger. This is in part due to the fact that the uncertainties on the fragmentation fraction values for the $B^{+}$and $B^{0}$ mesons are correlated. The individual fragmentation fractions or production probabilities without this assumption are given in Table 6.12.

As can be seen from Tables 6.11 and 6.12 , both methods of estimating $f_{\Lambda_{b}^{\circ}}$ led to the same results within the given uncertainties. The first method, using the LEP data, 


\begin{tabular}{|l|c|c|}
\hline \hline Hadron/Method & LEP Average $f_{\Lambda_{b}^{0}}$ & UA1 $f_{\text {baryon }}$ \\
\hline \hline$B^{+}$ & $0.39 \pm 0.04 \pm 0.04$ & $0.37 \pm 0.03 \pm 0.04$ \\
\hline$B^{0}$ & $0.38 \pm 0.04 \pm 0.04$ & $0.37 \pm 0.03 \pm 0.04$ \\
\hline$B_{s}^{0}$ & $0.13 \pm 0.03 \pm 0.01$ & $0.13 \pm 0.03 \pm 0.01$ \\
\hline Baryon & $0.096 \pm 0.017$ & $0.14 \pm 0.02$ \\
\hline \hline
\end{tabular}

Table 6.12: Probabilities to produce the various bottom hadrons species. The first column is based on the central value of the theoretical branching fraction for the decay $\mathcal{B}\left(\Lambda_{b}^{0} \rightarrow X_{c} l \nu\right)$ taken from Reference [82]. The second column is based on the assumption that the ratio of meson to baryon production measured at UA1 [72] is the same as the ratio of bottom meson to bottom baryon production at CDF. It should be noted that the branching fractions for the bottom mesons were taken from References $[38,39]$ with the $B$ lifetimes scaled to the PDG values. The calculated values do not include any uncertainties on the theoretical branching fractions.

is preferred in that the calculation includes measured $\Lambda_{b}^{0}$ production times decay rates. The disadvantage of the first method is that it is based on the unmeasured branching fraction for the $\Lambda_{b}^{0} \rightarrow X_{c} l \nu$ decay. For example, as mentioned in Reference [81] the branching fraction for the decay $\Lambda_{b}^{0} \rightarrow X_{c} l \nu$ can be as low as $2.45 \%$. Nevertheless, it is interesting to note that the measured production probabilities are not far from the values given in Equation 1.13 or the values of $0.375,0.375,0.15$ and 0.1 which have generally been taken for the production probabilities for the $B^{+}, B^{0}, B_{s}^{0}$ and bottom baryons, respectively [84]. 


\section{Chapter 7}

\section{Summary of Results and Conclusions}

The Tevatron Collider at Fermilab was used to produce bottom mesons that were subsequently observed though five decay modes with the use of the Collider Detector at Fermilab. The five reconstructed decays (including the charge conjugate decays) are

- $B^{+} \rightarrow J / \psi K^{+}$

- $B^{+} \rightarrow J / \psi K^{*}(892)^{+}$

- $B^{0} \rightarrow J / \psi K^{0}$

- $B^{0} \rightarrow J / \psi K^{*}(892)^{0}$

- $B_{s}^{0} \rightarrow J / \psi \phi(1020)$

The daughter particles were reconstructed through the decay modes

- $J / \psi \rightarrow \mu^{+} \mu^{-}$

- $K^{*}(892)^{+} \rightarrow K^{0} \pi^{+}$

- $K^{0} \rightarrow K_{s}^{0} \rightarrow \pi^{+} \pi^{-}$

- $K^{*}(892)^{0} \rightarrow K^{+} \pi^{-}$

- $\phi(1020) \rightarrow K^{+} K^{-}$ 
Clear signals were visible in all of the five exclusive bottom meson decays as well as the five inclusive decays.

The ratios of branching fractions for the five bottom meson decays times their respective fragmentation fractions were determined by measuring the ratios of observed events divided by their respective detection efficiencies. These results were presented as a five-by-five matrix with each matrix element representing a ratio of any two of the five bottom meson decay rates listed above.

\subsection{Measurements of Branching Fractions}

Ratios of branching fractions for the five bottom meson decays of interest were then determined with the assumption that the probability to produce $B^{+}$mesons $\left(f_{u}\right)$ from a $\bar{p} p$ collisions at $\sqrt{s}=1.8 \mathrm{TeV}$ is the same as the probability to produce a $B^{0}$ mesons $\left(f_{d}\right)$. Given this assumption, the ratios of branching fractions were determined to be

- $\frac{\mathcal{B}\left(B^{+} \rightarrow J / \psi K^{*}(892)^{+}\right)}{\mathcal{B}\left(B^{+} \rightarrow J / \psi K^{+}\right)}=1.92 \pm 0.60 \pm 0.17$

- $\frac{\mathcal{B}\left(B^{0} \rightarrow J / \psi K^{0}\right)}{\mathcal{B}\left(B^{+} \rightarrow J / \psi K^{+}\right)}=1.15 \pm 0.27 \pm 0.10$

- $\frac{\mathcal{B}\left(B^{0} \rightarrow J / \psi K^{*}(892)^{0}\right)}{\mathcal{B}\left(B^{+} \rightarrow J / \psi K^{+}\right)}=1.59 \pm 0.33 \pm 0.12$,

- $\frac{\mathcal{B}\left(B_{s}^{0} \rightarrow J / \psi \phi(1020)\right)}{\mathcal{B}\left(B^{+} \rightarrow J / \psi K^{+}\right)}=0.41 \pm 0.12 \pm 0.04 \times \frac{f_{u}}{f_{s}}$,

- $\frac{\mathcal{B}\left(B^{0} \rightarrow J / \psi K^{0}\right)}{\mathcal{B}\left(B^{+} \rightarrow J / \psi K^{*}(892)^{+}\right)}=0.60 \pm 0.21 \pm 0.04$

- $\frac{\mathcal{B}\left(B^{0} \rightarrow J / \psi K^{*}(892)^{0}\right)}{\mathcal{B}\left(B^{+} \rightarrow J / \psi K^{*}(892)^{+}\right)}=0.83 \pm 0.27 \pm 0.07$

- $\frac{\mathcal{B}\left(B_{s}^{0} \rightarrow J / \psi \phi(1020)\right)}{\mathcal{B}\left(B^{+} \rightarrow J / \psi K^{*}(892)^{+}\right)}=0.21 \pm 0.08 \pm 0.02 \times \frac{f_{u}}{f_{s}}$,

- $\frac{\mathcal{B}\left(B^{0} \rightarrow J / \psi K^{*}(892)^{0}\right)}{\mathcal{B}\left(B^{0} \rightarrow J / \psi K^{0}\right)}=1.39 \pm 0.36 \pm 0.10$

- $\frac{\mathcal{B}\left(B_{s}^{0} \rightarrow J / \psi \phi(1020)\right)}{\mathcal{B}\left(B^{0} \rightarrow J / \psi K^{0}\right)}=0.35 \pm 0.12 \pm 0.03 \times \frac{f_{d}}{f_{s}}$,

- $\frac{\mathcal{B}\left(B_{s}^{0} \rightarrow J / \psi \phi(\mathbf{1 0 2 0})\right)}{\mathcal{B}\left(B^{0} \rightarrow J / \psi K^{*}(892)^{0}\right)}=0.26 \pm 0.08 \pm 0.02 \times \frac{f_{d}}{f_{s}}$

where the first uncertainties are statistical and the second are systematic. The observed ratios of branching fractions were found to be in good agreement with a phenomenological model based on a factorization ansatz of the quark currents. The good 
agreement also supported the assumptions that were used in the determination of the form factors which included chiral symmetry, heavy quark symmetries, simple pole dominance of the form factors and the normalization to semileptonic $D$ meson data.

The above ratios can also be used to improve the world average branching fractions for the decays in the numerator by multiplying the ratio of branching fractions with the current world average values for the branching fractions in the denominator. This leads to ten measurements which can be combined through a weighted average to improve their precision. The resulting weighted averages for the branching fractions of the five decays are

- $B^{+} \rightarrow J / \psi K^{+}=(0.82 \pm 0.18 \pm 0.07) \times 10^{-3}$,

- $B^{+} \rightarrow J / \psi K^{*}(892)^{+}=(1.73 \pm 0.55 \pm 0.15) \times 10^{-3}$,

- $B^{0} \rightarrow J / \psi K^{0}=(1.14 \pm 0.27 \pm 0.09) \times 10^{-3}$,

- $B^{0} \rightarrow J / \psi K^{*}(892)^{0}=(1.39 \pm 0.32 \pm 0.11) \times 10^{-3}$,

- $B_{s}^{0} \rightarrow J / \psi \phi(1020)=(0.37 \pm 0.11 \pm 0.04) \times 10^{-3} \times f_{u, d} / f_{s}$,

where the first uncertainties are statistical and the second are systematic.

The first measurement of the branching fraction for the decay $B_{s}^{0} \rightarrow J / \psi \phi(1020)$ is then determined by taking $f_{s} / f_{u, d}$ to be $0.40 \pm 0.06$. This results in a branching fraction of $(0.93 \pm 0.28 \pm 0.10 \pm 0.14) \times 10^{-3}$ where the first uncertainty is statistical, the second is systematic and the third is due to the uncertainty on the ratio $f_{u, d} / f_{s}$. Though the value of $f_{s} / f_{u, d}$ was taken to be $0.40 \pm 0.06$, one could have also used other values as was discussed in Section 6.2.

\subsection{Ratios of Fragmentation Fractions}

The ratios of branching fractions have also been used to determine the ratios of fragmentation fractions. Since the vector-pseudoscalar decays of the $B^{+}$and $B^{0}$ mesons and the vector-vector decays of the $B^{+}$and $B^{0}$ mesons are expected to have similar branching fractions, a weighted average of the ratio of both types of decays is performed to obtain the ratio of fragmentation fractions for the $B^{+}$and $B^{0}$ mesons. This ratio is determined to be $0.99 \pm 0.19$ (stat) \pm 0.08 (syst). With the additional 
assumption that the two fragmentation fractions are equal, the ratio of fragmentation fractions of the $B_{s}^{0}$ meson and either the $B^{+}$or $B^{0}$ meson have been calculated to be

$$
\frac{f_{s}}{f_{u}, f_{d}}=(0.24 \pm 0.07 \pm 0.02) \cdot \frac{\mathcal{B}\left(B \rightarrow J / \psi K^{*}\right)}{\mathcal{B}\left(B_{s} \rightarrow J / \psi \phi\right)}
$$

and

$$
\frac{f_{s}}{f_{u}, f_{d}}=(0.39 \pm 0.11 \pm 0.04) \cdot \frac{\mathcal{B}(B \rightarrow J / \psi K)}{\mathcal{B}\left(B_{s} \rightarrow J / \psi \phi\right)}
$$

where the first uncertainties are statistical and the second are systematic.

Using theoretical predictions based on a phenomenological model for the ratios of branching fractions, a weighted average of all the decays results in a ratio of fragmentation fractions for the $B_{s}^{0}$ meson and either the $B^{+}$or $B^{0}$ meson of 0.34 \pm 0.10 (stat) \pm 0.03 (syst). It should be noted that this ratio is similar to the rate of $\bar{s} s$ production relative to $\bar{u} u$ and $\bar{d} d$ production as measured in $e^{+} e^{-}$and deep inelastic scattering experiments. This implies that the hadronization mechanism is approximately flavor and energy independent.

The calculated fragmentation ratios also imply that the $u$ and $d$ quarks have a relatively similar mass as compared to the other quarks and that the squared mass difference between a $s$ quark and either a $u$ quark or a $d$ quark is $(6.9 \pm 2.0) \times$ $10^{-2}\left(\mathrm{GeV} / c^{2}\right)^{2}$. This value and the near equality of the $d$ and $u$ quark masses are consistent with the range of values given by the PDG [7].

Using the condition that the fragmentation fractions should sum to unity and assuming that the fragmentation fraction for charmed bottom mesons is negligible, the individual fragmentation fractions for the $B^{+}, B^{0}$ and $B_{s}^{0}$ mesons are determined as a function of the probability that a bottom quark hadronizes into a baryon. Based on the recent LEP semileptonic $\Lambda_{b}^{0}$ decay data, this probability has been estimated to be $0.096 \pm 0.017$. With this estimate, the fragmentation fractions for the $B^{+}, B^{0}$ and $B_{s}^{0}$ have been determined to be $0.39 \pm 0.04$ (stat) \pm 0.04 (syst), $0.38 \pm 0.04$ (stat) \pm 0.04 (syst) and $0.13 \pm 0.03$ (stat) \pm 0.01 (syst), respectively. These values are consistent with the assumed values that have been used in the past by various groups including the Particle Data Group. 


\subsection{Future Prospects}

The measurements of the ratios of branching fractions, individual branching fractions and fragmentation fractions have been limited by the number of reconstructed events. A data sample approximately four times larger than the one used for this analysis is currently being studied. The increased data set will result in smaller statistical uncertainties on the measured quantities. Additional data also allows the possibility to observe non-resonant decays and the opportunity for one to measure their branching fractions.

Using the techniques presented in this analysis, the larger data set can also be used to calculate ratios of branching fractions, individual branching fractions and fragmentation fractions with the use of semileptonic bottom hadron decays as well as hadronic decays involving $\psi(2 S)$ mesons instead of $J / \psi$ mesons. The five-by-five matrix can be extended to include the $\Lambda_{b}^{0} \rightarrow J / \psi \Lambda^{0}$ decay, thus eliminating the need to use LEP data to approximate $f_{\Lambda_{b}^{0}}$.

The technique that was used to calculate fragmentation fractions for the $B^{+}, B^{0}$ and $B_{s}^{0}$ mesons can also be used to determine the fragmentation fraction of $B_{c}^{+}$mesons when they are discovered and thus further test the current hadronization models. 


\section{Appendix A}

\section{Effect of Various Vertex Considerations}

In order to select the best vertexing and/or pointing requirements for the five decays of interest, four variables were examined with various transverse momentum requirements on the bottom and strange mesons. The four variables examined are: number of fitted bottom mesons decays, standard deviations of the mass resonances, signal to noise and significance of the resonance. These four variables were also examined using different primary $\bar{p} p$ beam position determination techniques. The various values are given in the tables below. 


\begin{tabular}{|c|c|c|c|c|c|}
\hline \hline Decay & No Pointing & 2-D Pointing & 3-D Pointing & 3-D + 2-D & 3-D + 3-D \\
\hline \hline$K^{+}$ & $182.1 \pm 23.8$ & $201.6 \pm 23.8$ & $200.1 \pm 22.9$ & - & \\
\hline$K^{*}(892)^{+}$ & $17.2 \pm 6.7$ & $9.7 \pm 6.0$ & $14.8 \pm 6.2$ & $14.9 \pm 5.5$ & $14.2 \pm 5.5$ \\
\hline$K^{0}$ & $42.3 \pm 9.4$ & $38.8 \pm 9.3$ & $40.5 \pm 8.6$ & $43.2 \pm 8.3$ & $40.0 \pm 8.2$ \\
\hline$K^{*}(892)^{0}$ & $121.5 \pm 25.3$ & $120.2 \pm 25.8$ & $130.7 \pm 26.2$ & - & - \\
\hline$\phi(1020)$ & $39.5 \pm 8.6$ & $30.5 \pm 8.0$ & $28.3 \pm 7.5$ & - & - \\
\hline \hline
\end{tabular}

Table A.1: Number of candidate $B$ meson events using store-averaged beam spot information with $P_{T}^{K}>1.5 \mathrm{GeV} / c$ and $P_{T}^{B}>6.0 \mathrm{GeV} / c$.

\begin{tabular}{|c|c|c|c|c|c|}
\hline \hline Decay & No Pointing & 2-D Pointing & 3-D Pointing & 3-D + 2-D & 3-D + 3-D \\
\hline \hline$K^{+}$ & $128.5 \pm 18.4$ & $142.5 \pm 18.0$ & $140.7 \pm 17.7$ & - & - \\
\hline$K^{*}(892)^{+}$ & $15.6 \pm 7.2$ & $14.1 \pm 5.6$ & $16.7 \pm 5.1$ & $15.0 \pm 4.7$ & $14.7 \pm 4.7$ \\
\hline$K^{0}$ & $29.3 \pm 7.6$ & $41.2 \pm 10.8$ & $29.7 \pm 6.8$ & $32.2 \pm 6.9$ & $28.0 \pm 6.6$ \\
\hline$K^{*}(892)^{0}$ & $115.1 \pm 19.5$ & $117.9 \pm 20.3$ & $112.6 \pm 19.6$ & - & - \\
\hline$\phi(1020)$ & $31.2 \pm 6.7$ & $26.4 \pm 6.1$ & $27.1 \pm 6.0$ & - & - \\
\hline \hline
\end{tabular}

Table A.2: Number of candidate $B$ meson events using store-averaged beam spot information with $P_{T}^{K}>2.0 \mathrm{GeV} / c$ and $P_{T}^{B}>6.0 \mathrm{GeV} / c$.

\begin{tabular}{|c|c|c|c|c|c|}
\hline \hline Decay & No Pointing & 2-D Pointing & 3-D Pointing & 3-D + 2-D & 3-D + 3-D \\
\hline \hline$K^{+}$ & $108.7 \pm 15.4$ & $123.6 \pm 15.5$ & $121.3 \pm 15.2$ & - & - \\
\hline$K^{*}(892)^{+}$ & $14.2 \pm 5.1$ & $13.7 \pm 5.2$ & $14.6 \pm 4.7$ & $12.7 \pm 4.3$ & $12.5 \pm 4.2$ \\
\hline$K^{0}$ & $28.3 \pm 7.1$ & $39.1 \pm 9.2$ & $28.2 \pm 6.2$ & $28.9 \pm 6.3$ & $25.9 \pm 6.0$ \\
\hline$K^{*}(892)^{0}$ & $112.0 \pm 15.8$ & $110.5 \pm 15.5$ & $103.8 \pm 15.1$ & - & - \\
\hline$\phi(1020)$ & $23.7 \pm 5.7$ & $21.1 \pm 5.4$ & $21.4 \pm 5.3$ & - & - \\
\hline \hline
\end{tabular}

Table A.3: Number of candidate $B$ meson events using store-averaged beam spot information with $P_{T}^{K}>2.0 \mathrm{GeV} / c$ and $P_{T}^{B}>8.0 \mathrm{GeV} / c$. 


\begin{tabular}{|c|c|c|c|c|c|}
\hline \hline Decay & No Pointing & 2-D Pointing & 3-D Pointing & 3-D + 2-D & 3-D + 3-D \\
\hline \hline$K^{+}$ & $200.0 \pm 24.7$ & N/A & $190.6 \pm 22.5$ & - & - \\
\hline$K^{*}(892)^{+}$ & $17.2 \pm 6.9$ & N/A & $12.9 \pm 5.3$ & $0.0 \pm 0.0$ & $13.0 \pm 5.3$ \\
\hline$K^{0}$ & $45.9 \pm 9.9$ & N/A & $41.4 \pm 8.4$ & $0.0 \pm 0.0$ & $40.6 \pm 8.1$ \\
\hline$K^{*}(892)^{0}$ & $123.1 \pm 23.3$ & N/A & $116.1 \pm 25.4$ & - & - \\
\hline$\phi(1020)$ & $38.3 \pm 8.6$ & N/A & $36.1 \pm 8.1$ & - & - \\
\hline \hline
\end{tabular}

Table A.4: Number of candidate $B$ meson events using event dependent beam spot information with $P_{T}^{K}>1.5 \mathrm{GeV} / c$ and $P_{T}^{B}>6.0 \mathrm{GeV} / c$.

\begin{tabular}{|c|c|c|c|c|c|}
\hline \hline Decay & No Pointing & 2-D Pointing & 3-D Pointing & 3-D + 2-D & 3-D + 3-D \\
\hline \hline$K^{+}$ & $133.5 \pm 18.8$ & N/A & $127.1 \pm 17.1$ & - & - \\
\hline$K^{*}(892)^{+}$ & $15.0 \pm 6.3$ & N/A & $16.4 \pm 5.1$ & $0.0 \pm 0.0$ & $12.1 \pm 4.4$ \\
\hline$K^{0}$ & $33.3 \pm 8.1$ & N/A & $31.7 \pm 6.8$ & $0.0 \pm 0.0$ & $30.4 \pm 6.8$ \\
\hline$K^{*}(892)^{0}$ & $113.5 \pm 19.5$ & N/A & $123.2 \pm 22.8$ & - & - \\
\hline$\phi(1020)$ & $29.5 \pm 6.5$ & N/A & $32.2 \pm 6.5$ & - & - \\
\hline \hline
\end{tabular}

Table A.5: Number of candidate $B$ meson events using event dependent beam spot information with $P_{T}^{K}>2.0 \mathrm{GeV} / c$ and $P_{T}^{B}>6.0 \mathrm{GeV} / c$.

\begin{tabular}{|c|c|c|c|c|c|}
\hline \hline Decay & No Pointing & 2-D Pointing & 3-D Pointing & 3-D + 2-D & 3-D + 3-D \\
\hline \hline$K^{+}$ & $112.2 \pm 16.4$ & N/A & $116.6 \pm 15.6$ & - & - \\
\hline$K^{*}(892)^{+}$ & $13.2 \pm 5.1$ & N/A & $14.3 \pm 4.7$ & $0.0 \pm 0.0$ & $9.9 \pm 4.0$ \\
\hline$K^{0}$ & $29.9 \pm 7.2$ & N/A & $29.4 \pm 6.3$ & $0.0 \pm 0.0$ & $28.3 \pm 6.3$ \\
\hline$K^{*}(892)^{0}$ & $107.0 \pm 15.2$ & N/A & $103.8 \pm 15.2$ & - & - \\
\hline$\phi(1020)$ & $24.3 \pm 5.8$ & N/A & $28.9 \pm 6.0$ & - & - \\
\hline \hline
\end{tabular}

Table A.6: Number of candidate $B$ meson events using event dependent beam spot information with $P_{T}^{K}>2.0 \mathrm{GeV} / c$ and $P_{T}^{B}>8.0 \mathrm{GeV} / c$. 


\begin{tabular}{|c|c|c|c|c|c|}
\hline \hline Decay & No Pointing & 2-D Pointing & 3-D Pointing & 3-D + 2-D & 3-D + 3-D \\
\hline \hline$K^{+}$ & $13.3 \pm 1.8$ & $13.7 \pm 1.7$ & $13.0 \pm 1.5$ & - & - \\
\hline$K^{*}(892)^{+}$ & $10.4 \pm 4.4$ & $12.4 \pm 5.4$ & $9.0 \pm 3.7$ & $8.3 \pm 2.6$ & $8.2 \pm 2.7$ \\
\hline$K^{0}$ & $12.9 \pm 2.6$ & $12.1 \pm 3.4$ & $10.3 \pm 2.4$ & $9.9 \pm 1.7$ & $10.7 \pm 2.0$ \\
\hline$K^{*}(892)^{0}$ & $8.5 \pm 2.3$ & $10.2 \pm 2.8$ & $11.5 \pm 2.7$ & - & - \\
\hline$\phi(1020)$ & $12.3 \pm 2.6$ & $13.0 \pm 3.0$ & $11.5 \pm 2.6$ & - & - \\
\hline \hline
\end{tabular}

Table A.7: Gaussian width of candidate $B$ meson signal region using store-averaged beam spot information with $P_{T}^{K}>1.5 \mathrm{GeV} / c$ and $P_{T}^{B}>6.0 \mathrm{GeV} / c$.

\begin{tabular}{|c|c|c|c|c|c|}
\hline \hline Decay & No Pointing & 2-D Pointing & 3-D Pointing & 3-D + 2-D & 3-D + 3-D \\
\hline \hline$K^{+}$ & $13.8 \pm 2.2$ & $13.3 \pm 1.8$ & $13.0 \pm 1.7$ & - & - \\
\hline$K^{*}(892)^{+}$ & $9.5 \pm 7.3$ & $11.3 \pm 5.2$ & $10.8 \pm 2.5$ & $7.7 \pm 2.1$ & $7.6 \pm 2.0$ \\
\hline$K^{0}$ & $13.9 \pm 3.2$ & $32.7 \pm 8.1$ & $9.2 \pm 2.2$ & $9.2 \pm 1.9$ & $9.7 \pm 2.2$ \\
\hline$K^{*}(892)^{0}$ & $12.8 \pm 2.3$ & $13.5 \pm 2.6$ & $13.8 \pm 2.6$ & - & - \\
\hline$\phi(1020)$ & $13.4 \pm 2.2$ & $12.4 \pm 2.1$ & $11.3 \pm 1.8$ & - & - \\
\hline \hline
\end{tabular}

Table A.8: Gaussian width of candidate $B$ meson signal region using store-averaged beam spot information with $P_{T}^{K}>2.0 \mathrm{GeV} / c$ and $P_{T}^{B}>6.0 \mathrm{GeV} / c$.

\begin{tabular}{|c|c|c|c|c|c|}
\hline \hline Decay & No Pointing & 2-D Pointing & 3-D Pointing & 3-D + 2-D & 3-D + 3-D \\
\hline \hline$K^{+}$ & $14.1 \pm 2.2$ & $13.9 \pm 1.8$ & $13.4 \pm 1.8$ & - & - \\
\hline$K^{*}(892)^{+}$ & $11.1 \pm 3.3$ & $12.4 \pm 4.9$ & $10.8 \pm 2.7$ & $7.2 \pm 2.0$ & $7.1 \pm 2.0$ \\
\hline$K^{0}$ & $14.6 \pm 3.6$ & $31.3 \pm 6.4$ & $8.9 \pm 2.1$ & $8.6 \pm 1.9$ & $8.8 \pm 2.1$ \\
\hline$K^{*}(892)^{0}$ & $11.5 \pm 1.7$ & $11.4 \pm 1.8$ & $11.5 \pm 1.8$ & - & - \\
\hline$\phi(1020)$ & $13.1 \pm 2.5$ & $12.5 \pm 2.4$ & $11.1 \pm 2.2$ & - & - \\
\hline \hline
\end{tabular}

Table A.9: Gaussian width of candidate $B$ meson signal region using store-averaged beam spot information with $P_{T}^{K}>2.0 \mathrm{GeV} / c$ and $P_{T}^{B}>8.0 \mathrm{GeV} / c$. 


\begin{tabular}{|c|c|c|c|c|c|}
\hline \hline Decay & No Pointing & 2-D Pointing & 3-D Pointing & 3-D + 2-D & 3-D + 3-D \\
\hline \hline$K^{+}$ & $13.5 \pm 1.8$ & N/A & $12.5 \pm 1.5$ & - & - \\
\hline$K^{*}(892)^{+}$ & $11.2 \pm 3.6$ & N/A & $5.1 \pm 1.8$ & N/A & $7.0 \pm 3.1$ \\
\hline$K^{0}$ & $13.9 \pm 2.8$ & N/A & $9.8 \pm 1.9$ & N/A & $9.7 \pm 1.9$ \\
\hline$K^{*}(892)^{0}$ & $9.5 \pm 1.8$ & N/A & $10.5 \pm 2.8$ & - & - \\
\hline$\phi(1020)$ & $13.1 \pm 2.6$ & N/A & $13.1 \pm 2.6$ & - & - \\
\hline \hline
\end{tabular}

Table A.10: Gaussian width of candidate $B$ meson signal region using event dependent beam spot information with $P_{T}^{K}>1.5 \mathrm{GeV} / c$ and $P_{T}^{B}>6.0 \mathrm{GeV} / c$.

\begin{tabular}{|c|c|c|c|c|c|}
\hline \hline Decay & No Pointing & 2-D Pointing & 3-D Pointing & 3-D + 2-D & 3-D + 3-D \\
\hline \hline$K^{+}$ & $14.4 \pm 2.2$ & N/A & $13.2 \pm 1.8$ & - & - \\
\hline$K^{*}(892)^{+}$ & $10.9 \pm 5.9$ & N/A & $10.4 \pm 2.6$ & N/A & $8.9 \pm 2.6$ \\
\hline$K^{0}$ & $15.0 \pm 3.4$ & N/A & $9.0 \pm 1.9$ & N/A & $10.0 \pm 2.2$ \\
\hline$K^{*}(892)^{0}$ & $12.6 \pm 2.4$ & N/A & $15.4 \pm 3.5$ & - & - \\
\hline$\phi(1020)$ & $14.0 \pm 2.4$ & N/A & $12.2 \pm 2.0$ & - & - \\
\hline \hline
\end{tabular}

Table A.11: Gaussian width of candidate $B$ meson signal region using event dependent beam spot information with $P_{T}^{K}>2.0 \mathrm{GeV} / c$ and $P_{T}^{B}>6.0 \mathrm{GeV} / c$.

\begin{tabular}{|c|c|c|c|c|c|}
\hline \hline Decay & No Pointing & 2-D Pointing & 3-D Pointing & 3-D + 2-D & 3-D + 3-D \\
\hline \hline$K^{+}$ & $15.4 \pm 2.6$ & N/A & $14.4 \pm 2.2$ & - & - \\
\hline$K^{*}(892)^{+}$ & $11.8 \pm 4.0$ & N/A & $9.9 \pm 2.4$ & N/A & $8.7 \pm 3.0$ \\
\hline$K^{0}$ & $14.6 \pm 3.4$ & N/A & $8.7 \pm 1.9$ & N/A & $9.0 \pm 2.2$ \\
\hline$K^{*}(892)^{0}$ & $11.2 \pm 1.6$ & N/A & $11.3 \pm 1.9$ & - & - \\
\hline$\phi(1020)$ & $13.4 \pm 2.6$ & N/A & $12.2 \pm 2.2$ & - & - \\
\hline \hline
\end{tabular}

Table A.12: Gaussian width of candidate $B$ meson signal region using event dependent beam spot information with $P_{T}^{K}>2.0 \mathrm{GeV} / c$ and $P_{T}^{B}>8.0 \mathrm{GeV} / c$. 


\begin{tabular}{|c|c|c|c|c|c|}
\hline \hline Decay & No Pointing & 2-D Pointing & 3-D Pointing & 3-D + 2-D & 3-D + 3-D \\
\hline \hline$K^{+}$ & 0.633 & 0.758 & 0.777 & - & - \\
\hline$K^{*}(892)^{+}$ & 0.539 & 0.361 & 0.635 & 0.672 & 0.618 \\
\hline$K^{0}$ & 1.049 & 1.103 & 1.361 & 1.632 & 1.523 \\
\hline$K^{*}(892)^{0}$ & 0.188 & 0.210 & 0.237 & - & - \\
\hline$\phi(1020)$ & 1.255 & 1.102 & 1.036 & - & - \\
\hline \hline
\end{tabular}

Table A.13: Ratio of candidate $B$ meson signal to background using store-averaged beam spot information with $P_{T}^{K}>1.5 \mathrm{GeV} / c$ and $P_{T}^{B}>6.0 \mathrm{GeV} / c$.

\begin{tabular}{|c|c|c|c|c|c|}
\hline \hline Decay & No Pointing & 2-D Pointing & 3-D Pointing & 3-D + 2-D & 3-D + 3-D \\
\hline \hline$K^{+}$ & 0.920 & 1.090 & 1.117 & - & - \\
\hline$K^{*}(892)^{+}$ & 1.034 & 1.207 & 1.592 & 1.409 & 1.336 \\
\hline$K^{0}$ & 1.260 & 2.212 & 1.754 & 2.142 & 1.846 \\
\hline$K^{*}(892)^{0}$ & 0.440 & 0.467 & 0.464 & - & - \\
\hline$\phi(1020)$ & 2.918 & 2.699 & 2.737 & - & - \\
\hline \hline
\end{tabular}

Table A.14: Ratio of candidate $B$ meson signal to background using store-averaged beam spot information with $P_{T}^{K}>2.0 \mathrm{GeV} / c$ and $P_{T}^{B}>6.0 \mathrm{GeV} / c$.

\begin{tabular}{|c|c|c|c|c|c|}
\hline \hline Decay & No Pointing & 2-D Pointing & 3-D Pointing & 3-D + 2-D & 3-D + 3-D \\
\hline \hline$K^{+}$ & 1.282 & 1.583 & 1.607 & - & - \\
\hline$K^{*}(892)^{+}$ & 1.213 & 1.469 & 1.673 & 1.481 & 1.411 \\
\hline$K^{0}$ & 1.858 & 4.263 & 2.475 & 2.689 & 2.443 \\
\hline$K^{*}(892)^{0}$ & 0.711 & 0.735 & 0.715 & - & - \\
\hline$\phi(1020)$ & 3.261 & 3.038 & 3.039 & - & - \\
\hline \hline
\end{tabular}

Table A.15: Ratio of candidate $B$ meson signal to background using store-averaged beam spot information with $P_{T}^{K}>2.0 \mathrm{GeV} / c$ and $P_{T}^{B}>8.0 \mathrm{GeV} / c$. 


\begin{tabular}{|c|c|c|c|c|c|}
\hline \hline Decay & No Pointing & 2-D Pointing & 3-D Pointing & 3-D + 2-D & 3-D + 3-D \\
\hline \hline$K^{+}$ & 0.764 & N/A & 0.660 & - & - \\
\hline$K^{*}(892)^{+}$ & 0.531 & N/A & 0.539 & N/A & 0.626 \\
\hline$K^{0}$ & 1.137 & N/A & 1.397 & N/A & 1.544 \\
\hline$K^{*}(892)^{0}$ & 0.203 & N/A & 0.207 & - & - \\
\hline$\phi(1020)$ & 1.302 & N/A & 1.433 & - & - \\
\hline \hline
\end{tabular}

Table A.16: Ratio of candidate $B$ meson signal to background using event dependent beam spot information with $P_{T}^{K}>1.5 \mathrm{GeV} / c$ and $P_{T}^{B}>6.0 \mathrm{GeV} / c$.

\begin{tabular}{|c|c|c|c|c|c|}
\hline \hline Decay & No Pointing & 2-D Pointing & 3-D Pointing & 3-D + 2-D & 3-D + 3-D \\
\hline \hline$K^{+}$ & 1.054 & N/A & 0.910 & - & - \\
\hline$K^{*}(892)^{+}$ & 0.945 & N/A & 1.436 & N/A & 1.150 \\
\hline$K^{0}$ & 1.464 & N/A & 1.888 & N/A & 2.035 \\
\hline$K^{*}(892)^{0}$ & 0.433 & N/A & 0.511 & - & - \\
\hline$\phi(1020)$ & 2.844 & N/A & 3.664 & - & - \\
\hline \hline
\end{tabular}

Table A.17: Ratio of candidate $B$ meson signal to background using event dependent beam spot information with $P_{T}^{K}>2.0 \mathrm{GeV} / c$ and $P_{T}^{B}>6.0 \mathrm{GeV} / c$.

\begin{tabular}{|c|c|c|c|c|c|}
\hline \hline Decay & No Pointing & 2-D Pointing & 3-D Pointing & 3-D + 2-D & 3-D + 3-D \\
\hline \hline$K^{+}$ & 1.473 & N/A & 1.366 & - & - \\
\hline$K^{*}(892)^{+}$ & 1.072 & N/A & 1.570 & N/A & 1.189 \\
\hline$K^{0}$ & 2.022 & N/A & 2.673 & N/A & 2.820 \\
\hline$K^{*}(892)^{0}$ & 0.690 & N/A & 0.729 & - & - \\
\hline$\phi(1020)$ & 3.564 & N/A & 4.752 & - & - \\
\hline \hline
\end{tabular}

Table A.18: Ratio of candidate $B$ meson signal to background using event dependent beam spot information with $P_{T}^{K}>2.0 \mathrm{GeV} / c$ and $P_{T}^{B}>8.0 \mathrm{GeV} / c$. 


\begin{tabular}{|c|c|c|c|c|c|}
\hline \hline Decay & No Pointing & 2-D Pointing & 3-D Pointing & 3-D + 2-D & 3-D + 3-D \\
\hline \hline$K^{+}$ & 7.64 & 8.47 & 8.74 & - & - \\
\hline$K^{*}(892)^{+}$ & 2.57 & 1.62 & 2.41 & 2.68 & 2.58 \\
\hline$K^{0}$ & 4.48 & 4.18 & 4.71 & 5.23 & 4.89 \\
\hline$K^{*}(892)^{0}$ & 4.81 & 4.66 & 4.99 & - & - \\
\hline$\phi(1020)$ & 4.38 & 3.83 & 3.78 & - & - \\
\hline \hline
\end{tabular}

Table A.19: Significance of candidate $B$ meson signal (fitted number of events over uncertainty) using store-averaged beam spot information with $P_{T}^{K}>1.5 \mathrm{GeV} / c$ and $P_{T}^{B}>6.0 \mathrm{GeV} / c$.

\begin{tabular}{|c|c|c|c|c|c|}
\hline \hline Decay & No Pointing & 2-D Pointing & 3-D Pointing & 3-D + 2-D & 3-D + 3-D \\
\hline \hline$K^{+}$ & 7.00 & 7.92 & 7.96 & - & - \\
\hline$K^{*}(892)^{+}$ & 2.15 & 2.54 & 3.28 & 3.18 & 3.13 \\
\hline$K^{0}$ & 3.84 & 3.81 & 4.39 & 4.69 & 4.25 \\
\hline$K^{*}(892)^{\mathbf{0}}$ & 5.90 & 5.80 & 5.74 & - & - \\
\hline$\phi(1020)$ & 4.68 & 4.34 & 4.49 & - & - \\
\hline \hline
\end{tabular}

Table A.20: Significance of candidate $B$ meson signal (fitted number of events over uncertainty) using store-averaged beam spot information with $P_{T}^{K}>2.0 \mathrm{GeV} / c$ and $P_{T}^{B}>6.0 \mathrm{GeV} / c$.

\begin{tabular}{|c|c|c|c|c|c|}
\hline \hline Decay & No Pointing & 2-D Pointing & 3-D Pointing & 3-D + 2-D & 3-D + 3-D \\
\hline \hline$K^{+}$ & 7.04 & 7.97 & 7.98 & - & - \\
\hline$K^{*}(892)^{+}$ & 2.80 & 2.65 & 3.08 & 2.98 & 2.95 \\
\hline$K^{\mathbf{0}}$ & 3.99 & 4.26 & 4.52 & 4.60 & 4.31 \\
\hline$K^{*}(892)^{0}$ & 7.10 & 7.12 & 6.85 & - & - \\
\hline$\phi(1020)$ & 4.13 & 3.92 & 4.01 & - & - \\
\hline \hline
\end{tabular}

Table A.21: Significance of candidate $B$ meson signal (fitted number of events over uncertainty) using store-averaged beam spot information with $P_{T}^{K}>2.0 \mathrm{GeV} / c$ and $P_{T}^{B}>8.0 \mathrm{GeV} / c$. 


\begin{tabular}{|c|c|c|c|c|c|}
\hline \hline Decay & No Pointing & 2-D Pointing & 3-D Pointing & 3-D + 2-D & 3-D + 3-D \\
\hline \hline$K^{+}$ & 8.08 & N/A & 8.48 & - & - \\
\hline$K^{*}(892)^{+}$ & 2.50 & N/A & 2.44 & N/A & 2.44 \\
\hline$K^{0}$ & 4.63 & N/A & 4.96 & N/A & 5.00 \\
\hline$K^{*}(892)^{0}$ & 5.28 & N/A & 4.57 & - & - \\
\hline$\phi(1020)$ & 4.43 & N/A & 4.44 & - & - \\
\hline \hline
\end{tabular}

Table A.22: Significance of candidate $B$ meson signal (fitted number of events over uncertainty) using event dependent beam spot information with $P_{T}^{K}>1.5 \mathrm{GeV} / \mathrm{c}$ and $P_{T}^{B}>6.0 \mathrm{GeV} / c$.

\begin{tabular}{|c|c|c|c|c|c|}
\hline \hline Decay & No Pointing & 2-D Pointing & 3-D Pointing & 3-D + 2-D & 3-D + 3-D \\
\hline \hline$K^{+}$ & 7.10 & N/A & 7.43 & - & - \\
\hline$K^{*}(892)^{+}$ & 2.38 & N/A & 3.20 & N/A & 2.71 \\
\hline$K^{0}$ & 4.12 & N/A & 4.64 & N/A & 4.48 \\
\hline$K^{*}(892)^{0}$ & 5.81 & N/A & 5.74 & - & - \\
\hline$\phi(1020)$ & 4.52 & N/A & 4.98 & - & - \\
\hline \hline
\end{tabular}

Table A.23: Significance of candidate $B$ meson signal (fitted number of events over uncertainty) using event dependent beam spot information with $P_{T}^{K}>2.0 \mathrm{GeV} / c$ and $P_{T}^{B}>6.0 \mathrm{GeV} / c$.

\begin{tabular}{|c|c|c|c|c|c|}
\hline \hline Decay & No Pointing & 2-D Pointing & 3-D Pointing & 3-D + 2-D & 3-D + 3-D \\
\hline \hline$K^{+}$ & 6.84 & N/A & 7.46 & - & - \\
\hline$K^{*}(892)^{+}$ & 2.57 & N/A & 3.08 & N/A & 2.46 \\
\hline$K^{0}$ & 4.17 & N/A & 4.66 & N/A & 4.52 \\
\hline$K^{*}(892)^{0}$ & 7.03 & N/A & 6.86 & - & - \\
\hline$\phi(1020)$ & 4.22 & N/A & 4.80 & - & - \\
\hline \hline
\end{tabular}

Table A.24: Significance of candidate $B$ meson signal (fitted number of events over uncertainty) using event dependent beam spot information with $P_{T}^{K}>2.0 \mathrm{GeV} / c$ and $P_{T}^{B}>8.0 \mathrm{GeV} / c$. 


\section{Appendix B}

\section{Matrix Elements of Ratio of Events and Ratios of Detection Efficiencies}

The matrix elements given by the ratio of branching fractions times fragmentation fractions divided by the ratios of detection efficiencies can be calculated using the number of observed events as listed in Tables 3.8 and 3.9 for the five decays of interest using different and similar respective transverse momentum requirements on the bottom and strange mesons.

The ratio of detection efficiencies can be calculated with the individual efficiencies values given in Tables 4.3 through 4.10 . The uncertainties on the calculated efficiencies values are taken to be a source of systematic uncertainties. The addition sources of systematic uncertainties that are given in the tables below correspond to the values given in Chapter 5 .

The number of events and efficiencies that do not cancel in a given matrix element $(i, j)$ are summarized in the tables given below. The matrix element indices correspond to the following order of decays

1. $B^{+} \rightarrow J / \psi K^{+}$,

2. $B^{+} \rightarrow J / \psi K^{*}(892)^{+}$,

3. $B^{0} \rightarrow J / \psi K^{0}$,

4. $B^{0} \rightarrow J / \psi K^{*}(892)^{0}$,

5. $B_{s}^{0} \rightarrow J / \psi \phi(1020)$. 


\begin{tabular}{|l|c|}
\hline \hline Variable & Value \\
\hline \hline $\mathrm{N}\left(B^{+} \rightarrow J / \psi K^{+}\right)$ & $153.5 \pm 18.9$ \\
\hline $\mathrm{N}\left(B^{+} \rightarrow J / \psi K^{*}(892)^{+}\right)$ & $21.3 \pm 6.1$ \\
\hline$\epsilon_{M C}\left(B^{+} \rightarrow J / \psi K^{+}\right)$ & $0.663 \%$ \\
\hline$\epsilon_{M C}\left(B^{+} \rightarrow J / \psi K^{*}(892)^{+}\right)$ & $0.0619 \%$ \\
\hline$\epsilon_{\mathcal{~} \tau(B)}\left(B^{+} \rightarrow J / \psi K^{+}\right)$ & $(90.0 \pm 0.5) \%$ \\
\hline$\epsilon_{c \tau(B)}\left(B^{+} \rightarrow J / \psi K^{*}(892)^{+}\right)$ & $(88.0 \pm 0.6) \%$ \\
\hline$\epsilon_{c \tau\left(K_{s}^{0}\right)}$ & $(95.8 \pm 0.7) \%$ \\
\hline$\epsilon\left(C . L . K_{s}^{0}\right.$ vertex) & $(93.8 \pm 1.7) \%$ \\
\hline$\epsilon\left(C . L . K_{s}^{0}\right.$ mass and vertex) & $(98.3 \pm 0.6) \%$ \\
\hline$\epsilon\left(K_{s}^{0}\right.$ tracking) & $(89.5 \pm 1.0) \%$ \\
\hline Helicity (syst) & $\pm 2.5 \%$ \\
\hline Monte Carlo (syst) & $\pm 5 \%$ \\
\hline$C . L .(B$ vertex) (syst) & $\pm 1 \%$ \\
\hline Decay of Kaon (syst) & $\pm 3 \%$ \\
\hline$P_{T}$ spectrum (syst) & $\pm 4.6 \%$ \\
\hline Effect of Excited states $\left(B^{+} \rightarrow J / \psi K^{+}\right)($syst) & $\pm 2.7 \%$ \\
\hline Effect of Excited states $\left(B^{+} \rightarrow J / \psi K^{*}(892)^{+}\right)$(syst) & $\pm 2.0 \%$ \\
\hline \hline
\end{tabular}

Table B.1: Numbers that go into the calculation of the matrix elements $(1,2)$ and $(2,1)$ with different momentum cuts on the bottom and strange mesons. 


\begin{tabular}{|l|c|}
\hline \hline Variable & Value \\
\hline \hline $\mathrm{N}\left(B^{+} \rightarrow J / \psi K^{+}\right)$ & $123.3 \pm 15.4$ \\
\hline $\mathrm{N}\left(B^{+} \rightarrow J / \psi K^{*}(892)^{+}\right)$ & $20.0 \pm 5.9$ \\
\hline$\epsilon_{M C}\left(B^{+} \rightarrow J / \psi K^{+}\right)$ & $0.584 \%$ \\
\hline$\epsilon_{M C}\left(B^{+} \rightarrow J / \psi K^{*}(892)^{+}\right)$ & $0.0588 \%$ \\
\hline$\epsilon_{c \tau(B)}\left(B^{+} \rightarrow J / \psi K^{+}\right)$ & $(90.0 \pm 0.5) \%$ \\
\hline$\epsilon_{c \tau(B)}\left(B^{+} \rightarrow J / \psi K^{*}(892)^{+}\right)$ & $(88.0 \pm 0.6) \%$ \\
\hline$\epsilon_{c \tau}\left(K_{s}\right)$ & $(95.8 \pm 0.7) \%$ \\
\hline$\epsilon\left(C . L . K_{s}^{0}\right.$ vertex) & $(93.8 \pm 1.7) \%$ \\
\hline$\epsilon\left(C . L . K_{s}^{0}\right.$ mass and vertex) & $(98.3 \pm 0.6) \%$ \\
\hline$\epsilon\left(K_{s}^{0}\right.$ tracking) & $(89.5 \pm 1.0) \%$ \\
\hline Helicity (syst) & $\pm 2.5 \%$ \\
\hline Monte Carlo (syst) & $\pm 5 \%$ \\
\hline$C . L .(B$ vertex) (syst) & $\pm 1 \%$ \\
\hline Decay of Kaon (syst) & $\pm 3 \%$ \\
\hline$P_{T}$ spectrum & $\pm 4.1 \%$ \\
\hline Effect of Excites States $\left(B^{+} \rightarrow J / \psi K^{+}\right)$(syst) & $\pm 2.7 \%$ \\
\hline Effect of Excites States $\left(B^{+} \rightarrow J / \psi K^{*}(892)^{+}\right)$(syst) & $\pm 2.0 \%$ \\
\hline \hline
\end{tabular}

Table B.2: Numbers that go into the calculation of the matrix elements $(1,2)$ and $(2,1)$ with same momentum cuts on the bottom and strange mesons. 


\begin{tabular}{|l|c|}
\hline \hline Variable & Value \\
\hline \hline $\mathrm{N}\left(B^{+} \rightarrow J / \psi K^{+}\right)$ & $153.5 \pm 18.9$ \\
\hline $\mathrm{N}\left(B^{0} \rightarrow J / \psi K^{0}\right)$ & $36.9 \pm 7.3$ \\
\hline$\epsilon_{M C}\left(B^{+} \rightarrow J / \psi K^{+}\right)$ & $0.663 \%$ \\
\hline$\epsilon_{M C}\left(B^{0} \rightarrow J / \psi K^{0}\right)$ & $0.177 \%$ \\
\hline$\epsilon_{c r(B)}\left(B^{+} \rightarrow J / \psi K^{+}\right)$ & $(90.0 \pm 0.5) \%$ \\
\hline$\epsilon_{c r(B)}\left(B^{0} \rightarrow J / \psi K^{0}\right)$ & $(87.6 \pm 0.7) \%$ \\
\hline$\epsilon_{c r\left(K_{s}\right)}$ & $(95.8 \pm 0.7) \%$ \\
\hline$\epsilon\left(C . L . K_{s}^{0}\right.$ vertex) & $(93.8 \pm 1.7) \%$ \\
\hline$\epsilon\left(C . L . K_{s}^{0}\right.$ mass and vertex) & $(98.3 \pm 0.6) \%$ \\
\hline$\epsilon(K \pi$ tracking) & $(97.9 \pm 0.7) \%$ \\
\hline$\epsilon\left(K_{s}^{0}\right.$ tracking) & $(89.5 \pm 1.0) \%$ \\
\hline Monte Carlo (syst) & $\pm 5 \%$ \\
\hline$C . L .(B$ vertex) (syst) & $\pm 1 \%$ \\
\hline Decay of Kaon (syst) & $\pm 3 \%$ \\
\hline$P_{T}$ spectrum & $\pm 4.8 \%$ \\
\hline Effect of Excites States $\left(B^{+} \rightarrow J / \psi K^{+}\right)$(syst) & $\pm 2.7 \%$ \\
\hline Effect of Excites States $\left(B^{0} \rightarrow J / \psi K^{0}\right)$ (syst) & $\pm 1.1 \%$ \\
\hline \hline
\end{tabular}

Table B.3: Numbers that go into the calculation of the matrix elements $(1,3)$ and $(3,1)$ with different momentum cuts on the bottom and strange mesons. 


\begin{tabular}{|l|c|}
\hline \hline Variable & Value \\
\hline \hline $\mathrm{N}\left(B^{+} \rightarrow J / \psi K^{+}\right)$ & $123.3 \pm 15.4$ \\
\hline $\mathrm{N}\left(B^{0} \rightarrow J / \psi K^{0}\right)$ & $25.5 \pm 5.7$ \\
\hline$\epsilon_{M C}\left(B^{+} \rightarrow J / \psi K^{+}\right)$ & $0.584 \%$ \\
\hline$\epsilon_{M C}\left(B^{0} \rightarrow J / \psi K^{0}\right)$ & $0.136 \%$ \\
\hline$\epsilon_{c \tau(B)}\left(B^{+} \rightarrow J / \psi K^{+}\right)$ & $(90.0 \pm 0.5) \%$ \\
\hline$\epsilon_{c \tau(B)}\left(B^{0} \rightarrow J / \psi K^{0}\right)$ & $(87.6 \pm 0.7) \%$ \\
\hline$\epsilon_{c \tau(K s)}\left(K_{s}\right)$ vertex) & $(95.8 \pm 0.7) \%$ \\
\hline$\epsilon\left(C . L . K_{s}^{0}\right)$ & $(93.8 \pm 1.7) \%$ \\
\hline$\epsilon\left(C . L . K_{s}^{0}\right.$ mass and vertex) & $(98.3 \pm 0.6) \%$ \\
\hline$\epsilon(K \pi$ tracking) & $(97.9 \pm 0.7) \%$ \\
\hline$\epsilon\left(K_{s}^{0}\right.$ tracking) & $(89.5 \pm 1.0) \%$ \\
\hline Monte Carlo (syst) & $\pm 5 \%$ \\
\hline$C . L .(B$ vertex) (syst) & $\pm 1 \%$ \\
\hline Decay of Kaon (syst) & $\pm 3 \%$ \\
\hline$P_{T}$ spectrum & $\pm 3.3 \%$ \\
\hline Effect of Excites States $\left(B^{+} \rightarrow J / \psi K^{+}\right)$(syst) & $\pm 2.7 \%$ \\
\hline Effect of Excites States $\left(B^{0} \rightarrow J / \psi K^{0}\right)$ (syst) & $\pm 1.1 \%$ \\
\hline \hline
\end{tabular}

Table B.4: Numbers that go into the calculation of the matrix elements $(1,3)$ and $(3,1)$ with same momentum cuts on the bottom and strange mesons. 


\begin{tabular}{|l|c|}
\hline \hline Variable & Value \\
\hline \hline $\mathrm{N}\left(B^{+} \rightarrow J / \psi K^{+}\right)$ & $153.5 \pm 18.9$ \\
\hline $\mathrm{N}\left(B^{0} \rightarrow J / \psi K^{*}(892)^{0}\right)$ & $119.4 \pm 20.1$ \\
\hline$\epsilon_{M C}\left(B^{+} \rightarrow J / \psi K^{+}\right)$ & $0.663 \%$ \\
\hline$\epsilon_{M C}\left(B^{0} \rightarrow J / \psi K^{*}(892)^{0}\right)$ & $0.334 \%$ \\
\hline$\epsilon_{c \tau(B)}\left(B^{+} \rightarrow J / \psi K^{+}\right)$ & $(90.0 \pm 0.5) \%$ \\
\hline$\epsilon_{c \tau(B)}\left(B^{0} \rightarrow J / \psi K^{*}(892)^{0}\right)$ & $(89.3 \pm 0.6) \%$ \\
\hline$\epsilon(K \pi$ tracking) & $(97.9 \pm 0.7) \%$ \\
\hline Helicity (syst) & $\pm 2.5 \%$ \\
\hline Monte Carlo (syst) & $\pm 5 \%$ \\
\hline$C . L .(B$ vertex) (syst) & $\pm 1 \%$ \\
\hline$P_{T}$ spectrum & $\pm 3.3 \%$ \\
\hline Effect of Excites States $\left(B^{+} \rightarrow J / \psi K^{+}\right)($syst) & $\pm 2.7 \%$ \\
\hline Effect of Excites States $\left(B^{0} \rightarrow J / \psi K^{*}(892)^{0}\right)$ (syst) & $\pm 1.5 \%$ \\
\hline \hline
\end{tabular}

Table B.5: Numbers that go into the calculation of the matrix elements $(1,4)$ and $(4,1)$ with different momentum cuts on the bottom and strange mesons.

\begin{tabular}{|c|c|}
\hline Variable & Value \\
\hline$\overline{\mathrm{N}\left(B^{+} \rightarrow J / \psi K^{+}\right)}$ & $\overline{123.3 \pm 15.4}$ \\
\hline $\mathrm{N}\left(B^{0} \rightarrow J / \psi K^{*}(892)^{0}\right)$ & $119.4 \pm 20.1$ \\
\hline$\epsilon_{M C}\left(B^{+} \rightarrow J / \psi K^{+}\right)$ & $0.584 \%$ \\
\hline$\epsilon_{M C}\left(B^{0} \rightarrow J / \psi K^{*}(892)^{0}\right)$ & $0.334 \%$ \\
\hline$\epsilon_{c \tau(B)}\left(B^{+} \rightarrow J / \psi K^{+}\right)$ & $(90.0 \pm 0.5) \%$ \\
\hline$\epsilon_{\mathcal{c \tau}(B)}\left(B^{0} \rightarrow J / \psi K^{*}(892)^{0}\right)$ & $(89.3 \pm 0.6) \%$ \\
\hline$\epsilon(K \pi$ tracking $)$ & $(97.9 \pm 0.7) \%$ \\
\hline Helicity (syst) & $\pm 2.5 \%$ \\
\hline Monte Carlo (syst) & $\pm 5 \%$ \\
\hline C.L. $(B$ vertex $)$ (syst) & $\pm 1 \%$ \\
\hline$P_{T}$ spectrum & $\pm 3.8 \%$ \\
\hline Effect of Excites States $\left(B^{+} \rightarrow J / \psi K^{+}\right)$(syst) & $\pm 2.7 \%$ \\
\hline Effect of Excites States $\left(B^{0} \rightarrow J / \psi K^{*}(892)^{0}\right)$ (syst) & $\pm 1.5 \%$ \\
\hline
\end{tabular}

Table B.6: Numbers that go into the calculation of the matrix elements $(1,4)$ and $(4,1)$ with same momentum cuts on the bottom and strange mesons. 


\begin{tabular}{|l|c|}
\hline \hline Variable & Value \\
\hline \hline $\mathrm{N}\left(B^{+} \rightarrow J / \psi K^{+}\right)$ & $153.5 \pm 18.9$ \\
\hline $\mathrm{N}\left(B_{s}^{0} \rightarrow J / \psi \phi(1020)\right)$ & $26.7 \pm 7.3$ \\
\hline$\epsilon_{M C}\left(B^{+} \rightarrow J / \psi K^{+}\right)$ & $0.663 \%$ \\
\hline$\epsilon_{M C}\left(B_{s}^{0} \rightarrow J / \psi \phi(1020)\right)$ & $0.313 \%$ \\
\hline$\epsilon_{c \tau(B)}\left(B^{+} \rightarrow J / \psi K^{+}\right)$ & $(90.0 \pm 0.5) \%$ \\
\hline$\epsilon_{c \tau(B)}\left(B_{s}^{0} \rightarrow J / \psi \phi(1020)\right)$ & $(88.4 \pm 2.0) \%$ \\
\hline$\epsilon(K \pi$ tracking) & $(97.9 \pm 0.7) \%$ \\
\hline$\epsilon(\phi(1020)$ tracking) & $(90.4 \pm 2.1) \%$ \\
\hline Helicity (syst) & $\pm 2.5 \%$ \\
\hline Monte Carlo (syst) & $\pm 5 \%$ \\
\hline$C . L .(B$ vertex) (syst) & $\pm 1 \%$ \\
\hline Decay of Kaon (syst) & $\pm 3 \%$ \\
\hline$P_{T}$ spectrum & $\pm 3.4 \%$ \\
\hline $\mathcal{B}(\phi(1020))$ (syst) & $\pm 1.8 \%$ \\
\hline Effect of Excites States $\left(B^{+} \rightarrow J / \psi K^{+}\right)$(syst) & $\pm 2.7 \%$ \\
\hline Effect of Excites States $\left(B_{s}^{0} \rightarrow J / \psi \phi(1020)\right)$ (syst) & $\pm 3.7 \%$ \\
\hline \hline
\end{tabular}

Table B.7: Numbers that go into the calculation of the matrix elements $(1,5)$ and $(5,1)$ with different momentum cuts on the bottom and strange mesons. 


\begin{tabular}{|l|c|}
\hline \hline Variable & Value \\
\hline \hline $\mathrm{N}\left(B^{+} \rightarrow J / \psi K^{+}\right)$ & $123.3 \pm 15.4$ \\
\hline $\mathrm{N}\left(B_{s}^{0} \rightarrow J / \psi \phi(1020)\right)$ & $24.7 \pm 6.8$ \\
\hline$\epsilon_{M C}\left(B^{+} \rightarrow J / \psi K^{+}\right)$ & $0.584 \%$ \\
\hline$\epsilon_{M C}\left(B_{s}^{0} \rightarrow J / \psi \phi(1020)\right)$ & $0.278 \%$ \\
\hline$\epsilon_{c \tau}(B)\left(B^{+} \rightarrow J / \psi K^{+}\right)$ & $(90.0 \pm 0.5) \%$ \\
\hline$\epsilon_{c \tau(B)}\left(B_{s}^{0} \rightarrow J / \psi \phi(1020)\right)$ & $(88.4 \pm 2.0) \%$ \\
\hline$\epsilon(K \pi$ tracking) & $(97.9 \pm 0.7) \%$ \\
\hline$\epsilon(\phi(1020)$ tracking) & $(90.4 \pm 2.1) \%$ \\
\hline Helicity (syst) & $\pm 2.5 \%$ \\
\hline Monte Carlo (syst) & $\pm 5 \%$ \\
\hline$C . L .(B$ vertex) (syst) & $\pm 1 \%$ \\
\hline Decay of Kaon (syst) & $\pm 3 \%$ \\
\hline$P_{T}$ spectrum & $\pm 2.3 \%$ \\
\hline $\mathcal{B}(\phi(1020))$ (syst) & $\pm 1.8 \%$ \\
\hline Effect of Excites States $\left(B^{+} \rightarrow J / \psi K^{+}\right)$(syst) & $\pm 2.7 \%$ \\
\hline Effect of Excites States $\left(B_{s}^{0} \rightarrow J / \psi \phi(1020)\right)$ (syst) & $\pm 3.7 \%$ \\
\hline \hline
\end{tabular}

Table B.8: Numbers that go into the calculation of the matrix elements $(1,5)$ and $(5,1)$ with same momentum cuts on the bottom and strange mesons.

\begin{tabular}{|l|c|}
\hline \hline Variable & Value \\
\hline \hline $\mathrm{N}\left(B^{+} \rightarrow J / \psi K^{*}(892)^{+}\right)$ & $21.3 \pm 6.1$ \\
\hline $\mathrm{N}\left(B^{0} \rightarrow J / \psi K^{0}\right)$ & $36.9 \pm 7.3$ \\
\hline$\epsilon_{M C}\left(B^{+} \rightarrow J / \psi K^{*}(892)^{+}\right)$ & $0.0619 \%$ \\
\hline$\epsilon_{M C}\left(B^{0} \rightarrow J / \psi K^{0}\right)$ & $0.177 \%$ \\
\hline$\epsilon_{c \tau(B)}\left(B^{+} \rightarrow J / \psi K^{*}(892)^{+}\right)$ & $(88.0 \pm 0.6) \%$ \\
\hline$\epsilon_{c \tau(B)}\left(B^{0} \rightarrow J / \psi K^{0}\right)$ & $(87.6 \pm 0.7) \%$ \\
\hline$\epsilon(K \pi$ tracking) & $(97.9 \pm 0.7) \%$ \\
\hline Helicity (syst) & $\pm 2.5 \%$ \\
\hline Monte Carlo (syst) & $\pm 5 \%$ \\
\hline$C . L .(B$ vertex) (syst) & $\pm 1 \%$ \\
\hline$P_{T}$ spectrum & $\pm 1.0 \%$ \\
\hline Effect of Excites States $\left(B^{+} \rightarrow J / \psi K^{*}(892)^{+}\right)($syst $)$ & $\pm 2.0 \%$ \\
\hline Effect of Excites States $\left(B^{0} \rightarrow J / \psi K^{0}\right)($ syst $)$ & $\pm 1.1 \%$ \\
\hline \hline
\end{tabular}

Table B.9: Numbers that go into the calculation of the matrix elements $(2,3)$ and $(3,2)$ with different momentum cuts on the bottom and strange mesons. 


\begin{tabular}{|l|c|}
\hline \hline Variable & Value \\
\hline \hline $\mathrm{N}\left(B^{+} \rightarrow J / \psi K^{*}(892)^{+}\right)$ & $20.0 \pm 5.9$ \\
\hline $\mathrm{N}\left(B^{0} \rightarrow J / \psi K^{0}\right)$ & $25.5 \pm 5.7$ \\
\hline$\epsilon_{M C}\left(B^{+} \rightarrow J / \psi K^{*}(892)^{+}\right)$ & $0.0588 \%$ \\
\hline$\epsilon_{M C}\left(B^{0} \rightarrow J / \psi K^{0}\right)$ & $0.136 \%$ \\
\hline$\epsilon_{c \tau(B)}\left(B^{+} \rightarrow J / \psi K^{*}(892)^{+}\right)$ & $(88.0 \pm 0.6) \%$ \\
\hline$\epsilon_{c \tau(B)}\left(B^{0} \rightarrow J / \psi K^{0}\right)$ & $(87.6 \pm 0.7) \%$ \\
\hline$\epsilon(K \pi$ tracking) & $(97.9 \pm 0.7) \%$ \\
\hline Helicity (syst) & $\pm 2.5 \%$ \\
\hline Monte Carlo (syst) & $\pm 5 \%$ \\
\hline$C . L .(B$ vertex) (syst) & $\pm 1 \%$ \\
\hline$P_{T}$ spectrum & $\pm 1.0 \%$ \\
\hline Effect of Excites States $\left(B^{+} \rightarrow J / \psi K^{*}(892)^{+}\right)$(syst) & $\pm 2.0 \%$ \\
\hline Effect of Excites States $\left(B^{0} \rightarrow J / \psi K^{0}\right)($ syst $)$ & $\pm 1.1 \%$ \\
\hline \hline
\end{tabular}

Table B.10: Numbers that go into the calculation of the matrix elements $(2,3)$ and $(3,2)$ with same momentum cuts on the bottom and strange mesons. 


\begin{tabular}{|l|c|}
\hline \hline Variable & Value \\
\hline \hline $\mathrm{N}\left(B^{+} \rightarrow J / \psi K^{*}(892)^{+}\right)$ & $21.3 \pm 6.1$ \\
\hline $\mathrm{N}\left(B^{0} \rightarrow J / \psi K^{*}(892)^{0}\right)$ & $119.4 \pm 20.1$ \\
\hline$\epsilon_{M C}\left(B^{+} \rightarrow J / \psi K^{*}(892)^{+}\right)$ & $0.0619 \%$ \\
\hline$\epsilon_{M C}\left(B^{0} \rightarrow J / \psi K^{*}(892)^{0}\right)$ & $0.334 \%$ \\
\hline$\epsilon_{c \tau(B)}\left(B^{+} \rightarrow J / \psi K^{*}(892)^{+}\right)$ & $(88.0 \pm 0.6) \%$ \\
\hline$\epsilon_{c \tau(B)}\left(B^{0} \rightarrow J / \psi K^{*}(892)^{0}\right)$ & $(89.3 \pm 0.6) \%$ \\
\hline$\epsilon_{c \tau\left(K_{s}\right)}$ & $(95.8 \pm 0.7) \%$ \\
\hline$\epsilon\left(C . L . K_{s}^{0}\right.$ vertex) & $(93.8 \pm 1.7) \%$ \\
\hline$\epsilon\left(C . L . K_{s}^{0}\right.$ mass and vertex) & $(98.3 \pm 0.6) \%$ \\
\hline$\epsilon(K \pi$ tracking) & $(97.9 \pm 0.7) \%$ \\
\hline$\epsilon\left(K_{s}^{0}\right.$ tracking) & $(89.5 \pm 1.0) \%$ \\
\hline Helicity (syst) & $\pm 2.5 \% \times 2$ \\
\hline Monte Carlo (syst) & $\pm 5 \%$ \\
\hline$C . L .(B$ vertex) (syst) & $\pm 1 \%$ \\
\hline Decay of Kaon (syst) & $\pm 3 \%$ \\
\hline$P_{T}$ spectrum & $\pm 1.3 \%$ \\
\hline Effect of Excites States $\left(B^{+} \rightarrow J / \psi K^{*}(892)^{+}\right)$(syst) & $\pm 2.0 \%$ \\
\hline Effect of Excites States $\left(B^{0} \rightarrow J / \psi K^{*}(892)^{0}\right)$ (syst) & $\pm 1.5 \%$ \\
\hline \hline \multicolumn{2}{|l}{}
\end{tabular}

Table B.11: Numbers that go into the calculation of the matrix elements $(2,4)$ and $(4,2)$ with different momentum cuts on the bottom and strange mesons. 


\begin{tabular}{|l|c|}
\hline Variable & Value \\
\hline \hline $\mathrm{N}\left(B^{+} \rightarrow J / \psi K^{*}(892)^{+}\right)$ & $20.0 \pm 5.9$ \\
\hline $\mathrm{N}\left(B^{0} \rightarrow J / \psi K^{*}(892)^{0}\right)$ & $119.4 \pm 20.1$ \\
\hline$\epsilon_{M C}\left(B^{+} \rightarrow J / \psi K^{*}(892)^{+}\right)$ & $0.0588 \%$ \\
\hline$\epsilon_{M C}\left(B^{0} \rightarrow J / \psi K^{*}(892)^{0}\right)$ & $0.334 \%$ \\
\hline$\epsilon_{c \tau(B)}\left(B^{+} \rightarrow J / \psi K^{*}(892)^{+}\right)$ & $(88.0 \pm 0.6) \%$ \\
\hline$\epsilon_{c \tau(B)}\left(B^{0} \rightarrow J / \psi K^{*}(892)^{0}\right)$ & $(89.3 \pm 0.6) \%$ \\
\hline$\epsilon_{c \tau\left(K_{s}\right)}$ & $(95.8 \pm 0.7) \%$ \\
\hline$\epsilon\left(C . L . K_{s}^{0}\right.$ vertex) & $(93.8 \pm 1.7) \%$ \\
\hline$\epsilon\left(C . L . K_{s}^{0}\right.$ mass and vertex) & $(98.3 \pm 0.6) \%$ \\
\hline$\epsilon(K \pi$ tracking) & $(97.9 \pm 0.7) \%$ \\
\hline$\epsilon\left(K_{s}^{0}\right.$ tracking) & $(89.5 \pm 1.0) \%$ \\
\hline Helicity (syst) & $\pm 2.5 \% \times 2$ \\
\hline Monte Carlo (syst) & $\pm 5 \%$ \\
\hline$C . L .(B$ vertex) (syst) & $\pm 1 \%$ \\
\hline Decay of Kaon (syst) & $\pm 3 \%$ \\
\hline$P_{T}$ spectrum & $\pm 1.0 \%$ \\
\hline Effect of Excites States $\left(B^{+} \rightarrow J / \psi K^{*}(892)^{+}\right)$(syst) & $\pm 2.0 \%$ \\
\hline Effect of Excites States $\left(B^{0} \rightarrow J / \psi K^{*}(892)^{0}\right)$ (syst) & $\pm 1.5 \%$ \\
\hline \hline
\end{tabular}

Table B.12: Numbers that go into the calculation of the matrix elements $(2,4)$ and $(4,2)$ with same momentum cuts on the bottom and strange mesons. 


\begin{tabular}{|l|c|}
\hline \hline Variable & Value \\
\hline \hline $\mathrm{N}\left(B^{+} \rightarrow J / \psi K^{*}(892)^{+}\right)$ & $21.3 \pm 6.1$ \\
\hline $\mathrm{N}\left(B_{s}^{0} \rightarrow J / \psi \phi(1020)\right)$ & $26.7 \pm 7.3$ \\
\hline$\epsilon_{M C}\left(B^{+} \rightarrow J / \psi K^{*}(892)^{+}\right)$ & $0.0619 \%$ \\
\hline$\epsilon_{M C}\left(B_{s}^{0} \rightarrow J / \psi \phi(1020)\right)$ & $0.313 \%$ \\
\hline$\epsilon_{c \tau}(B)\left(B^{+} \rightarrow J / \psi K^{*}(892)^{+}\right)$ & $(88.0 \pm 0.6) \%$ \\
\hline$\epsilon_{c \tau}(B)\left(B_{s}^{0} \rightarrow J / \psi \phi(1020)\right)$ & $(88.4 \pm 2.0) \%$ \\
\hline$\epsilon_{c \tau}\left(K_{s}\right)$ & $(95.8 \pm 0.7) \%$ \\
\hline$\epsilon\left(C . L . K_{s}^{0}\right.$ vertex) & $(93.8 \pm 1.7) \%$ \\
\hline$\epsilon\left(C . L . K_{s}^{0}\right.$ mass and vertex) & $(98.3 \pm 0.6) \%$ \\
\hline$\epsilon(K \pi$ tracking) & $(97.9 \pm 0.7) \%$ \\
\hline$\epsilon\left(K_{s}^{0}\right.$ tracking) & $(89.5 \pm 1.0) \%$ \\
\hline$\epsilon(\phi(1020)$ tracking) & $(90.4 \pm 2.1) \%$ \\
\hline Helicity (syst) & $\pm 2.5 \% \times 2$ \\
\hline Monte Carlo (syst) & $\pm 5 \%$ \\
\hline$C . L .(B$ vertex) (syst) & $\pm 1 \%$ \\
\hline Decay of Kaon (syst) & $\pm 3 \% \times 2$ \\
\hline$P_{T}$ spectrum & $\pm 1.2 \%$ \\
\hline $\mathcal{B}(\phi(1020))$ (syst) & $\pm 1.8 \%$ \\
\hline Effect of Excites States $\left(B^{+} \rightarrow J / \psi K^{*}(892)^{+}\right)$(syst) & $\pm 2.0 \%$ \\
\hline Effect of Excites States $\left(B_{s}^{0} \rightarrow J / \psi \phi(1020)\right)$ (syst) & $\pm 3.7 \%$ \\
\hline \hline
\end{tabular}

Table B.13: Numbers that go into the calculation of the matrix elements $(2,5)$ and $(5,2)$ with different momentum cuts on the bottom and strange mesons. 


\begin{tabular}{|l|c|}
\hline \hline Variable & Value \\
\hline \hline $\mathrm{N}\left(B^{+} \rightarrow J / \psi K^{*}(892)^{+}\right)$ & $20.0 \pm 5.9$ \\
\hline $\mathrm{N}\left(B_{d} \rightarrow J / \psi \phi\right)$ & $24.7 \pm 6.8$ \\
\hline$\epsilon_{M C}\left(B^{+} \rightarrow J / \psi K^{*}(892)^{+}\right)$ & $0.0588 \%$ \\
\hline$\epsilon_{M C}\left(B_{s}^{0} \rightarrow J / \psi \phi(1020)\right)$ & $0.278 \%$ \\
\hline$\epsilon_{c \tau(B)}\left(B^{+} \rightarrow J / \psi K^{*}(892)^{+}\right)$ & $(88.0 \pm 0.6) \%$ \\
\hline$\epsilon_{\mathrm{cr}(B)}\left(B_{s}^{0} \rightarrow J / \psi \phi(1020)\right)$ & $(88.4 \pm 2.0) \%$ \\
\hline$\left.\epsilon_{c \tau(K s}\right)$ & $(95.8 \pm 0.7) \%$ \\
\hline$\epsilon\left(C . L . K_{s}^{0}\right.$ vertex) & $(93.8 \pm 1.7) \%$ \\
\hline$\epsilon\left(C . L . K_{s}^{0}\right.$ mass and vertex) & $(98.3 \pm 0.6) \%$ \\
\hline$\epsilon(K \pi$ tracking) & $(97.9 \pm 0.7) \%$ \\
\hline$\epsilon\left(K_{s}^{0}\right.$ tracking) & $(89.5 \pm 1.0) \%$ \\
\hline$\epsilon(\phi(1020)$ tracking) & $(90.4 \pm 2.1) \%$ \\
\hline Helicity (syst) & $\pm 2.5 \% \times 2$ \\
\hline Monte Carlo (syst) & $\pm 5 \%$ \\
\hline$C . L .(B$ vertex) (syst) & $\pm 1 \%$ \\
\hline Decay of Kaon (syst) & $\pm 3 \% \times 2$ \\
\hline$P_{T}$ spectrum & $\pm 2.4 \%$ \\
\hline $\mathcal{B}(\phi(1020))$ (syst) & $\pm 1.8 \%$ \\
\hline Effect of Excites States $\left(B^{+} \rightarrow J / \psi K^{*}(892)^{+}\right)$(syst) & $\pm 2.0 \%$ \\
\hline Effect of Excites States $\left(B_{s}^{0} \rightarrow J / \psi \phi(1020)\right)$ (syst) & $\pm 3.7 \%$ \\
\hline \hline
\end{tabular}

Table B.14: Numbers that go into the calculation of the matrix elements $(2,5)$ and $(5,2)$ with same momentum cuts on the bottom and strange mesons. 


\begin{tabular}{|l|c|}
\hline \hline Variable & Value \\
\hline \hline $\mathrm{N}\left(B^{0} \rightarrow J / \psi K^{0}\right)$ & $36.9 \pm 7.3$ \\
\hline $\mathrm{N}\left(B^{0} \rightarrow J / \psi K^{*}(892)^{0}\right)$ & $119.4 \pm 20.1$ \\
\hline$\epsilon_{M C}\left(B^{0} \rightarrow J / \psi K^{0}\right)$ & $0.177 \%$ \\
\hline$\epsilon_{M C}\left(B^{0} \rightarrow J / \psi K^{*}(892)^{0}\right)$ & $0.334 \%$ \\
\hline$\epsilon_{c \tau(B)}\left(B^{0} \rightarrow J / \psi K^{0}\right)$ & $(87.6 \pm 0.7) \%$ \\
\hline$\epsilon_{c \tau(B)}\left(B^{0} \rightarrow J / \psi K^{*}(892)^{0}\right)$ & $(89.3 \pm 0.6) \%$ \\
\hline$\epsilon_{c \tau}\left(K_{s}\right)$ & $(95.8 \pm 0.7) \%$ \\
\hline$\epsilon\left(C . L . K_{s}^{0}\right.$ vertex) & $(93.8 \pm 1.7) \%$ \\
\hline$\epsilon\left(C . L . K_{s}^{0}\right.$ mass and vertex) & $(98.3 \pm 0.6) \%$ \\
\hline$\epsilon(K \pi$ tracking) & $(97.9 \pm 0.7) \% \times 2$ \\
\hline$\epsilon\left(K_{s}^{0}\right.$ tracking) & $(89.5 \pm 1.0) \%$ \\
\hline Helicity (syst) & $\pm 2.5 \%$ \\
\hline Monte Carlo (syst) & $\pm 5 \%$ \\
\hline$C . L .(B$ vertex) (syst) & $\pm 1 \%$ \\
\hline Decay of Kaon (syst) & $\pm 3 \%$ \\
\hline$P_{T}$ spectrum & $\pm 1.5 \%$ \\
\hline Effect of Excites States $\left(B^{0} \rightarrow J / \psi K^{0}\right)($ syst) & $\pm 1.1 \%$ \\
\hline Effect of Excites States $\left(B^{0} \rightarrow J / \psi K^{*}(892)^{0}\right)($ syst $)$ & $\pm 1.5 \%$ \\
\hline \hline
\end{tabular}

Table B.15: Numbers that go into the calculation of the matrix elements $(3,4)$ and $(4,3)$ with different momentum cuts on the bottom and strange mesons. 


\begin{tabular}{|l|c|}
\hline \hline Variable & Value \\
\hline \hline $\mathrm{N}\left(B^{0} \rightarrow J / \psi K^{0}\right)$ & $25.5 \pm 5.7$ \\
\hline $\mathrm{N}\left(B^{0} \rightarrow J / \psi K^{*}(892)^{0}\right)$ & $119.4 \pm 20.1$ \\
\hline$\epsilon_{M C}\left(B^{0} \rightarrow J / \psi K^{0}\right)$ & $0.136 \%$ \\
\hline$\epsilon_{M C}\left(B^{0} \rightarrow J / \psi K^{*}(892)^{0}\right)$ & $0.334 \%$ \\
\hline$\epsilon_{c \tau}(B)\left(B^{0} \rightarrow J / \psi K^{0}\right)$ & $(87.6 \pm 0.7) \%$ \\
\hline$\epsilon_{c \tau(B)}\left(B^{0} \rightarrow J / \psi K^{*}(892)^{0}\right)$ & $(89.3 \pm 0.6) \%$ \\
\hline$\epsilon_{c \tau}\left(K_{s}\right)$ & $(95.8 \pm 0.7) \%$ \\
\hline$\epsilon\left(C . L . K_{s}^{0}\right.$ vertex $)$ & $(93.8 \pm 1.7) \%$ \\
\hline$\epsilon\left(C . L . K_{s}^{0}\right.$ mass and vertex $)$ & $(98.3 \pm 0.6) \%$ \\
\hline$\epsilon(K \pi$ tracking) & $(97.9 \pm 0.7) \% \times 2$ \\
\hline$\epsilon\left(K_{s}^{0}\right.$ tracking) & $(89.5 \pm 1.0) \%$ \\
\hline Helicity (syst) & $\pm 2.5 \%$ \\
\hline Monte Carlo (syst) & $\pm 5 \%$ \\
\hline$C . L .(B$ vertex) (syst) & $\pm 1 \%$ \\
\hline Decay of Kaon (syst) & $\pm 3 \%$ \\
\hline$P_{T}$ spectrum & $\pm 1.0 \%$ \\
\hline Effect of Excites States $\left(B^{0} \rightarrow J / \psi K^{0}\right)($ syst $)$ & $\pm 1.1 \%$ \\
\hline Effect of Excites States $\left(B^{0} \rightarrow J / \psi K^{*}(892)^{0}\right)($ syst $)$ & $\pm 1.5 \%$ \\
\hline \hline
\end{tabular}

Table B.16: Numbers that go into the calculation of the matrix elements $(3,4)$ and $(4,3)$ with same momentum cuts on the bottom and strange mesons. 


\begin{tabular}{|l|c|}
\hline \hline Variable & Value \\
\hline \hline $\mathrm{N}\left(B^{0} \rightarrow J / \psi K^{0}\right)$ & $36.9 \pm 7.3$ \\
\hline $\mathrm{N}\left(B_{s}^{0} \rightarrow J / \psi \phi(1020)\right)$ & $26.7 \pm 7.3$ \\
\hline$\epsilon_{M C}\left(B^{0} \rightarrow J / \psi K^{0}\right)$ & $0.177 \%$ \\
\hline$\epsilon_{M C}\left(B_{s}^{0} \rightarrow J / \psi \phi(1020)\right)$ & $0.313 \%$ \\
\hline$\epsilon_{c \tau(B)}\left(B^{0} \rightarrow J / \psi K^{0}\right)$ & $(87.6 \pm 0.7) \%$ \\
\hline$\epsilon_{c \tau(B)}\left(B_{s}^{0} \rightarrow J / \psi \phi(1020)\right)$ & $(88.4 \pm 2.0) \%$ \\
\hline$\epsilon_{c \tau\left(K_{s}\right)} K^{0}$ vertex) & $(95.8 \pm 0.7) \%$ \\
\hline$\epsilon\left(C . L . K_{s}\right)$ & $(93.8 \pm 1.7) \%$ \\
\hline$\epsilon\left(C . L . K_{s}^{0}\right.$ mass and vertex) & $(98.3 \pm 0.6) \%$ \\
\hline$\epsilon\left(K_{s}^{0}\right.$ tracking) & $(89.5 \pm 1.0) \%$ \\
\hline$\epsilon(\phi(1020)$ tracking) & $(90.4 \pm 2.1) \%$ \\
\hline Helicity (syst) & $\pm 2.5 \%$ \\
\hline Monte Carlo (syst) & $\pm 5 \%$ \\
\hline$C . L .(B$ vertex) (syst) & $\pm 1 \%$ \\
\hline Decay of Kaon (syst) & $\pm 3 \% \times 2$ \\
\hline$P_{T}$ spectrum & $\pm 1.4 \%$ \\
\hline $\mathcal{B}(\phi(1020))$ (syst) & $\pm 1.8 \%$ \\
\hline Effect of Excites States $\left(B^{0} \rightarrow J / \psi K^{0}\right)$ (syst) & $\pm 1.1 \%$ \\
\hline Effect of Excites States $\left(B_{s}^{0} \rightarrow J / \psi \phi(1020)\right)$ (syst) & $\pm 3.7 \%$ \\
\hline \hline
\end{tabular}

Table B.17: Numbers that go into the calculation of the matrix elements $(3,5)$ and $(5,3)$ with different momentum cuts on the bottom and strange mesons. 


\begin{tabular}{|l|c|}
\hline \hline Variable & Value \\
\hline \hline $\mathrm{N}\left(B^{0} \rightarrow J / \psi K^{0}\right)$ & $25.5 \pm 5.7$ \\
\hline $\mathrm{N}\left(B_{s}^{0} \rightarrow J / \psi \phi(1020)\right)$ & $24.7 \pm 6.8$ \\
\hline$\epsilon_{M C}\left(B^{0} \rightarrow J / \psi K^{0}\right)$ & $0.136 \%$ \\
\hline$\epsilon_{M C}\left(B_{s}^{0} \rightarrow J / \psi \phi(1020)\right)$ & $0.278 \%$ \\
\hline$\epsilon_{c \tau(B)}\left(B^{0} \rightarrow J / \psi K^{0}\right)$ & $(87.6 \pm 0.7) \%$ \\
\hline$\epsilon_{c \tau(B)}\left(B_{s}^{0} \rightarrow J / \psi \phi(1020)\right)$ & $(88.4 \pm 2.0) \%$ \\
\hline$\epsilon_{c \tau\left(K_{s}\right)} K_{s}^{0}$ vertex) & $(95.8 \pm 0.7) \%$ \\
\hline$\epsilon\left(C . L . K_{s}^{0}\right.$ mass and vertex) & $(93.8 \pm 1.7) \%$ \\
\hline$\epsilon\left(C . L . K^{0}\right)$ & $(98.3 \pm 0.6) \%$ \\
\hline$\epsilon\left(K_{s}^{0}\right.$ tracking) & $(89.5 \pm 1.0) \%$ \\
\hline$\epsilon(\phi(1020)$ tracking) & $(90.4 \pm 2.1) \%$ \\
\hline Helicity (syst) & $\pm 2.5 \%$ \\
\hline Monte Carlo (syst) & $\pm 5 \%$ \\
\hline$C . L .(B$ vertex) (syst) & $\pm 1 \%$ \\
\hline Decay of Kaon (syst) & $\pm 3 \% \times 2$ \\
\hline$P_{T}$ spectrum & $\pm 1.6 \%$ \\
\hline $\mathcal{B}(\phi(1020))$ (syst) & $\pm 1.8 \%$ \\
\hline Effect of Excites States $\left(B^{0} \rightarrow J / \psi K^{0}\right)$ (syst) & $\pm 1.1 \%$ \\
\hline Effect of Excites States $\left(B_{s}^{0} \rightarrow J / \psi \phi(1020)\right)$ (syst) & $\pm 3.7 \%$ \\
\hline \hline
\end{tabular}

Table B.18: Numbers that go into the calculation of the matrix elements $(3,5)$ and $(5,3)$ with same momentum cuts on the bottom and strange mesons. 


\begin{tabular}{|l|c|}
\hline \hline Variable & Value \\
\hline \hline $\mathrm{N}\left(B^{0} \rightarrow J / \psi K^{*}(892)^{0}\right)$ & $119.4 \pm 20.1$ \\
\hline $\mathrm{N}\left(B_{s}^{0} \rightarrow J / \psi \phi(1020)\right)$ & $26.7 \pm 7.3$ \\
\hline$\epsilon_{M C}\left(B^{0} \rightarrow J / \psi K^{*}(892)^{0}\right)$ & $0.334 \%$ \\
\hline$\epsilon_{M C}\left(B_{s}^{0} \rightarrow J / \psi \phi(1020)\right)$ & $0.313 \%$ \\
\hline$\epsilon_{c \tau(B)}\left(B^{0} \rightarrow J / \psi K^{*}(892)^{0}\right)$ & $(89.3 \pm 0.6) \%$ \\
\hline$\epsilon_{c \tau}(B)\left(B_{s}^{0} \rightarrow J / \psi \phi(1020)\right)$ & $(88.4 \pm 2.0) \%$ \\
\hline$\epsilon(K \pi$ tracking) & $(97.9 \pm 0.7) \% \times 2$ \\
\hline$\epsilon(\phi(1020)$ tracking) & $(90.4 \pm 2.1) \%$ \\
\hline Helicity (syst) & $\pm 2.5 \% \times 2$ \\
\hline Monte Carlo (syst) & $\pm 5 \%$ \\
\hline$C . L .(B$ vertex) (syst) & $\pm 1 \%$ \\
\hline Decay of Kaon (syst) & $\pm 1.0 \%$ \\
\hline$P_{T}$ spectrum & $\pm 1.8 \%$ \\
\hline $\mathcal{B}(\phi(1020))$ (syst) & $\pm 1.5 \%$ \\
\hline Effect of Excites States $\left(B^{0} \rightarrow J / \psi K^{*}(892)^{0}\right)$ (syst) & $\pm 3.7 \%$ \\
\hline Effect of Excites States $\left(B_{s}^{0} \rightarrow J / \psi \phi(1020)\right)$ (syst) & \pm \\
\hline \hline
\end{tabular}

Table B.19: Numbers that go into the calculation of the matrix elements $(4,5)$ and $(5,4)$ with different momentum cuts on the bottom and strange mesons. 


\begin{tabular}{|l|c|}
\hline \hline Variable & Value \\
\hline \hline $\mathrm{N}\left(B^{0} \rightarrow J / \psi K^{*}(892)^{0}\right)$ & $119.4 \pm 20.1$ \\
\hline $\mathrm{N}\left(B_{s}^{0} \rightarrow J / \psi \phi(1020)\right)$ & $24.7 \pm 6.8$ \\
\hline$\epsilon_{M C}\left(B^{0} \rightarrow J / \psi K^{*}(892)^{0}\right)$ & $0.334 \%$ \\
\hline$\epsilon_{M C}\left(B_{s}^{0} \rightarrow J / \psi \phi(1020)\right)$ & $0.278 \%$ \\
\hline$\epsilon_{c \tau(B)}\left(B^{0} \rightarrow J / \psi K^{*}(892)^{0}\right)$ & $(89.3 \pm 0.6) \%$ \\
\hline$\epsilon_{c \tau(B)}\left(B_{s}^{0} \rightarrow J / \psi \phi(1020)\right)$ & $(88.4 \pm 2.0) \%$ \\
\hline$\epsilon(K \pi$ tracking) & $(97.9 \pm 0.7) \% \times 2$ \\
\hline$\epsilon(\phi(1020)$ tracking) & $(90.4 \pm 2.1) \%$ \\
\hline Helicity (syst) & $\pm 2.5 \% \times 2$ \\
\hline Monte Carlo (syst) & $\pm 5 \%$ \\
\hline$C . L .(B$ vertex) (syst) & $\pm 1 \%$ \\
\hline Decay of Kaon (syst) & $\pm 3 \%$ \\
\hline$P_{T}$ spectrum & $\pm 2.1 \%$ \\
\hline $\mathcal{B}(\phi(1020))$ (syst) & $\pm 1.8 \%$ \\
\hline Effect of Excites States $\left(B^{0} \rightarrow J / \psi K^{*}(892)^{0}\right)$ (syst) & $\pm 1.5 \%$ \\
\hline Effect of Excites States $\left(B_{s}^{0} \rightarrow J / \psi \phi(1020)\right)$ (syst) & $\pm 3.7 \%$ \\
\hline \hline
\end{tabular}

Table B.20: Numbers that go into the calculation of the matrix elements $(4,5)$ and $(5,4)$ with same momentum cuts on the bottom and strange mesons. 


\section{Appendix C}

\section{The CDF Collaboration}

The design, construction and operation of the Collider Detector at Fermilab has involved the hard work of many dedicated people. These include technicians, assistants, engineers and physicists from the 35 institutions that are listed below. In particular, the last collider run would not have shed light on many aspects of particle physics had it not been for the cooperation of the members of the CDF collaboration whose names are listed below. 
F. Abe ${ }^{14}$ H. Akimoto, ${ }^{32}$ A. Akopian, ${ }^{27}$ M. G. Albrow ${ }^{7}$ S. R. Amendolia, ${ }^{23}$ D. Amidei,${ }^{17} \mathrm{~J}$. Antos,${ }^{29} \mathrm{C}$. Anway-Wiese,${ }^{4} \mathrm{~S}$. Aota,${ }^{32}$ G. Apollinari,${ }^{27}$ T. Asakawa ${ }^{32}$ W. Ashmanskas, ${ }^{15}$ M. Atac,${ }^{7}$ F. Azfar, ${ }^{22}$ P. Azzi-Bacchetta,,${ }^{21}$ N. Bacchetta,${ }^{21}$ W. Badgett, ${ }^{17}$ S. Bagdasarov, ${ }^{27}$ M. W. Bailey, ${ }^{19}$ J. Bao, ${ }^{35}$ P. de Barbaro, ${ }^{26}$ A. Barbaro-Galtieri, ${ }^{15}$ V. E. Barnes,${ }^{25}$ B. A. Barnett,${ }^{13}$ E. Barzi ${ }^{8}$ G. Bauer, ${ }^{16}$ T. Baumann,${ }^{9}$ F. Bedeschi, ${ }^{23}$ S. Behrends, ${ }^{3}$ S. Belforte,${ }^{23}$ G. Bellettini, ${ }^{23}$ J. Bellinger ${ }^{34}$ D. Benjamin, ${ }^{31}$ J. Benlloch, ${ }^{16}$ J. Bensinger ${ }^{3}$ D. Benton, ${ }^{22}$ A. Beretvas, ${ }^{7}$ J. P. Berge, ${ }^{7}$ J. Berryhill, ${ }^{5}$ S. Bertolucci, ${ }^{8}$ B. Bevensee, ${ }^{22}$ A. Bhatti, ${ }^{27}$ K. Biery, ${ }^{12}$ M. Binkley,${ }^{7}$ D. Bisello, ${ }^{21}$ R. E. Blair,${ }^{1}$ C. Blocker,${ }^{3}$ A. Bodek,${ }^{26}$ W. Bokhari, ${ }^{16}$ G. Bolla,${ }^{21}$ V. Bolognesi ${ }^{7}$ D. Bortoletto, ${ }^{25}$ J. Boudreau ${ }^{24}$ L. Breccia, ${ }^{2}$ C. Bromberg, ${ }^{18}$ N. Bruner ${ }^{19}$ E. Buckley-Geer, ${ }^{7}$ H. S. Budd,${ }^{26}$ K. Burkett, ${ }^{17}$ G. Busetto, ${ }^{21}$ A. ByonWagner, ${ }^{7}$ K. L. Byrum, ${ }^{1}$ J. Cammerata, ${ }^{13}$ C. Campagnari, ${ }^{7}$ M. Campbell, ${ }^{17}$ A. Caner, ${ }^{7}$ W. Carithers, ${ }^{15}$ D. Carlsmith, ${ }^{34}$ A. Castro, ${ }^{21}$ D. Cauz ${ }^{23}$ Y. Cen,${ }^{26}$ F. Cervelli, ${ }^{23}$ P. S. Chang, ${ }^{29}$ P. T. Chang, ${ }^{29}$ H. Y. Chao, ${ }^{29}$ J. Chapman, ${ }^{17}$ M.-T. Cheng, ${ }^{29}$ G. Chiarelli, ${ }^{23}$ T. Chikamatsu, ${ }^{32}$ C. N. Chiou, ${ }^{29}$ L. Christofek, ${ }^{11}$ S. Cihangir, ${ }^{7}$ A. G. Clark,${ }^{23}$ M. Cobal, ${ }^{23}$ M. Contreras, ${ }^{5}$ J. Conway, ${ }^{28}$ J. Cooper,${ }^{7}$ M. Cordelli, ${ }^{8}$ C. Couyoumtzelis, ${ }^{23}$ D. Crane,${ }^{1}$ D. Cronin-Hennessy,${ }^{6}$ R. Culbertson,${ }^{5}$ J. D. Cunningham, ${ }^{3}$ T. Daniels, ${ }^{16}$ F. DeJongh, ${ }^{7}$ S. Delchamps,${ }^{7}$ S. Dell'Agnello, ${ }^{23}$ M. Dell'Orso, ${ }^{23}$ R. Demina, ${ }^{7}$ L. Demortier, ${ }^{27}$ B. Denby, ${ }^{23}$ M. Deninno, ${ }^{2}$ P. F. Derwent,${ }^{17}$ T. Devlin, ${ }^{28}$ J. R. Dittmann,${ }^{6}$ S. Donati, ${ }^{23}$ J. Done,${ }^{30}$ T. Dorigo, ${ }^{21}$ A. Dunn,,${ }^{17}$ N. Eddy,${ }^{17}$ K. Einsweiler, ${ }^{15}$ J. E. Elias, ${ }^{7}$ R. Ely, ${ }^{15}$ E. Engels, Jr. ${ }^{24}$ D. Errede,${ }^{11}$ S. Errede,${ }^{11}$ Q. Fan,${ }^{26}$ I. Fiori,${ }^{2}$ B. Flaugher, ${ }^{7}$ G. W. Foster,${ }^{7}$ M. Franklin,${ }^{9}$ M. Frautschi, ${ }^{31}$ J. Freeman,${ }^{7}$ J. Friedman,${ }^{16}$ T. A. Fuess, ${ }^{1}$ Y. Fukui, ${ }^{14}$ S. Funaki ${ }^{32}$ G. Gagliardi, ${ }^{23}$ S. Galeotti, ${ }^{23}$ M. Gallinaro, ${ }^{21}$ M. Garcia-Sciveres,${ }^{15}$ A. F. Garfinkel ${ }^{25}$ C. Gay, ${ }^{9}$ S. Geer, ${ }^{7}$ D. W. Gerdes,${ }^{13}$ P. Giannetti, ${ }^{23}$ N. Giokaris, ${ }^{27}$ P. Giromini, ${ }^{8}$ L. Gladney, ${ }^{22}$ D. Glenzinski, ${ }^{13}$ M. Gold,${ }^{19}$ J. Gonzalez, ${ }^{22}$ A. Gordon, ${ }^{9}$ A. T. Goshaw ${ }^{6}$ K. Goulianos, ${ }^{27}$ H. Grassmann, ${ }^{23}$ L. Groer,${ }^{28}$ C. Grosso-Pilcher, ${ }^{5}$ G. Guillian, ${ }^{17}$ R. S. Guo, ${ }^{29}$ C. Haber ${ }^{15}$ E. Hafen,${ }^{16}$ S. R. Hahn, ${ }^{7}$ R. Hamilton, ${ }^{9}$ R. Handler,${ }^{34}$ R. M. Hans ${ }^{35}$ K. Hara, ${ }^{32}$ A. D. Hardman,${ }^{25}$ B. Harral, ${ }^{22}$ R. M. Harris, ${ }^{7}$ S. A. Hauger,${ }^{6}$ J. Hauser, ${ }^{4}$ C. Hawk, ${ }^{28}$ E. Hayashi, ${ }^{32}$ J. Heinrich, ${ }^{22}$ K. D. Hoffman, ${ }^{25}$ M. Hohlmann, ${ }^{1,5}$ C. Holck ${ }^{22}$ R. Hollebeek, ${ }^{22}$ L. Holloway, ${ }^{11}$ A. Hölscher, ${ }^{12}$ S. Hong, ${ }^{17}$ G. Houk, ${ }^{22}$ P. Hu, ${ }^{24}$ B. T. Huffman, ${ }^{24}$ R. Hughes,${ }^{26}$ J. Huston, ${ }^{18}$ J. Huth,${ }^{9}$ J. Hylen,${ }^{7}$ H. Ikeda ${ }^{32}$ M. Incagli, ${ }^{23} \mathrm{~J}$. Incandela, ${ }^{7} \mathrm{G}$. Introzzi, ${ }^{23} \mathrm{~J}$. Iwai, ${ }^{32}$ Y. Iwata, ${ }^{10} \mathrm{H}$. Jensen, ${ }^{7}$ U. Joshi, ${ }^{7}$ 
R. W. Kadel, ${ }^{15}$ E. Kajfasz,${ }^{7 a}$ H. Kambara, ${ }^{23}$ T. Kamon, ${ }^{30}$ T. Kaneko, ${ }^{32}$ K. Karr,${ }^{33}$ H. Kasha, ${ }^{35}$ Y. Kato,${ }^{20}$ T. A. Keaffaber, ${ }^{25}$ L. Keeble, ${ }^{8}$ K. Kelley, ${ }^{16}$ R. D. Kennedy, ${ }^{28}$ R. Kephart ${ }^{7}$ P. Kesten,${ }^{15}$ D. Kestenbaum, ${ }^{9}$ R. M. Keup,${ }^{11}$ H. Keutelian, ${ }^{7}$ F. Keyvan,${ }^{4}$ B. Kharadia, ${ }^{11}$ B. J. Kim, ${ }^{26}$ D. H. Kim,${ }^{7 a}$ H. S. Kim, ${ }^{12}$ S. B. Kim, ${ }^{17}$ S. H. Kim, ${ }^{32}$ Y. K. Kim, ${ }^{15}$ L. Kirsch, ${ }^{3}$ P. Koehn, ${ }^{26}$ K. Kondo, ${ }^{32}$ J. Konigsberg, ${ }^{9}$ S. Kopp ${ }^{5}$ K. Kordas, ${ }^{12}$ A. Korytov, ${ }^{16}$ W. Koska, ${ }^{7}$ E. Kovacs,${ }^{7 a}$ W. Kowald,${ }^{6}$ M. Krasberg, ${ }^{17}$ J. Kroll, ${ }^{7}$ M. Kruse, ${ }^{25}$ T. Kuwabara, ${ }^{32}$ S. E. Kuhlmann, ${ }^{1}$ E. Kuns, ${ }^{28}$ A. T. Laasanen, ${ }^{25}$ N. Labanca, ${ }^{23}$ S. Lammel, ${ }^{7}$ J. I. Lamoureux,${ }^{3}$ T. LeCompte, ${ }^{1}$ S. Leone, ${ }^{23}$ J. D. Lewis ${ }^{7}$ P. Limon, ${ }^{7}$ M. Lindgren, ${ }^{4}$ T. M. Liss,${ }^{11}$ N. Lockyer,${ }^{22}$ O. Long, ${ }^{22}$ C. Loomis,${ }^{28}$ M. Loreti, ${ }^{21}$ J. Lu, ${ }^{30}$ D. Lucchesi,${ }^{23}$ P. Lukens,${ }^{7}$ S. Lusin,${ }^{34}$ J. Lys,${ }^{15}$ K. Maeshima,${ }^{7}$ A. Maghakian, ${ }^{27}$ P. Maksimovic, ${ }^{16}$ M. Mangano, ${ }^{23}$ J. Mansour, ${ }^{18}$ M. Mariotti, ${ }^{21}$ J. P. Marriner ${ }^{7}$ A. Martin, ${ }^{11}$ J. A. J. Matthews, ${ }^{19}$ R. Mattingly,${ }^{16}$ P. McIntyre, ${ }^{30}$ P. Melese,${ }^{27}$ A. Menzione, ${ }^{23}$ E. Meschi, ${ }^{23}$ S. Metzler,${ }^{22}$ C. Miao, ${ }^{17}$ T. Miao, ${ }^{7}$ G. Michail, ${ }^{9}$ R. Miller ${ }^{18}$ H. Minato ${ }^{32}$ S. Miscetti, ${ }^{8}$ M. Mishina,${ }^{14}$ H. Mitsushio, ${ }^{32}$ T. Miyamoto ${ }^{32}$ S. Miyashita, ${ }^{32}$ N. Moggi,${ }^{23}$ Y. Morita,${ }^{14}$ J. Mueller,${ }^{24}$ A. Mukherjee,${ }^{7}$ T. Muller, ${ }^{4}$ P. Murat, ${ }^{23}$ H. Nakada, ${ }^{32}$ I. Nakano, ${ }^{32}$ C. Nelson, ${ }^{7}$ D. Neuberger,${ }^{4}$ C. Newman-Holmes, ${ }^{7}$ M. Ninomiya, ${ }^{32}$ L. Nodulman, ${ }^{1}$ S. H. Oh, ${ }^{6}$ K. E. Ohl, ${ }^{35}$ T. Ohmoto ${ }^{10}$ T. Ohsugi, ${ }^{10}$ R. Oishi, ${ }^{32}$ M. Okabe,${ }^{32}$ T. Okusawa, ${ }^{20}$ R. Oliveira,${ }^{22}$ J. Olsen, ${ }^{34}$ C. Pagliarone,${ }^{2}$ R. Paoletti, ${ }^{23}$ V. Papadimitriou, ${ }^{31}$ S. P. Pappas, ${ }^{35}$ S. Park,${ }^{7}$ A. Parri,${ }^{8}$ J. Patrick, ${ }^{7}$ G. Pauletta,${ }^{23}$ M. Paulini,${ }^{15}$ A. Perazzo, ${ }^{23}$ L. Pescara, ${ }^{21}$ M. D. Peters, ${ }^{15}$ T. J. Phillips,${ }^{6}$ G. Piacentino, ${ }^{2}$ M. Pillai, ${ }^{26}$ K. T. Pitts,${ }^{7}$ R. Plunkett,${ }^{7}$ L. Pondrom,${ }^{34}$ J. Proudfoot, ${ }^{1}$ F. Ptohos, ${ }^{9}$ G. Punzi, ${ }^{23}$ K. Ragan, ${ }^{12}$ A. Ribon, ${ }^{21}$ F. Rimondi, ${ }^{2}$ L. Ristori, ${ }^{23}$ W. J. Robertson, ${ }^{6}$ T. Rodrigo, ${ }^{7 a} \mathrm{~S}$. Rolli, ${ }^{23}$ J. Romano, ${ }^{5}$ L. Rosenson, ${ }^{16}$ R. Roser, ${ }^{11}$ W. K. Sakumoto, ${ }^{26}$ D. Saltzberg, ${ }^{5}$ A. Sansoni ${ }^{8}$ L. Santi, ${ }^{23}$ H. Sato,${ }^{32}$ V. Scarpine, ${ }^{30}$ P. Schlabach,${ }^{9}$ E. E. Schmidt,${ }^{7}$ M. P. Schmidt, ${ }^{35}$ A. Scribano, ${ }^{23}$ S. Segler, ${ }^{7}$ S. Seidel, ${ }^{19}$ Y. Seiya, ${ }^{32}$ G. Sganos,${ }^{12}$ M. D. Shapiro, ${ }^{15}$ N. M. Shaw,${ }^{25}$ Q. Shen ${ }^{25}$ P. F. Shepard, ${ }^{24}$ M. Shimojima, ${ }^{32}$ M. Shochet,${ }^{5}$ J. Siegrist, ${ }^{15}$ A. Sill,${ }^{31}$ P. Sinervo, ${ }^{12}$ P. Singh, ${ }^{24}$ J. Skarha, ${ }^{13}$ K. Sliwa, ${ }^{33}$ F. D. Snider, ${ }^{13}$ T. Song, ${ }^{17}$ J. Spalding, ${ }^{7}$ T. Speer,${ }^{23}$ P. Sphicas, ${ }^{16}$ F. Spinella, ${ }^{23}$ M. Spiropulu, ${ }^{9}$ L. Spiegel, ${ }^{7}$ L. Stanco, ${ }^{21}$ J. Steele,${ }^{34}$ A. Stefanini ${ }^{23}$ K. Strahl,${ }^{12}$ J. Strait, ${ }^{7}$ R. Ströhmer, ${ }^{9}$ D. Stuart, ${ }^{7}$ G. Sullivan, ${ }^{5}$ A. Soumarokov, ${ }^{29}$ K. Sumorok, ${ }^{16}$ J. Suzuki, ${ }^{32}$ T. Takada, ${ }^{32}$ T. Takahashi, ${ }^{20}$ T. Takano, ${ }^{32}$ K. Takikawa, ${ }^{32}$ N. Tamura, ${ }^{10}$ F. Tartarelli, ${ }^{23}$ W. Taylor, ${ }^{12}$ P. K. Teng, ${ }^{29}$ Y. Teramoto, ${ }^{20}$ S. Tether, ${ }^{16}$ D. Theriot,${ }^{7}$ T. L. Thomas, ${ }^{19}$ R. Thun, ${ }^{17}$ M. Timko, ${ }^{33}$ P. Tipton, ${ }^{26}$ A. Titov, ${ }^{27}$ S. Tkaczyk,${ }^{7}$ 
D. Toback, ${ }^{5}$ K. Tollefson, ${ }^{26}$ A. Tollestrup, ${ }^{7}$ J. Tonnison, ${ }^{25}$ J. F. de Troconiz, ${ }^{9}$ S. Truitt,${ }^{17}$ J. Tseng, ${ }^{13}$ N. Turini, ${ }^{23}$ T. Uchida,${ }^{32}$ N. Uemura,${ }^{32}$ F. Ukegawa, ${ }^{22}$ G. Unal, ${ }^{22}$ J. Valls,${ }^{7}$ S. C. van den Brink, ${ }^{24}$ S. Vejcik, III, ${ }^{17}$ G. Velev ${ }^{23}$ R. Vidal, ${ }^{7}$ M. Vondracek, ${ }^{11}$ D. Vucinic, ${ }^{16}$ R. G. Wagner, ${ }^{1}$ R. L. Wagner, ${ }^{7}$ J. Wahl, ${ }^{5}$ C. Wang, ${ }^{6}$ C. H. Wang, ${ }^{29}$ G. Wang, ${ }^{23}$ J. Wang, ${ }^{5}$ M. J. Wang, ${ }^{29}$ Q. F. Wang, ${ }^{27}$ A. Warburton, ${ }^{12}$ T. Watts, ${ }^{28}$ R. Webb, ${ }^{30}$ C. Wei, ${ }^{6}$ C. Wendt,${ }^{34}$ H. Wenzel, ${ }^{15}$ W. C. Wester, III, ${ }^{7}$
A. B. Wicklund, ${ }^{1}$ E. Wicklund, ${ }^{7}$ R. Wilkinson, ${ }^{22}$
H. H. Williams, ${ }^{22}$ P. Wilson, ${ }^{5}$
B. L. Winer, ${ }^{26}$ D. Wolinski, ${ }^{17} \mathrm{~J}$. Wolinski, ${ }^{18} \mathrm{X}$. Wu,${ }^{23} \mathrm{~J}$. Wyss,${ }^{21}$ A. Yagil,${ }^{7} \mathrm{~W}$. Yao, ${ }^{15}$
K. Yasuoka, ${ }^{32}$ Y. Ye ${ }^{12}$ G. P. Yeh, ${ }^{7}$ P. Yeh,${ }^{29}$ M. Yin,${ }^{6}$ J. Yoh, ${ }^{7}$ C. Yosef,${ }^{18}$ T. Yoshida,${ }^{20}$
D. Yovanovitch, ${ }^{7}$ I. Yu ${ }^{35}$ L. Yu, ${ }^{19}$ J. C. Yun, ${ }^{7}$ A. Zanetti, ${ }^{23}$ F. Zetti, ${ }^{23}$ L. Zhang, ${ }^{34}$ W. Zhang, ${ }^{22}$ and S. Zucchelli ${ }^{2}$

\section{(CDF Collaboration)}

1 Argonne National Laboratory, Argonne, Illinois 60439

2 Istituto Nazionale di Fisica Nucleare, University of Bologna, I-40126 Bologna, Italy

3 Brandeis University, Waltham, Massachusetts 02254

4 University of California at Los Angeles, Los Angeles, California 90024

5 University of Chicago, Chicago, Illinois 60637

6 Duke University, Durham, North Carolina 27708

7 Fermi National Accelerator Laboratory, Batavia, Illinois 60510

8 Laboratori Nazionali di Frascati, Istituto Nazionale di Fisica Nucleare, I-00044 Frascati, Italy

9 Harvard University, Cambridge, Massachusetts 02138

10 Hiroshima University, Higashi-Hiroshima 724, Japan

11 University of Illinois, Urbana, Illinois 61801

12 Institute of Particle Physics, McGill University, Montreal H3A 2T8, and University of Toronto,

Toronto M5S 1A7, Canada

13 The Johns Hopkins University, Baltimore, Maryland 21218

14 National Laboratory for High Energy Physics (KEK), Tsukuba, Ibaraki 305, Japan

15 Ernest Orland Lawrence Berkeley Laboratory, Berkeley, California 94720

16 Massachusetts Institute of Technology, Cambridge, Massachusetts 02139

17 University of Michigan, Ann Arbor, Michigan 48109

18 Michigan State University, East Lansing, Michigan 48824

19 University of New Mexico, Albuquerque, New Mexicu 87131 
21 Universita di Padova, Istituto Nazionale di Fisica Nucleare, Sezione di Padova, I-35191 Padova, Italy

22 University of Pennsylvania, Philadelphia, Pennsylvania 19104

23 Istituto Nazionale di Fisica Nucleare, University and Scuola Normale Superiore of Pisa, I-56100 Pisa, Italy 24 University of Pittsburgh, Pittsburgh, Pennsylvania 15260

25 Purdue University, West Lafayette, Indiana 47907

26 University of Rochester, Rochester, New York 14627

27 Rockefeller University, New York, New York 10021

28 Rutgers University, Piscataway, New Jersey 08854

29 Academia Sinica, Taipei, Taiwan 11529, Republic of China 30

Texas ABM University, College Station, Texas 77843

31 Texas Tech University, Lubbock, Texas 79409

32 University of Tsukuba, Tsukuba, Ibaraki 305, Japan

33 Tufts University, Medford, Massachusetts 02155

34 University of Wisconsin, Madison, Wisconsin 53706

35 Yale University, New Haven, Connecticut 06511 


\section{Bibliography}

[1] F. Wilczek, Ann. Rev. Nucl. Sci. 32, 177 (1982).

[2] S. L. Glashow, Nucl. Phys. 22, 579 (1961).

[3] S. Weinberg, Phys. Rev. Lett. 19, 1264 (1967).

[4] A. Salam, in Elementary Particle Theory, edited by N. Svartholm (Almqvist and Wiksell, Stockholm, 1968), p. 367.

[5] M. Gell-Mann, Phys. Lett. 8, 214 (1964).

[6] G. Zweig, CERN Reports 8182/TH.401, 1964, and CERN Reports 8419/TH.412, 1964; reprinted in Developments in the Quark Theory of Hadrons, edited by D. B. Lichtenberg and S. P. Rosen (Hadronic Press, Massachusetts, 1980).

[7] L. Montanet et al. (Particle Data Group), Phys. Rev. D 50, 1173 (1994).

[8] D. H. Perkins, Introduction to High Energy Physics (Addison-Wesley, California, 1987).

[9] D. Buskulic et al. (ALEPH Collaboration), Phys. Lett. B 349, 585 (1995).

[10] F. Abe et al. (CDF Collaboration), Phys. Rev. Lett. 74, 2626 (1995).

[11] P. Nason, S. Dawson and R. K. Ellis, Nucl. Phys. B303, 607 (1988).

[12] P. Nason, S. Dawson and R. K. Ellis, Nucl. Phys. B327, 49 (1989).

[13] N. Ellis and A. Kernan, Phys. Rep. 195, 23 (1990).

[14] A. D. Martin, W. J. Stirling and R. G. Roberts, Phys. Rev. D 47, 867 (1993).

[15] F. Abe et al. (CDF Collaboration), Phys. Rev. Lett. 75, 1451 (1995). 
[16] M. L. Mangano, P. Nason and G. Ridolfi, Nucl. Phys. B373, 295 (1992).

[17] C. Peterson, D. Schlatter, I. Schmitt and P. M. Zerwas, Phys. Rev. D 27, 105 (1983).

[18] J. Chrin, Z. Phys. C 36, 163 (1987).

[19] A. S. Schwarz, Phys. Rep. 238, 1 (1994).

[20] R. D. Field and R. P. Feynman, Nucl. Phys. B136, 1 (1978).

[21] V. Barger and R. Phillips, Collider Physics (Addison-Wesley, California, 1987).

[22] S. J. Brodsky and C. Peterson, in Proceedings of Europhysics Study Conference on High-Energy Physics, edited by G. Bellini and S. C. C. Ting (Plenum Press, New York, 1984), p. 363.

[23] J. D. Bjorken and G. R. Farrar, Phys. Rev. D 9, 1449 (1974).

[24] V. V. Anisovich and V. M. Shekhter, Nucl. Phys. B55, 455 (1973).

[25] V. V. Anisovich and M. N. Kobrinsky, Phys. Lett. 52B, 217 (1974).

[26] K. Cheung and R. J. Oakes, Phys. Lett. B 337, 181 (1994).

[27] F. Abe et al. (CDF Collaboration), Phys. Rev. Lett. 69, 3704 (1992).

[28] F. Abe et al. (CDF Collaboration), Phys. Rev. Lett. 75, 4358 (1995).

[29] K. Cheung and R. J. Oakes, Phys. Rev. D 53, 1242 (1996).

[30] P. Abreu et al. (DELPHI Collaboration), Phys. Lett. B 345, 598 (1995).

[31] D. Buskulic et al. (ALEPH Collaboration), Z Phys. C 69, 393 (1996).

[32] N. Cabibbo, Phys. Rev. Lett. 10, 531 (1963).

[33] M. Kobayashi and T. Maskawa, Prog. Theor. Phys. 49, 652 (1973).

[34] F. J. Gilman and M. B. Wise, Phys. Rev. D 20, 2392 (1979).

[35] M. Wirbel, Universität Dortmund Preprint, D0-TH 89/5, May 1989 (unpublished). 
[36] M. Wirbel, B. Stech and M. Bauer, Z. Phys. C 29, 637 (1985).

[37] M. Bauer, B. Stech and M. Wirbel, Z. Phys. C 34, 103 (1987).

[38] A. Deandrea, N. Di Bartolomeo, R. Gatto and G. Nardulli, Phys. Lett. B 318, 549 (1993).

[39] A. Deandrea, N. Di Bartolomeo, R. Gatto, F. Feruglio and G. Nardulli, Phys. Lett. B 320, 170 (1994).

[40] G. Kramer and W. F. Palmer, Phys. Rev. D 46, 2969 (1992).

[41] G. Kramer and W. F. Palmer, Phys. Rev. D 46, 3197 (1992).

[42] N. M. Gelfand, in Proceedings of the Symposium on High-Brightness Beams for Advanced Accelerator Applications, edited by W. W. Destler and S. K. Guharay (American Institute of Physics, New York, 1992), p. 111.

[43] E. McCrory, in Proceedings of the Fourth Annual Workshop on Accelerator Instrumentation, edited by J. A. Hinkson and G. Stover (American Institute of Physics, New York, 1993), p. 131.

[44] J. R. Sanford, Ann. Rev. Nucl. Sci. 26, 151 (1976).

[45] J. Peoples, in Physics of Particle Accelerators, Proceedings of the 1987 Fermilab Summer School and the 1988 Cornell Summer School, edited by M. Month and M. Dienes (American Institute of Physics, New York, 1989), p. 1845.

[46] D. Möhl, G. Petrucci, L. Thorndahl and S. van der Meer, Phys. Rep. 58, 73 (1980).

[47] D. A. Edwards, in Physics of Particle Accelerators, Proceedings of the 1987 Fermilab Summer School and the 1988 Cornell Summer School, edited by M. Month and M. Dienes (American Institute of Physics, New York, 1989), p. 1830.

[48] F. Abe et al., Nucl. Instr. Meth. Phys. A271, 387 (1988).

[49] S. Tkaczyk et al., Nucl. Instr. Meth. in Phys. Res. A 342, 240 (1994).

[50] D. Amidei et al., Nucl. Instr. Meth. in Phys. Res. A 350, 73 (1994). 
[51] F. Abe et al. (CDF Collaboration), Phys. Rev. D 52, 4784 (1995).

[52] F. Abe et al. (CDF Collaboration), Phys. Rev. D 50, 5550 (1994).

[53] F. Fames, CERN Program Library Long Writeup D506, Feynman Computing Number PM0020.

[54] F. Abe et al. (CDF Collaboration), Phys. Rev. D 50, 2966 (1994).

[55] F. DeJongh and R. Hans Level 1 and Level 2 Low $P_{t}$ Central Muon Trigger Efficiencies for Run IA, CDF Internal Note 1999, March 1995 (unpublished).

[56] F. Paige and S. D. Protopopecu, BNL Report No. 38034, 1986 (unpublished).

[57] P. Avery, K. Read and G. Trahern, Cornell Internal Note CSN-212, 1985 (unpublished).

[58] F. Abe et al. (CDF Collaboration), Phys. Rev. Lett. 75, 3068 (1995).

[59] T. Sjöstrand, Comput. Phys. Commun. 82, 74 (1994), and long writeup in Report No. CERN-TH.7112/93, August 1994 (unpublished).

[60] P. Abreu et al. (DELPHI Collaboration), Z Phys. C 68, 353 (1995).

[61] M. Acciarri et al. (L3 Collaboration), Phys. Lett. B 345, 589 (1995).

[62] D. Bortoletto et al., Phys. Rev. D 37, 1719 (1988).

[63] V. Ammosov et al., Phys. Lett. 93B, 210 (1980).

[64] P. K. Malhotra and R. Orava, Z. Phys. C 17, 85 (1983).

[65] W. Bartel et al. (JADE Collaboration), Z. Phys. C 20, 187 (1983).

[66] H. Aihara et al., Phys. Rev. Lett. 53, 2378 (1984).

[67] G. J. Alner et al. (UA5 Collaboration), Nucl. Phys. B258, 505 (1985).

[68] R. E. Ansorge et al. (UA5 Collaboration), Z. Phys. C 41, 179 (1988).

[69] F. Abe et al., Phys. Rev. D 40, 3791 (1989).

[70] P. Abreu et al. (DELPHI Collaboration), Z. Phys. C 61, 407 (1994). 
[71] D. Buskulic et al. (ALEPH Collaboration), Z. Phys. C 69, 379 (1996).

[72] G. Bocquet et al., Phys. Lett. B 366, 447 (1996).

[73] M.S. Alam et al. (CLEO Collaboration), Phys Rev. D 50, 43 (1994).

[74] H. Albrecht et al. (ARGUS Collaboration), Phys. Lett. B 340, 217 (1994).

[75] M. Gourdin, A. N. Kamal and X. Y. Pham, Phys. Rev. Lett. 73, 3355 (1994).

[76] H. Albrecht et al. (ARGUS Collaboration), Z. Phys. C 48, 543 (1990).

[77] K. Cheung and T. C. Yuan, Phys. Lett. B 325, 481 (1994).

[78] K. Cheung and T. C. Yuan, Phys. Rev. D 53, 1232 (1996).

[79] P. Abreu et al. (DELPHI Collaboration), Z. Phys. C 68, 375 (1995).

[80] D. Buskulic et al. (ALEPH Collaboration), Phys. Lett. B 357, 685 (1995).

[81] R. Akers et al. (OPAL Collaboration), Z. Phys. C 69, 195 (1996).

[82] T. Mannel and G. A. Schuler, Phys. Lett. B 279, 194 (1992).

[83] S. Kim and J. Suzuki Update on the search for $B_{c}^{+} \rightarrow e^{+} J / \psi X$ using Run Ia and Run Ib data, CDF Internal Note 3287, April 1996 (unpublished).

[84] F. Abe et al. (CDF Collaboration), Phys. Rev. Lett. 67, 3351 (1991). 